\title{
Photodissociation and photoionisation of atoms and molecules of astrophysical interest
}

\author{
A. N. Heays ${ }^{\star}$, A. D. Bosman, and E. F. van Dishoeck
}

Leiden Observatory, Leiden University, PO Box 9513, 2300 RA Leiden, The Netherlands
e-mail: heays@strw. leidenuniv.nl

Received 19 April 2016 / Accepted 1 January 2017

\begin{abstract}
A new collection of photodissociation and photoionisation cross sections for 102 atoms and molecules of astrochemical interest has been assembled, along with a brief review of the basic physical processes involved. These have been used to calculate dissociation and ionisation rates, with uncertainties, in a standard ultraviolet interstellar radiation field (ISRF) and for other wavelength-dependent radiation fields, including cool stellar and solar radiation, Lyman- $\alpha$ dominated radiation, and a cosmic-ray induced ultraviolet flux. The new ISRF rates generally agree within $30 \%$ with our previous compilations, with a few notable exceptions. Comparison with other databases such as PHIDRATES is made. The reduction of rates in shielded regions was calculated as a function of dust, molecular and atomic hydrogen, atomic $\mathrm{C}$, and self-shielding column densities. The relative importance of these shielding types depends on the atom or molecule in question and the assumed dust optical properties. All of the new data are publicly available from the Leiden photodissociation and ionisation database. Sensitivity of the calculated rates to variation of temperature and isotope, and uncertainties in measured or calculated cross sections, are tested and discussed. Tests were conducted on the new rates with an interstellar-cloud chemical model, and find general agreement (within a factor of two) in abundances obtained with the previous iteration of the Leiden database assuming an ISRF, and order-of-magnitude variations assuming various kinds of stellar radiation. The newly parameterised dust-shielding factors makes a factor-of-two difference to many atomic and molecular abundances relative to parameters currently in the UDfA and KIDA astrochemical reaction databases. The newly-calculated cosmic-ray induced photodissociation and ionisation rates differ from current standard values up to a factor of 5. Under high temperature and cosmic-ray-flux conditions the new rates alter the equilibrium abundances of abundant dark cloud abundances by up to a factor of two. The partial cross sections for $\mathrm{H}_{2} \mathrm{O}$ and $\mathrm{NH}_{3}$ photodissociation forming $\mathrm{OH}, \mathrm{O}, \mathrm{NH}_{2}$ and $\mathrm{NH}$ are also evaluated and lead to radiation-field-dependent branching ratios.
\end{abstract}

Key words. photon-dominated region (PDR) - cosmic rays - dust, extinction - ISM: molecules - molecular data - atomic data

\section{Introduction}

Ultraviolet (UV) photons play a critical role in interstellar and circumstellar chemistry. The realisation that photodissociation and photoionisation processes control the abundances of atoms and small molecules in diffuse interstellar clouds dates back nearly a century (Eddington 1928; Kramers \& Ter Haar 1946; Bates \& Spitzer 1951). Similarly, photodissociation of parent species by UV radiation from the Sun has long been known to explain the existence of small molecules in cometary comae (Haser 1957; Crovisier et al. 1997). Nowadays, photodissociation processes are found to be important for modelling the chemistry of nearly every type of astrophysical region, from the edges of dense clouds close to bright young stars to the surface layers of protoplanetary disks, envelopes around evolved stars and giant molecular clouds on galactic scales (e.g., Glassgold 1996; Hollenbach \& Tielens 1999; Tielens 2013; van Dishoeck et al. 2006; Glover \& Clark 2012). Such clouds of gas and dust in which photodissociation is the dominant molecular destruction path are termed photodissociation or photon-dominated regions (PDRs), although the term PDRs originally referred mostly to high density regions close to bright $\mathrm{O}$ and $\mathrm{B}$ stars such as found in Orion (Tielens \& Hollenbach 1985).

* Current contact: Observatoire de Paris, LERMA, UMR 8112 du CNRS, 92195 Meudon, France.
The abundant UV photons in these regions photodissociate and photoionise the main hydrogen, carbon, oxygen and nitrogen-containing species, controlling the $\mathrm{H}^{+} \rightarrow \mathrm{H} \rightarrow$ $\mathrm{H}_{2}, \mathrm{C}^{+} \rightarrow \mathrm{C} \rightarrow \mathrm{CO}, \mathrm{O} \rightarrow \mathrm{O}_{2}$ and $\mathrm{N} \rightarrow \mathrm{N}_{2}$ transitions (Tielens \& Hollenbach 1985; van Dishoeck \& Black 1988; $\mathrm{Li}$ et al. 2013). Photoprocesses thus affect the abundance of the main cooling species in the interstellar medium, and they also generate chemically-reactive ions and radicals, opening pathways to the formation of larger species (Sternberg \& Dalgarno 1995; Lee et al. 1996; Jansen et al. 1996; Li et al. 2014). The gas-phase abundance of more complex molecules formed in this way is simultaneously limited by their own photodestruction (Teyssier et al. 2004; van Hemert \& van Dishoeck 2008; Guzman et al. 2014). The photoionisation of atoms and molecules also leads to a significant speed up of PDR chemistry due to the enhanced reaction rates of ions compared with neutral species (Tielens 2013; van Dishoeck 2014).

The quantitative modelling of chemical evolution in clouds, envelopes and disks is a prerequisite for the full interpretation of observations of their emitting molecular lines and dust continuum. Such models consider many physical regimes (e.g., Le Petit et al. 2006; Walsh et al. 2013) and involve many classes of chemical reactions (Wakelam et al. 2012; McElroy et al. 2013). By quantitatively constraining the rates of photoprocesses, as is done in this paper, other chemical and physical 
parameters processes affecting observations can be more reliably determined.

The fundamental quantities governing photodissociation and ionisation are the wavelength-dependent flux of incident UV radiation, discussed in Sect. 2, and the wavelength-dependent photoabsorption, photodissociation, and photoionisation cross sections of each atom or molecule, introduced in Sects. 3 and 4. Historically, the complete and unabridged specification of these quantities contained too much information to be included in astrochemical models, and is actually in many cases unnecessary given the scale of uncertainties in observations and other model parameters. Tabulated pre-integrations of the full wavelength dependence into a process rate (or lifetime) for different species in different kinds of UV-irradiated environments are useful to speed up modelling. We calculated such rates in Sect. 5. Such tabulations must necessarily include the column-density-dependent effect of radiation shielding by dust, $\mathrm{H}$ and $\mathrm{H}_{2}$ inside interstellar and circumstellar clouds. The wavelength dependence of such shielding is frequently presented by a simplified parameterisation and is discussed further in Sect. 6.

Astrochemical models can also use the full molecular and atomic cross sections as functions of wavelength, and consider the dissociation of species and shielding by $\mathrm{H}$ and $\mathrm{H}_{2}$ line-by-line, to compute the photodestruction of molecules as functions of depth into a PDR (e.g., van Dishoeck \& Black 1988; Viala et al. 1988; Jansen et al. 1995; Le Petit et al. 2006; Woitke et al. 2009; Walsh et al. 2013; Li et al. 2013). Furthermore, astrochemical programs that employ simplified rates for photodestruction may require precomputing many of these when exploring, for example, a range of possible dust grain ultraviolet extinction properties (e.g., van Dishoeck et al. 2006; Röllig et al. 2013). Fundamental atomic and molecular cross sections such as those presented here are then required.

Even deep inside dark clouds well shielded from external radiation, a weak UV flux is maintained. This is induced by the interaction of cosmic rays with hydrogen. The resulting spectrum is highly structured (Prasad \& Tarafdar 1983; Gredel et al. 1987) and incorporation of this process into astrochemical models also benefits from a reduction of the full wavelength dependence into a conveniently tabulated rate. The most-recent tabulation of these rates is by Gredel et al. (1989). Since that time there have been updates for many of the photodissociation cross sections of astrophysically relevant molecules. Here we update these rates in Sect. 7.

In Sect. 8, we discuss the potential variability of our collected cross sections and calculated rates given their dependence on: interstellar dust optical properties, temperature, spectrally unresolved cross sections, and isotopic substitution. We also make a special case of studying distinct fragment branching ratios from the photodissociation of $\mathrm{H}_{2} \mathrm{O}$ and $\mathrm{NH}_{3}$, and assess the significance of our new rates by means of a physically simple but chemically complex toy astrochemical model.

All cross sections and calculated rates are available from the Leiden Observatory database of "photodissociation and photoionisation of astrophysically relevant molecules"1, and any future updates will be available there. Some of these cross sections are carried over from the previous iteration of the Leiden database (van Dishoeck 1988; van Dishoeck et al. 2006); many species are updated where new experimental or theoretical data has become available, especially using the MPI Mainz $\mathrm{UV} / \mathrm{Vis}$ database ${ }^{2}$. The list of molecules in the database has been

\footnotetext{
1 http://www.strw. leidenuniv.nl/ ewine/photo

2 http://satellite.mpic.de/spectral_atlas
}

extended by new additions of complex-organic species that have recently been detected in the interstellar medium ${ }^{3}$.

\section{Radiation fields}

The photodissociation or photoionisation rate (molec. atom ${ }^{-1} \mathrm{~s}^{-1}$ ) of a molecule (or atom) exposed to an ultraviolet radiation field is

$k=\int \sigma(\lambda) I(\lambda) \mathrm{d} \lambda$

where $\sigma(\lambda)$ is the appropriate photodissociation or photoionisation cross section, to be discussed in Sect. 3, and $I(\lambda)$ is the photon-based radiation intensity summed over all incidence angles. A photon-counting intensity was used for calculations in this paper because of the discrete nature of photodestruction events, but is directly related to the volumetric radiation energy density according to $U(\lambda)=h I(\lambda) / \lambda$ where $h$ is the Planck's constant. An angularly-differential radiation intensity may be appropriate if the incident radiation is non-isotropic. The integration limits in Eq. (1) are defined by the wavelength range corresponding to the nonzero photodissociation or ionisation cross section and radiation intensity.

The average intensity of the interstellar radiation field (ISRF) can be estimated from the number and distribution of hot stars in the Galaxy, combined with a model for the dust distribution and its extinction of the stellar radiation (Habing 1968; Draine 1978; Mathis et al. 1983; Parravano et al. 2003). The various estimates of the mean UV energy density at a typical point in the local galaxy agree to within a factor of two. Variations in this energy density of a factor between two and three are expected throughout the galactic plane and on time scales of a few Gyr, as massive $\mathrm{O}$ and $\mathrm{B}$ star clusters form and die. In addition, the intensity ratio of short-wavelength photons capable of dissociating $\mathrm{H}_{2}, \mathrm{CO}$ and $\mathrm{N}_{2}$ and ionising atomic $\mathrm{C}(\lambda<110 \mathrm{~nm})$ and the broader far-ultraviolet range $(91.2<\lambda<200 \mathrm{~nm})$ may vary by a factor of two in location and time (Parravano et al. 2003).

The wavelength dependent UV intensity as defined by Draine (1978) is often adopted in astrochemical models, and given by the formula

$$
\begin{aligned}
I(\lambda)= & 3.2028 \times 10^{13} \lambda^{-3}-5.1542 \times 10^{15} \lambda^{-4} \\
& +2.0546 \times 10^{17} \lambda^{-5}
\end{aligned}
$$

where the wavelength, $\lambda$, has units of $\mathrm{nm}$ and the radiation intensity, $I$, has units of photons $\mathrm{cm}^{-2} \mathrm{~s}^{-1} \mathrm{~nm}^{-1}$. This formula was intended for application within the 91.2 to $200 \mathrm{~nm}$ wavelength range. An angularly-differential Draine field, $I(\lambda) / 4 \pi$, has units of photons $\mathrm{cm}^{-2} \mathrm{~s}^{-1} \mathrm{~nm}^{-1} \mathrm{sr}^{-1}$; and a scaled version of the radiation intensity may be adopted, $\chi I(\lambda)$, to describe regions with greater or lesser UV flux than the mean intensity defined by Draine.

The form of Eq. (2) is shown in Fig. 1 and is reminiscent of a $20000 \mathrm{~K}$ black-body radiation field (B-type star) with some excess at shorter wavelengths. There is assumed to be zero flux shortwards of $91.2 \mathrm{~nm}$ due to the ionisation continuum of atomic $\mathrm{H}$ that populates the interstellar medium with a high column density for all sight lines. An extension proposed by van Dishoeck \& Black (1982) simulates the interstellar flux at longer wavelengths than considered by the Draine model, and

\footnotetext{
3 A community supported list of interstellar molecules: http://wikipedia.org/wiki/List_of_interstellar_and_ circumstellar_molecules
} 


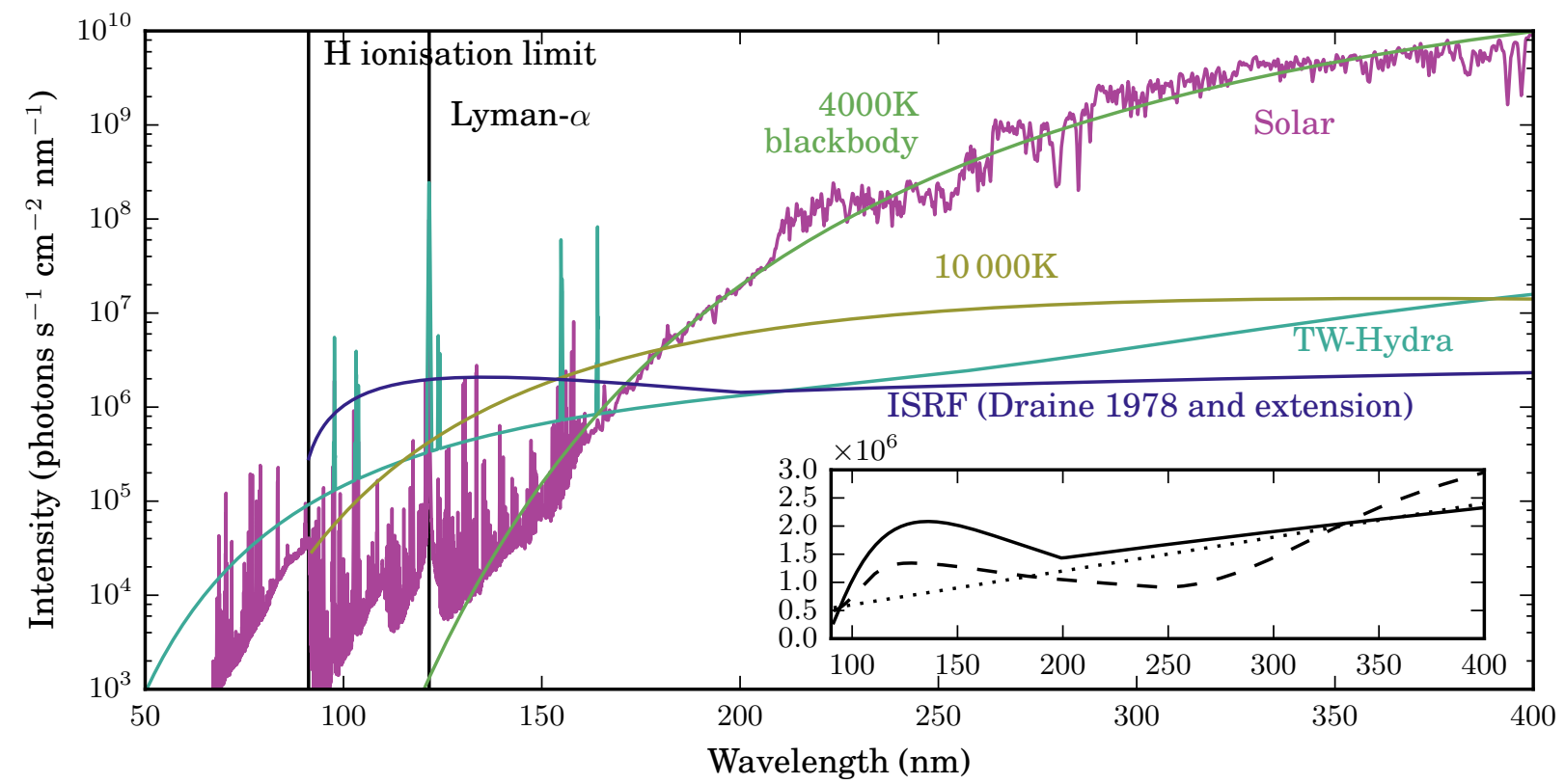

Fig. 1. Wavelength dependence of some astrophysically-relevant ultraviolet radiation fields. Inset: radiation intensity in the solar neighbourhood estimated by Draine (1978; solid, modified according to van Dishoeck \& Black 1982), Mathis et al. (1983; dashed), and Habing (1968; dotted).

fits a range of observed intensities between 200 and $2000 \mathrm{~nm}$ to within about $50 \%$. This extension is given by the formula:

$I(\lambda)=3.67 \times 10^{4} \lambda^{0.7} ; \lambda>200 \mathrm{~nm}$.

We combine the full wavelength range of the Draine (1978) and van Dishoeck \& Black (1982) fields into a "standard" ISRF for the following calculations of photodissociation and ionisation rates.

The energy intensity of the Draine field integrated between 91.2 and $200 \mathrm{~nm}$ is,

$\int_{91.2}^{200} \frac{h c I(\lambda)}{\lambda} \mathrm{d} \lambda=2.6 \times 10^{-6} \mathrm{~W} \mathrm{~m}^{-2}$,

where $h$ is the Planck's constant, and $c$ the speed of light. This integrated value is a factor of 1.7 higher than the integrated flux of the Habing (1968) field, which is taken as the reference with scaling factor $G_{0}$ in some models (Tielens \& Hollenbach 1985). Thus, the standard Draine field has $G_{0}=1.7$.

An independent estimate of the Galactic radiation field is made by Mathis et al. (1983), and its magnitude and wavelength dependence for the case of $10 \mathrm{kpc}$ Galactocentric distance (the local Galaxy) is compared in Fig. 1 with the ISRF standard we adopted. The Mathis et al. UV flux is generally about $35 \%$ weaker, and photodissociation rates will be similarly reduced for all atoms and molecules, apart from those that are photodestroyed at wavelengths longer than $300 \mathrm{~nm}$, at which point the Mathis radiation becomes stronger than our standard ISRF.

The ultraviolet field near to a star is dominated by its black body radiation and atomic emission or absorption lines, principally the HI Lyman- $\alpha$ emission line at $121.6 \mathrm{~nm}$. We model several such radiation fields as pure black-body emitters in the following calculations. Special attention to the Lyman- $\alpha$ emission spectrum is warranted because of the known high intensity of this feature in some astrophysical situations, including fast shocks (Neufeld \& Dalgarno 1989), the active Sun (Lammer et al. 2012), and young stars (Valenti et al. 2000; Yang et al. 2012). Indeed, around some T Tauri stars, up to $90 \%$ of the total far-ultraviolet flux is emitted in the Lyman- $\alpha$ band (Bergin et al. 2003; Schindhelm et al. 2012). Also, the propagation of Lyman- $\alpha$ radiation into a disk is significantly enhanced by scattering from the disk surface (Bethell \& Bergin 2011), where a $121.6 \mathrm{~nm}$ photon absorbed by an $\mathrm{H}$ atom will be ultimately reradiated in a random direction, including into the disk. Thus, we also treat a pure Lyman- $\alpha$ line in our calculations. A $200 \mathrm{~km} \mathrm{~s}^{-1}$ Doppler broadening is added to the Lorentzian natural linewidth of the Lyman- $\alpha$ transition. This broadening is a typical value from the observationally-constrained photospheric emission of a sample of T-Tauri stars (France et al. 2014).

In reality, stellar spectra are not black bodies but contain many emission or absorption lines (e.g., Ardila et al. 2002a,b; Leitherer et al. 2010). As an example of a structured stellar flux, we consider a combination of continuum and atomic emission simulating the photosphere of the classical T-Tauri star TW-Hydra, as deduced from UV telescope observations (France et al. 2014). This observationally-derived spectrum is extrapolated to shorter and longer wavelengths using the derived black body and accretion-induced short wavelength excess, respectively, proposed by Nomura \& Millar (2005). This includes an additional nonblack-body ultraviolet excess due to the accretion of material onto the still-forming star.

The solar ultraviolet flux is measured directly in the series of SOHO-SUMER observations (Curdt et al. 2001) for $\lambda<160 \mathrm{~nm}$ and also by the UARS SOLSTICE mission (Woods et al. 1996), including longer wavelengths. We adopt a spectrum compiled from these two data sets corresponding to a quiet period in the Sun's radiance. The activity level of the Sun makes little difference for $\lambda>160 \mathrm{~nm}$ but can induce variation of a factor of two or more at shorter wavelengths, including enhanced Lyman- $\alpha$ radiation. More detailed studies of the dependence of molecular photodissociation rates on solar activity are made by Huebner et al. (1992) and Huebner \& Mukherjee (2015).

All stellar radiation fields were normalised to match the energy intensity of the Draine field integrated between 91.2 and $200 \mathrm{~nm}$, that is, $2.6 \times 10^{-6} \mathrm{~W} \mathrm{~m}^{-2}$. The photodissociation 
and ionisation rates calculated hereafter due to exposure of molecules and atoms to these radiation field should subsequently be scaled to match the flux in an astrophysical environment, which may differ by multiple orders of magnitude. Our normalisation scheme is selected to emphasise the wavelengthdependent effects induced by substituting radiation fields. A scale factor of 37700 should be used to increase the solar photodissociation and photoionisation rates calculated here to values appropriate for the approximate solar intensity at $1 \mathrm{au}$, assuming an integrated solar flux between 91.2 and $200 \mathrm{~nm}$ of $0.098 \mathrm{~W} \mathrm{~m}^{-2}$.

For the cases of the solar and TW-Hydra radiation fields, intensity at wavelengths shorter than the ionisation threshold of atomic $\mathrm{H}, 91.2 \mathrm{~nm}$, is included. This is certainly appropriate for studies of planetary atmospheres and cometary comae in the Hdeprived solar system. There are also several known cases of highly-evolved, hydrogen gas-poor debris disks supporting some amount of CO (Mathews et al. 2014). The origin of this gas is unknown but may arise from evaporation of solids in collisions of planetesimals, allowing for relatively low amounts of gasphase hydrogen relative to other species and the free transmission of short-wavelength radiation (Dent et al. 2014).

A cosmic-ray induced UV-emission spectrum is taken from the calculations of Gredel et al. (1989). The energetic electrons produced from cosmic-ray ionisation of hydrogen excite $\mathrm{H}_{2}$ into excited electronic states. Spontaneous emission back to the electronic ground state produces a rich spectrum of UV lines, from 91.2 to $170 \mathrm{~nm}$, as well as a weak continuum between 150 and $170 \mathrm{~nm}$. The precise spectral details depend on the initial population of $\mathrm{H}_{2}$ ro-vibrational levels and the ortho-to-para ratio of $\mathrm{H}_{2}$. Usually $\mathrm{H}_{2}$ is assumed to be in its vibrational and rotational ground state in the cold interiors of dark clouds.

\section{Cross sections}

\subsection{General properties}

The critical data needed to describe gas-phase molecular or atomic photoprocesses is the wavelength-dependent photoabsorption cross section, $\sigma(\lambda)$. This differential quantity describes the expected rate of photoabsorption per spectral unit of an isolated molecule or atom, $\mathrm{ABC}$, in a photon-intensity normalised radiation field, bringing it into an excited electronic state $\mathrm{ABC}^{*}$, and (oddly) has dimension of area. The optical depth of the absorption at a certain wavelength is given by $\tau=N \times \sigma$. Thus, a cloud of molecules with cross section $\sigma(\lambda)=10^{-17} \mathrm{~cm}^{2}$ and column density $N=10^{17} \mathrm{~cm}^{-2}$ has an optical depth of 1 , and a $1 / e$ probability of absorbing a photon with wavelength $\lambda$.

A photo-excited molecule $\mathrm{ABC}^{*}$ may decay by several channels, and the probability of each of them needs to be taken into account. This includes dissociation (e.g., forming $\mathrm{A}+\mathrm{BC}$ ), ionisation $\left(\mathrm{ABC}^{+}+\mathrm{e}^{-}\right)$, or non-destructive emission $(\mathrm{ABC}+$ photon). Their respective partial photodissociation, photoionisation, and photoemission cross sections $\sigma^{\mathrm{d}}(\lambda), \sigma^{\mathrm{i}}(\lambda)$, and $\sigma^{\mathrm{e}}(\lambda)$, are the product of the photoabsorption cross section and a decay probability, $\eta^{\mathrm{d}}(\lambda), \eta^{\mathrm{i}}(\lambda)$, and $\eta^{\mathrm{e}}(\lambda)$; respectively. We generally neglected further division of the photoabsorption cross section into decay channels leading to distinct dissociation products (e.g., $\mathrm{A}+\mathrm{BC}, \mathrm{AB}+\mathrm{C}$, or $\mathrm{A}+\mathrm{B}+\mathrm{C}$ ) or dissociativeionisation fragments (e.g., $\mathrm{ABC}^{+}$or $\mathrm{AB}^{+}+\mathrm{C}$ ) because of limited branching-ratio data in the literature, although this is a very relevant issue for chemical models. In general, multiple fragments are energetically possible and participate distinctly in ongoing chemistry, for example, $\mathrm{CH}_{4}$ dissociating to form significant amounts of $\mathrm{CH}_{3}$ and $\mathrm{CH}_{2}$ in Titan's atmosphere (Romanzin et al. 2005), or $\mathrm{H}_{2} \mathrm{O}$ dissociating into $\mathrm{OH}+\mathrm{H}$ or $\mathrm{O}+\mathrm{H}_{2}$, with a wavelength-dependent relative likelihood. As an exception, in Sect. 8.6 we undertake to characterise the photodissociation branching of $\mathrm{H}_{2} \mathrm{O}$ into $\mathrm{OH}$ and $\mathrm{H}$ products, and $\mathrm{NH}_{3}$ into $\mathrm{NH}_{2}$ and $\mathrm{NH}$.

The wavelength dependence of a molecular cross section can be schematically associated with the structure of its electronically-excited states and categorised by its dissociation mechanism. These mechanisms are depicted in Fig. 2 by potential-energy curves. For small molecules absorption into an excited state whose potential is repulsive along 1 or more nuclear coordinates results in $100 \%$-efficient direct dissociation of the molecule on sub-picosecond time scales (see Sect. 3.3 and Fig. 3 for a description and example of potential energy curves). The corresponding cross section has a broad wavelength distribution, covering several $\mathrm{nm}$ decades and peaking at the energy corresponding to vertical excitation from the ground-state equilibrium nuclear distance to the excited repulsive curve of $\mathrm{AB}^{*}$. Typical peak values range from a few $\times 10^{-18}$ to a few $\times 10^{-17} \mathrm{~cm}^{2}$.

In contrast, the cross sections for the predissociation and indirect predissociation processes are highly structured, consisting of sharp peaks at discrete wavelengths. In these cases, the initial absorption occurs into a bound excited electronic state, which subsequently interacts non-radiatively with a nearby repulsive electronic state. The predissociation rate, and inverselyproportional linewidth, depends strongly on the details of this interaction and may vary from level to level, particularly in the indirect case where further intermediate states are involved. A non-unity dissociation probability will result from competitive rates for predissociation, $k^{\text {pre }}$, and spontaneous emission, $A$; so that $\eta^{\mathrm{d}}=k^{\text {pre }} /\left(k^{\text {pre }}+A\right)$. An excited molecule decaying by emission may follow multiple competing pathways involving multiple photons of different wavelengths in a de-excitation cascade through excited and ground electronic states, and result in a super-thermal population of ground state rotational and vibrational levels. Only the total emissive decay rate, $A$, is considered in this paper. $\mathrm{CO}$ and $\mathrm{N}_{2}$ are the best known astrophysical examples of molecules for which predissociation is dominant.

The fourth process is spontaneous radiative dissociation, in that an excited bound state radiates back into the vibrational continuum of a lower state with a line-dependent probability. For $\mathrm{H}_{2}$, this is the dominant photodissociation pathway (Stecher \& Williams 1967), but not for any other interstellar molecule. Peak cross sections for discrete lines may reach $10^{-14} \mathrm{~cm}^{2}$ over a width of $<0.1 \mathrm{~nm}$.

Even though the peak cross sections may differ greatly for the various dissociation mechanisms depicted in Fig. 2, the integrated cross sections $\int \sigma(\lambda) \mathrm{d} \lambda$ are often comparable. Further discussion and details of these phenomena may be found in van Dishoeck (1988) and van Dishoeck \& Visser (2015).

As a real example, Fig. 3 illustrates that both direct continuous and discrete dissociation channels are available for a molecule like $\mathrm{O}_{2}$. The appearance of continuum absorption between 180 and $130 \mathrm{~nm}$ in Fig. 3 is consistent with an upward projection of the ground-state vibrational wavefunction to its intersection with the lowest-energy unbound excited state. The line absorption at shorter wavelengths occurs through the predissociation of multiple bound states above $9.2 \mathrm{eV}$.

Large molecules such as polycyclic aromatic hydrocarbons (PAHs) are much more stable against photodissociation than the small species considered here because the absorptions are followed by non-radiative decay to the ground state (so-called internal conversion) from which there is only a small probability 
A. N. Heays et al.: Photodissociation and photoionisation of atoms and molecules of astrophysical interest

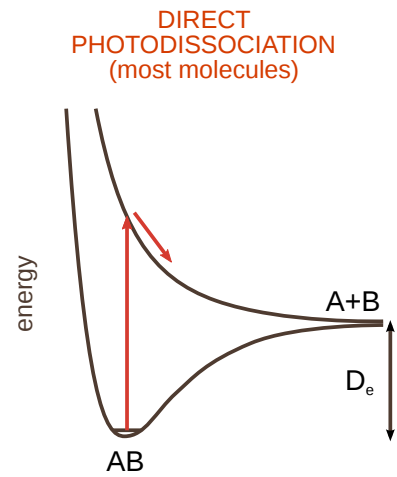

internuclear distance

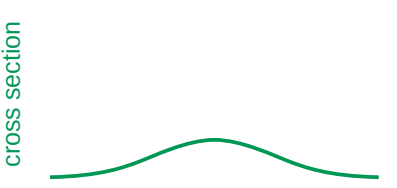

photon frequency
PREDISSOCATION $\left(\mathrm{NH}_{3}\right)$

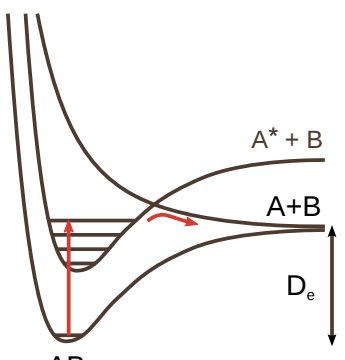

$\mathrm{AB}$

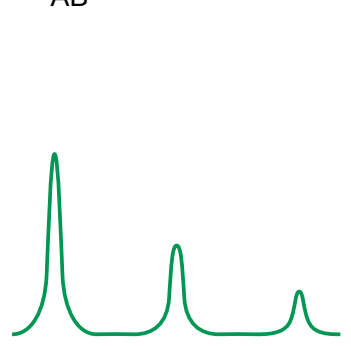

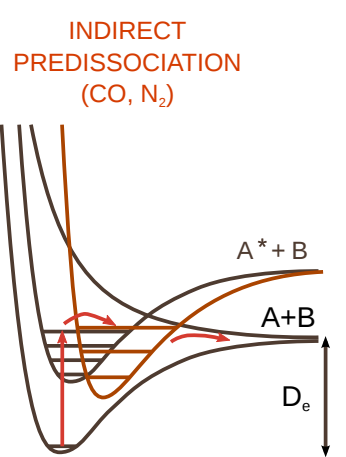

$A B$

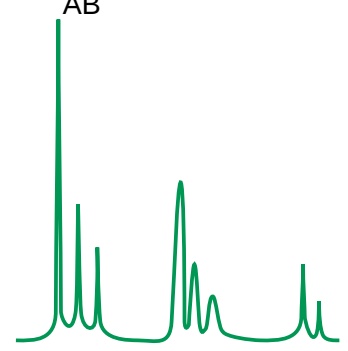

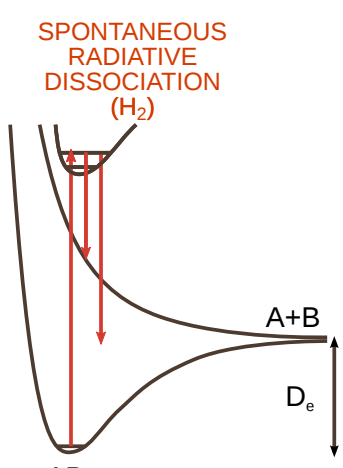

$\mathrm{AB}$

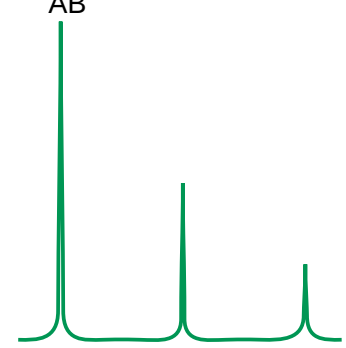

Fig. 2. Schematic cross sections for photodissociation and their associated dynamical pathway (arrows) through ground and excited state potentialenergy curves. For polyatomic molecules these curves represent a cross section through a multidimensional energy surface. We note that the integrated values of the various cross sections may be similar, leading to orders-or-magnitude greater peak magnitudes for indirect mechanisms. Modified from van Dishoeck \& Visser (2015).

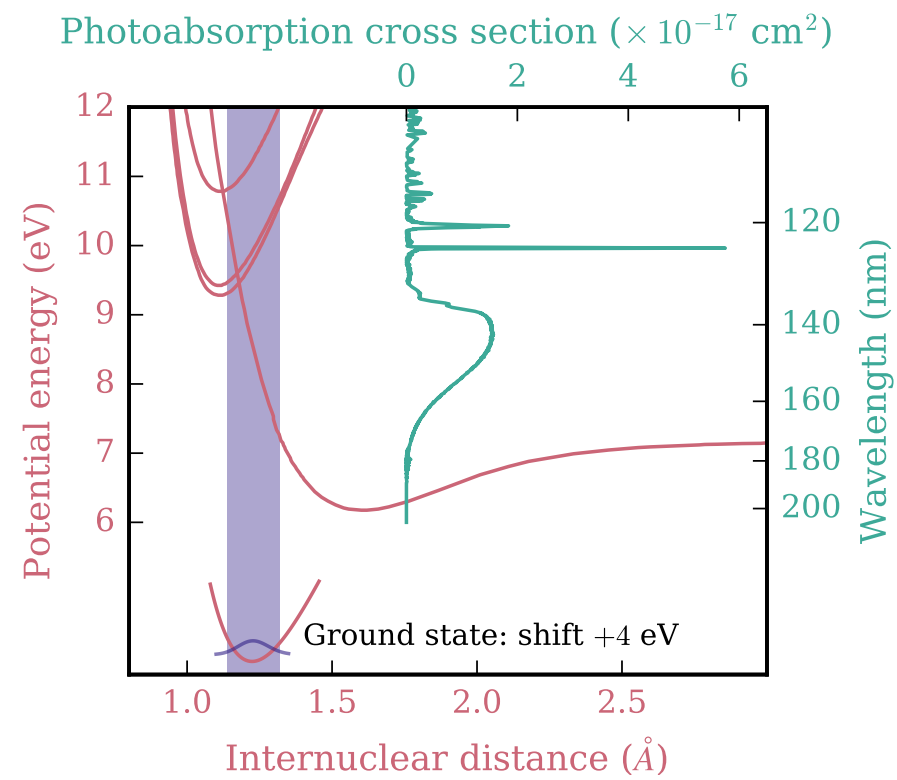

Fig. 3. Potential energy curves for the ground and excited state of $\mathrm{O}_{2}$ (red curves) (Guberman 1977; Lewis et al. 1998, 2001), and the $\mathrm{O}_{2}$ photoabsorption section from Sect. 4.3 .35 (blue curve). Shown on equivalent energy and wavelength scales. The energy scale is relative to the minimum of the ground-state electronic potential curve, shown here shifted upwards by $4 \mathrm{eV}$. The shaded area shows the vertical excitation region.

that the molecule finds a path to dissociation. In clouds exposed to very intense UV radiation, such as protoplanetary disks or near active galactic nuclei, photodissociation may however become significant on astronomical time scales, and small PAHs (less than about 50 carbon atoms) cannot survive.
Photodissociation of these large molecules was not taken into account for this database but is discussed most recently by Le Page et al. (2003) and Visser et al. (2007; see also summary in van Dishoeck \& Visser 2015). New experimental data on the photofragmentation and ionisation probabilities of PAHs is becoming available (Zhen et al. 2015, 2016).

In general, the key characteristics of a photoabsorption cross section are:

- The long-wavelength dissociation threshold: usually this is given by the dissociation energy of the ground electronic state. The cross section at this threshold is often orders of magnitude smaller than at shorter wavelengths. However, radiation intensity decreases rapidly with shortening wavelength for radiation fields dominated by cool stars, so even a low cross section near threshold can dominate the photodissociation rate.

- The ionisation threshold: this affects the relative importance of photodissociation and photoionisation. No ionisation will occur in most astrophysical environments if this threshold occurs at wavelengths shorter than $91.2 \mathrm{~nm}$.

- The wavelengths of absorption lines: maxima in the cross section can influence the total absorption rate if they correspond to emission lines, such as occur in the simulated TWHydra radiation field, or in cosmic-ray induced radiation.

- The cross section corresponding to the Lyman- $\alpha$ emission line at $121.3 \mathrm{~nm}$, that can singularly dominate the ultraviolet flux.

- The characteristic width of absorption features: the precise linewidths, that can range from 0.001 to several $10 \mathrm{~s}$ of $\mathrm{nm}$ has a strong effect on their ability to self-shield (Sect. 6).

Further background information and descriptions of the collected data sources and cross sections for all updated and new species relative to van Dishoeck et al. (2006) are given in Sect. 4. 
In the following subsections, more background information on experimental and theoretical determinations of cross sections is given, since this is relevant for assessing the inherent uncertainty of the various data we used, and motivating our choices of adopted cross sections.

\subsection{Experimental cross sections}

Photoabsorption cross sections are most frequently recorded directly, by observing the transmission of an ultraviolet continuum through a gas sample, with radiation generated by discharge lamps (e.g., Ogawa \& Ogawa 1975; Dehmer \& Chupka 1976) or synchrotrons (e.g., Yoshino et al. 1996; Cheng et al. 2011), and dispersed by diffraction gratings or interferometry (Yoshino et al. 2006). Laser-generated ultraviolet radiation is sometimes used in photoabsorption experiments and provides the highest spectral resolution (Gao et al. 2013; Niu et al. 2014), but generally cannot be tuned over a large wavelength range or provide controllable intensity. Special techniques are required to record ultraviolet photoabsorption spectra for wavelengths shorter than $105 \mathrm{~nm}$ due to the lack of transmitting material for use as windows or beam splitters. For example, utilising frequency-multiplied lasers (e.g., Ubachs 2005; Stark et al. 1999), synchrotron radiation sources (e.g., Yoshino et al. 2006) and, recently, the vacuum-ultraviolet Fourier transform spectrometer at the SOLEIL synchrotron (de Oliveira et al. 2011; Eidelsberg et al. 2012), or occasionally the interstellar laboratory (e.g., Federman et al. 2001).

The interpretation of experimental photoabsorption spectra is generally straight-forward except where the instrumental spectral resolution is insufficient to resolve detailed structure of molecules with non-continuum absorption (cf., $\mathrm{N}_{2}$ as opposed to $\mathrm{CH}_{4}$ in Sect. 4). In this case, care needs to be taken not to underestimate the integrated cross section, potentially by more than an order of magnitude (Hudson \& Carter 1968). Due to this issue, for some molecules (e.g., $\mathrm{H}_{2}, \mathrm{~N}_{2}$, and $\mathrm{CO}$ ) the recorded absorption spectra must be analysed line-by-line and the true cross section reconstructed without the limitation of experimental broadening (Eidelsberg et al. 1992; Heays et al. 2011; Glass-Maujean et al. 2013c).

Another difficulty concerns the calibration of absolute cross section values, which rely on precise knowledge of the absorbing sample gas column density and distribution of the ground-state rovibrational population. Neither quantity is generally diagnosable in photoabsorption experiments involving transient radical species. The uncertainty of directly-measured photoabsorption cross sections is usually between 10 to $20 \%$ for stable species, and typically a factor of 2 to 5 for the case of radicals, if it is known at all.

A photoabsorption cross section can also be estimated at relatively-low resolution by electron-energy-loss spectroscopy (e.g., Chan et al. 1992; Heays et al. 2012), where monoenergetic electrons are scattered from a low density of molecules and their final energy spectrum mimics the resonant energy structure of the scatterer. The correspondence of photoabsorption to the energy loss of scattered electrons relies on the incident beam being sufficiently energetic and the energy-loss spectrum being recorded at small scattering angles (Inokuti 1971). The lack of spectral resolution in this kind of experiment does not lead to an underestimate of unresolved features, in contrast to direct photoabsorption measurements, because of the linear relation between cross section and the signal from the analysed electrons. Electron-energy-loss cross sections can be recorded for energylosses spanning the entire photoabsorbing wavelength range and then absolutely calibrated according to the Thomas-ReicheKuhn sum rule (Backx et al. 1976) without detailed knowledge of the sample column density. These kinds of experiments typically yield an uncertainty of $30 \%$ or better but can not resolve the detailed wavelength structure of many molecules. They provide a useful comparison to benchmark the accuracy of higherspectral-resolution direct absorption cross sections.

The absorption of a photon with energy greater than the ionisation energy of a molecule can produce charged fragments. The resultant photoions and photoelectrons can be experimentally manipulated with electric fields and detected with high-efficiency, possibly simultaneously (e.g., Backx et al. 1976; Holland et al. 1993; Edvardsson et al. 1999). When all fragments are simultaneously detected, it is possible to spectroscopically examine the initial neutral species and produced ions. The simultaneous recording of photoion or photoelectron, and photoabsorption spectra provides a direct measurement of the fraction of excited molecules that decay via ionisation versus dissociation. Most molecules are ionised with near-100\% efficiency by photons more than about $2 \mathrm{eV}$ above their ionisation thresholds.

The branching to different dissociative-ionisation channels follows from the discrimination of photofragments with different charge-to-mass ratios. Less commonly, experimenters count neutral photofragments (e.g., Morley et al. 1992; Walter et al. 1993; Gao et al. 2013) or detect their fluorescence following dissociation into excited states (e.g., Lee 1984; Biehl et al. 1994). The emission of photoexcited molecules has is sometimes recorded (e.g., Jonas et al. 1990; Heays et al. 2014a) and provides further information on the decay branching of excited states.

\subsection{Theoretical cross sections}

\subsubsection{General considerations}

Quantum-chemical calculations of the excited electronic states of atoms and small molecules can be used to simulate photoabsorption, dissociation, and ionisation cross sections, and are particularly useful for species that are difficult to measure in the laboratory, such as radicals and ions (see Kirby \& van Dishoeck 1988; van Dishoeck 1988; van Dishoeck \& Visser 2015, for reviews).

For molecules, such calculations require knowledge of the ground state, one or more excited states, and the transition dipole moment connecting them. Ground and excited states are frequently summarised by potential energy curves describing the electrostatic interaction energy of the electrons as a function of the nuclear configuration. Some of these are plotted in Fig. 3 for the 1-dimensional case of a diatomic molecule, $\mathrm{O}_{2}$. These states are labelled by their symmetry and a numerical label increasing with excitation energy. For example, the $2^{1} \Sigma^{-}$state denotes the second state of ${ }^{1} \Sigma^{-}$symmetry. If this state has been observed experimentally, it often also has an alphabetic label, with the letters; A, B, C, ...; mostly increasing with excitation energy. For polyatomic molecules, the notation becomes $\tilde{A}, \tilde{B}, \tilde{C} \ldots$

The ground state potential energy of a stable molecule must form a well, leading to a quantised spectrum of bound states with increasing vibrational excitation. Electronically-excited states may be bound or repulsive, that is, possess no minimum energy (see Figs. 2 and 3). As discussed above, this distinction dramatically affects the structure of the resultant photoabsorption spectrum. The example in Fig. 3 reproduces the most important ultraviolet-excited states of $\mathrm{O}_{2}$ (Lewis et al. 1998, 2001) alongside its photoabsorption cross section. The strength of the 
cross section into each excited state depends on its specific transition moment with the ground state, and the size of the overlap of ground and excited vibrational wavefunctions. Within the Born-Oppenheimer approximation, this second factor requires a separate calculation considering the movement (vibration) of nuclei in a precomputed potential-energy environment.

The effects of nonzero molecular rotation are not usually explicitly included in ab initio cross section calculations, but can be simulated by assigning standard rotational-line strength factors (Larsson 1983) and assuming a population distribution of ground state rotational levels. These factors are not always accurate if centrifugal effects significantly alter the vibrational overlap of ground and excited states or the dissociation efficiency (e.g,. Lewis et al. 2005; Heays et al. 2011).

The spectral width of absorption features is characteristic of the lifetime of the excited state. The 135 to $180 \mathrm{~nm}$ absorption of $\mathrm{O}_{2}$ is rapidly followed by dissociative decay into $\mathrm{O}$ atoms, after less than $1 \mathrm{ps}$. The bound states at higher energy survive longer, but still dissociate because of a second-order interaction induced by the shown curve-crossing with the dissociative state (Lewis et al. 2002). States that take sufficiently long to dissociate, greater than typical Einstein $A$ coefficients of about $1 \mathrm{~ns}$, will have time to decay radiatively by spontaneous emission. Strong interactions lead to dissociation rates faster than $10^{12} \mathrm{~s}^{-1}$, implying a $100 \%$ dissociation efficiency. Detailed studies of the time evolution of nuclear motion may then provide an estimate of the dissociation branching ratio of photoexcited states (e.g., van Dishoeck et al. 1984; Kroes et al. 1997; Lewis et al. 2002; Heays et al. 2011).

The intrinsic linewidths of absorption features are given by the inverse of the sum of the predissociation and spontaneous decay rates, $1 /\left(k^{\text {pre }}+A\right)$. A predissociation rate $k^{\text {pre }}$ as large as $10^{11} \mathrm{~s}^{-1}$ corresponds to a linewidth of $5 \times 10^{-4} \mathrm{~nm} \mathrm{FWHM}$ (full-width half-maximum) at a wavelength of $100 \mathrm{~nm}$. In velocity units, this amounts to $1 \mathrm{~km} \mathrm{~s}^{-1}$, which is comparable or less than the typical turbulent Doppler broadening of an interstellar clouds. Intrinsic widths seen in experimental data can vary greatly, from Doppler-broadening dominated (e.g., $\mathrm{N}_{2}$ ) to greater than $1 \mathrm{~nm}$ (e.g., $\mathrm{NH}_{3}$ and $\mathrm{C}_{2} \mathrm{H}_{2}$ ), obscuring all rotational structure when strongly predissociated. Such accurate knowledge of absorption line profiles is however only needed (i) to determine overlap with specific lines that dominate the radiation field in some astrophysical environments such as Lyman- $\alpha$; (ii) to compute optical depth and self-shielding capacity.

For polyatomic molecules, the calculation of multidimensional excited-state potential-energy surfaces including all degrees of freedom, and subsequent nuclear dynamics on those surfaces, becomes computationally prohibitive. Moreover, such detail is often not needed to compute accurate photodestruction rates since the necessary absorption strengths are largely determined by one or a few excited states and, for cold molecules, the relevant nuclear motion only probes a small region of coordinate space around the ground state equilibrium geometry. Therefore, a simpler alternative is to only compute vertical excitation energies and transition dipole moments defined at the equilibrium geometry, and assume a dissociation probability for the excited state. This reduces the photoabsorption cross section of an entire electronic transition to a single wavelength, whereas the real cross section may be very broad. This approximation is quite sufficient for the case of photodissociation in a continuum-like radiation field, for example, the ISRF.

Our database includes vertical-excitation cross sections computed for a number of molecules and summarised in van Dishoeck (1988), van Dishoeck et al. (2006) and van Hemert \& van Dishoeck (2008), based on our work and that of other groups (e.g., Kirby \& van Dishoeck 1988; Roueff et al. 2014). These results are based on high-level configuration interaction calculations (see van Dishoeck \& Visser 2015, for a top level overview of such calculations). In the latest calculations by van Hemert \& van Dishoeck (2008), up to 9 electronic states per symmetry are considered, including diffuse (Rydberg) states. For the lower-energy states, comparisons with independent calculations and experiments indicate that the deduced excitation energies are accurate to better than $0.3 \mathrm{eV}$ and that oscillator strengths connecting them to the ground state agree within $30 \%$ or better. For the higher states, typically the 5th root and higher per symmetry, the accuracy decreases because many states and orbitals can mix. Such calculations still provide a good indication of the location of those states and their combined strengths, typically within a factor of 2 .

Only states above the ground-state dissociation limit and below the ionisation potential of the molecule need be taken into account for photodissociation calculations. The dissociation efficiency, $\eta_{\mathrm{d}}$, of all calculated excited states in this range and presented here is assumed to be unity, that is, they are purely repulsive and dissociate directly, or have resonant levels and decay by predissociation (exceptions are $\mathrm{H}_{2}, \mathrm{CO}$ and $\mathrm{N}_{2}$ for which level-specific probabilities are available). For larger molecules (i.e., three or more atoms) dissociation rates assuming unity efficiency should be regarded as upper limits, given that internal conversion to a lower (dissociative) electronic state is usually much more rapid than radiative decay, because of their high density of states (e.g., Leger et al. 1989; Jochims et al. 1994). Above the ionisation potential, all absorption is assumed to lead to photoionisation (dissociative or not). Also, only states lying below the Lyman limit of $13.6 \mathrm{eV}$ are included.

Even after computing a full potential-energy surface the wavelengths and absorption oscillator strengths of known bound vibrational levels, their predissociation lifetimes and widths may still be unknown. Additionally, the real photoabsorption cross section into a bound vibrational level may involve multiple rotational transitions, effectively increasing the width of its photoabsorption envelope. We assumed a Gaussian profile to encompass these phenomena for theoretical predissociated levels used in our cross section database, and uniformly assumed a width of $1 \mathrm{~nm}$ FWHM, where our following calculation of interstellar photodissociation rates is not sensitive to the precise value of this width.

The accuracy of cross sections derived from ab initio calculations can be remarkably high, within $20 \%$ or better, for diatomic molecules (e.g., OH van Dishoeck \& Dalgarno 1983; van Dishoeck \& Dalgarno 1984a) and sometimes for polyatomic cases (e.g., $\mathrm{H}_{2} \mathrm{O}$ in Sect. 4.3.33). The wavelengths of absorption lines exciting predissociated bound levels may be significantly in error where non-Born-Oppenheimer interactions shift energy levels and redistribute oscillator strengths between excited states (e.g., van Dishoeck et al. 1984). However, inaccuracies introduced by these effects are much reduced in the calculation of interstellar photodissociation rates that average over many states (e.g., $\mathrm{C}_{3} \mathrm{H}$ in Sects. 4.3.25 and 4.3.26). The largest uncertainty in ab initio photodissociation cross sections then arises, in most cases, from inaccurately-calculated or neglected states lying close to the ionisation threshold, which are numerous and difficult to calculate or measure.

Empirical corrections can resolve some of the uncertainty of theoretical cross sections, either by shifting absorption features to their experimentally known wavelengths, or adjusting the underlying excited state potential-energy surfaces to 
produced cross sections in better agreement with experiment (e.g., Heays et al. 2014a). For a few molecules in our database, we added a guessed wavelength and integrated cross section to approximate the influence of neglected high-lying states, with an associated order-of-magnitude uncertainty (in general, these additions contribute a small amount to the overall cross section and its uncertainty). For reference, inclusion of a hypothetical state at $9 \mathrm{eV}$ with an oscillator strength of 0.1 would increase the ISRF photodissociation rates by $3.5 \times 10^{-10} \mathrm{~s}^{-1}$. In general, no corrections were made for possibly-neglected states above the ionisation limit and below $13.6 \mathrm{eV}$. This is because the lowest Rydberg members are generally computed explicitly, and the oscillator strengths of higher Rydberg states converging to the ionisation threshold decrease roughly as $1 / n^{3}$ ( $n$ is the principal quantum number) and do not contribute much.

For all theoretical cross sections in our database, a minimum photodissociation cross section of $5 \times 10^{-20} \mathrm{~cm}^{2}$ was assumed between the dissociation threshold and Lyman-limit at $91.2 \mathrm{~nm}$. This weak continuum negligibly increases the integrated cross section but ensures a low but nonzero cross section overlaps the strong emission lines present in some interstellar radiation fields.

\subsubsection{Atomic photoionisation}

Atomic photoionisation cross sections have long been an object of theoretical study due to their influence on the interpretation of spectroscopic observations of astrophysical plasmas in ionised interstellar gas as found around stars, active galactic nuclei, and elsewhere (Seaton 1951; Osterbrock 1979; Ferland 2003; Tielens 2013). We used theoretical cross sections here for the photoionisation of some neutral atoms. These are generally the result of $R$-matrix calculations (Seaton 1985; Mendoza 1996; McLaughlin 2001; Zatsarinny \& Bartschat 2013), and produce continuum cross sections that are generally accurate to within $20 \%$. The specification of resonant structure evident in most atomic cross sections presents more difficulty for this method, although the uncertainties are diminished for photoionisation rates calculated following integration over many resonances.

\subsection{Cross section databases}

There are various public databases of photoabsorption, dissociation, and ionisation cross sections, and some data from these were incorporated into our assessment of molecular and atomic cross sections. A comprehensive set of laboratory photoabsorption cross sections and a smaller amount of data concerning photofragment branching ratios is contained in the MPI Mainz UV/VIS Spectral Atlas ${ }^{4}$. Earlier compilations are given by Calvert \& Pitts (1966), Okabe (1978), Lee (1984), Gallagher et al. (1988), Ashfold et al. (2006). The TOPbase database of photoionisation cross sections (Mendoza 1996) includes $R$-matrix calculations for many atoms, including their highly-charged states. A collation of molecular and atomic cross sections from multiple sources is contained in the PHIDRATES database $^{6}$ (Huebner et al. 1992; Huebner \& Mukherjee 2015) as well as calculations of their photodissociation and photoionisation rates in the ISRF and solar radiation fields. Our compilation differs somewhat from Huebner \& Mukherjee (2015) for molecules in common, due to different choices of cross section data and a larger focus on highly excited electronic states in our

\footnotetext{
satellite.mpic.de/spectral_atlas

cdsweb.u-strasbg.fr/topbase/topbase.html

6 phidrates.space.swri.edu
}

work that are more important for the ISRF than for the solar radiation field.

More specialised databases containing cross sections of astrochemical interest are the MOLAT and SESAM databases of vacuum-ultraviolet (VUV) spectroscopy ${ }^{7}$, including $\mathrm{CO}, \mathrm{H}_{2}$, and $\mathrm{N}_{2}$; the Harvard CfA VUV database ${ }^{8}$ including primary data on many small molecules including wavelengths as short as $80 \mathrm{~nm}$; and the UGA Opacity Project database ${ }^{9}$. The VAMDC virtual portal ${ }^{10}$ integrates some of these data.

\section{Compiled cross sections}

In this section the cross sections of atoms and molecules in our database are presented. All cross sections are plotted in Figs. 4 to 14 and have some summarised properties listed in Table 1. Complete descriptions of the source material for most cross section are given in Sects. 4.3.1 to 4.3.72. For some species, we did not exhaustively reappraise the literature and instead give a reference to its cross section in Table 1 . There are dissociation and ionisation thresholds listed in Table 1 for all species where these are relevant. In some cases the listed molecular dissociation limits correspond to greater photon energies (shorter wavelengths) than the dissociation energies of their ground electronic states, due to the lack of accessible excited states for photoabsorption at these energies.

\subsection{Cross section uncertainties}

We assigned uncertainties to each overall molecular and atomic cross section according to estimates within their source material, where available, or based on the general accuracy of the various experimental and theoretical methods used, as discussed in Sects. 3.2 and 3.3.

We limited our estimated uncertainties to four broad categories for simplicity and in view of the ubiquity of large uncertainties in many other key parameters in astrophysical modelling. These categories are:
$\mathrm{A}^{+}$: accurate to within $20 \%$;
A: accurate to within $30 \%$;
B: accurate within a factor of 2 ;
C: accurate within a factor of 10 .

For the purposes of programs requiring uncertainties in terms of log-normal standard deviations, our rating system corresponds approximately to $2 \sigma$ uncertainties.

For some molecules, the compilation of data sources into a single best estimated cross section introduces clear wavelengthdependence into the cross section uncertainty, which we weighted according to the wavelength dependence of the ISRF to give the estimates in Table 1 . Then, a greater uncertainty at the shortest wavelengths will not contribute as much to our uncertainty estimate as near the long-wavelength threshold.

The uncertainty of photodestruction rates calculated according to Eq. (1) will potentially differ for non-ISRF radiation fields. This is most significant for the case of a Lyman- $\alpha$ dominated radiation field, where the cross section uncertainty at $121.6 \mathrm{~nm}$ is most important. For molecules with weak and uncertain continua

\footnotetext{
7 molat.obspm.fr and sesam.obspm.fr

8 wWW.cfa.harvard.edu/amp/ampdata/cfamols.html

9 www.physast.uga.edu/ugamop/index.html

10 portal. vamdc.org
} 
A. N. Heays et al.: Photodissociation and photoionisation of atoms and molecules of astrophysical interest

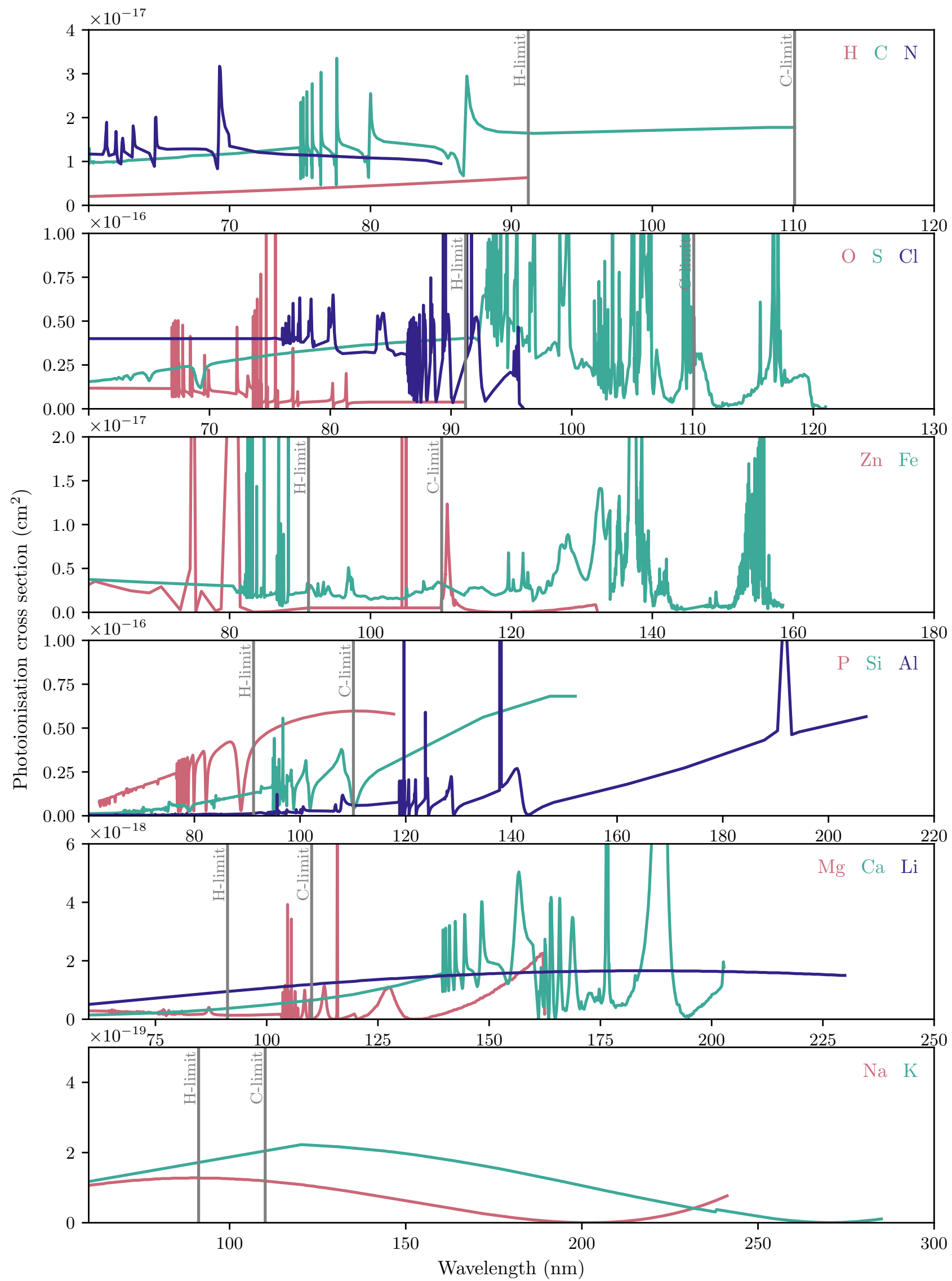

Fig. 4. Compiled atomic photoionisation cross sections. The ionisation thresholds of $\mathrm{H}$ and $\mathrm{C}$ are indicated by vertical lines. 

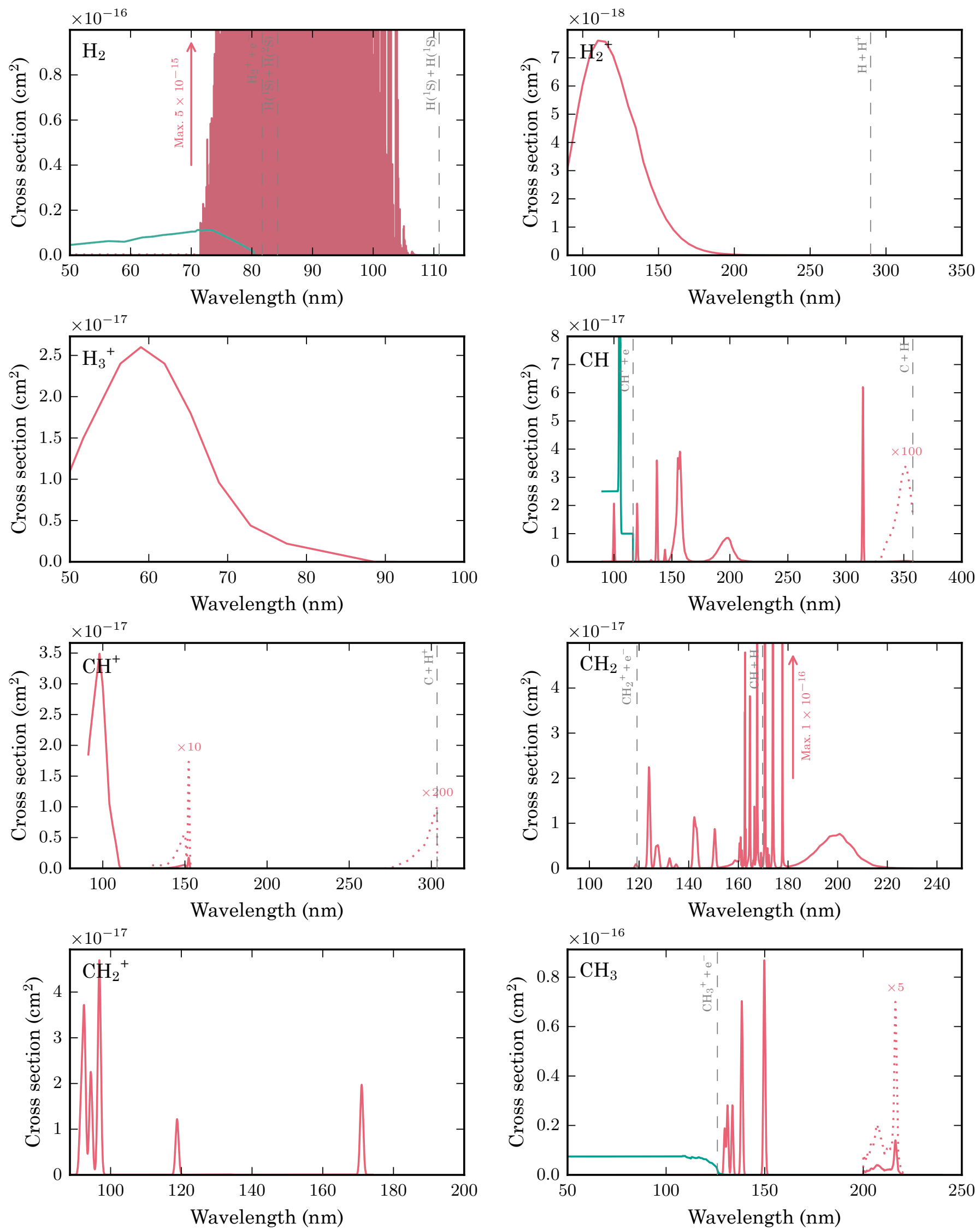

Fig. 5. Cross sections of molecules. Red: photodissociation. Blue: photoionisation. Some photofragmentation thresholds are also labelled. 
A. N. Heays et al.: Photodissociation and photoionisation of atoms and molecules of astrophysical interest
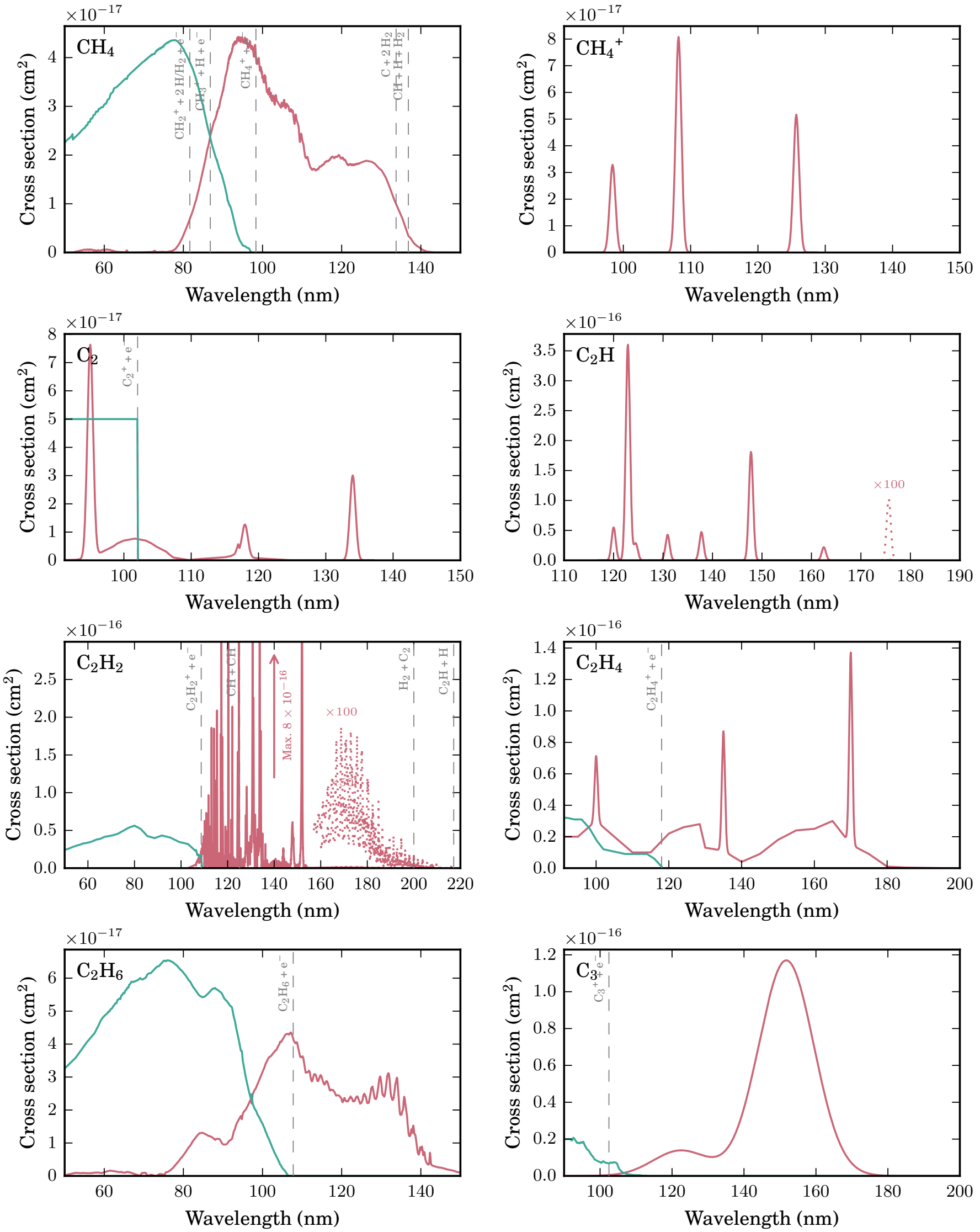

Fig. 6. Cross sections of molecules. Red: photodissociation. Blue: photoionisation. Some photofragmentation thresholds are also labelled. 

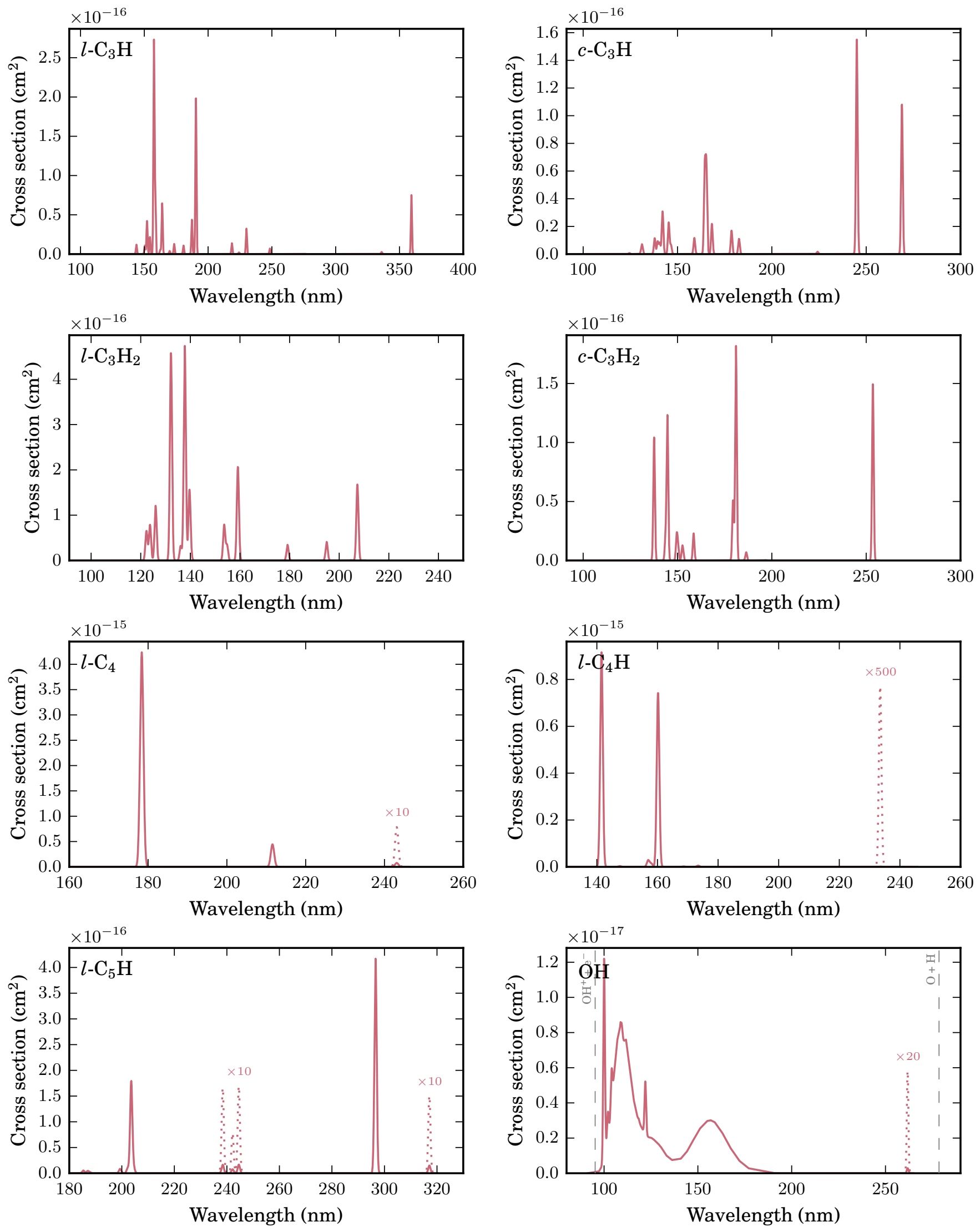

Fig. 7. Cross sections of molecules. Red: photodissociation. Blue: photoionisation. Some photofragmentation thresholds are also labelled. 
A. N. Heays et al.: Photodissociation and photoionisation of atoms and molecules of astrophysical interest
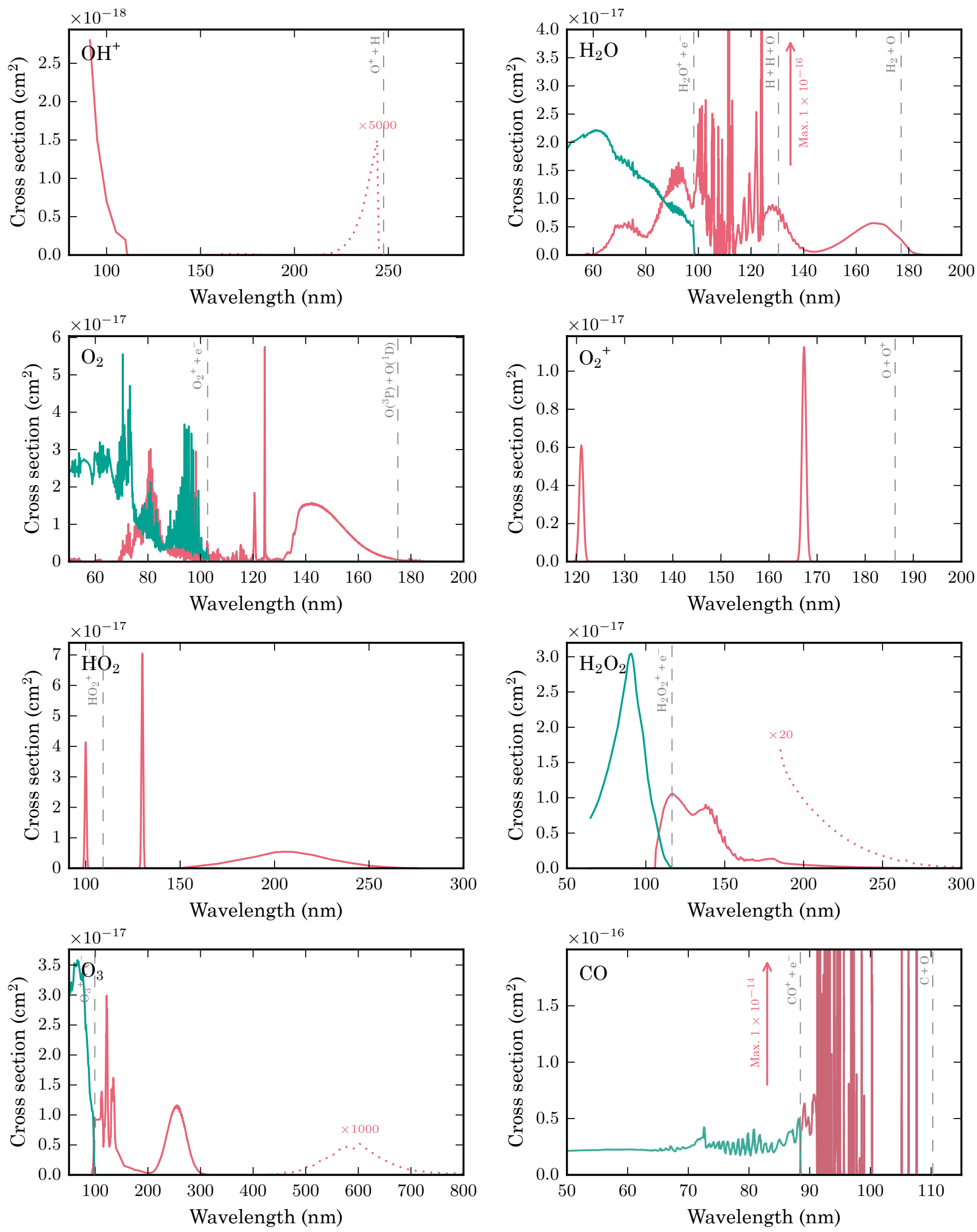

Fig. 8. Cross sections of molecules. Red: photodissociation. Blue: photoionisation. Some photofragmentation thresholds are also labelled. 

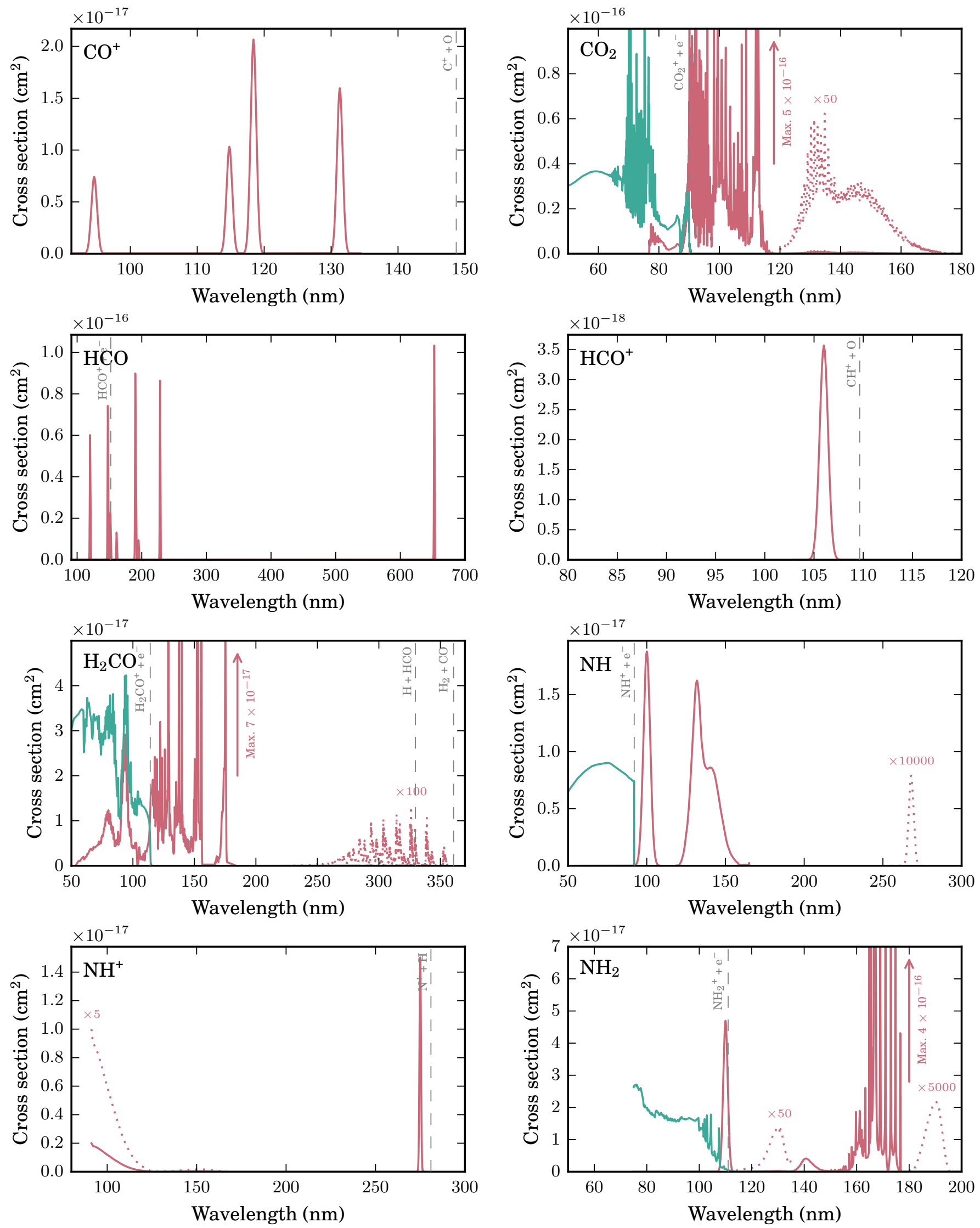

Fig. 9. Cross sections of molecules. Red: photodissociation. Blue: photoionisation. Some photofragmentation thresholds are also labelled. 
A. N. Heays et al.: Photodissociation and photoionisation of atoms and molecules of astrophysical interest
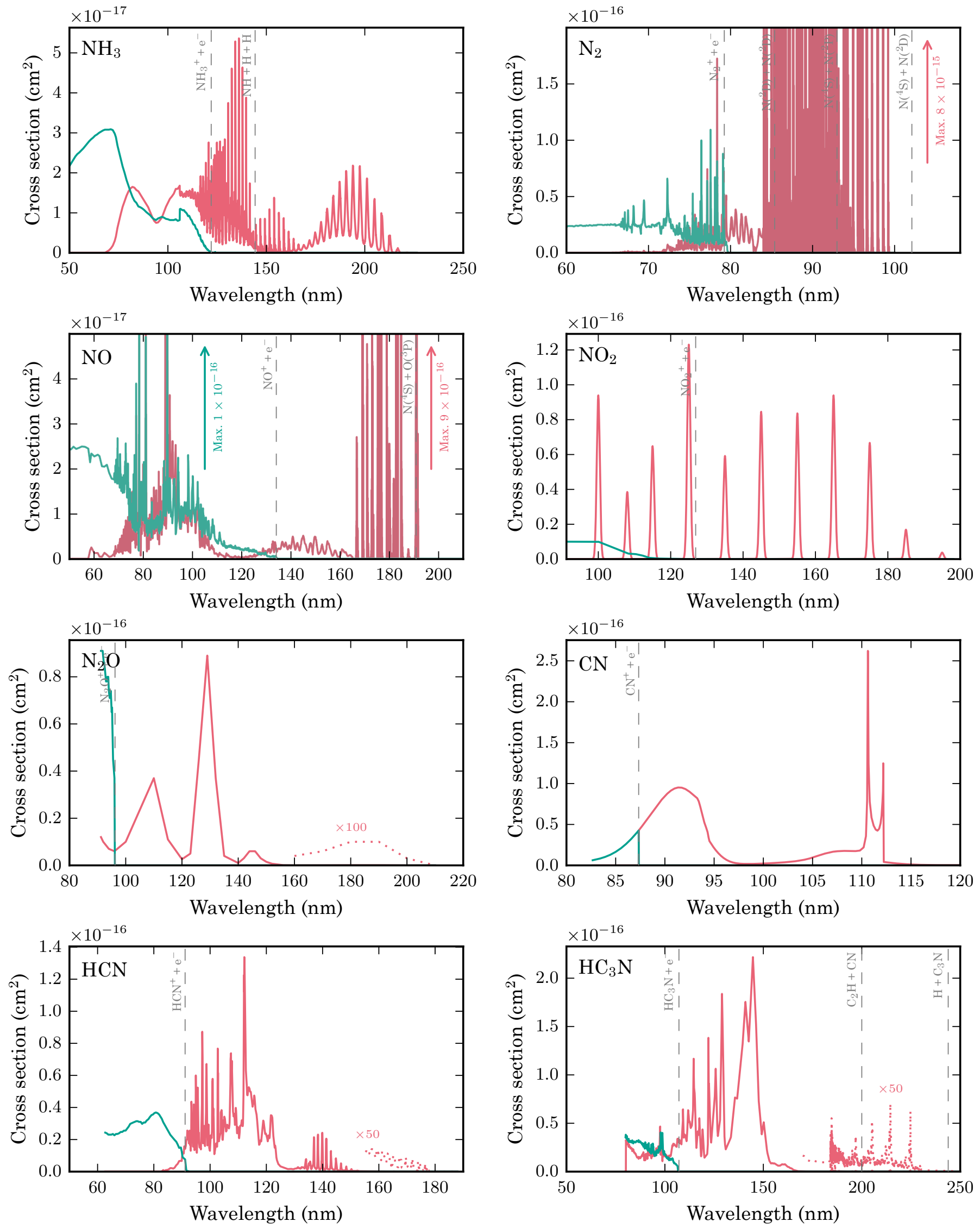

Fig. 10. Cross sections of molecules. Red: photodissociation. Blue: photoionisation. Some photofragmentation thresholds are also labelled. 

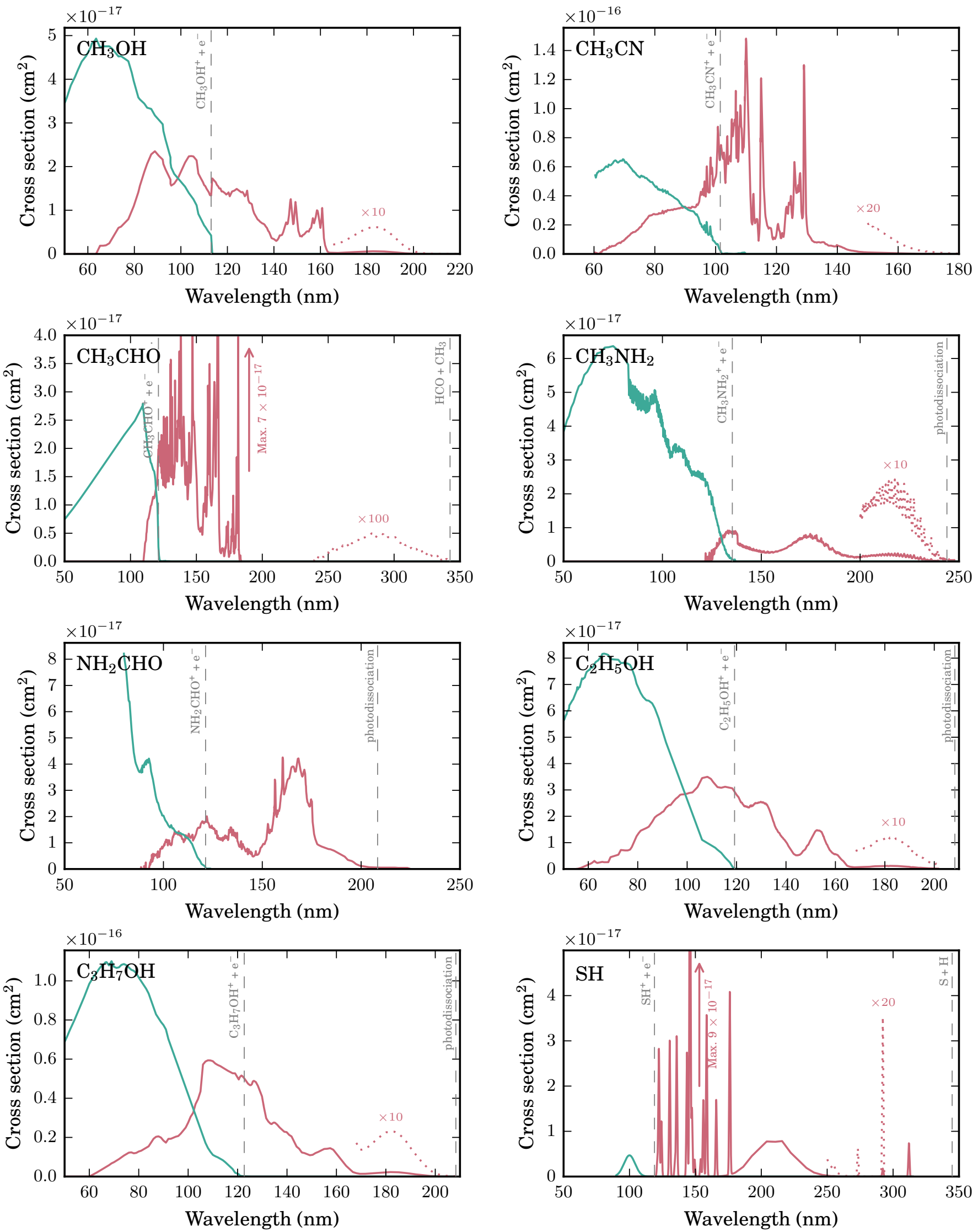

Fig. 11. Cross sections of molecules. Red: photodissociation. Blue: photoionisation. Some photofragmentation thresholds are also labelled. 
A. N. Heays et al.: Photodissociation and photoionisation of atoms and molecules of astrophysical interest
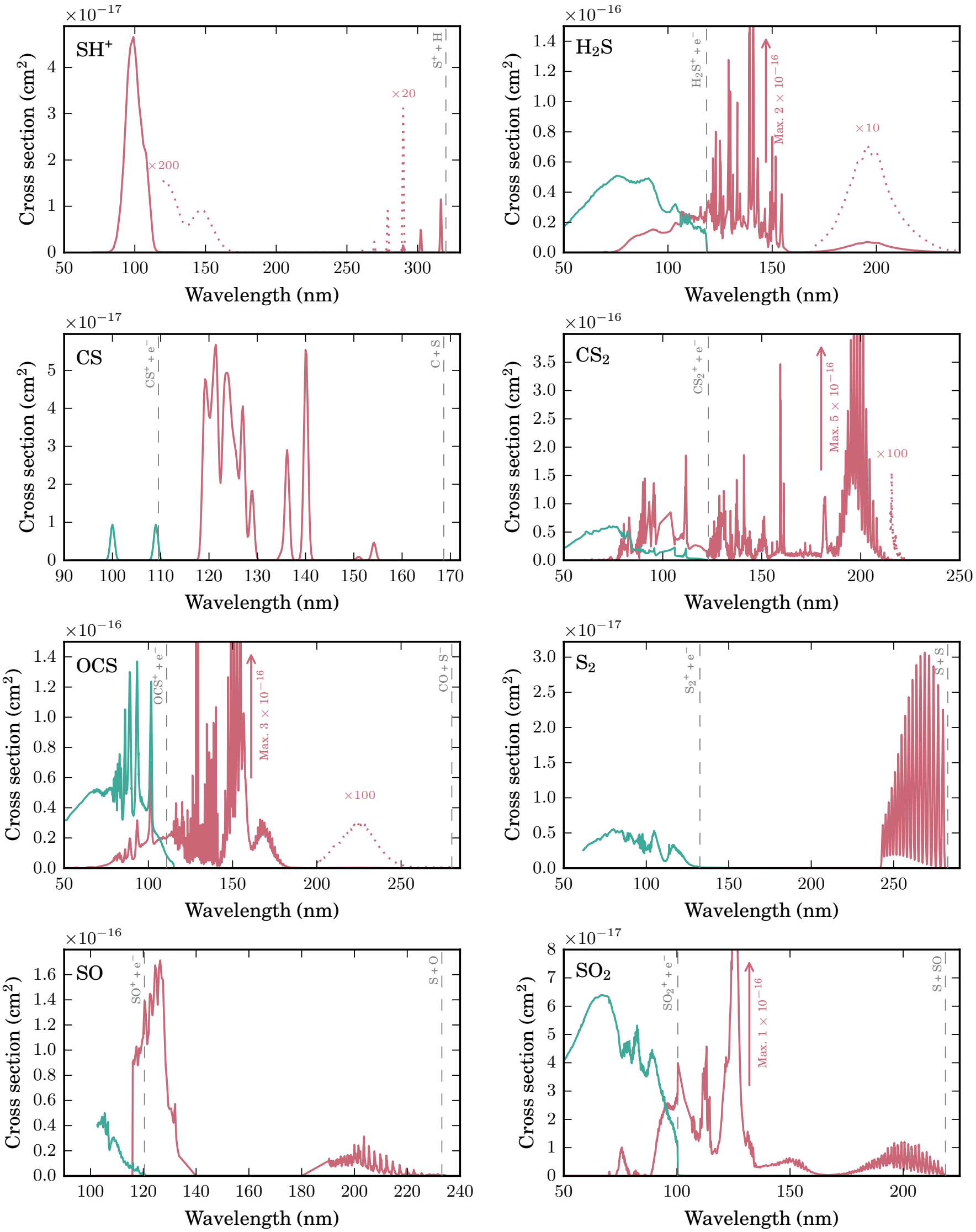

Fig. 12. Cross sections of molecules. Red: photodissociation. Blue: photoionisation. Some photofragmentation thresholds are also labelled. 

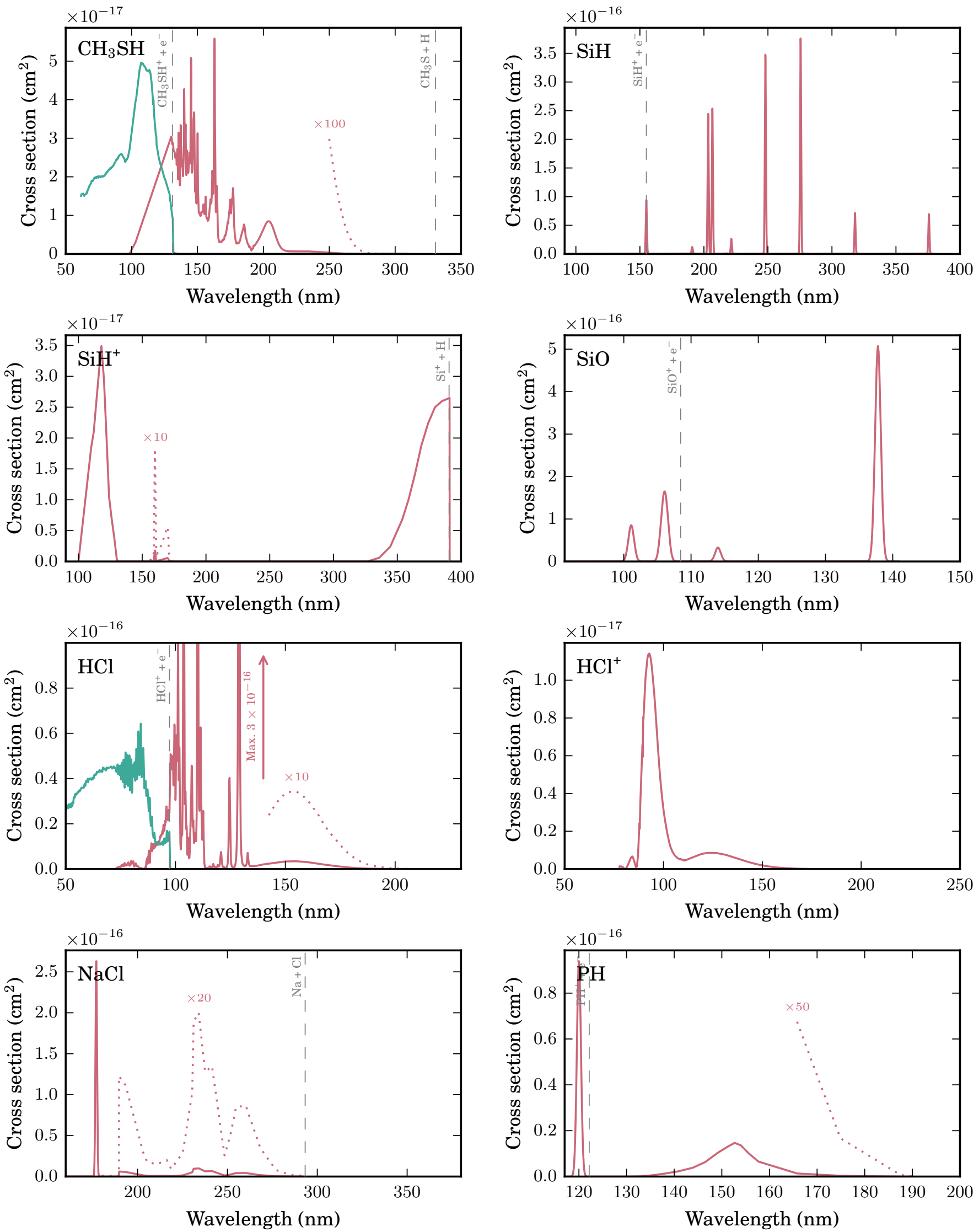

Fig. 13. Cross sections of molecules. Red: photodissociation. Blue: photoionisation. Some photofragmentation thresholds are also labelled. 
A. N. Heays et al.: Photodissociation and photoionisation of atoms and molecules of astrophysical interest

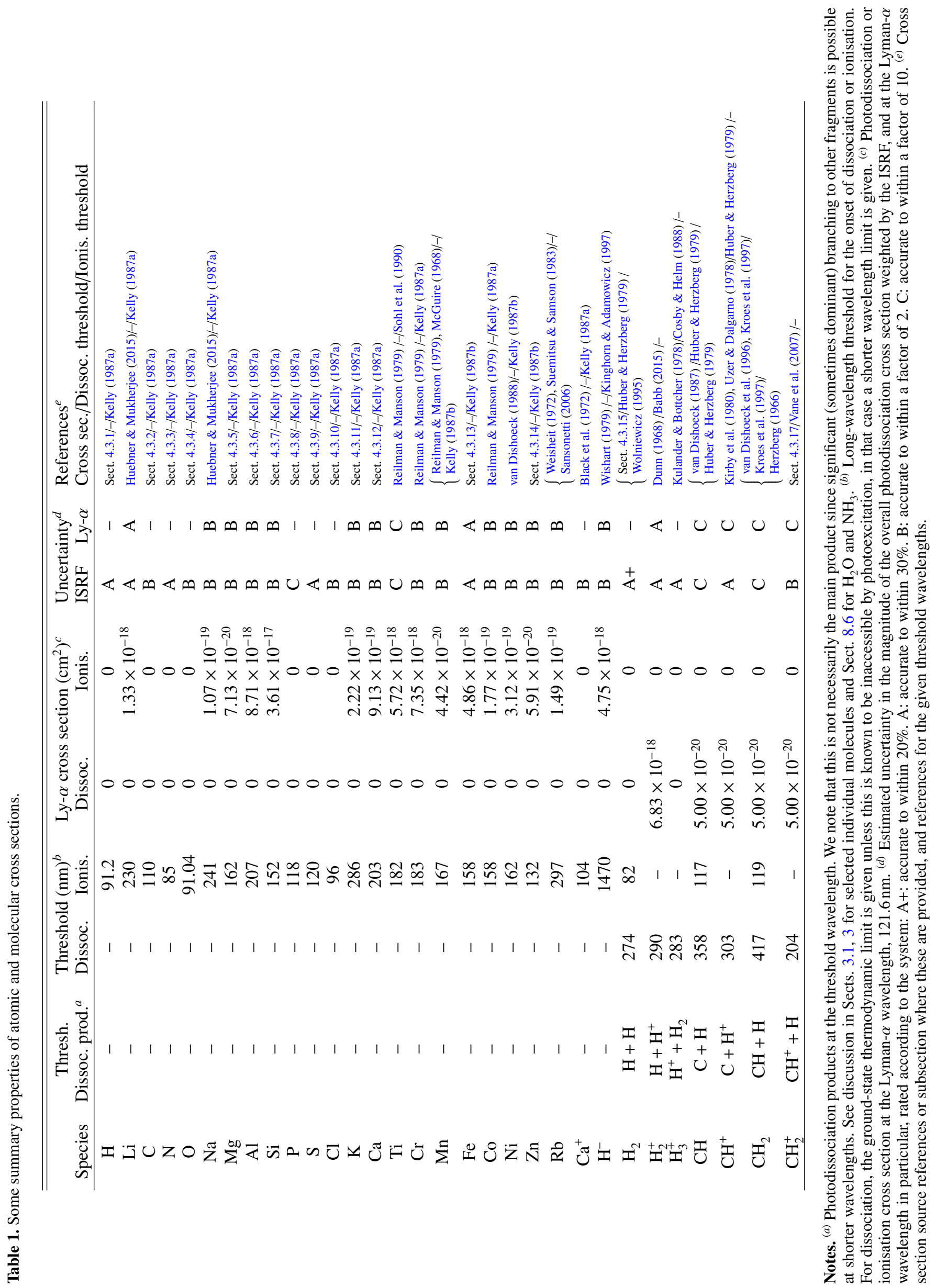




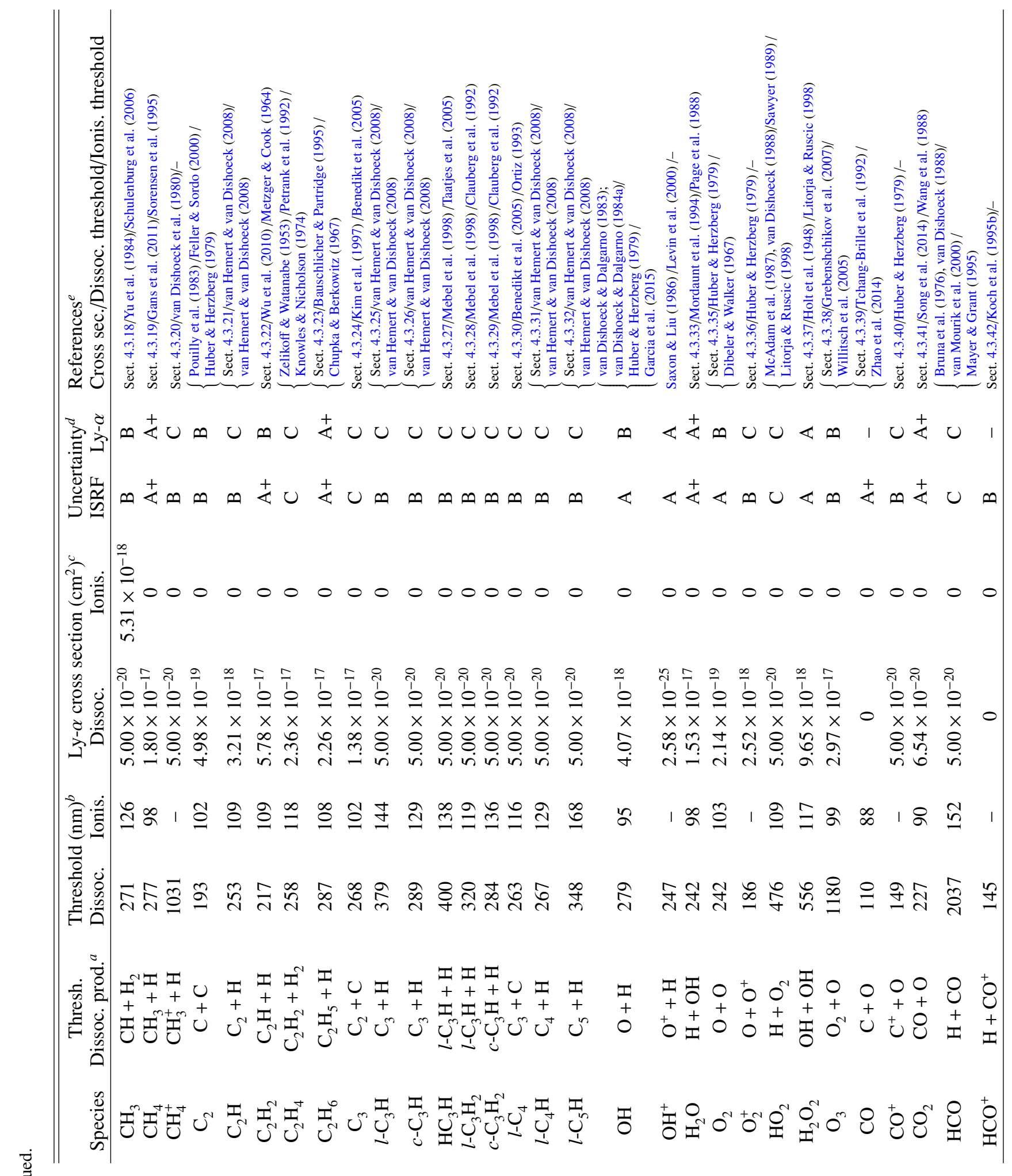




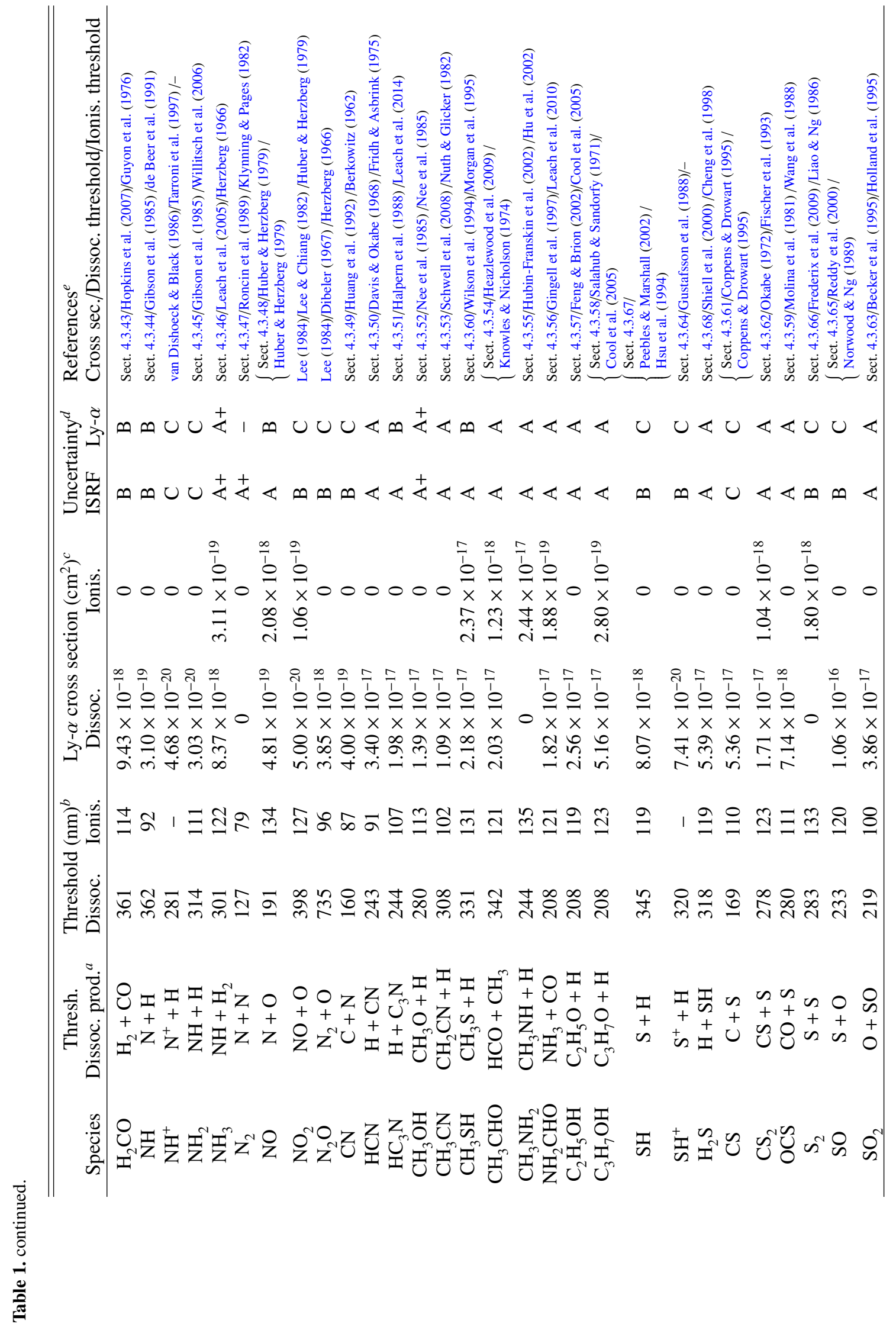




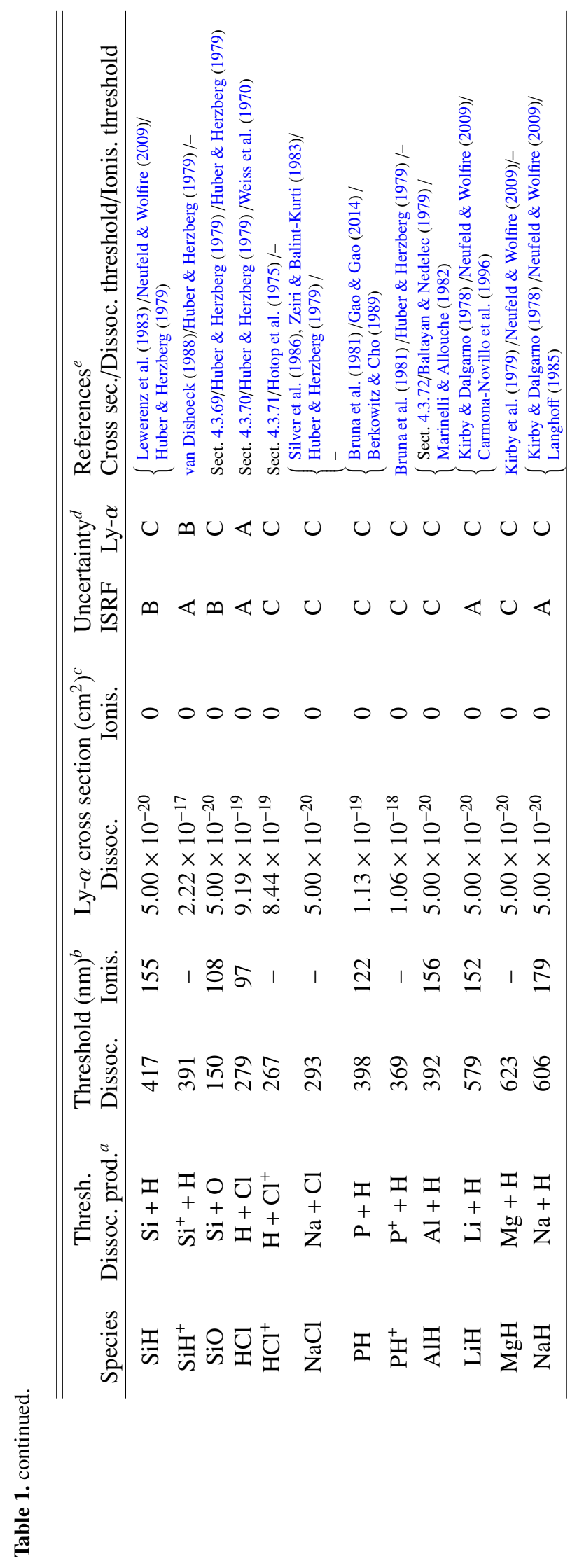


A. N. Heays et al.: Photodissociation and photoionisation of atoms and molecules of astrophysical interest
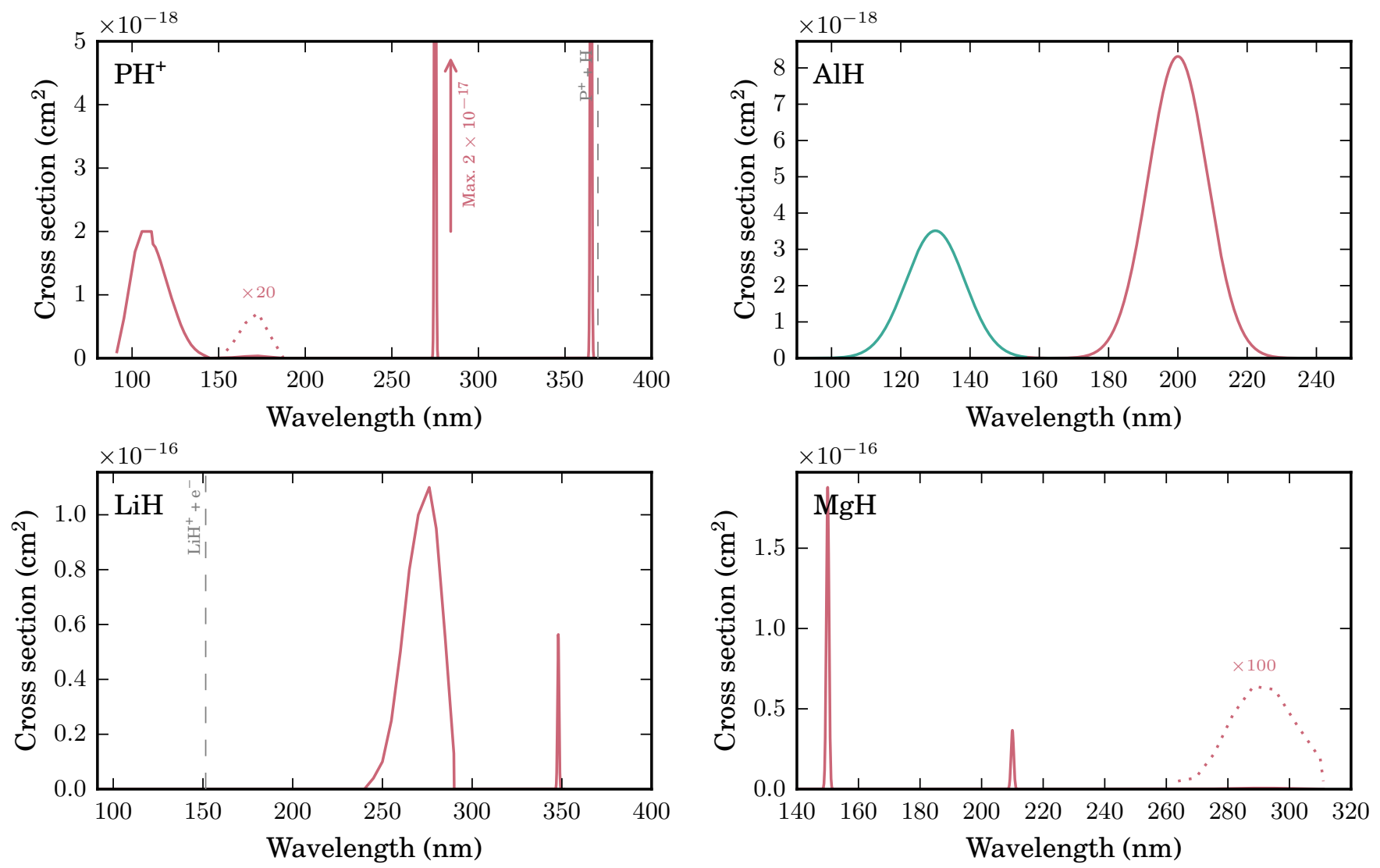

Fig. 14. Cross sections of molecules. Red: photodissociation. Blue: photoionisation. Some photofragmentation thresholds are also labelled.

at this wavelength, or sharply resonant structure that may be experimentally under-resolved, recorded at an inappropriate temperature, or calculated with a line position error, the uncertainty may be significantly larger than for the overall cross section. For this reason we provide a separate uncertainty rating applicable to the Lyman- $\alpha$ wavelength.

The comparison of independent cross section measurements and calculations allows for testing their claimed uncertainties. An example is $\mathrm{H}_{2} \mathrm{O}$ in Sect. 8.4, where we find that the ISRF photodissociation rate obtained with cross section data from four independent experiments with quite different methodologies agrees within $10 \%$.

\subsection{Cross section database format}

The collected cross sections, with references, are available for download from the Leiden Observatory database ${ }^{11}$. The data files are given in two alternative formats for convenient utilisation, in text files listing the continuum and line absorption of each cross section separately, and in binary format encoding the full wavelength dependence of absorption lines.

The first case provides for a concise data format and rapid calculation of the photodissociation rates for line absorption in a continuum field. This is at the cost of information regarding their shapes and its overlap with a structured radiation field. The strengths of discrete absorption lines are represented by their integrated cross section; related to the well-known (dimensionless) oscillator strength, $f_{u \ell}$ for a transition between upper state $u$ and lower state $\ell$; and defined as follows,

$$
\begin{aligned}
\sigma^{\mathrm{int}} & =\int \sigma(\lambda) \mathrm{d} \lambda \\
& =\frac{e^{2}}{4 \epsilon_{0} m_{\mathrm{e}} c^{2}} \lambda_{0}^{2} x_{\ell} f_{u \ell} \eta^{d}, \\
& =8.85 \times 10^{-20} \lambda_{0}^{2} x_{\ell} f_{u \ell} \eta^{d} .
\end{aligned}
$$

Here, the final form is appropriate for case of $\sigma$ and $\lambda$ in units of $\mathrm{cm}^{2}$ and $\mathrm{nm}$, respectively. The integration domain for the cross sections is the wavelength range of an absorption feature, whether it is an individual ro-vibrational transition, or an entire electronic-state transition containing many rotational-vibrational levels. Here, $e, m_{\mathrm{e}}, c$, and $\epsilon_{0}$ are the electron charge and mass, the speed of light, and permittivity of free space, respectively, and $\lambda_{0}$ is the central wavelength of the transition. Finally, $\eta^{\mathrm{d}}$ is the dissociation probability of the upper level and $x_{\ell}$ is a ground-state fractional population. The latter is 1 for oscillator strengths describing transitions between entire electronic states but will depend on the ground state excitation temperature when individual rotational or vibrational transitions are considered.

Then, the photodissociation or ionisation rate defined in Eq. (1) is substituted by the equation,

$k=\int \sigma^{\text {cont }}(\lambda) I(\lambda) \mathrm{d} \lambda+\sum_{i=1}^{\text {all lines }} I\left(\lambda_{0}\right) \sigma^{\mathrm{int}}$.

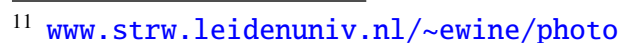


As an example the photodissociation cross section of the $\mathrm{CH}_{2}$ radical is available as the data file ch2 .pd with contents:

\begin{tabular}{rcc} 
CH2 P.D. Cf. van Dishoec \\
17 \\
1 & 1187.6 & $0.100 \mathrm{E}-16$ \\
2 & 1227.6 & $0.800 \mathrm{E}-19$ \\
3 & 1240.0 & $0.240 \mathrm{E}-15$ \\
4 & 1267.7 & $0.460 \mathrm{E}-16$ \\
5 & 1276.9 & $0.490 \mathrm{E}-16$ \\
\multicolumn{4}{c}{ (more } & data) \\
13 & 1433.3 & $0.540 \mathrm{E}-16$ \\
14 & 1504.7 & $0.920 \mathrm{E}-16$ \\
15 & 1585.5 & $0.620 \mathrm{E}-17$ \\
16 & 1595.7 & $0.450 \mathrm{E}-18$ \\
17 & 1680.0 & $0.200 \mathrm{E}-16$ \\
306 & & \\
-2750. & & \\
1 & 1427.6 & $0.100 \mathrm{E}-20$ \\
2 & 1516.3 & $0.100 \mathrm{E}-18$ \\
3 & 1555.7 & $0.499 \mathrm{E}-18$ \\
4 & 1564.1 & $0.684 \mathrm{E}-18$ \\
5 & 1572.6 & $0.870 \mathrm{E}-18$ \\
& $(m o r e$ & data) \\
302 & 2388.4 & $0.733 \mathrm{E}-21$ \\
303 & 2408.2 & $0.707 \mathrm{E}-21$ \\
304 & 2428.4 & $0.173 \mathrm{E}-21$ \\
305 & 2448.9 & $0.361 \mathrm{E}-21$ \\
306 & 2750.0 & $0.000 \mathrm{E}+00$
\end{tabular}

This file can be serially decoded as follows:

Line 1: describes the contents of the data file, provides the main literature reference.

Line 2: number of discrete lines through which photodissociation occurs for this molecule, $n_{l}$.

Line 3 to $3+n_{l}$ :

Field 1: index of a line;

Field 2: wavelength of the line centre $(\AA)$;

Field 3: integrated cross section of this line $\left(\mathrm{cm}^{2} \AA\right)$.

Line $3+n_{l}+1$ : number of continuum points defined, $n_{c}$.

Line $3+n_{l}+2$ : long wavelength threshold for the continuum data, if set to -1 or blank the last explicitly listed wavelength is used.

Line $3+n_{l}+3$ to $3+n_{l}+3+n_{c}$ :

Field 1: index of this point;

Field 2: wavelength $(\AA)$;

Field 3: cross section $\left(\mathrm{cm}^{2}\right)$.

All cross sections are also provided as data files in an alternative format including a full specification of their wavelength dependence. This may require tens or hundreds of thousands of wavelength points to capture the cross section of highlystructured molecules. To facilitate the handling of such large datasets we provide them in the hdf5 binary format ${ }^{12}$. For the case of $\mathrm{CH}_{2}$ with file name $\mathrm{CH} 2$. hdf5, this contains a dataset labelled README, providing a description of the file contents, literature references, and uncertainty estimate; a binary array labelled wavelength in units of $\mathrm{nm}$; and multiple molecular cross sections in units of $\mathrm{cm}^{2}$, labelled photoabsorption, photodissociation, and photoionisation. The discrete lines listed with integrated cross sections in the text-formatted files are included in the continuum-only binary files with Gaussian profiles of $1 \mathrm{~nm}$ FWHM. Astrophysically-important rates calculated in the following are not sensitive to the precise value of this assumed width.

12 wWw.hdfgroup.org

\subsection{Photodissociation and photoionisation of atoms and molecules}

In the following, the cross sections of atoms and molecules are discussed and summarised. These subsections (and various tables in the paper) are ordered by chemical type, for example, atoms, hydro-carbon species, and oxygen-, nitrogen-, sulphurand metal-containing molecules. A summary of dissociation and ionisation thresholds, cross sections at the Lyman- $\alpha$ wavelength, and the estimated uncertainty of these cross sections is provided in Table 1. The ordering of the following subsections follows the row ordering of Table 1 .

\subsection{1. $\mathrm{H}$ - hydrogen}

The hydrogen photoionisation continuum, $91.2 \mathrm{~nm}$ and shorter, is calculated for the TOPbase database (Mendoza 1996) and agrees very well with an experimental measurement (Palenius et al. 1976) where they overlap. We simulate the longer-wavelength Lyman-series line absorption from a list of transition wavelengths and oscillator strengths from the NIST atomic database ${ }^{13}$ (Kramida 2010). We adopted a Gaussianshaped Doppler broadening of $1 \mathrm{~km} \mathrm{~s}^{-1}$ to accompany the Lorentzian natural linewidths of the $\mathrm{H}$ lines in the simulated photoabsorption cross section.

\subsubsection{C - carbon}

The calculated cross section for $\mathrm{C}$ was taken from the TOPbase database (Mendoza 1996; Nahar \& Pradhan 1997).

\subsection{3. $\mathrm{N}$ - nitrogen}

The long-wavelength photoionisation threshold of atomic nitrogen, $85 \mathrm{~nm}$, is shorter than the Lyman-limit, $91.2 \mathrm{~nm}$, and its photoionisation in the interstellar medium is then suppressed. The cross section adopted here is taken from the absolute experimental data of Samson \& Angel (1990), apart from the resonant region between 61.8 and $71.5 \mathrm{~nm}$ where the relative-magnitude high-resolution measurement of Dehmer et al. (1974) was used, after scaling this to match the Samson \& Angel value at $67 \mathrm{~nm}$, in a continuum region.

\subsubsection{O - oxygen}

A theoretical calculation of the oxygen photoionisation cross section (Cantu \& Parkinson 1988; Huebner \& Mukherjee 2015) was used and is in good agreement with an absolute photoionisation yield (Angel \& Samson 1988) that lacks the spectral resolution to reveal the majority of calculated photoionisation resonances.

\subsection{5. $\mathrm{Mg}$ - magnesium}

The experimental photoabsorption cross section of Yih et al. (1998) was adopted for the photoionisation cross section of $\mathrm{Mg}$ for wavelengths between 120 and $163 \mathrm{~nm}$. At shorter wavelengths the calculated TOPbase cross section (Mendoza 1996) was used after rescaling by a factor of 1.14 to match the integrated value of Yih et al. (1998) where they overlap.

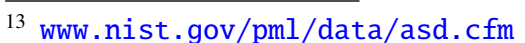




\subsubsection{Al-aluminium}

The calculated cross section for Al was taken from the TOPbase database (Mendoza 1996) and is in reasonable agreement with an absolutely-calibrated experimental measurement (Kohl \& Parkinson 1973).

\subsubsection{Si - silicon}

There are multiple R-matrix calculations of Si photoionisation (Mendoza \& Zeippen 1988; Nahar \& Pradhan 1993) that are broadly in agreement. Here, we adopt a cross section from the TOPbase database (Mendoza 1996; Huebner \& Mukherjee 2015).

\subsection{8. $\mathrm{P}$ - phosphorus}

The photoionisation cross section determined by Tayal (2004) from R-matrix calculations is adopted between 62 and $118 \mathrm{~nm}$.

\subsection{9. $\mathrm{S}-$ sulphur}

The recent R-matrix calculation of Barthel et al. (2015) is adopted for the atomic-S photoionisation cross section for wavelengths shorter than $93 \mathrm{~nm}$, and an experimental measurement for longer wavelengths (Gibson et al. 1986). This non-absolute experiment includes the correct energy location of many resonances appearing in this cross section, including some that arise from excited fine-structure components of the $S$ ground state and may not be populated under all astrophysical conditions. The experiment was scaled to match the calculated cross section after integrating over the range 96 to $121 \mathrm{~nm}$.

\subsubsection{0. $\mathrm{Cl}$ - chlorine}

Two experimental $\mathrm{Cl}$ photoionisation cross sections are connected spanning from the $\mathrm{Cl}^{+}$ionisation threshold $(95.6 \mathrm{~nm})$ to $60 \mathrm{~nm}$. We adopt the measurement of Cantu \& Parkinson (1988) for wavelengths longer than $75 \mathrm{~nm}$, and Ruscic \& Berkowitz (1983) at shorter wavelengths.

\subsubsection{1. $\mathrm{K}$ - potassium}

The most recent experimental measurement of the potassium photoionisation cross section (Sandner et al. 1981) covers the wavelengths region between 238 and $285 \mathrm{~nm}$. We supplemented this between 120 and $238 \mathrm{~nm}$ with a recent R-matrix calculation (Zatsarinny \& Tayal 2010), and adopted a linearly-decreasing cross section at still shorter wavelengths.

\subsubsection{2. $\mathrm{Ca}$ - calcium}

A recent high-resolution measurement of the calcium photoabsorption cross section is measured by Yih et al. (1998) from $160 \mathrm{~nm}$ to the ionisation threshold at $202 \mathrm{~nm}$, and adopted here as a photoionisation cross section. This was combined with a further measurement that better resolves the two dominant resonant features at 176.5 and $188 \mathrm{~nm}$ (Carter et al. 1971), over the regions 176.3 to $176.8 \mathrm{~nm}$ and 187.1 to $189.6 \mathrm{~nm}$. The R-matrix calculation from the TOPbase database (Mendoza 1996) was used for wavelengths shorter than $160 \mathrm{~nm}$.
Table 2. References and wavelength ranges of concatenated Fe cross sections.

\begin{tabular}{ccl}
\hline \hline Wavelength $(\mathrm{nm})$ & Scaling $\left(\mathrm{cm}^{2}\right)^{a}$ & Reference \\
\hline$<80$ & - & Verner et al. (1996) \\
$80-88$ & - & Bautista (1997) \\
$88-123$ & $2.62 \times 10^{-18}$ & Tondello (1975) \\
$123-134$ & $1.88 \times 10^{-18}$ & Hansen et al. (1977) \\
$134-146$ & $2.01 \times 10^{-18}$ & Reed et al. (2009) \\
$146-158$ & $2.71 \times 10^{-18}$ & Reed et al. (2009) \\
\hline
\end{tabular}

Notes. ${ }^{(a)}$ The amount by which the arbitrary units of the various photoion yields were scaled to provide a cross section.

\subsubsection{Fe - iron}

Multiple measurements were combined into a single Fe photoionisation cross section file. An absolute cross section measured at $154 \mathrm{~nm}$ (Lombardi et al. 1978) was used to scale the wavelength-dependent relative photoionisation cross section of Hansen et al. (1977). In turn, this was used to calibrate the measurements of Reed et al. (2009) and Tondello (1975) by comparing integrated values over their overlapping ranges. For the case of Reed et al. (2009) separate calibration factors were used for the ranges 125 to $145 \mathrm{~nm}$ and 145 to $158 \mathrm{~nm}$ to account for an apparent wavelength dependence of laser power in their experiment. For wavelengths shorter than those covered by the various experiments the R-matrix calculation of Bautista (1997) was used, and at the shortest wavelengths the fitting formulate of Verner et al. (1996). The relevant scaling factors and wavelength ranges are listed in Table 2.

\subsubsection{4. $\mathrm{Zn}-$ zinc}

There are two measurements of the $\mathrm{Zn}$ photoabsorption cross section between $110 \mathrm{~nm}$ and the photoionisation threshold at 132 nm (Marr \& Austin 1969; Yih et al. 1998) that disagree by a factor of two. We used an average of these two measurements where they coincide, the measurement of Marr \& Austin (1969) between 91 and $110 \mathrm{~nm}$ and the relative-photoionisation cross section of Harrison et al. (1969) at shorter wavelengths, after scaling its integrated magnitude to match the continuum level of an R-matrix calculation (Huebner \& Mukherjee 2015).

\subsubsection{5. $\mathrm{H}_{2}$ - hydrogen}

Molecular hydrogen has a line-dominated spectrum beginning at $112 \mathrm{~nm}$ with absorption into the Lyman $\left(B^{1} \Sigma_{u}^{+} \leftarrow X^{1} \Sigma_{g}^{+}\right)$ and Werner $\left(C^{1} \Pi_{u} \leftarrow X^{1} \Sigma_{g}^{+}\right)$bands, and retains its resonant character even beyond the ionisation threshold at $80.3 \mathrm{~nm}$. The excited rovibrational lines and their tendency to partially predissociate or autoionise is well studied experimentally and theoretically by the group of Abgrall and Glass-Maujean et al. (Abgrall et al. 1993c,a,b, 1994; Abgrall \& Roueff 2006; Abgrall et al. 2000; Glass-Maujean et al. 2010, 2013a,b,c), culminating in a detailed experimental and theoretical database of absorption and emission lines ${ }^{14}$, as well as by other groups (e.g., Dehmer \& Chupka 1976; Chan et al. 1992; Samson \& Haddad 1994; Jonin et al. 2000; Philip et al. 2004; Hollenstein et al. 2006; Dickenson \& Ubachs 2014; Holland \& Shaw 2014).

Spontaneous emission from photoexcited $B^{1} \Sigma_{u}^{+}$and $C^{1} \Pi_{u}$ states into ground state vibrational levels produces a spectrum

\footnotetext{
14 molat. obspm. fr
} 
Table 3. $\mathrm{H}_{2}$ integrated photodissociation efficiency assuming several radiation field types.

\begin{tabular}{rc}
\hline \hline Radiation field type & Efficiency (fraction) \\
\hline ISRF & 0.12 \\
4000 K black body & 0.05 \\
10000 K black body & 0.11 \\
$20000 \mathrm{~K}$ black body & 0.13 \\
Solar & 0.25 \\
TW-Hydra & 0.16 \\
\hline
\end{tabular}

Notes. Assuming an ortho- $\mathrm{H}_{2}:$ para- $\mathrm{H}_{2}$ ratio of 0:1.

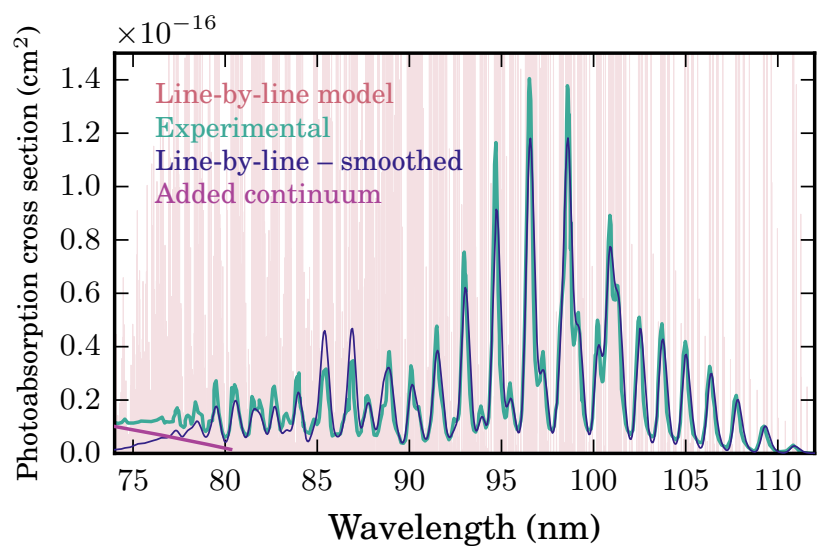

Fig. 15. $\mathrm{H}_{2}$ photoabsorption cross section. The under-resolved experimental measurement of (Chan et al. 1992), and a model spectrum before and after smoothing to the experimental resolution. Also shown is a correction to the model accounting for neglected excitation mechanisms.

of vacuum- and far-ultraviolet emission lines. Emission into the ground state continuum is also possible and provides a dissociation mechanism at wavelengths below the direct-photodissociation threshold of $84 \mathrm{~nm}$ (Field et al. 1966; Stecher \& Williams 1967; Abgrall et al. 1997, 1999). This spontaneous radiative dissociation mechanism is experimentally and theoretically verified (Dalgarno et al. 1970; Stephens \& Dalgarn 1972). The overall $\mathrm{H}_{2}$ dissociation efficiency assuming several of the radiation fields discussed in Sect. 2 is listed in Table 3 and varies between 5 and $15 \%$ for continuum interstellar radiation fields. This efficiency is somewhat larger (up to 25\%) for the solar and simulated TW-Hydra radiation fields because these include flux shorter than the Lyman limit at $91.2 \mathrm{~nm}$ and the dissociation fraction of the $\mathrm{H}_{2}$ cross section increases with decreasing wavelength.

Measured and calculated transition frequencies, strengths, and predissociation efficiencies of individual rovibrational transitions from the database of Abgrall and Glass-Maujean et al. were employed to reconstruct the temperature-dependent photoabsorption cross sections for $\mathrm{H}_{2}$ line-by-line, including excitation to $B^{1} \Sigma_{u}^{+}, B^{\prime}{ }^{1} \Sigma_{u}^{+}, C^{1} \Pi_{u}$, and $D^{1} \Pi_{u}$ excited valence states, and many $n p \pi$ and $n p \sigma$ Rydberg levels. The completeness of this database for wavelengths longer than $80 \mathrm{~nm}$ is evidenced by the agreement within $10 \%$ over this region of its integrated cross section with the low-resolution electron-energy-loss-derived absolute cross section of Chan et al. (1992). This comparison is shown in Fig. 15 after smoothing the line-by-line model spectrum to the under resolved experimental spectrum. The deficit of the model cross section relative to Chan et al. (1992) for wavelengths shorter than $80 \mathrm{~nm}$ was assumed to arise from direct
Table 4. $\mathrm{H}_{2}$ photodissociation rate in the interstellar radiation field of Draine (1978).

\begin{tabular}{cc}
\hline \hline & Rate $\left(\mathrm{s}^{-1}\right)$ \\
\hline Present result assuming ortho:para $=0: 1$ & $5.1 \times 10^{-11}$ \\
Present result assuming ortho:para $=3: 1$ & $5.7 \times 10^{-11}$ \\
Sternberg et al. (2014) & $5.8 \times 10^{-11}$ \\
\hline
\end{tabular}

ionisation to $\mathrm{H}_{2}^{+}$or autoionising highly-excited $\mathrm{H}_{2}$ levels that are neglected in the line-by-line model. This deficit is then added to the line-by-line model forming a continuum shortwards of $80 \mathrm{~nm}$. The cross section of Backx et al. (1976) was adopted at the shortest wavelengths, $<62 \mathrm{~nm}$. The use of a line-absorption model permits calculation of cross sections with a range of ground-state excitation states and Doppler profiles.

There is a great deal of previous work done on $\mathrm{H}_{2}$ photodissociation due to its importance to the balance of atomic and molecular hydrogen in the Universe. This subject is well reviewed and state-of-the-art calculations made in Sternberg et al. (2014) and references therein. The correctness of our simulated $\mathrm{H}_{2}$ photodissociation cross section is verified by comparing an ISRF dissociation rate with the calculations of Sternberg et al. (2014), listed in Table 4. Two calculations are made, for an ortho:para ratio of $\mathrm{H}_{2}$ ground state levels of $3: 1$ and $0: 1$. The increased rate for the $3: 1$ case is due to increased population of rotationally-excited $\mathrm{H}_{2}$ levels that are more likely to decay dissociatively.

\subsubsection{6. $\mathrm{H}_{3}^{+}$- trihydrogen cation}

The electronic excitation of cold $\mathrm{H}_{3}^{+}$has not been measured in the laboratory, due to the difficult of cooling the highly-symmetric radical ions that must be formed in situ, although its photodissociation from excited ground state rotational-vibrational levels is studied in some detail (e.g., Carrington \& Kennedy 1984). Calculations of its excited states find no allowed transitions with wavelength longer than about $70 \mathrm{~nm}$ (Talbi \& Saxon 1988), well above its $283 \mathrm{~nm}$ ground state dissociation threshold (Kulander \& Bottcher 1978). Equilibrium potential-energies and transition moments for the ground state accessible by photoabsorption are calculated by Talbi \& Saxon (1988) and van Dishoeck (unpublished), leading to very similar cross sections. The cross section of van Dishoeck was adopted for our photodissociation database.

\subsubsection{7. $\mathrm{CH}_{2}^{+}$- methylene cation}

According to the calculations of Theodorakopoulos \& Petsalakis (1991) this ion has many dipole-allowed excited states below $13.6 \mathrm{eV}$. The 1,2 and $3^{2} \mathrm{~B}_{2}, 2,3$ and $4{ }^{2} \mathrm{~A}_{1}$ and $2^{2} \mathrm{~B}_{1}$ states were included in our cross section with $f=0.008,0.0001,0.02,0.01$, $0.06,0.05$ and 0.03 , respectively, and $\eta^{\mathrm{d}}=1$ assumed.

\subsubsection{8. $\mathrm{CH}_{3}-$ methyl radical}

Several $\mathrm{CH}_{3}$ absorption bands are photographically observed between 120 and $230 \mathrm{~nm}$ by Herzberg \& Shoosmith (1956) revealing predissociation-broadened bands with widths of around $1 \mathrm{~nm}$, and very roughly estimated strengths. Higherresolution absolutely-calibrated photoabsorption measurements of the longest-wavelength absorption features (Cameron et al. 2002; Khamaganov et al. 2007; $200 \mathrm{~nm}$ to the photoabsorption 
A. N. Heays et al.: Photodissociation and photoionisation of atoms and molecules of astrophysical interest

Table 5. References and wavelength ranges of concatenated $\mathrm{CH}_{3}$ cross sections.

\begin{tabular}{cl}
\hline \hline Wavelength $(\mathrm{nm})$ & Reference \\
\hline $200-220$ & Cameron et al. (2002) \\
$126-200$ & Herzberg \& Shoosmith (1956) and as- \\
& sumed continuum \\
$108-126$ & Gans et al. (2010) \\
$<108$ & Extrapolation of Gans et al. (2010) \\
\hline
\end{tabular}

threshold at $220 \mathrm{~nm}$ ) allow for a rough calibration of the other photographic features based on their apparent saturation.

An absolute photoionisation cross section is recorded in the neighbourhood of the $126 \mathrm{~nm}$ ionisation threshold (Gans et al. 2010), which was extrapolated here to shorter wavelengths. A summary of cross sectional data sources is given in Table 5 .

\subsubsection{9. $\mathrm{CH}_{4}$ - methane}

$\mathrm{CH}_{4}$ photoabsorbs significantly at wavelengths shorter than $140 \mathrm{~nm}$ with an unstructured cross section that peaks at $90 \mathrm{~nm}$, indicating mostly direct photodissociation. Here, the experimental cross section of Kameta et al. (2002) between 52 and $124 \mathrm{~nm}$ was used, and the slightly-lower resolution data of Au et al. (1993) outside this range. The cross sections of Kameta et al. (2002) was scaled down by a factor or 0.95 in order to agree with other more reliably calibrated overlapping measurements (Au et al. 1993; Lee 2009).

Many neutral and ionised fragments are observed following $\mathrm{CH}_{4}$ photodissociation and these data are well summarised elsewhere (Backx \& Vanderwiel 1975; Huebner et al. 1992; Gans et al. 2011; Blitz \& Seakins 2012; Huebner \& Mukherjee 2015). Here, the wavelength-dependent branching ratios of Kameta et al. (2002) were used to decompose photoabsorption into ionisation and dissociation cross sections, assuming zero branching to fluorescence.

\subsubsection{0. $\mathrm{CH}_{4}^{+}-$methane cation}

This ion is subject to detailed theoretical studies indicating several dissociative excited states (van Dishoeck et al. 1980). Oscillator strengths into the $2,3{ }^{2} \mathrm{~A}_{1}$ and $2^{2} \mathrm{~B}_{1}$ states are taken to be $f=0.04,0.04$ and 0.08 , respectively, with $\eta^{\mathrm{d}}=1$.

\subsubsection{1. $\mathrm{C}_{2} \mathrm{H}$ - ethynyl radical}

Absorption into the $5^{2} \Sigma^{+}$state lying around $10 \mathrm{eV}$ dominates the interstellar photodissociation of this molecule. The higher ${ }^{2} \Pi$ states in the 8.5 to $10.5 \mathrm{eV}$ range can also contribute significantly as far as the $\mathrm{C}_{2} \mathrm{H}$ ionization potential of $11.4 \mathrm{eV}$. The oscillator strengths listed in Table 1 of the calculations of van Hemert \& van Dishoeck (2008) are used with $\eta^{\mathrm{d}}=1$.

\subsubsection{2. $\mathrm{C}_{2} \mathrm{H}_{2}$ - acetylene}

The $\mathrm{C}_{2} \mathrm{H}_{2}$ photoabsorption cross section is compiled from a collection of measurements (Wu et al. 1989; Xia et al. 1991; Cooper et al. 1995; Vattulainen et al. 1997; Cheng et al. 2011) over the wavelength ranges listed in Table 6 , which extend beyond $200 \mathrm{~nm}$ and are strongest shortward of $153 \mathrm{~nm}$. A strong absorption line of $\mathrm{C}_{2} \mathrm{H}_{2}$ coincides very nearly with the hydrogen Lyman- $\alpha$ line.
Table 6. References and wavelength ranges of concatenated $\mathrm{C}_{2} \mathrm{H}_{2}$ cross sections.

\begin{tabular}{cl}
\hline \hline Wavelength (nm) & Reference \\
\hline $6-106$ & Cooper et al. (1995) \\
$106-110$ & Xia et al. (1991) \\
$110-154$ & Cheng et al. (2011) \\
$154-210$ & Wu et al. (1989) \\
$210-300$ & Vattulainen et al. (1997) \\
\hline
\end{tabular}

The longest $\mathrm{C}_{2} \mathrm{H}_{2}$ photodissociation threshold, forming $\mathrm{C}_{2} \mathrm{H}+\mathrm{H}$, occurs at $217 \mathrm{~nm}$ and the dissociation efficiency at shorter wavelengths was studied several times (e.g., Okabe 1983; Seki \& Okabe 1993; Läuter et al. 2002; Kovács et al. 2010), as well as the probability of forming an $\mathrm{H}_{2}$ product. Läuter et al. (2002) convincingly determined a $100 \%$ efficiency for $\mathrm{H}$-atom formation by 121.6 and $193.3 \mathrm{~nm}$ radiation after detecting these atoms through laser-induced fluorescence, and a $100 \%$ dissociation efficiency was assumed for all wavelengths shorter than the $217 \mathrm{~nm}$ threshold.

\subsubsection{3. $\mathrm{C}_{2} \mathrm{H}_{6}$ - ethane}

The photoabsorption cross section of $\mathrm{C}_{2} \mathrm{H}_{6}$ is measured between 120 and $150 \mathrm{~nm}$ by Chen \& Wu (2004) and their $150 \mathrm{~K}$ measurement was adopted here (the temperature variation measured in this experiment was slight however). At shorter wavelengths, the cross section measured by Kameta et al. (1996) was used after scaling this down to match the overlapping region of Chen \& $\mathrm{Wu}$ (2004) (a factor of 0.83). The ionisation fraction for $\mathrm{C}_{2} \mathrm{H}_{6}$ is also measured by Kameta et al. (1996) and adopted here.

\subsubsection{4. $\mathrm{C}_{3}$ - tricarbon}

The photoabsorption cross section of $\mathrm{C}_{3}$ is difficult to measure due to its radical nature. Vertical excitation energies are calculated by van Hemert \& van Dishoeck (2008) for absorption into 5 highly-excited states as well as vertical oscillator strengths. We combine these transitions into a photodissociation cross section after assigning all states a Gaussian band profile of fullwidth half-maximum $18 \mathrm{~nm}$. This width was selected to match the width of the strong $\mathrm{C}_{3}$ electronic transition near $160 \mathrm{~nm}$ (corresponding to the $1^{1} \Sigma_{u}^{+}-1^{1} \Sigma_{g}^{+}$transition among the calculations of van Hemert \& van Dishoeck 2008) observed in a matrixisolation experiment (Monninger et al. 2002). Here, all bands were assumed to be $100 \%$ dissociative, since they lie well above the $\mathrm{C}_{3}$ dissociation limit.

There is no absolute measurement of the photoionisation cross section of $\mathrm{C}_{3}$ shorter than its $11.6 \mathrm{eV}$ threshold (Belau et al. 2007). The onset of ionisation and its wavelength dependence is measured by Nicolas et al. (2006). This wavelength dependence was adopted as a photoionisation cross section after scaling it to the typical molecular value of $2 \times 10^{-17} \mathrm{~cm}^{2}$ at $91.2 \mathrm{~nm}$.

\subsubsection{5. $\mathrm{I}-\mathrm{C}_{3} \mathrm{H}$ - linear propynylidyne}

The photodissociation of this linear molecule is dominated by the high-lying ${ }^{2} \Pi$ states around $7.8 \mathrm{eV}$. The $f$-values given in Table 3 of van Hemert \& van Dishoeck (2008) are to simulate the $l-\mathrm{C}_{3} \mathrm{H}$ spectrum, assuming $\eta^{\mathrm{d}}=1$. 


\subsubsection{6. $c-\mathrm{C}_{3} \mathrm{H}-$ cyclic propynylidyne}

In contrast with its linear counterpart, many different electronic states in the 5 to $7.5 \mathrm{eV}$ range can contribute to the photodissociation of cyclic $\mathrm{C}_{3} \mathrm{H}$. The $f$-values given in Table 4 of van Hemert \& van Dishoeck (2008) are used with $\eta^{\mathrm{d}}=1$.

\subsubsection{7. $\mathrm{HC}_{3} \mathrm{H}$ - propargylene}

Three isomers of $\mathrm{H}_{2} \mathrm{C}_{3}$ are treated in the calculations of van Hemert \& van Dishoeck (2008). In this section, $\mathrm{HC}_{3} \mathrm{H}$ refers to the $\mathrm{HCCCH}$ linear form, with the alternative linear excited isomer $\left(\mathrm{H}_{2} \mathrm{CCC}\right.$ or $\left.l-\mathrm{C}_{3} \mathrm{H}_{2}\right)$ and cyclic ground isomer $\left(c-\mathrm{C}_{3} \mathrm{H}_{2}\right)$ discussed below. The photodissociation of $\mathrm{HC}_{3} \mathrm{H}$ is dominated by three excited ${ }^{3} \mathrm{~A}$ levels, with vertical excitations of 4.23, 6.29, and $7.52 \mathrm{eV}$ above the ground state.

\subsubsection{8. $\mathrm{I}-\mathrm{C}_{3} \mathrm{H}_{2}$ - linear propenylidene}

The strongest absorptions of this molecule are into the higher ${ }^{1} A_{1}$ Rydberg states around $9 \mathrm{eV}$, below the ionization potential at $10.4 \mathrm{eV}$. The $f$-values given in Table 7 of van Hemert \& van Dishoeck (2008) are used, with $\eta^{\mathrm{d}}=1$. The low-lying $\tilde{C}(2)^{1} A_{1}$ state is taken into account, even though it is not clear how efficient predissociation is for this state. The linear $\mathrm{HC}_{3} \mathrm{H}$ isomer has a very different electronic structure from $l-\mathrm{C}_{3} \mathrm{H}_{2}$ with a triplet ground state. This molecule is included as a separate species in the database, following Table 5 of van Hemert \& van Dishoeck (2008).

\subsubsection{9. $c-\mathrm{C}_{3} \mathrm{H}_{2}-$ cyclic propenylidene}

This molecule has only a few low-lying dipole-allowed electronic states. The largest oscillator strengths are found to transitions in the 9 to $11 \mathrm{eV}$ range, which is above the ionization potential of $c-\mathrm{C}_{3} \mathrm{H}_{2}$ at $9.15 \mathrm{eV}$. The current version of the database, based on Table 6 of van Hemert \& van Dishoeck (2008) with $\eta^{\mathrm{d}}=1$, assumes that these transitions lead to ionization rather than dissociation. If they would lead to dissociation, the interstellar photodissociation rate would be increased by a factor of 2 .

\subsubsection{0. $1-\mathrm{C}_{4}$ - linear tetracarbon}

The strongest absorption occurs into the $2{ }^{3} \Sigma_{u}^{-}$state around $6.95 \mathrm{eV}$, which, like a similar state for $\mathrm{C}_{3}$, has a huge oscillator strength of 1.6. This state, together with other states listed in Table 8 of van Hemert \& van Dishoeck (2008), are included with $\eta^{\mathrm{d}}=1$. Rhombic $\mathrm{C}_{4}$, which is almost isoenergetic with $\ell$ $\mathrm{C}_{4}$, is not considered and may well have a significantly different photodissociation rate.

\subsubsection{1. $\mathrm{I}-\mathrm{C}_{4} \mathrm{H}$ - butadiynyl}

Of the dipole-allowed transitions below the ionization potential at $9.6 \mathrm{eV}$, the higher ${ }^{2} \Sigma^{+}$states at 7 to $9 \mathrm{eV}$ have orders of magnitude larger oscillator strengths than other states and thus dominate the interstellar photodissociation. The values used in Table 9 of van Hemert \& van Dishoeck (2008) are used with $\eta^{\mathrm{d}}=1$.
Table 7. References and wavelength ranges of concatenated $\mathrm{H}_{2} \mathrm{O}$ cross sections.

\begin{tabular}{|c|c|c|}
\hline Wavelength (nm) & Scaling & Reference \\
\hline $6.2-56.6$ & 1 & Chan et al. (1993b) \\
\hline $56.6-98.95$ & 1 & Fillion et al. (2003) \\
\hline $98.95-100$ & 1 & Linear interpolation $^{a}$ \\
\hline $100-107.28$ & 1 & Fillion et al. (2004) \\
\hline $107.28-108.01$ & $0.5^{b}$ & CfA molecular database ${ }^{c}$ \\
\hline $108.01-111.24$ & 1 & Fillion et al. (2004) \\
\hline $111.24-111.77$ & $0.5^{b}$ & CfA molecular database ${ }^{c}$ \\
\hline $111.77-113.9$ & 1 & Fillion et al. (2004) \\
\hline $113.9-114.8$ & 1 & Linear interpolation $^{a}$ \\
\hline $114.8-123.42$ & $1.09^{d}$ & Mota et al. (2005) \\
\hline $123.42-124.5$ & $0.91^{e}$ & CfA molecular database $e^{c, f}$ \\
\hline $124.5-193.9$ & $1.09^{d}$ & Mota et al. (2005) \\
\hline
\end{tabular}

Notes. ${ }^{(a)}$ Linearly interpolated between surrounding cross section data. (b) Scaled to match the integrated overlapping cross section of Fillion et al. (2004). (c) www.cfa.harvard.edu/amp/ampdata/ cfamols.html. ${ }^{(d)}$ Scaled to match the integrated overlapping cross section of Chan et al. (1993b). ${ }^{(e)}$ Scaled to match the integrated overlapping cross section of Mota et al. (2005). ${ }^{(f)}$ An apparent error in the wavelength calibration of this spectrum was corrected by adjusting the spectrum longwards by $0.05 \mathrm{~nm}$.

\subsubsection{2. $\mathrm{I}-\mathrm{C}_{5} \mathrm{H}$ - pentynylidyne}

The higher ${ }^{2} \Pi$ states in the 4 to $6 \mathrm{eV}$ range dominate the photodissociation of this molecule, using the values in Table 10 of van Hemert \& van Dishoeck (2008) with $\eta^{\mathrm{d}}=1$.

\subsubsection{3. $\mathrm{H}_{2} \mathrm{O}$ - water}

Measurements of the $\mathrm{H}_{2} \mathrm{O}$ photoabsorption cross section as a function of wavelength date back many decades (see van Dishoeck et al. (2013, Sect. 3.1.3)). Several measurements were concatenated into a single cross section file, as detailed in Table 7. Some of these data were rescaled to agree with the integrated cross section of more-reliable absolute measurements.

An absolutely-calibrated low-resolution cross section measured by electron-energy loss spectroscopy (Chan et al. 1993b), including the entire ultraviolet range from its onset at $190 \mathrm{~nm}$, was combined with several higher-resolution direct photoabsorption measurements covering various portions of the spectrum (Smith et al. 1981; Yoshino et al. 1996, 1997; Parkinson \& Yoshino 2003; Fillion et al. 2003, 2004; Mota et al. 2005 , and others). Some of these resolve temperature-dependent rotational structure. Fillion et al. (2003) record the photoionisation yield of $\mathrm{H}_{2} \mathrm{O}$ that was used to partition the experimental photoabsorption cross section between ionisation and dissociation.

The large abundance of water observed in interstellar space (van Dishoeck et al. 2013) means that its photodissociation products, either $\mathrm{O}$ or $\mathrm{OH}$, are significant participants in the ongoing chemistry. A special case was made of further dividing the water photodissociation cross section into cross sections producing $\mathrm{O}$ or $\mathrm{OH}$ radicals. The product branching ratios are accurately measured at the Lyman- $\alpha$ wavelength (Slanger \& Black 1982; Mordaunt et al. 1994) and some information is available at other wavelengths (Ung 1974; Stief et al. 1975). A coupledstates quantum mechanical wavepacket simulation of $\mathrm{H}_{2} \mathrm{O}$ photodissociation is carried out by van Harrevelt \& van Hemert (2008) on multidimensional potential-energy surfaces describing its ground state and two excited states. This calculation 
A. N. Heays et al.: Photodissociation and photoionisation of atoms and molecules of astrophysical interest

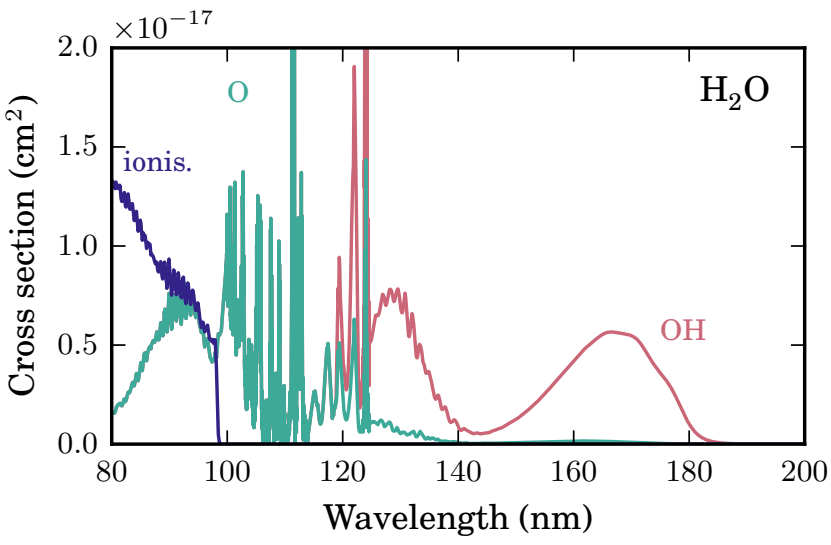

Fig. 16. Photoabsorption cross section of $\mathrm{H}_{2} \mathrm{O}$. Divided into channels producing $\mathrm{O}$ (green), $\mathrm{OH}$ (red), and ionisation (blue).

Table 8. References and wavelength ranges of concatenated $\mathrm{O}_{2}$ cross sections.

\begin{tabular}{cl}
\hline \hline Wavelength (nm) & Reference \\
\hline $5-49$ & Matsunaga \& Watanabe (1967), MPI \\
$49-108$ & Mainz UV/Vis database \\
$108-115$ & Holland et al. (1993) \\
$115-179$ & Luawa \& Ogawa (1975) \\
$179-203$ & Yoshino et al. (1992) \\
$205-240$ & Yoshino et al. (1988) \\
\hline
\end{tabular}

agrees with the experimental $\mathrm{O}$ and $\mathrm{OH}$ branching ratios at $121.6 \mathrm{~nm}$ within $10 \%$ and the calculated wavelength dependence between 118 and $146 \mathrm{~nm}$ was adopted here. This ratio was linearly extrapolated to zero at the threshold O-atom production, $177 \mathrm{~nm}$ (Mordaunt et al. 1994). For wavelengths shorter than $118 \mathrm{~nm}$, an equal production of $\mathrm{OH}$ and $\mathrm{H}$ radicals was assumed. The resulting partial cross sections are plotted in Fig. 16. van Harrevelt \& van Hemert (2008) also calculated significant branching between the pair of products and excitations states $\mathrm{O}\left({ }^{3} \mathrm{P}\right)+2 \mathrm{H}$ and $\mathrm{O}\left({ }^{3} \mathrm{D}\right)+\mathrm{H}_{2}$, as well as $\mathrm{OH}\left(\mathrm{X}^{2} \Pi\right)+\mathrm{H}$ and $\mathrm{OH}\left(\mathrm{X}^{2} \Sigma\right)+\mathrm{H}$.

\subsubsection{4. $\mathrm{H}_{2} \mathrm{O}^{+}$- water ion}

This molecule is remarkably transparent at ultraviolet wavelengths: calculations show that there are no dipole-allowed dissociative electronic states below $13.6 \mathrm{eV}$ so that the interstellar $\mathrm{H}_{2} \mathrm{O}^{+}$photodissociation rate is negligible (van Dishoeck et al. 2006).

\subsubsection{5. $\mathrm{O}_{2}$ - oxygen}

There are many laboratory studies of $\mathrm{O}_{2}$ photoabsorption. The cross section adopted here is compiled from several sources listed in Table 8. The photoionisation efficiency of Holland et al. (1993) was used to partition the photoabsorption cross section between dissociative and ionising branches shortwards of the ionisation threshold at $103 \mathrm{~nm}$.

\subsubsection{6. $\mathrm{O}_{2}^{+}$- oxygen ion}

This molecule has no dissociative electronic states below $13.6 \mathrm{eV}$ (Honjou et al. 1978). The only dipole-allowed states
Table 9. References and wavelength ranges of concatenated $\mathrm{H}_{2} \mathrm{O}_{2}$ cross sections.

\begin{tabular}{cl}
\hline \hline Wavelength (nm) & Reference \\
\hline $65-105$ & Litorja \& Ruscic (1998) \\
$105-195$ & Suto \& Lee (1983) \\
$195-350$ & Lin et al. (1978) \\
\hline
\end{tabular}

Notes. ${ }^{(a)}$ Scaled to agree with the absolutely-calibrated photoionisation cross section of Dodson et al. (2015) where they overlap.

Table 10. References and wavelength ranges of concatenated $\mathrm{O}_{3}$ cross sections.

\begin{tabular}{cl}
\hline \hline Wavelength $(\mathrm{nm})$ & Reference \\
\hline $53-61$ & Ogawa \& Cook (1958) \\
$61-93$ & Berkowitz (2008) \\
$93-110$ & Ogawa \& Cook (1958) \\
$110-173$ & Mason et al. (1996) \\
$173-212$ & Ackerman (1971) \\
$212-1100$ & Serdyuchenko et al. (2014) \\
\hline
\end{tabular}

below $13.6 \mathrm{eV}$ and above the $\mathrm{O}_{2}^{+}$dissociation limit are the $\mathrm{A}^{2} \Pi_{u}$ and $2^{2} \Pi_{u}$ states. Their oscillator strengths are only $f=0.005$ each and, since they are bound, $\eta^{\mathrm{d}}=0$ is assumed.

\subsubsection{7. $\mathrm{H}_{2} \mathrm{O}_{2}$ - hydrogen peroxide}

The combined experimental photoabsorption cross sections of Lin et al. (1978) and Suto \& Lee (1983) extend from 105 to $195 \mathrm{~nm}$, with the absorption at the longest wavelength being very small (less than $10^{-21} \mathrm{~cm}^{2}$ ) and likely to be temperature dependent. A photoionisation cross section was assembled by combining absolute and relative measurements of $\mathrm{H}_{2} \mathrm{O}_{2}^{+}$production (Dodson et al. 2015; Litorja \& Ruscic 1998) and assuming a low production of fragmented ions.

\subsubsection{8. $\mathrm{O}_{3}$ - ozone}

The $\mathrm{O}_{3}$ photoabsorption cross section is compiled from a selection of many measurements and includes all wavelengths between 53 and $1100 \mathrm{~nm}$, with the cross section longwards of $325 \mathrm{~nm}$ never exceeding $1 \times 10^{-20} \mathrm{~cm}^{-2}$. The references and ranges of concatenated cross sections are give in Table 10. The binding energy of the $\mathrm{O}_{3}$ ground state corresponds to a wavelength of $1180 \mathrm{~nm}$ and all excited states decay dissociatively, even those absorbing weakly in the near-infrared (Grebenshchikov et al. 2007). The photoionisation threshold is $99 \mathrm{~nm}$ and a total photoionisation cross sections is generated by Berkowitz (2008) from reanalysis of the photoionisation yields of $\mathrm{O}_{3}^{+}, \mathrm{O}_{2}^{+}$, and $\mathrm{O}^{+}$ions measured by Mocellin et al. (2001).

\subsubsection{9. $\mathrm{CO}$ - carbon monoxide}

The photodissociation of the strongly bound $\mathrm{CO}$ molecule starts shortward of $110 \mathrm{~nm}$ and occurs exclusively by predissociation. A detailed model of the completely line-dominated $\mathrm{CO}$ photodissociation cross section is constructed by Visser et al. (2009) based on wavelengths, oscillator strengths, and predissociation probabilities for individual lines and from a variety of experimental sources (e.g., Eidelsberg \& Rostas 1990; Ubachs et al. 1994; Eidelsberg et al. 2006; Cacciani et al. 2001). Synthetic 
Table 11. References and wavelength ranges of concatenated photoabsorption $\mathrm{H}_{2} \mathrm{CO}$ cross sections.

\begin{tabular}{cl}
\hline \hline Wavelength (nm) & Reference \\
\hline $6-60$ & Cooper et al. (1996) \\
$60-176$ & Mentall et al. (1971) \\
$220-375$ & Meller \& Moortgat (2000) \\
\hline
\end{tabular}

Notes. ${ }^{(a)}$ Published absorption coefficient scaled by $3.9 \times 10^{-20}$ to agree with the absolutely-calibrated cross section of Suto et al. (1986) where they overlap.

spectra were then constructed by summing the profile of all lines and including the effects of predissociation broadening, Doppler broadening, and alternative excitation temperatures. Visser et al. considered all of the important isotopologues in their analysis and proceeded to detailed calculations of photodissociation rates and shielding-functions, estimating the uncertainty of their cross sections to be $20 \%$.

For the line-dominated part of the $\mathrm{CO}$ spectrum between 91 and $110 \mathrm{~nm}$, a simulated photoabsorption and dissociation cross sections was constructed from the line parameters of Visser et al. This spectrum is shown in Fig. 8 assuming an excitation temperature of $100 \mathrm{~K}$ and a Doppler broadening of $1 \mathrm{~km} \mathrm{~s}^{-1}$. These values are also used in the various calculations involving $\mathrm{CO}$ later in the paper. The shorter wavelength part of the spectrum, $<91 \mathrm{~nm}$, is taken from the electron-energy loss measurement of Chan et al. (1993a) that does not resolve the CO band structure between 80 and $91.2 \mathrm{~nm}$. The non-dissociative part of the spectrum longer than $108 \mathrm{~nm}$ is also taken from the measurement of Chan et al. (1993a) and does not include the full rotational structure of these bands.

\subsubsection{0. $\mathrm{CO}^{+}-$carbon monoxide ion}

Like $\mathrm{CO}, \mathrm{CO}^{+}$has a deep potential well with a dissociation energy of $8.34 \mathrm{eV}$. The higher excited $\mathrm{D}^{2} \Pi, \mathrm{G}, \mathrm{E}$ and $\mathrm{F}^{2} \Sigma^{+}$ states are likely to be (pre-)dissociated, however, with oscillator strengths to the $\mathrm{D}$ and $\mathrm{G}$ states computed to be $f=0.01$ and 0.02 , respectively (Lavendy et al. 1993). Similar values were assumed for the higher states, with $\eta^{\mathrm{d}}=1$.

\subsubsection{1. $\mathrm{CO}_{2}$ - carbon dioxide}

$\mathrm{CO}_{2}$ is an important atmospheric molecule and thus well studied. A compilation representing its photodissociation cross section is made by Huestis \& Berkowitz (2010), and was used here after being updated between 87 and $109 \mathrm{~nm}$ with a more recent higher-resolution photoabsorption cross section (Archer et al. 2013). The photoionisation cross section of (Shaw et al. 1995) was adopted.

\subsubsection{2. $\mathrm{HCO}^{+}-$isoformyl}

The photodissociation of this ion is studied in detail theoretically (Koch et al. 1995a,b). The only dipole-allowed dissociative state is the $1^{1} \Pi$ state around $11.5 \mathrm{eV}$ with a small cross section.

\subsubsection{3. $\mathrm{H}_{2} \mathrm{CO}$ - formaldehyde}

The electron-energy loss derived cross section of Cooper et al. (1996) was combined with the VUV photoabsorption spectra of
Mentall et al. (1971) and Meller \& Moortgat (2000), as listed in Table 11. The weak UV absorption between 240 and $360 \mathrm{~nm}$ is just shortwards of the effective threshold resulting from an internal barrier for dissociation to $\mathrm{H}_{2}$ and $\mathrm{CO}$ (Hopkins et al. 2007).

The highly-structured $\mathrm{H}_{2} \mathrm{CO}$ spectrum between 115 and $160 \mathrm{~nm}$ consists of predissociated Rydberg series, including some showing rotational structure (Brint et al. 1985), and presents the risk of cross sections deduced from underresolved photoabsorption spectra being underestimated. However, the integrated cross section of an absolutely-calibrated medium-resolution photoabsorption cross section (Suto et al. 1986) agrees with the resolution-independent electron-energy loss measurement (Cooper et al. 1996) within 4\%, negating this possibility. The 225 to $360 \mathrm{~nm}$ photodissociation cross section is also under resolved, but high-resolution data (e.g., Pope et al. 2005; Ernest et al. 2012) will not affect $\mathrm{H}_{2}$ CO's astrophysical photodestruction properties.

The photoionisation cross section is measured near threshold by Dodson et al. (2015) and at shorter wavelengths the photoabsorption cross section was scaled by the ionisation efficiency of Cooper et al. (1996). This ionisation efficiency approaches $100 \%$ at longer wavelengths than that measured by Mentall et al. (1971), which suffers from an estimated $30 \%$ uncertainty.

\subsubsection{4. $\mathrm{NH}$ - imidogen}

Experimental data on photoabsorption cross sections for NH exist only for the lower nondissociative excited states (Krishnamurty \& Narasimham 1969) and via two-photon transitions (de Beer et al. 1991; Clement et al. 1992). The direct dissociation of $\mathrm{NH}$ into its first two excited repulsive curves is calculated by Kirby \& Goldfield (1991) from ab initio potentialenergy curves and electronic transition moments, and we adopt their theoretical cross sections. An additional broad feature at $100 \mathrm{~nm}$ with integrated cross section $10^{-16} \mathrm{~cm}^{2} \mathrm{~nm}$ was added to this cross section to roughly account for photoabsorption and dissociation into electronic states of higher energy than those calculated.

Predissociation of the $v=0,1$, and 2 bound levels of the low-lying $A^{3} \Pi$ state contributes negligibly to the NH photodissociation rate in an ISRF (Kirby \& Goldfield 1991), but are at sufficiently long wavelengths, greater than $250 \mathrm{~nm}$, that they may influence this rate in cool radiation fields. These three bound levels were included int our cross section database without simulating their rotational structure by adopting the wavelengths of (Huber \& Herzberg 1979), band oscillator strengths (the recommended values calculated by Kirby \& Goldfield 1991), and predissociation efficiencies. The latter are assumed to be 0,0 , and 0.5 for the $v=0,1$, and 2 levels, respectively, in line with their ratios of radiative and dissociative lifetimes deduced by Patel-Misra et al. (1991), and assuming rotational excitation is limited to below $J=10$, appropriate for astrophysical environments. The combined uncertainty in the excitation and dissociation of the $A^{3} \Pi v=2$ level is an order of magnitude. The addition of this level increases the photodissociation rate of $\mathrm{NH}$ in a $4000 \mathrm{~K}$ black body radiation field by $20 \%$. This contribution is less than the uncertainties associated with shorter-wavelength stronger-absorbing states.

A theoretical photoionisation cross section was taken from Wang et al. (1990) that has significant magnitude, $7.4 \times$ $10^{-18} \mathrm{~cm}^{2}$, at the Lyman- $\alpha$ wavelength. 
Table 12. References and wavelength ranges of concatenated $\mathrm{NH}_{3}$ cross sections.

\begin{tabular}{cl}
\hline \hline Wavelength (nm) & Reference \\
\hline $8-106$ & Samson et al. (1987) \\
$106-140$ & Wu et al. (2007) \\
$140-226$ & Liang et al. (2007) \\
\hline
\end{tabular}

Notes. ${ }^{(a)}$ To reduce scatter due to measurement noise this cross section was down-sampled between 106 and $115 \mathrm{~nm}$ into $0.16 \mathrm{~nm}$ intervals.

\subsubsection{5. $\mathrm{NH}_{2}$ - amidogen}

There are two ab initio calculations of the dissociative excited states, transition moments, and photodissociation cross sections of this radical. Here, a photodissociation cross section file was composed, from data for the very weak $1^{2} A^{\prime \prime} \rightarrow 2^{2} A^{\prime}$ transition between 150 and $210 \mathrm{~nm}$ (Saxon et al. 1983) added to the shorter-wavelength cross sections due to stronger transitions to various excited states calculated by Koch (1997) based on potential energy surfaces calculated by Vetter et al. (1996). An additional $2 \mathrm{~nm}$ FWHM Gaussian line of integrated cross section $10^{-16} \mathrm{~cm}^{2} \mathrm{~nm}$ was added to the photodissociation cross section at $110 \mathrm{~nm}$ to account for excitation into states not considered in the two calculations.

A photoionisation yield of $\mathrm{NH}_{2}$ is recorded by Gibson et al. (1985) from the ionisation threshold at $111 \mathrm{~nm}$. We arbitrarily adopt a photoionisation cross section of $2 \times 10^{-17} \mathrm{~cm}^{2}$ at $80 \mathrm{~nm}$, in line with other molecules in the database, in order to place this onto an absolute scale.

\subsubsection{6. $\mathrm{NH}_{3}$ - ammonia}

The results of two high-resolution optical experiments (Wu et al. 2007; Liang et al. 2007) were supplemented by an electron energy-loss measurement (Samson et al. 1987) over the wavelength ranges listed in Table 12 to determine a best photoabsorption cross section for $\mathrm{NH}_{3}$. Since the cross section is broad and continuous, $100 \%$ dissociation efficiency is assumed below the ionisation threshold.

Two measurements of the $\mathrm{NH}_{3}$ ionisation efficiency were used to divide the cross section between photoionisation and photoabsorption above the ionisation limit, Samson et al. (1987) between 8 and $105 \mathrm{~nm}$ and Xia et al. (1991) between 106 and $124 \mathrm{~nm}$. Samson et al. (1987) also determined the branching ratios of various dissociative photoionisation products with $\mathrm{NH}_{3}^{+}$ being the only ion product for wavelengths longer than $79 \mathrm{~nm}$.

$\mathrm{NH}_{3}$ is an abundant species in many astrophysical environments (e.g., Ho \& Townes 1983; Choi et al. 2010; Shinnaka et al. 2011), and the product branching of its dissociation may have a significant affect on the total abundance of astrochemical molecules $\mathrm{NH}_{2}$ and $\mathrm{NH}$. As for $\mathrm{H}_{2} \mathrm{O}$, a special case was made of determining the wavelength dependence of this branching.

A measurement of $\mathrm{NH}_{3}$ photolysis at $121.6 \mathrm{~nm}$ was made by Slanger \& Black (1982), and previous experiments reviewed therein, finding a 1.89 quantum yield of $\mathrm{H}$-atom production. This requires the formation of $\mathrm{NH}+\mathrm{H}+\mathrm{H}$ fragments with a branching ratio of $95 \%$ and the remaining $5 \%$ of photoabsorption events was assumed to produce $\mathrm{NH}_{2}+\mathrm{H}$. No further constraining information on these dissociation channels exists at shorter wavelengths and so the same 95 and 5\% branching was adopted. At longer wavelengths, the observed threshold for NH fluorescence

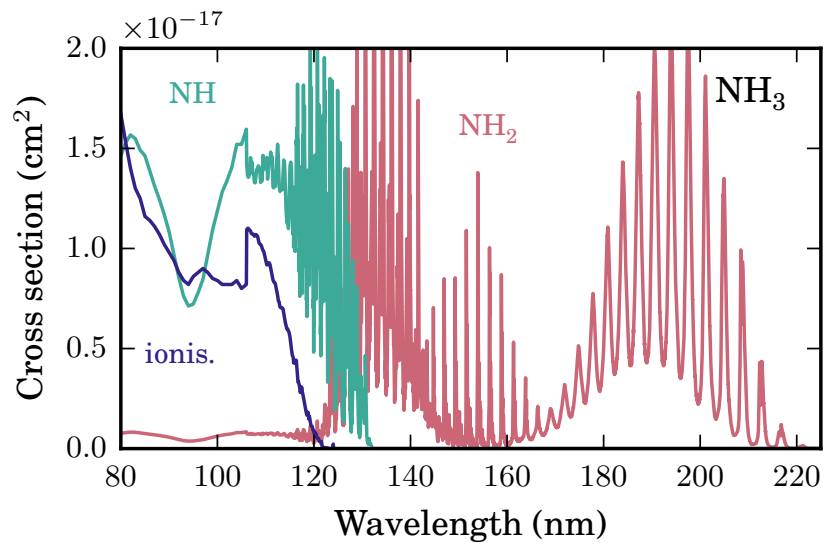

Fig. 17. Photoabsorption cross section of $\mathrm{NH}_{3}$. Divided into channels producing $\mathrm{NH}$ (green), $\mathrm{NH}_{2}$ (red), and ionisation (blue).

following $\mathrm{NH}_{3}$ dissociation is $132 \mathrm{~nm}$ (Leach et al. 2005), and $100 \% \mathrm{NH}_{2}$ production was assumed for all longer wavelengths. The branching ratios between 121.6 and $132 \mathrm{~nm}$ were linearly interpolate between experimental constraints. The resulting partial cross sections are plotted in Fig. 17.

\subsubsection{7. $\mathrm{N}_{2}$ - nitrogen}

Detailed studies of the astrophysical photodissociation of $\mathrm{N}_{2}$ and its isotopic consequences can be found in $\mathrm{Li}$ et al. (2013) and Heays et al. (2014b), and are based on a large body of work, both experimental (e.g., Carroll \& Collins 1969; Sprengers et al. 2003; Stark et al. 2008; Lewis et al. 2008; Heays et al. 2014a) and theoretical (e.g., Dressler 1969; Stahel et al. 1983; Lewis et al. 2005; Heays 2011).

The photoabsorption and dissociation cross sections of Heays et al. (2014b) between 85 and $100 \mathrm{~nm}$ were used. These are the product of a coupled-channels model (e.g., Gibson \& Lewis 1996) defined by excited-state potential-energy curves that are experimentally-optimised (Heays et al. 2011). These cross sections reproduce the full ro-vibrationally resolved spectrum of the molecule, including its temperature dependence, and have an uncertainty of $10 \%$ over the wavelength relevant to the ISRF. The simulation used here assumes a thermal excitation of $100 \mathrm{~K}$ and a Doppler broadening of $1 \mathrm{~km} \mathrm{~s}^{-1}$.

$\mathrm{N}_{2}$ does not absorb significantly at longer wavelengths than $100 \mathrm{~nm}$. Some highly-excited Rydberg states appearing at wavelengths shorter than $85 \mathrm{~nm}$ are missing in the coupled-channels formulation. The low-resolution electron-energy-loss-deduced cross sections of Chan et al. (1993d) and Shaw et al. (1992) were used over the wavelength ranges $79.5<\lambda<85$ and $\lambda<79.5 \mathrm{~nm}$, respectively. The cross section of Chan et al. (1993d) was scaled down by a factor of 0.7 in order to maintain continuity with the adjoining measurement.

\subsubsection{NO - nitric oxide}

The NO photoabsorption spectrum is well measured by lowresolution electron-energy-loss spectroscopy (Iida et al. 1986; Chan et al. 1993c) and at higher resolution by photoabsorption spectroscopy utilising $\mathrm{He}$ and $\mathrm{H}_{2}$ discharge sources to generate ultraviolet continua (Watanabe et al. 1967).

A further series of Fourier-transform spectroscopy measurements (Yoshino et al. 2006, and references therein) principally employing synchrotron radiation resolved many absorption lines 
Table 13. Assumed dissociation fraction of NO excited-state vibrational levels.

\begin{tabular}{|c|c|c|}
\hline Level & Fraction & Reference \\
\hline$A(3)^{a}$ & 0 & Brzozowski et al. (1976) \\
\hline$B(6)$ & 0 & Brzozowski et al. (1976) \\
\hline$B(7)^{b}$ & 0.05 & Brzozowski et al. (1976) \\
\hline$B(9)$ & 0.98 & Hikida \& Mori (1978) \\
\hline$B(10)^{c}$ & 1 & \multirow{9}{*}{ Brzozowski et al. (1976) } \\
\hline$B(11)^{c}$ & 1 & \\
\hline$B(12)^{c}$ & 1 & \\
\hline$B(14)^{c}$ & 1 & \\
\hline \multirow{2}{*}{$C(0)$} & $\begin{cases}0 & J<4.5\end{cases}$ & \\
\hline & $0.9 \quad J \geq 4.5$ & \\
\hline$C(1)^{d}$ & 1 & \\
\hline$C(2)^{d}$ & 1 & \\
\hline$C(3)^{d}$ & 1 & \\
\hline$D(0)$ & $\left(1+\frac{6000}{J(J+1)}\right)^{-1}$ & Luque \& Crosley (2000) \\
\hline$D(1)$ & $\left(1+\frac{460}{J(J+1)}\right)^{-1}$ & Luque \& Crosley (2000) \\
\hline$D(2)$ & $\left(1+\frac{290}{J(J+1)}\right)^{-1}$ & Luque \& Crosley (2000) \\
\hline$D(3)$ & $\left(1+\frac{120}{J(J+1)}\right)^{-1}$ & Luque \& Crosley (2000) \\
\hline
\end{tabular}

Notes. Level notation as in Yoshino et al. (2006). Rotational angularmomentum quantum number: $J{ }^{\left({ }^{a}\right)}$ Dissociates for rotational levels with $J>26$, (Brzozowski et al. 1976; Luque \& Crosley 2000) but this requires higher temperatures than are relevant here. ${ }^{(b)}$ This fraction is actually an upper limit (Brzozowski et al. 1974, 1976). ${ }^{(c)}$ No measurements exist but it is reasonable to expect these levels to dissociate completely because of the large dissociation fraction of the lower-energy level $B(9) .{ }^{(d)}$ Do not appear in emission (Brzozowski et al. 1976).

between 166 and $196 \mathrm{~nm}$, and reduced them to a list of wavelengths and oscillator strengths. These data were used to simulate this part of the spectrum with a line-by-line model capable of reproducing a range of excitation temperatures. As illustrated by various experiments, not all lines in this range dissociate completely (e.g., Brzozowski et al. 1976).

The predissociation fraction of the various excited levels was estimated from several studies (Brzozowski et al. 1974, 1976; Hikida \& Mori 1978; Hart \& Hepburn 1987; Luque \& Crosley 2000) and the assumed values are listed in Table 13 for excited levels appearing in our line-by-line simulation. Some of the lowest-excited rotational transitions were not observed in the room temperature spectrum of Yoshino et al. (2006) but are important in the interstellar medium. The various line parameters for these transitions were extrapolated from higher levels.

The fraction of NO photoabsorption below the $134 \mathrm{~nm}$ threshold resulting in ionisation was estimated from the photoion spectroscopy of Watanabe et al. (1967). The cross section plotted in Fig. 10 and used later in various calculations is simulated assuming a $100 \mathrm{~K}$ ground state excitation and $1 \mathrm{~km} \mathrm{~s}^{-1}$ Doppler width.

\subsubsection{9. $\mathrm{CN}$ - cyanide radical}

The photodissociating states of $\mathrm{CN}$ have not been experimentally observed. Ab initio configuration-interaction calculations (Lavendy et al. 1984, 1987) have identified high-lying valence states (the fourth and fifth members of ${ }^{2} \Pi$ and ${ }^{2} \Sigma^{+}$symmetry) to be responsible for its ultraviolet photodissociation cross section, and calculated its onset to occur near $105 \mathrm{~nm}$. A further

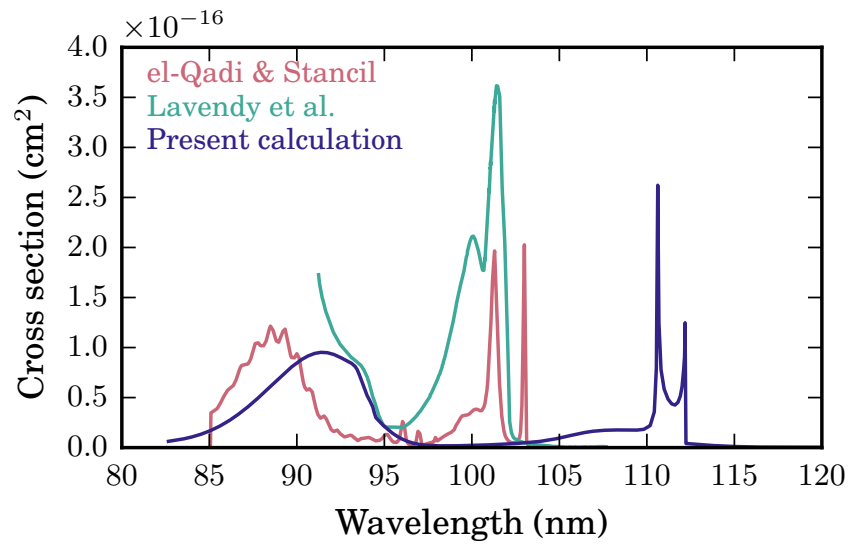

Fig. 18. Photodissociation cross section for CN. Presently calculated cross section and those of Lavendy et al. (1987) and el-Qadi \& Stancil (2013).

cross section is calculated by el-Qadi \& Stancil (2013) adopting the potential-energy curves and transition moments published by Lavendy et al. (1987) but finds an approximately factor-of-three discrepancy compared with the earlier work, as shown in Fig. 18. To resolve this, we recomputed the potential-energy curves of ${ }^{2} \Sigma^{+}$and ${ }^{2} \Pi$ states and their transition moments with respect to photoexcitation from the ground state. This analysis included all states dissociating to form excited $\mathrm{N}\left({ }^{4} \mathrm{~S}\right)$ atoms and ground state $\mathrm{C}\left({ }^{3} \mathrm{P}\right)$, or to lower energy limits, and some states leading to more highly-excited atoms.

Full details of the ab initio calculation using the MOLPRO software will be given in a later publication but adopts similar methods as other recent calculations of $\mathrm{CN}$ excited states (Kulik et al. 2009; Shi et al. 2011) and as in van Hemert \& van Dishoeck (2008). These calculated potentialenergy curves are in good agreement with the ground state and first excited ${ }^{2} \Pi$ and ${ }^{2} \Sigma^{+}$states of previous work (Lavendy et al. 1987; Kulik et al. 2009), but find the highly-excited photodissociating states to be more bound than in the previous calculations, by as much as $1 \mathrm{eV}$. This lowering arises from a larger basis set provided to the ab initio potential-energy curve calculation.

A photodissociation cross section was generated from the calculated curves and transition moments by reflecting the ground state vibrational wavefunction through each excited-state potential and onto the energy axis (Condon 1928). Scaling this reflection with the $R$-dependent transition moments and converting the potential-energy scale to wavelength generates the cross section in Fig. 18. The integrated calculated cross section is one quarter that determined by Lavendy et al. (1987) but in approximate agreement with the reanalysis of el-Qadi \& Stancil (2013), however, the increased binding of the $5^{2} \Pi$ state in our calculation leads to a $10 \mathrm{~nm}$ longwards shift of its peak cross section, to $110 \mathrm{~nm}$. The photodissociation rate assuming ISRF radiation is half of the value calculated assuming the cross section of Lavendy et al. (1987; see Sect. 5). The new rate would a factor of two smaller still without the $10 \mathrm{~nm}$ shift described.

There are no strong ultraviolet-absorbing excited states of $\mathrm{CN}$ that dissociate to form $\mathrm{C}\left({ }^{3} \mathrm{P}\right)+\mathrm{N}\left({ }^{4} \mathrm{~S}\right)$ ground state atoms, or the excited pair $\mathrm{C}\left({ }^{1} \mathrm{D}\right)+\mathrm{N}\left({ }^{4} \mathrm{~S}\right)$ (with $140 \mathrm{~nm}$ dissociation threshold). We then assumed all bound ${ }^{2} \Pi$ and ${ }^{2} \Sigma^{+}$levels dissociate with efficiency $\eta^{d}=1$ for energies above the $C\left({ }^{3} \mathrm{P}\right)+\mathrm{N}\left({ }^{2} \mathrm{D}\right)$ dissociation limit (with a $120 \mathrm{~nm}$ threshold) and all lower-energy levels have $\eta^{\mathrm{d}}=0$. It is also possible that the lower-energy bound levels are actually predissociated through spin-orbit interaction with quartet states dissociating to the $\mathrm{C}\left({ }^{3} \mathrm{P}\right)+\mathrm{N}\left({ }^{4} \mathrm{~S}\right)$ limit, with a 
Table 14. References and wavelength ranges of concatenated HCN cross sections.

\begin{tabular}{cl}
\hline \hline Wavelength $(\mathrm{nm})$ & Reference \\
\hline $63-105$ & Nuth \& Glicker $(1982)^{a}$ \\
$105-153$ & Lee (1980) \\
$153-188$ & West \& Berry (1974) \\
\hline
\end{tabular}

Notes. ${ }^{(a)}$ Scaled by a factor of $2.89 \times 10^{-20}$ to best agree with the integrated cross section of Lee (1980) where this overlaps.

Table 15. References and wavelength ranges of concatenated $\mathrm{HC}_{3} \mathrm{~N}$ cross sections.

\begin{tabular}{cl}
\hline \hline Wavelength (nm) & Reference \\
\hline $80-184$ & Ferradaz et al. (2009) \\
$184-230$ & Bénilan et al. (1994) \\
$230-255$ & Seki et al. (1996) \\
\hline
\end{tabular}

corresponding $160 \mathrm{~nm}$ threshold. Even a moderate dissociation efficiency and low cross section at such long wavelengths could increase the dissociation rate of $\mathrm{CN}$ in a cool radiation field by an order of magnitude.

\subsubsection{HCN - hydrogen cyanide}

HCN absorbs strongly at wavelengths shorter than $150 \mathrm{~nm}$ with a $100 \%$ yield of $\mathrm{H}$ and $\mathrm{CN}$ photodissociation products and two experimental cross sections for this process Lee (1980), Nuth \& Glicker (1982) were combined, as listed in Table 14. There are weaker absorption bands longwards of $150 \mathrm{~nm}$ that appear in high-resolution spectra with broadened lineshapes suggestive of predissociation-dominated lifetimes (Herzberg \& Innes 1957; Hsu et al. 1984; Jonas et al. 1990). The absorption cross section of West \& Berry (1974) was adopted for wavelengths greater than $157 \mathrm{~nm}$, and shows good agreement with the cross section of Lee (1980) where they overlap. Absorption at these long wavelengths contributes $50 \%$ of the total HCN photodissociation rate in a $4000 \mathrm{~K}$ black-body radiation field (see Sect. 5) but is not significant for hotter ultraviolet radiation fields.

$\mathrm{HCN}$ begins to photoionise to $\mathrm{HCN}^{+}$shortward of $92 \mathrm{~nm}$ and is assumed to have a $100 \%$ ionisation yield by $83 \mathrm{~nm}$. The intervening branching to dissociation and ionisation was calculated by comparing the wavelength dependence of the absorption cross section and a measured photoionisation yield (Dibeler \& Liston 1967).

\subsubsection{1. $\mathrm{HC}_{3} \mathrm{~N}$ - cyanoacetylene}

A combination of three $\mathrm{HC}_{3} \mathrm{~N}$ experimental photoabsorption cross sections provide good coverage from its threshold at $255 \mathrm{~nm}$ to $80 \mathrm{~nm}$, with sources listed in Table 15 . Additionally, a total photoionisation cross section is recorded by Leach et al. (2014) and here the remaining photoabsorption cross section was attributed to neutral photodissociation.

\subsubsection{2. $\mathrm{CH}_{3} \mathrm{OH}$ - methanol}

The photoabsorption cross section of methanol is well known and the sources of data used in our compilation are listed in Table 16. The photoabsorption and photoionisation cross
Table 16. References and wavelength ranges of concatenated $\mathrm{CH}_{3} \mathrm{OH}$ cross sections.

\begin{tabular}{cl}
\hline \hline Wavelength (nm) & Reference \\
\hline $60-107$ & Burton et al. (1992) \\
$107-165$ & Nee et al. (1985) \\
$165-220$ & Cheng et al. (2002) \\
\hline
\end{tabular}

sections measured by Burton et al. (1992) were used to determine the dissociation versus ionisation branching ratio shortwards of the $113 \mathrm{~nm}$ ionisation limit.

\subsubsection{3. $\mathrm{CH}_{3} \mathrm{CN}$ - acetonitrile}

The photoabsorption cross section of Eden et al. (2003) is adopted between 140 and $182 \mathrm{~nm}$ and the absorption coefficient of Nuth \& Glicker (1982) between 61 and $140 \mathrm{~nm}$. The latter was multiplied by $4.05 \times 10^{-20}$ to match the former over their $25 \mathrm{~nm}$ overlapping range. The photoionisation yield measured by Schwell et al. (2008) was used to determine partial ionisation and neutral dissociation cross sections.

\subsubsection{4. $\mathrm{CH}_{3} \mathrm{CHO}$ - acetaldehyde}

The photoabsorption cross section of $\mathrm{CH}_{3} \mathrm{CHO}$ is recorded at high-resolution between 116 and $350 \mathrm{~nm}$ by Limão Vieira et al. (2015). This measurement shows a weak long-wavelength absorption peak between 250 and $350 \mathrm{~nm}$ as well as a stronger and more structured region appearing between $184 \mathrm{~nm}$ and the first ionisation limit, $121.5 \mathrm{~nm}$.

The cross section at shorter wavelengths is not well constrained. Hurzeler et al. (1958) record the wavelength-dependent signal of mass $44\left(\mathrm{CH}_{3} \mathrm{CHO}^{+}\right)$and dehydrogenated mass 43 photoionisation fragments between 109 and $125 \mathrm{~nm}$. To place this measurement on an absolute scale the threshold $\mathrm{CH}_{3} \mathrm{CHO}^{+}$ ionisation cross section calculated by Vega et al. (2010) was assumed. We extrapolate the photoion signal of Hurzeler et al. (1958) to shorter wavelengths by assuming the same fall-off for both mass 44 and 43 fragments as calculated by Vega et al. (2010). Photoionisation data between 110 and $122 \mathrm{~nm}$ are missing from the laboratory measurement and a linear extrapolation to the peak of the ionisation signal at $110 \mathrm{~nm}$ is used to approximate this. All absorption not accounted for by the deduced photoionisation cross section is assumed to result in dissociation.

The cross section put together in this way, and plotted in Fig. 11, is uncertain around the ionisation threshold though physically plausible, and is superior to completely neglecting wavelengths shorter than those studied by Limão Vieira et al. (2015).

\subsubsection{5. $\mathrm{CH}_{3} \mathrm{NH}_{2}$ - methylamine}

Methylamine photoabsorbs and dissociates at wavelengths shorter than $250 \mathrm{~nm}$. A cross section was constructed from the photoabsorption measurements of Hubin-Franskin et al. (2002) (138 to $249 \mathrm{~nm}$ ), combined with their simultaneously-recorded electron-energy-loss-derived cross section ( 83 to $138 \mathrm{~nm}$ ) and another lower-resolution electron-energy loss measurement (Burton et al. 1994) shorter than $83 \mathrm{~nm}$. Some information on branching ratios to various products is available at a few wavelengths (Michael \& Noyes 1963; Gardner \& McNesby 1982).

A relative photoionisation yield is measured by $\mathrm{Hu}$ et al. (2002) between 121 and $135 \mathrm{~nm}$. This was absolutely calibrated 
with respect to the photoabsorption cross section by assuming a $100 \%$ ionisation yield at $121 \mathrm{~nm}$. This may well be an overestimate of the yield, in that case the photoionisation rates calculated here will be overestimated and the non-ionising rates too low. Hu et al. (2002) also measured the branching of photoionisation products into fragments.

\subsubsection{6. $\mathrm{NH}_{2} \mathrm{CHO}$ - formamide}

The photoabsorption cross section of $\mathrm{NH}_{2} \mathrm{CHO}$ is measured for wavelengths greater than $89 \mathrm{~nm}$ by Gingell et al. (1997), who combined a direct measurement with electron energy-loss spectroscopy. This cross section begins around $215 \mathrm{~nm}$ and peaks around $168 \mathrm{~nm}$ with some resonant structure.

The ionisation threshold of $\mathrm{NH}_{2} \mathrm{CHO}$ occurs just shortwards of the $121.3 \mathrm{~nm}$ Lyman- $\alpha$ transition. The photoion spectra of Leach et al. (2010) was converted into dissociation and ionisation cross sections by assuming $100 \%$ ionisation shorter than $89 \mathrm{~nm}$ and scaling to match the photoabsorption cross section of (Gingell et al. 1997) at this wavelength.

\subsubsection{7. $\mathrm{C}_{2} \mathrm{H}_{5} \mathrm{OH}$ - ethanol}

$\mathrm{A} \mathrm{C}_{2} \mathrm{H}_{5} \mathrm{OH}$ photoabsorption cross section measured by electronenergy loss spectroscopy (Feng \& Brion 2002) agrees well with several less-complete direct photoabsorption measurements available from the MPI Mainz UV/VIS database, and is adopted here. All absorption events are assumed to lead to dissociation.

An absolute ionisation cross section determined by photoion spectroscopy (Cool et al. 2005) was adopted between $105 \mathrm{~nm}$ and the photoionisation threshold at $120 \mathrm{~nm}$. For shorter wavelengths, 55 to $90.5 \mathrm{~nm}$, a cross section was calculated from the measured photoionisation efficiency of Hatano (1999). The photoionisation cross section was linearly interpolated between these two wavelength ranges.

\subsubsection{8. $\mathrm{C}_{3} \mathrm{H}_{7} \mathrm{OH}$ - 1-propanol}

Two measurements spanning the wavelength ranges 120 to $208 \mathrm{~nm}$ (Salahub \& Sandorfy 1971) and 30 to $120 \mathrm{~nm}$ (Koizumi et al. 1986) were combined into a single photoabsorption cross section. An absolute ionisation cross section between 125 and $107 \mathrm{~nm}$ is measured by ion mass-spectroscopy (Cool et al. 2005), and a photoionisation cross section constructed by supplementing this with a measured photoionisation efficiency between 76 and $92 \mathrm{~nm}$ (Hatano 1999). The ionisation cross section for intervening wavelengths was linearly interpolated, and linearly extrapolated to shorter wavelengths assuming a photoionisation fraction of 1 shortwards of $60 \mathrm{~nm}$, in line with similar molecules (Hatano 1999).

\subsubsection{OCS - carbonyl sulphide}

The photoabsorption of OCS is studied extensively in view of its importance to atmospheric chemistry (e.g., Limão Vieira et al. 2015, and references therein). Here, the recent high-resolution measurement of its cross section by Limão Vieira et al. (2015) was adopted, and supplemented with photoabsorption and ionisation cross sections deduced form electron-energy loss spectroscopy for wavelengths shorter than $115 \mathrm{~nm}$ (Feng et al. 2000b,a).
Table 17. References and wavelength ranges of concatenated $\mathrm{CS}_{2}$ cross sections.

\begin{tabular}{cl}
\hline \hline Wavelength $(\mathrm{nm})$ & Reference \\
\hline $18-78$ & Wu \& Judge (1983) \\
$78-97$ & Cook \& Ogawa (1969) \\
$97-105$ & Carnovale et al. (1981) \\
$105-121$ & Day et al. (1982) \\
$121-193$ & Sunanda et al. (2015) \\
$193-205$ & Xu \& Joens (1993) \\
$205-370$ & Grosch et al. (2015) \\
\hline
\end{tabular}

Notes. ${ }^{(a)}$ Scaled by a factor of 1.27 to agree with the integrated cross section of Wu \& Judge (1983) for their overlapping wavelength region. (b) Scaled by a factor of 0.87 to agree with the integrated cross section of Wu \& Judge (1983) for their overlapping wavelength region.

\subsubsection{0. $\mathrm{CH}_{3} \mathrm{SH}$ - methanethiol}

Two experimental cross sections (Vaghjiani 1993; Tokue et al. 1987) were combined to describe $\mathrm{CH}_{3} \mathrm{SH}$ photoabsorption from its long-wavelength threshold at $330 \mathrm{~nm}$ (Wilson et al. 1994) to the ionisation threshold at $131 \mathrm{~nm}$ (Morgan et al. 1995). For shorter wavelengths, a photodissociation cross section linearly extrapolated to zero at $100 \mathrm{~nm}(30 \mathrm{~nm}$ below the ionisation threshold) was adopted, and a photoionisation cross section using the measured efficiency for $\mathrm{CH}_{3} \mathrm{SH}^{+}$production of Kutina et al. (1982) after scaling its maximum value to $5 \times 10^{-18} \mathrm{~cm}^{2}$. The various assumptions used in the shorter wavelength region were selected in a broad analogy to the photodissociation and ionisation properties of $\mathrm{CH}_{3} \mathrm{OH}$.

\subsubsection{CS - carbon monosulphide}

There are no absolute measurements of the CS cross section for photoabsorption into pre- or directly-dissociative excited states. Here, the cross section simulated and discussed by van Dishoeck (1988) was retained. This uses the measured wavelengths of transitions to the $B^{1} \Sigma^{+}$states (Stark et al. 1987) and vertical excitation energies of higher-lying states calculated by Bruna et al. (1975). The strengths of these electronic transitions are estimated.

\subsubsection{2. $\mathrm{CS}_{2}$ - carbon disulphide}

Multiple experiments were combined into a single $\mathrm{CS}_{2}$ photoabsorption cross section spanning 20 to $370 \mathrm{~nm}$, with details given in Table 17. The ionisation and ground-state dissociation thresholds are at 123 and $277 \mathrm{~nm}$, respectively (Fischer et al. 1993; Okabe 1972). A photoionisation cross section was deduced by scaling the photoionisation efficiency measured by Dibeler \& Walker (1967) to match the photoabsorption cross section of Wu \& Judge (1983) for wavelengths sufficiently shorter than the ionisation threshold that non-ionising decay is negligible.

\subsubsection{3. $\mathrm{SO}_{2}-$ sulphur dioxide}

The photoabsorption cross section of $\mathrm{SO}_{2}$ was measured numerous times and a consolidation of these into a single spectrum between 106 and $403 \mathrm{~nm}$ was generated by Manatt \& Lane (1993). This was modified by inserting a higher-resolution measured cross section between 172 and $289 \mathrm{~nm}$, including the important 
photodissociating absorption bands between $170 \mathrm{~nm}$ and the photodissociation threshold at $218.7 \mathrm{~nm}$ (Becker et al. 1995). A measured fluorescence yield (Katagiri et al. 1997) was used to estimate the dissociation fraction near this threshold. Evenhigher resolution measurements of these bands are available (e.g., Blackie et al. 2011; Endo et al. 2015) but were deemed unnecessary for our purposes.

A combination of photoionisation efficiency and shortwavelength photoabsorption cross sections measured by electron-impact and photoion spectroscopy (Feng et al. 1999c; Holland et al. 1995) were used to determine the absorption and ionisation cross sections for wavelengths shorter than compiled by Manatt \& Lane (1993).

\subsubsection{4. $\mathrm{SH}^{+}-$mercapto ion}

The direct photodissociation cross section of $\mathrm{SH}^{+}$is calculated ab initio by McMillan et al. (2016) including transitions to several excited states. Their calculation from the $v=0$ and $J=0$ ground-state level was adopted here and added to this additional absorption into the longer-wavelength bound-levels of the $A^{3} \Pi$ state, which is known to predissociate for $v \geq 1$ (Gustafsson et al. 1988; Brites et al. 2008). Oscillator strength of transitions into the vibrational levels of $A^{3} \Pi$ were calculated here using the potential-energy curves and transition dipole moments of McMillan et al. (2016) and the methods of Sect. 4.3.49, but did not include details of their rotational structure.

\subsubsection{SO - sulphur monoxide}

The predissociated $B^{3} \Sigma^{-}-X^{3} \Sigma^{-}$absorption bands of SO, appearing shortward of $235 \mathrm{~nm}$, are measured by Phillips (1981). The short wavelength absorption between 116 and $135 \mathrm{~nm}$ measured by Nee \& Lee (1986) was added to this and a relative photoionisation yield shortwards of $121 \mathrm{~nm}$ (Norwood \& Ng 1989) that we arbitrarily scaled to give a peak value of $5 \times 10^{-17} \mathrm{~cm}^{2}$.

\subsubsection{6. $\mathrm{S}_{2}-$ disulphur}

The photodissociation and ionisation cross section of the $\mathrm{S}_{2}$ radical is not well known, but is important for understanding $\mathrm{S}$-bearing molecular abundances evident in comets (de Almeida \& Singh 1986). Photodissociating transitions occur into the $B^{3} \Sigma_{u}^{-}$state, which is predissociated for levels $v \geq 10$ (Kato \& Baba 1995). Transition wavelengths for $B\left(v^{\prime}\right) \leftarrow X(0)$ photoabsorption bands were calculated using the molecular constants of Wheeler et al. (1998) and adopted oscillator strengths for $v^{\prime}=0$ to 20 bands from the calculations of Pradhan et al. (1991). These oscillator strengths to $v^{\prime}=26$ were extrapolated using Franck-Condon factors calculated for the $B \leftarrow X$ transition by Smith \& Liszt (1971). According to these factors, vibrational levels with $v^{\prime}>26$ will contribute less than $6 \%$ to the total absorption into the $B$ state, and the continuum absorption shorter than the $224 \mathrm{~nm}$ direct dissociation limit will be weak. The photoabsorption and photodissociation cross sections simulated here neglect rotational structure of the individual vibrational bands (e.g., Wheeler et al. 1998).

The photoionisation efficiency of $\mathrm{S}_{2}$ is measured (Liao \& $\mathrm{Ng}$ 1986) and a typical absolute magnitude for this was arbitrarily assumed here. Additional bound and unbound states exist between the $B^{3} \Sigma_{u}^{-}$and ionisation continuum (e.g., Donovan et al. 1970; Xing et al. 2013) but no quantitative information on their photoabsorption cross sections is available. It is likely that the longer-wavelength absorption of $B^{3} \Sigma_{u}^{-}$will dominate the photodissociation rate for $\mathrm{S}_{2}$ in most interstellar radiation fields.

Foreshadowing Sect. 5, we calculate the photodissociation rate of $S_{2}$ in the solar radiation field at $1 \mathrm{AU}$ distance from the Sun to be $0.0079 \mathrm{~s}^{-1}$, that is with a lifetime of $130 \mathrm{~s}$, which is a significant reduction from the $250 \mathrm{~s}$ lifetime calculated by de Almeida \& Singh (1986). This difference is likely due to the larger number of predissociating $B^{3} \Sigma_{u}^{-}$vibrational levels considered in the present work, leading to a larger total photodissociation rate.

\subsubsection{7. $\mathrm{SH}$ - mercapto radical}

Several ab initio calculations of the SH ground and excited states have been made (Bruna \& Hirsch 1987; Resende \& Ornellas 2001; Lee et al. 2001), and there is one absolute absorption measurement, providing the oscillator strength of the predissociative $X^{2} \Pi\left(v^{\prime \prime}=0\right)$ to $A^{2} \Sigma^{+}\left(v^{\prime}=0\right)$ transition. This measurement was combined with calculated Franck-Condon factors for transition to higher- $v^{\prime \prime} A^{2} \Sigma^{+}$levels (Resende \& Ornellas 2001) and experimental spectroscopic constants (Johns \& Ramsay 1961) to estimate the cross section of $A-X$ transitions (neglecting their rotational structure). Furthermore, a "reflection" cross section to simulate continuum absorption into the lowest-energy repulsive ${ }^{2} \Sigma^{-}$state was added, using the potential-energy curves and electronic transition moment of Lee et al. (2001), and the methods described in Sect. 4.3.49. Higher lying bound and repulsive states were included as vertical transitions according to their calculated equilibrium energies and transition moments (Bruna \& Hirsch 1987). Finally, the unknown photoionisation cross section of $\mathrm{SH}$ was arbitrarily simulated by including an absorption feature of integrated cross section $5 \times 10^{-17} \mathrm{~cm}^{2}$ shortwards of the $119 \mathrm{~nm}$ photoionisation threshold (Hsu et al. 1994).

\subsubsection{8. $\mathrm{H}_{2} \mathrm{~S}$ - hydrogen sulphide}

There are several measurements of the photoabsorption of $\mathrm{H}_{2} \mathrm{~S}$, and data from Lee et al. (1987) (240 to $118.8 \mathrm{~nm}$ ), Xia et al. (1991) (118.8 to $106 \mathrm{~nm}$ ), and Feng et al. (1999a) (106 to $41 \mathrm{~nm}$ ), was compiled here into a single cross section. It is likely that some of the absorption features between 120 and $160 \mathrm{~nm}$ are not fully resolved by these measurements. It was assumed that all absorption leads to dissociation or ionisation, with the branching between these two determined from the photoionisation cross sections of Xia et al. (1991), Feng et al. (1999b).

\subsubsection{9. $\mathrm{SiO}$ - silicon monoxide}

This molecule has a similar electronic structure to that of $\mathrm{CO}$, but with a lower dissociation energy, $8.26 \mathrm{eV}$ vs. $11 \mathrm{eV}$. Thus, even the lower ${ }^{1} \Sigma^{+}$Rydberg states can contribute to $\mathrm{SiO}$ photodissociation whereas they are bound for $\mathrm{CO}$ (e.g., its B and $\mathrm{C} v=0$ levels). Another difference between the two molecules are quite distinct ionisation potentials: $11.4 \mathrm{eV}$ for $\mathrm{SiO}$ and $14 \mathrm{eV}$ for $\mathrm{CO}$, putting the latter above the Lyman-limit. The oscillator strengths to the $3^{1} \Sigma^{+}$and 2, 3, 4 and $5^{1} \Pi$ states are taken to be $f=0.10$, $0.32,0.03,0.11$ and 0.10 , respectively, with $\eta^{\mathrm{d}}=1$ as deduced by ab initio calculations (van Dishoeck et al. 2006).

\subsubsection{0. $\mathrm{HCl}$ - hydrogen chloride}

The $\mathrm{HCl}$ photoabsorption cross section was adopted from the combination of a direct measurement (Bahou et al. 2001) 
and electron-energy loss spectroscopy (Brion et al. 2005) over the 5 to 135 , and 135 to $230 \mathrm{~nm}$ wavelength regions, respectively. A photoionisation cross section is recorded by Frohlich \& Glass-Maujean (1990). The $\mathrm{HCl}$ spectrum spans a region of continuous absorption into the repulsive $A^{1} \Pi$ state between 135 and $250 \mathrm{~nm}$, many bound excited Rydberg and valence levels between 100 and $135 \mathrm{~nm}$, and an ionisation continuum shortwards of $97.2 \mathrm{~nm}$.

The most recent ab initio calculation of the $\mathrm{HCl}$ cross section by Engin et al. (2012) identifies 13 photoabsorbing electronically excited states of ${ }^{1} \Pi$ and ${ }^{1} \Sigma^{+}$symmetry below $12.2 \mathrm{eV}$, and is excellent in agreement with the previous computation of van Dishoeck et al. (1982) for those states absorbing longer than $120 \mathrm{~nm}$. Comparing the integrated cross section of Engin et al. (2012) and the experimental data between 102 and $200 \mathrm{~nm}$ leads to agreement within $4 \%$. The excited states responsible for the significant $\mathrm{HCl}$ absorption between 91.6 and $102 \mathrm{~nm}$ are not well known. Many bands are observed and assigned to Rydberg-type excited states in this region (Ginter \& Ginter 1981; Green et al. 1991) but without quantification of their absorption cross sections. An independent measurement of the $\mathrm{HCl}$ photoabsorption spectrum below $110 \mathrm{~nm}$ is warranted.

All photoabsorbing states were here assumed to be completely dissociative $\left(\eta^{\mathrm{d}}=1\right)$, despite their sometimes unbroadened linewidths. This is based on the weakness of the fluorescence cross section from these states (Nee et al. 1986) and the strong coupling of Rydberg levels and unbound valence states in theoretical studies (e.g., van Dishoeck et al. 1982; Alexander et al. 1998).

Shorter than the $96 \mathrm{~nm}$ ionisation limit, a small signal of $\mathrm{H}^{+}$or $\mathrm{Cl}^{+}$dissociative-photoionisation products is measured (Daviel et al. 1984), as well as a small cross section for decay into neutral products (Frohlich \& Glass-Maujean 1990).

\subsubsection{1. $\mathrm{HCl}^{+}$- hydrogen chloride ion}

$\mathrm{Ab}$ initio calculation of potential-energy curves for the $\mathrm{HCl}^{+}$ground and lowest-excited states, and the transition moments connecting them, permitted a calculation of the molecule's wavelength-dependent photodissociation cross section (Pradhan et al. 1991). The dominant dissociation pathway determined by this calculation produces $\mathrm{H}$ and $\mathrm{Cl}^{+}$.

\subsubsection{AlH - alumane}

Potential-energy curves for the $A^{1} \Sigma^{+}$and $C^{1} \Sigma^{+}$states of $\mathrm{AlH}$ are calculated by Matos et al. (1987) as well as electric-dipole transition moments with respect to the $X^{1} \Sigma^{+}$ground state. Vertical transitions calculated from this data at the equilibrium geometry give absorption at 430 and $230 \mathrm{~nm}$ with oscillator strengths of 0.0022 and 0.090 , respectively. This however represents an upper limit for photodissociation because unbroadened emission lines from the $v=0$ and 1 levels of both the $A$ and $C$ states is observed (Szajna \& Zachwieja 2010; Szajna et al. 2011). The predissociation of higher energy vibrational structure for both states may be possible however if they are able to tunnel through maxima predicted for both potential-energy curves (Matos et al. 1987; Bauschlicher \& Langhoff 1988). Given this uncertainty, two absorption features were assumed here to represent the $\mathrm{AlH}$ spectrum with oscillator strengths of 0.05 , one at $200 \mathrm{~nm}$ to represent all photodissociating states, and another at $130 \mathrm{~nm}$ to for photoionisation, the photoionisation threshold occurs at approximately $160 \mathrm{~nm}$ (Matos et al. 1987). The ISRF rate is a factor of
10 smaller than previously given in the Leiden database because of the lack of dissociation via the $v=0$ and 1 levels.

\section{Photodestruction due to stellar and interstellar radiation}

\subsection{Results}

Photodestruction rates were calculated according to Eq. (1) using the cross sections discussed in Sect. 4 and assuming several of the radiation fields detailed in Sect. 2, selected to represent a range of astrochemical environments. Tables 18 and 19 summarise the calculated rates for photodissociation and photoionisation, respectively. The photodissociation and photoionisation rates in the ISRF have uncertainties listed in Table 1 and described in Sect. 4.1. These can be incorporated into chemical rate networks to perform sensitivity analyses of model abundances (Wakelam et al. 2012). For cool radiation fields, such as a $4000 \mathrm{~K}$ black body or the solar radiation field, low-lying molecular states just above the dissociation limit dominate the photodissociation rate and uncertainty. For example, the interstellar rate of $\mathrm{OH}$ is dictated by three direct and continuous channels in the 190 to $91.2 \mathrm{~nm}$ range, whereas the rate assuming $4000 \mathrm{~K}$ blackbody radiation proceeds primarily by predissociation through the $A^{2} \Sigma^{+}$state around $300 \mathrm{~nm}$ (van Dishoeck \& Dalgarno 1984b; van Dishoeck et al. 1984).

We also computed photodestruction rates assuming the radiation field of Mathis et al. (1983) (10 kpc Galactocentric distance). These are consistently 30 to $40 \%$ smaller than for our standard ISRF due to the differing magnitudes but similar shape of the two radiation fields over the range 100 to $300 \mathrm{~nm}$. For the species (about 15\%) that are primarily photodestroyed at wavelengths between the Lyman-limit and $100 \mathrm{~nm}$ (e.g., dissociation for many diatomics or ionisation of many species) the Mathis 1983 rates are more similar or occasionally greater.

The photodissociation and photoionisation rates for all atoms and molecules in our database are compared in Figs. 19 and 20 assuming exposure to three different wavelength-dependent radiation fields. These are plotted in order of decreasing ISRF rate and with varying vertical scales. The largest ISRF photodissociation rate is $8.5 \times 10^{-9} \mathrm{~s}^{-1}$ and occurs for $\ell-\mathrm{C}_{4}$, while the smallest rate is $5.4 \times 10^{-12} \mathrm{~s}^{-1}$ for $\mathrm{HCO}^{+}$. These extremes correspond to lifetimes against photodissociation of 4 to 6000 years, respectively. The lowest rates occur mostly for small ions, while neutral molecules mostly fall between 0.5 and $4 \times 10^{-9} \mathrm{~s}^{-1}$.

The photoionisation rates of most molecules exposed to the ISRF are between 2 and 10 times smaller than their respective photodissociation rates, but can be many times smaller still. This provides some reassurance that the dominant photodestruction pathway is included in our database for those molecules where we evaluated a photodissociation rate but neglected the corresponding photoionisation cross section and rate because of a lack of reliable information. For two molecules, $\mathrm{C}_{2}$ and $\mathrm{CH}_{3} \mathrm{NH}_{2}$, that have particularly large cross section and long wavelength thresholds for photoionisation the ISRF photoionisation rate is actually larger than for photodissociation, by factors of 1.7 and 2.2 , respectively.

The response of the various molecules to alternative radiation fields is highly variable and largely controlled by the qualitative properties of their wavelength-dependent cross sections. Comparative rates are plotted in Figs. 19 and 20. We note that these are calculated assuming radiation fields with equal integrated energy intensity between 91.2 and $200 \mathrm{~nm}$, while the intensity in real astrochemical environments of interest varies by 
Table 18. Photodissociation rates of molecules and parameterised dust shielding.

\begin{tabular}{|c|c|c|c|c|c|c|c|c|c|c|}
\hline \multirow[b]{2}{*}{ Species } & \multirow[b]{2}{*}{ ISRF } & \multirow[b]{2}{*}{ Mathis '83 } & \multirow[b]{2}{*}{$4000 \mathrm{~K}$} & \multirow[b]{2}{*}{$10000 \mathrm{~K}$} & \multirow[b]{2}{*}{ Lyman- $\alpha$} & \multirow[b]{2}{*}{ Solar } & \multirow[b]{2}{*}{ TW-Hydra } & \multicolumn{3}{|c|}{ ISRF dust shielding } \\
\hline & & & & & & & & $\gamma_{\text {exp,ISM }}$ & $\gamma_{\mathrm{E}_{2}, \mathrm{ISM}}$ & $\gamma_{\mathrm{E}_{2}, \text { growth }}$ \\
\hline $\mathrm{H}_{2}$ & $5.7 \mathrm{E}-11$ & $4.6 \mathrm{E}-11$ & $2.0 \mathrm{E}-16$ & $3.9 \mathrm{E}-12$ & - & $3.1 \mathrm{E}-12$ & $1.7 \mathrm{E}-11$ & 4.18 & 3.11 & 0.45 \\
\hline $\mathrm{H}_{2}^{+}$ & $5.7 \mathrm{E}-10$ & $3.9 \mathrm{E}-10$ & $4.3 \mathrm{E}-11$ & $2.0 \mathrm{E}-10$ & $1.1 \mathrm{E}-09$ & $8.7 \mathrm{E}-11$ & $7.6 \mathrm{E}-10$ & 2.78 & 1.94 & 0.44 \\
\hline $\mathrm{H}_{3}^{+}$ & - & - & - & - & - & $3.9 \mathrm{E}-13$ & $3.7 \mathrm{E}-12$ & - & - & - \\
\hline $\mathrm{CH}$ & $9.1 \mathrm{E}-10$ & $6.4 \mathrm{E}-10$ & $1.8 \mathrm{E}-07$ & $2.2 \mathrm{E}-09$ & $7.9 \mathrm{E}-12$ & $2.1 \mathrm{E}-07$ & $1.1 \mathrm{E}-09$ & 2.12 & 1.36 & 0.32 \\
\hline $\mathrm{CH}^{+}$ & $3.3 \mathrm{E}-10$ & $2.6 \mathrm{E}-10$ & $6.6 \mathrm{E}-10$ & $3.8 \mathrm{E}-11$ & $7.9 \mathrm{E}-12$ & $9.0 \mathrm{E}-10$ & $8.1 \mathrm{E}-11$ & 3.54 & 2.63 & 0.45 \\
\hline $\mathrm{CH}_{2}$ & $5.8 \mathrm{E}-10$ & $3.9 \mathrm{E}-10$ & $3.0 \mathrm{E}-09$ & $1.3 \mathrm{E}-09$ & $7.9 \mathrm{E}-12$ & $3.5 \mathrm{E}-09$ & $3.7 \mathrm{E}-10$ & 2.35 & 1.61 & 0.31 \\
\hline $\mathrm{CH}_{2}^{+}$ & $1.4 \mathrm{E}-10$ & $1.2 \mathrm{E}-10$ & $3.7 \mathrm{E}-11$ & $8.3 \mathrm{E}-11$ & $7.9 \mathrm{E}-12$ & $3.4 \mathrm{E}-11$ & $4.7 \mathrm{E}-11$ & 2.73 & 1.86 & 0.40 \\
\hline $\mathrm{CH}_{3}$ & $6.2 \mathrm{E}-10$ & $4.0 \mathrm{E}-10$ & $2.6 \mathrm{E}-09$ & $7.5 \mathrm{E}-10$ & - & $4.3 \mathrm{E}-09$ & $2.3 \mathrm{E}-10$ & 2.50 & 1.73 & 0.38 \\
\hline $\mathrm{CH}_{4}$ & $1.5 \mathrm{E}-09$ & $1.1 \mathrm{E}-09$ & $1.7 \mathrm{E}-12$ & $2.7 \mathrm{E}-10$ & $2.8 \mathrm{E}-09$ & $1.3 \mathrm{E}-10$ & $2.0 \mathrm{E}-09$ & 3.08 & 2.19 & 0.45 \\
\hline $\mathrm{CH}_{4}^{+}$ & $2.8 \mathrm{E}-10$ & $1.9 \mathrm{E}-10$ & $1.7 \mathrm{E}-13$ & $4.6 \mathrm{E}-11$ & $7.9 \mathrm{E}-12$ & $3.7 \mathrm{E}-12$ & $5.2 \mathrm{E}-11$ & 3.11 & 2.22 & 0.45 \\
\hline $\mathrm{C}_{2}^{4}$ & $2.4 \mathrm{E}-10$ & $1.7 \mathrm{E}-10$ & $4.6 \mathrm{E}-13$ & $4.5 \mathrm{E}-11$ & $7.8 \mathrm{E}-11$ & 7.3E-12 & $9.2 \mathrm{E}-11$ & 3.04 & 2.15 & 0.45 \\
\hline $\mathrm{C}_{2} \mathrm{H}$ & $1.6 \mathrm{E}-09$ & $1.0 \mathrm{E}-09$ & $4.4 \mathrm{E}-11$ & $6.8 \mathrm{E}-10$ & $5.1 \mathrm{E}-10$ & $6.5 \mathrm{E}-11$ & $8.6 \mathrm{E}-10$ & 2.67 & 1.85 & 0.45 \\
\hline $\mathrm{C}_{2} \mathrm{H}_{2}$ & $2.4 \mathrm{E}-09$ & $1.6 \mathrm{E}-09$ & $1.3 \mathrm{E}-10$ & $1.1 \mathrm{E}-09$ & $8.6 \mathrm{E}-09$ & $4.2 \mathrm{E}-10$ & $3.2 \mathrm{E}-09$ & 2.64 & 1.83 & 0.45 \\
\hline $\mathrm{C}_{2} \mathrm{H}_{4}$ & $3.1 \mathrm{E}-09$ & $2.0 \mathrm{E}-09$ & $7.5 \mathrm{E}-10$ & $2.4 \mathrm{E}-09$ & $3.7 \mathrm{E}-09$ & $7.9 \mathrm{E}-10$ & $3.5 \mathrm{E}-09$ & 2.49 & 1.70 & 0.40 \\
\hline $\mathrm{C}_{2} \mathrm{H}_{6}^{4}$ & $2.1 \mathrm{E}-09$ & $1.5 \mathrm{E}-09$ & $5.9 \mathrm{E}-12$ & $4.8 \mathrm{E}-10$ & $3.6 \mathrm{E}-09$ & $1.6 \mathrm{E}-10$ & $2.5 \mathrm{E}-09$ & 2.94 & 2.07 & 0.45 \\
\hline $\mathrm{C}_{3}$ & $5.0 \mathrm{E}-09$ & $3.2 \mathrm{E}-09$ & $6.5 \mathrm{E}-10$ & $4.3 \mathrm{E}-09$ & $2.2 \mathrm{E}-09$ & $5.8 \mathrm{E}-10$ & $4.2 \mathrm{E}-09$ & 2.39 & 1.64 & 0.43 \\
\hline$l-\mathrm{C}_{3}^{3} \mathrm{H}$ & $1.8 \mathrm{E}-09$ & $1.2 \mathrm{E}-09$ & $4.7 \mathrm{E}-07$ & $4.4 \mathrm{E}-09$ & $7.9 \mathrm{E}-12$ & $4.2 \mathrm{E}-07$ & $2.1 \mathrm{E}-09$ & 2.08 & 1.32 & 0.31 \\
\hline$c-\mathrm{C}_{3} \mathrm{H}$ & $1.1 \mathrm{E}-09$ & $6.5 \mathrm{E}-10$ & $1.1 \mathrm{E}-07$ & $3.8 \mathrm{E}-09$ & $7.9 \mathrm{E}-12$ & $1.3 \mathrm{E}-07$ & $1.1 \mathrm{E}-09$ & 2.15 & 1.45 & 0.27 \\
\hline $\mathrm{HC}_{3}^{3} \mathrm{H}$ & $2.2 \mathrm{E}-09$ & $1.5 \mathrm{E}-09$ & $1.8 \mathrm{E}-07$ & $6.5 \mathrm{E}-09$ & 7.9E-12 & $2.9 \mathrm{E}-07$ & $2.2 \mathrm{E}-09$ & 2.15 & 1.43 & 0.29 \\
\hline$l-\mathrm{C}_{3} \mathrm{H}_{2}$ & $4.1 \mathrm{E}-09$ & $2.7 \mathrm{E}-09$ & $6.7 \mathrm{E}-09$ & $3.7 \mathrm{E}-09$ & $2.3 \mathrm{E}-09$ & 7.2E-09 & $3.2 \mathrm{E}-09$ & 2.51 & 1.74 & 0.40 \\
\hline$c-\mathrm{C} 3 \mathrm{H} 2$ & $1.4 \mathrm{E}-09$ & $8.8 \mathrm{E}-10$ & $5.5 \mathrm{E}-08$ & $3.3 \mathrm{E}-09$ & $7.9 \mathrm{E}-12$ & $3.0 \mathrm{E}-08$ & $8.5 \mathrm{E}-10$ & 2.26 & 1.54 & 0.31 \\
\hline$l-\mathrm{C}_{4}$ & $8.5 \mathrm{E}-09$ & $5.7 \mathrm{E}-09$ & $5.4 \mathrm{E}-08$ & $2.2 \mathrm{E}-08$ & $7.9 \mathrm{E}-12$ & $8.4 \mathrm{E}-08$ & $5.5 \mathrm{E}-09$ & 2.22 & 1.52 & 0.29 \\
\hline$l-\mathrm{C}_{4} \mathrm{H}$ & $3.7 \mathrm{E}-09$ & $2.3 \mathrm{E}-09$ & $7.4 \mathrm{E}-10$ & $3.3 \mathrm{E}-09$ & $7.9 \mathrm{E}-12$ & $6.8 \mathrm{E}-10$ & $1.2 \mathrm{E}-09$ & 2.36 & 1.62 & 0.43 \\
\hline$l-\mathrm{C}_{5}^{4} \mathrm{H}$ & $1.3 \mathrm{E}-09$ & $9.4 \mathrm{E}-10$ & $6.9 \mathrm{E}-07$ & $8.0 \mathrm{E}-09$ & $7.9 \mathrm{E}-12$ & $9.6 \mathrm{E}-07$ & $2.3 \mathrm{E}-09$ & 1.76 & 1.14 & 0.22 \\
\hline $\mathrm{OH}$ & $3.8 \mathrm{E}-10$ & $2.5 \mathrm{E}-10$ & $1.9 \mathrm{E}-10$ & $2.0 \mathrm{E}-10$ & $6.4 \mathrm{E}-10$ & $1.7 \mathrm{E}-10$ & $5.1 \mathrm{E}-10$ & 2.66 & 1.83 & 0.43 \\
\hline $\mathrm{OH}^{+}$ & $1.3 \mathrm{E}-11$ & $1.1 \mathrm{E}-11$ & $4.4 \mathrm{E}-13$ & $9.6 \mathrm{E}-13$ & - & $6.8 \mathrm{E}-13$ & $2.9 \mathrm{E}-12$ & 3.97 & 2.96 & 0.45 \\
\hline $\mathrm{H}_{2} \mathrm{O}$ & $7.7 \mathrm{E}-10$ & $5.3 \mathrm{E}-10$ & $1.6 \mathrm{E}-10$ & $4.6 \mathrm{E}-10$ & $2.4 \mathrm{E}-09$ & $2.3 \mathrm{E}-10$ & $1.6 \mathrm{E}-09$ & 2.63 & 1.80 & 0.41 \\
\hline $\mathrm{O}_{2}$ & $7.7 \mathrm{E}-10$ & $5.0 \mathrm{E}-10$ & $7.5 \mathrm{E}-11$ & $5.6 \mathrm{E}-10$ & $3.2 \mathrm{E}-11$ & $6.4 \mathrm{E}-11$ & $3.7 \mathrm{E}-10$ & 2.45 & 1.69 & 0.43 \\
\hline $\mathrm{O}_{2}^{+}$ & $3.5 \mathrm{E}-11$ & $2.3 \mathrm{E}-11$ & $1.4 \mathrm{E}-11$ & $3.9 \mathrm{E}-11$ & $4.0 \mathrm{E}-10$ & $2.4 \mathrm{E}-11$ & $2.4 \mathrm{E}-10$ & 2.38 & 1.62 & 0.37 \\
\hline $\mathrm{HO}_{2}$ & $6.7 \mathrm{E}-10$ & $4.4 \mathrm{E}-10$ & $1.8 \mathrm{E}-08$ & $2.0 \mathrm{E}-09$ & 7.9E-12 & $2.2 \mathrm{E}-08$ & $4.8 \mathrm{E}-10$ & 2.46 & 1.69 & 0.28 \\
\hline $\mathrm{H}_{2} \mathrm{O}_{2}^{2}$ & $8.1 \mathrm{E}-10$ & $5.3 \mathrm{E}-10$ & $2.4 \mathrm{E}-09$ & $5.5 \mathrm{E}-10$ & $1.5 \mathrm{E}-09$ & $2.9 \mathrm{E}-09$ & $1.1 \mathrm{E}-09$ & 2.61 & 1.80 & 0.41 \\
\hline $\mathrm{O}_{3}$ & $1.8 \mathrm{E}-09$ & $1.1 \mathrm{E}-09$ & $2.4 \mathrm{E}-07$ & $5.8 \mathrm{E}-09$ & $4.7 \mathrm{E}-09$ & $2.2 \mathrm{E}-07$ & $4.1 \mathrm{E}-09$ & 2.25 & 1.49 & 0.28 \\
\hline $\mathrm{CO}$ & $2.4 \mathrm{E}-10$ & $2.1 \mathrm{E}-10$ & $2.8 \mathrm{E}-15$ & $1.8 \mathrm{E}-11$ & - & $8.0 \mathrm{E}-12$ & $5.1 \mathrm{E}-11$ & 3.88 & 2.88 & 0.45 \\
\hline $\mathrm{CO}^{+}$ & $1.0 \mathrm{E}-10$ & $7.1 \mathrm{E}-11$ & $1.7 \mathrm{E}-13$ & $2.4 \mathrm{E}-11$ & $7.9 \mathrm{E}-12$ & $1.4 \mathrm{E}-12$ & $2.3 \mathrm{E}-11$ & 2.89 & 2.04 & 0.45 \\
\hline $\mathrm{CO}_{2}$ & $9.2 \mathrm{E}-10$ & $6.8 \mathrm{E}-10$ & $2.4 \mathrm{E}-12$ & $1.1 \mathrm{E}-10$ & $1.0 \mathrm{E}-11$ & $1.7 \mathrm{E}-11$ & $1.8 \mathrm{E}-10$ & 3.40 & 2.48 & 0.45 \\
\hline $\mathrm{HCO}$ & $1.1 \mathrm{E}-09$ & $4.9 \mathrm{E}-10$ & $5.0 \mathrm{E}-06$ & $2.7 \mathrm{E}-09$ & $1.2 \mathrm{E}-11$ & $1.7 \mathrm{E}-08$ & $7.4 \mathrm{E}-09$ & 2.43 & 1.67 & 0.31 \\
\hline $\mathrm{HCO}^{+}$ & $5.4 \mathrm{E}-12$ & $3.7 \mathrm{E}-12$ & $1.1 \mathrm{E}-16$ & $4.9 \mathrm{E}-13$ & - & $2.8 \mathrm{E}-14$ & $7.2 \mathrm{E}-13$ & 3.67 & 2.68 & 0.45 \\
\hline $\mathrm{H}_{2} \mathrm{CO}$ & $1.4 \mathrm{E}-09$ & $9.6 \mathrm{E}-10$ & $4.0 \mathrm{E}-09$ & $9.6 \mathrm{E}-10$ & $1.5 \mathrm{E}-09$ & $5.1 \mathrm{E}-09$ & $1.4 \mathrm{E}-09$ & 2.54 & 1.74 & 0.42 \\
\hline $\mathrm{NH}$ & $5.7 \mathrm{E}-10$ & $3.8 \mathrm{E}-10$ & $1.1 \mathrm{E}-11$ & $2.4 \mathrm{E}-10$ & $4.9 \mathrm{E}-11$ & $1.7 \mathrm{E}-11$ & $1.7 \mathrm{E}-10$ & 2.63 & 1.83 & 0.45 \\
\hline $\mathrm{NH}^{+}$ & $5.3 \mathrm{E}-11$ & $3.6 \mathrm{E}-11$ & $1.2 \mathrm{E}-08$ & $2.0 \mathrm{E}-10$ & $7.4 \mathrm{E}-12$ & $8.4 \mathrm{E}-09$ & $5.9 \mathrm{E}-11$ & 2.07 & 1.34 & 0.26 \\
\hline $\mathrm{NH}_{2}$ & $9.5 \mathrm{E}-10$ & $6.3 \mathrm{E}-10$ & $5.5 \mathrm{E}-10$ & $1.3 \mathrm{E}-09$ & $4.8 \mathrm{E}-12$ & $4.3 \mathrm{E}-10$ & $4.4 \mathrm{E}-10$ & 2.31 & 1.57 & 0.35 \\
\hline $\mathrm{NH}_{3}$ & $1.4 \mathrm{E}-09$ & $9.9 \mathrm{E}-10$ & $3.6 \mathrm{E}-09$ & $1.7 \mathrm{E}-09$ & $1.3 \mathrm{E}-09$ & $4.1 \mathrm{E}-09$ & $1.4 \mathrm{E}-09$ & 2.61 & 1.80 & 0.36 \\
\hline $\mathrm{N}_{2}^{3}$ & $1.7 \mathrm{E}-10$ & $1.5 \mathrm{E}-10$ & $3.2 \mathrm{E}-16$ & $1.1 \mathrm{E}-11$ & - & $1.1 \mathrm{E}-11$ & $5.2 \mathrm{E}-11$ & 4.25 & 3.16 & 0.45 \\
\hline $\mathrm{NO}$ & $3.8 \mathrm{E}-10$ & $2.7 \mathrm{E}-10$ & $2.1 \mathrm{E}-10$ & $3.1 \mathrm{E}-10$ & $7.5 \mathrm{E}-11$ & $2.1 \mathrm{E}-10$ & $2.2 \mathrm{E}-10$ & 2.56 & 1.75 & 0.40 \\
\hline $\mathrm{NO}_{2}$ & $1.4 \mathrm{E}-09$ & $9.2 \mathrm{E}-10$ & $4.8 \mathrm{E}-10$ & $1.1 \mathrm{E}-09$ & 7.9E-12 & $4.1 \mathrm{E}-10$ & $1.3 \mathrm{E}-09$ & 2.50 & 1.71 & 0.40 \\
\hline $\mathrm{N}_{2} \mathrm{O}$ & $1.9 \mathrm{E}-09$ & $1.3 \mathrm{E}-09$ & $2.9 \mathrm{E}-11$ & $5.1 \mathrm{E}-10$ & $6.1 \mathrm{E}-10$ & $7.0 \mathrm{E}-11$ & $7.3 \mathrm{E}-10$ & 2.81 & 1.98 & 0.45 \\
\hline $\mathrm{CN}$ & $5.2 \mathrm{E}-10$ & $4.3 \mathrm{E}-10$ & $2.3 \mathrm{E}-14$ & $5.1 \mathrm{E}-11$ & $2.1 \mathrm{E}-11$ & $1.6 \mathrm{E}-11$ & $1.3 \mathrm{E}-10$ & 3.50 & 2.55 & 0.45 \\
\hline $\mathrm{HCN}$ & $1.6 \mathrm{E}-09$ & $1.2 \mathrm{E}-09$ & $5.7 \mathrm{E}-12$ & $2.8 \mathrm{E}-10$ & $5.3 \mathrm{E}-09$ & $2.1 \mathrm{E}-10$ & $3.3 \mathrm{E}-09$ & 3.12 & 2.23 & 0.45 \\
\hline $\mathrm{HC}_{3} \mathrm{~N}$ & $7.1 \mathrm{E}-09$ & $4.7 \mathrm{E}-09$ & $6.2 \mathrm{E}-10$ & $3.5 \mathrm{E}-09$ & $3.1 \mathrm{E}-09$ & $9.6 \mathrm{E}-10$ & $3.8 \mathrm{E}-09$ & 2.59 & 1.79 & 0.45 \\
\hline $\mathrm{CH}_{3} \mathrm{OH}$ & $1.4 \mathrm{E}-09$ & $9.5 \mathrm{E}-10$ & $1.2 \mathrm{E}-10$ & $5.3 \mathrm{E}-10$ & $2.2 \mathrm{E}-09$ & $2.1 \mathrm{E}-10$ & $1.7 \mathrm{E}-09$ & 2.76 & 1.92 & 0.44 \\
\hline $\mathrm{CH}_{3} \mathrm{CN}$ & $3.0 \mathrm{E}-09$ & $2.1 \mathrm{E}-09$ & $9.1 \mathrm{E}-12$ & $5.4 \mathrm{E}-10$ & $1.7 \mathrm{E}-09$ & $1.1 \mathrm{E}-10$ & $1.6 \mathrm{E}-09$ & 3.07 & 2.18 & 0.45 \\
\hline $\mathrm{CH}_{3} \mathrm{SH}$ & $2.8 \mathrm{E}-09$ & $1.8 \mathrm{E}-09$ & $7.4 \mathrm{E}-09$ & $2.8 \mathrm{E}-09$ & $3.4 \mathrm{E}-09$ & $8.5 \mathrm{E}-09$ & $3.1 \mathrm{E}-09$ & 2.50 & 1.72 & 0.39 \\
\hline
\end{tabular}

Notes. In units of $\mathrm{s}^{-1}$. These rates are for unshielded atoms and molecules exposed to the full three-dimensional interstellar radiation field, with various wavelength dependences described in Sect. 2. Dust shielding functions, $\theta$, for an infinite-slab interstellar cloud are fit to functions of the visual extinction, $A_{\mathrm{V}}$, according to two formulae: $\theta\left(A_{\mathrm{V}}\right)=\exp \left(-\gamma_{\exp } A_{\mathrm{V}}\right)$ and $\theta\left(A_{\mathrm{V}}\right)=E_{2}\left(\gamma_{\mathrm{E}_{2}} A_{\mathrm{V}}\right)$ (where $E_{2}$ is the 2nd-order exponential integral), both assuming incident radiation at the cloud edge with the wavelength dependence of our standard ISRF radiation field (Eqs. (12) and (13) in Sect. 6.2). Values for $\gamma_{\mathrm{E}_{2}}$ are given assuming an interstellar dust size distribution (ISM), and following the growth of dust grains in a protoplanetary disk (growth), as described in Sect. 8.1. 
Table 18. continued.

\begin{tabular}{|c|c|c|c|c|c|c|c|c|c|c|}
\hline \multirow[b]{2}{*}{ Species } & \multirow[b]{2}{*}{ ISRF } & \multirow[b]{2}{*}{ Mathis '83 } & \multirow[b]{2}{*}{$4000 \mathrm{~K}$} & \multirow[b]{2}{*}{$10000 \mathrm{~K}$} & \multirow[b]{2}{*}{ Lyman- $\alpha$} & \multirow[b]{2}{*}{ Solar } & \multirow[b]{2}{*}{ TW-Hydra } & \multicolumn{3}{|c|}{ ISRF dust shielding } \\
\hline & & & & & & & & $\gamma_{\text {exp,ISM }}$ & $\gamma_{\mathrm{E}_{2}, \mathrm{ISM}}$ & $\gamma_{\mathrm{E}_{2}, \text { growth }}$ \\
\hline $\mathrm{CH}_{3} \mathrm{CHO}$ & $2.0 \mathrm{E}-09$ & $1.3 \mathrm{E}-09$ & $3.8 \mathrm{E}-09$ & $1.6 \mathrm{E}-09$ & $3.2 \mathrm{E}-09$ & $4.8 \mathrm{E}-09$ & $2.7 \mathrm{E}-09$ & 2.46 & 1.69 & 0.41 \\
\hline $\mathrm{CH}_{3} \mathrm{NH}_{2}$ & $7.3 \mathrm{E}-10$ & $4.8 \mathrm{E}-10$ & $4.2 \mathrm{E}-09$ & $1.3 \mathrm{E}-09$ & $1.0 \mathrm{E}-15$ & $6.3 \mathrm{E}-09$ & $4.3 \mathrm{E}-10$ & 2.37 & 1.62 & 0.34 \\
\hline $\mathrm{NH}_{2} \mathrm{CHO}$ & $2.7 \mathrm{E}-09$ & $1.8 \mathrm{E}-09$ & $2.3 \mathrm{E}-09$ & $3.1 \mathrm{E}-09$ & $2.9 \mathrm{E}-09$ & $2.7 \mathrm{E}-09$ & $3.0 \mathrm{E}-09$ & 2.40 & 1.64 & 0.37 \\
\hline $\mathrm{C}_{2} \mathrm{H}_{5} \mathrm{OH}$ & $2.5 \mathrm{E}-09$ & $1.7 \mathrm{E}-09$ & $2.1 \mathrm{E}-10$ & $9.2 \mathrm{E}-10$ & $4.0 \mathrm{E}-09$ & $3.7 \mathrm{E}-10$ & $3.0 \mathrm{E}-09$ & 2.77 & 1.93 & 0.44 \\
\hline $\mathrm{C}_{3} \mathrm{H}_{7} \mathrm{OH}$ & $4.0 \mathrm{E}-09$ & $2.7 \mathrm{E}-09$ & $3.8 \mathrm{E}-10$ & $1.5 \mathrm{E}-09$ & $8.1 \mathrm{E}-09$ & $6.8 \mathrm{E}-10$ & $5.8 \mathrm{E}-09$ & 2.76 & 1.92 & 0.44 \\
\hline SH & $1.2 \mathrm{E}-09$ & $8.1 \mathrm{E}-10$ & $3.4 \mathrm{E}-08$ & $2.8 \mathrm{E}-09$ & $1.2 \mathrm{E}-09$ & $4.9 \mathrm{E}-08$ & $1.6 \mathrm{E}-09$ & 2.40 & 1.64 & 0.32 \\
\hline $\mathrm{SH}^{+}$ & $6.9 \mathrm{E}-10$ & $5.2 \mathrm{E}-10$ & $4.0 \mathrm{E}-08$ & $3.2 \mathrm{E}-10$ & $1.2 \mathrm{E}-11$ & $4.6 \mathrm{E}-08$ & $2.4 \mathrm{E}-10$ & 2.83 & 1.79 & 0.40 \\
\hline $\mathrm{H}_{2} \mathrm{~S}$ & $3.1 \mathrm{E}-09$ & $2.1 \mathrm{E}-09$ & $4.2 \mathrm{E}-09$ & $2.2 \mathrm{E}-09$ & $8.4 \mathrm{E}-09$ & $6.1 \mathrm{E}-09$ & $4.4 \mathrm{E}-09$ & 2.64 & 1.83 & 0.41 \\
\hline $\mathrm{CS}$ & $9.5 \mathrm{E}-10$ & $6.3 \mathrm{E}-10$ & $5.2 \mathrm{E}-12$ & $2.9 \mathrm{E}-10$ & $8.4 \mathrm{E}-09$ & $3.1 \mathrm{E}-10$ & $4.9 \mathrm{E}-09$ & 2.77 & 1.95 & 0.45 \\
\hline $\mathrm{CS}_{2}$ & $8.8 \mathrm{E}-09$ & $6.2 \mathrm{E}-09$ & $5.0 \mathrm{E}-08$ & $1.9 \mathrm{E}-08$ & $2.7 \mathrm{E}-09$ & $5.1 \mathrm{E}-08$ & $6.7 \mathrm{E}-09$ & 2.50 & 1.72 & 0.32 \\
\hline $\mathrm{OCS}^{2}$ & $4.7 \mathrm{E}-09$ & $3.1 \mathrm{E}-09$ & $1.6 \mathrm{E}-09$ & $3.8 \mathrm{E}-09$ & $1.1 \mathrm{E}-09$ & $1.8 \mathrm{E}-09$ & $3.7 \mathrm{E}-09$ & 2.46 & 1.68 & 0.42 \\
\hline $\mathrm{S}_{2}$ & $6.6 \mathrm{E}-10$ & $3.7 \mathrm{E}-10$ & $2.0 \mathrm{E}-07$ & 4.3E-09 & - & $2.1 \mathrm{E}-07$ & $1.0 \mathrm{E}-09$ & 1.90 & 1.28 & 0.21 \\
\hline SO & $4.2 \mathrm{E}-09$ & $2.9 \mathrm{E}-09$ & $8.4 \mathrm{E}-09$ & $3.0 \mathrm{E}-09$ & $1.7 \mathrm{E}-08$ & $1.2 \mathrm{E}-08$ & $1.1 \mathrm{E}-08$ & 2.76 & 1.94 & 0.40 \\
\hline $\mathrm{SO}_{2}$ & $2.4 \mathrm{E}-09$ & $1.7 \mathrm{E}-09$ & $3.6 \mathrm{E}-09$ & $1.6 \mathrm{E}-09$ & $6.1 \mathrm{E}-09$ & $5.1 \mathrm{E}-09$ & 4.3E-09 & 2.78 & 1.94 & 0.40 \\
\hline $\mathrm{SiH}^{2}$ & $2.7 \mathrm{E}-09$ & $1.8 \mathrm{E}-09$ & $1.1 \mathrm{E}-06$ & $1.5 \mathrm{E}-08$ & $7.9 \mathrm{E}-12$ & $1.0 \mathrm{E}-06$ & $5.2 \mathrm{E}-09$ & 1.95 & 1.24 & 0.23 \\
\hline $\mathrm{SiH}^{+}$ & $2.6 \mathrm{E}-09$ & $2.6 \mathrm{E}-09$ & $5.4 \mathrm{E}-06$ & $1.1 \mathrm{E}-08$ & $3.5 \mathrm{E}-09$ & $4.1 \mathrm{E}-06$ & $1.0 \mathrm{E}-08$ & 1.55 & 0.94 & 0.24 \\
\hline $\mathrm{SiO}$ & $1.6 \mathrm{E}-09$ & $1.0 \mathrm{E}-09$ & $1.4 \mathrm{E}-11$ & $6.1 \mathrm{E}-10$ & $7.9 \mathrm{E}-12$ & $1.5 \mathrm{E}-11$ & $3.5 \mathrm{E}-10$ & 2.66 & 1.85 & 0.45 \\
\hline $\mathrm{HCl}$ & $1.7 \mathrm{E}-09$ & $1.2 \mathrm{E}-09$ & $9.4 \mathrm{E}-11$ & $5.1 \mathrm{E}-10$ & $1.5 \mathrm{E}-10$ & $1.1 \mathrm{E}-10$ & $5.6 \mathrm{E}-10$ & 2.88 & 2.02 & 0.44 \\
\hline $\mathrm{HCl}^{+}$ & $1.1 \mathrm{E}-10$ & $8.5 \mathrm{E}-11$ & $1.0 \mathrm{E}-12$ & $2.4 \mathrm{E}-11$ & $1.3 \mathrm{E}-10$ & $7.9 \mathrm{E}-12$ & $1.0 \mathrm{E}-10$ & 3.01 & 2.12 & 0.45 \\
\hline $\mathrm{NaCl}$ & $9.5 \mathrm{E}-10$ & $6.0 \mathrm{E}-10$ & $6.0 \mathrm{E}-08$ & $3.6 \mathrm{E}-09$ & $7.9 \mathrm{E}-12$ & $6.1 \mathrm{E}-08$ & $8.4 \mathrm{E}-10$ & 2.20 & 1.50 & 0.26 \\
\hline $\mathrm{PH}$ & $5.8 \mathrm{E}-10$ & $3.8 \mathrm{E}-10$ & $6.8 \mathrm{E}-11$ & $4.2 \mathrm{E}-10$ & $1.9 \mathrm{E}-11$ & $5.9 \mathrm{E}-11$ & $3.2 \mathrm{E}-10$ & 2.48 & 1.71 & 0.43 \\
\hline $\mathrm{PH}^{+}$ & $1.4 \mathrm{E}-10$ & $1.1 \mathrm{E}-10$ & $9.6 \mathrm{E}-08$ & $4.1 \mathrm{E}-10$ & $1.7 \mathrm{E}-10$ & $8.0 \mathrm{E}-08$ & $3.1 \mathrm{E}-10$ & 1.93 & 1.16 & 0.28 \\
\hline $\mathrm{AlH}$ & $2.6 \mathrm{E}-10$ & $1.9 \mathrm{E}-10$ & $4.0 \mathrm{E}-09$ & $1.1 \mathrm{E}-09$ & - & $5.3 \mathrm{E}-09$ & $2.3 \mathrm{E}-10$ & 2.42 & 1.67 & 0.26 \\
\hline $\mathrm{LiH}$ & $4.9 \mathrm{E}-09$ & $3.0 \mathrm{E}-09$ & $2.1 \mathrm{E}-06$ & $3.3 \mathrm{E}-08$ & $7.9 \mathrm{E}-12$ & $2.2 \mathrm{E}-06$ & $8.4 \mathrm{E}-09$ & 1.81 & 1.21 & 0.21 \\
\hline $\mathrm{MgH}$ & $5.1 \mathrm{E}-10$ & $3.3 \mathrm{E}-10$ & $2.4 \mathrm{E}-08$ & $8.8 \mathrm{E}-10$ & $7.9 \mathrm{E}-12$ & $3.2 \mathrm{E}-08$ & $2.7 \mathrm{E}-10$ & 2.30 & 1.54 & 0.36 \\
\hline $\mathrm{NaH}$ & $7.0 \mathrm{E}-09$ & $4.5 \mathrm{E}-09$ & $3.7 \mathrm{E}-06$ & $4.6 \mathrm{E}-08$ & $7.9 \mathrm{E}-12$ & $4.0 \mathrm{E}-06$ & $1.3 \mathrm{E}-08$ & 1.77 & 1.17 & 0.21 \\
\hline
\end{tabular}

orders of magnitude. With this normalisation, molecules with relatively long-wavelength photodissociation thresholds show massively increased rates when irradiated by a $4000 \mathrm{~K}$ black body and sometimes supersede the scales of our figures by orders of magnitude. This effect is illustrated in Fig. 21 for four molecules with successively shorter-wavelength dissociation thresholds, in the order $\mathrm{SiH}^{+}, \mathrm{NH}_{3}, \mathrm{HC}_{3} \mathrm{~N}$, and $\mathrm{N}_{2}$, and with respective ratios between $4000 \mathrm{~K}$ and ISRF photodissociation rates of $1800,2.5,0.09,2 \times 10^{-6}$. The rapid increase of black body intensity longwards of $170 \mathrm{~nm}$ is responsible for this variation. The $4000 \mathrm{~K}$ photodissociation of $\mathrm{CH}_{3}$ and $\mathrm{NH}_{3}$ is dominated by absorption longer than $170 \mathrm{~nm}$ into their lowestlying electronic states. In contrast, the very-short wavelength threshold of $\mathrm{N}_{2}$ makes it completely immune to $4000 \mathrm{~K}$ radiation. Figure 22 illustrates this point further by plotting the photodissociation or ionisation thresholds of all species versus their $4000 \mathrm{~K}$ and ISRF rate ratios, showing a sharp drop shortwards of $170 \mathrm{~nm}$. According to this figure, these ratios vary by more than an order of magnitude due to other details of each atomic or molecular cross section.

Figures 19 and 20 also show rates assuming a Lyman- $\alpha$ dominated radiation field, most of which are smaller than in the ISRF case (under our normalisation scheme) or zero if there is no significant cross section at $121.6 \mathrm{~nm}$. Most molecules and atoms have photoionisation thresholds at similar or shorter wavelengths than this (as listed in Table 1), explaining the general disappearance or lowering of photoionisation rates for Lyman- $\alpha$ radiation.

For molecules with line-dominated cross sections the Lyman- $\alpha$ cross section is sensitive to the positions of these lines. For example, Fig. 23 shows why the photodissociation rate of $\mathrm{C}_{2} \mathrm{H}_{2}$ increases by a factor of three when substituting the ISRF with a Lyman- $\alpha$ emission line, while $\mathrm{HC}_{3} \mathrm{~N}$ decreases by a factor 2. In this case the respective overlap and non-overlap of resonances is responsible.

\subsection{Comparison with previous rates}

The new ISRF photodissociation and ionisation rates are compared with those calculated from cross sections taken from the previous version of the Leiden database (van Dishoeck 1988; van Dishoeck et al. 2006) and the PHIDRATES database (Huebner et al. 1992; Huebner \& Mukherjee 2015) in Fig. 24. Not all species are included in all databases. We generally find agreement within $30 \%$ with the molecular rates from the previous Leiden database with exceptional cases being the photodissociation of $\mathrm{CH}_{3}, \mathrm{HCl}$ and the photoionisation of $\mathrm{C}_{2} \mathrm{H}_{2}$. All of these processes have larger rates in the updated database, by factors between 1.6 and 2.3, and this is due to the availability of new theoretical and experimental data, especially at shorter wavelengths, leading to increased cross sections.

More disagreement follows from comparison with the PHIDRATES database, with differences spanning factors of 0.15 to 3.8, and the extreme case of $\mathrm{CH}_{3} \mathrm{CHO}$ photodissociation for which the PHIDRATES cross section is based on somewhat incomplete information. In general PHIDRATES molecular cross sections were collated originally by Huebner et al. (1992) and in previous studies, and the subsequent availability of new experimental and theoretical information explains the different ISRF photodestruction rates.

It is important to note the differing intentions of the Leiden and PHIDRATES databases. The former considers cross sections important to interstellar chemistry, so that metastable dissociation products generally have time to decay before they can react, and the Lyman-limit at $91.2 \mathrm{~nm}$ provides a hard 
A. N. Heays et al.: Photodissociation and photoionisation of atoms and molecules of astrophysical interest

Table 19. Photoionisation rates of atoms and molecules and parameterised dust.

\begin{tabular}{|c|c|c|c|c|c|c|c|c|c|c|}
\hline \multirow[b]{2}{*}{ Species } & \multirow[b]{2}{*}{ ISRF } & \multirow[b]{2}{*}{ Mathis ' 83} & \multirow[b]{2}{*}{$4000 \mathrm{~K}$} & \multirow[b]{2}{*}{$10000 \mathrm{~K}$} & \multirow[b]{2}{*}{ Lyman- $\alpha$} & \multirow[b]{2}{*}{ Solar } & \multirow[b]{2}{*}{ TW-Hydra } & \multicolumn{3}{|c|}{ ISRF dust shielding } \\
\hline & & & & & & & & $\gamma_{\text {exp,ISM }}$ & $\gamma_{\mathrm{E}_{2}, \mathrm{ISM}}$ & $\gamma_{\mathrm{E}_{2}, \text { growth }}$ \\
\hline $\mathrm{H}$ & - & - & - & - & - & $1.6 \mathrm{E}-12$ & $5.4 \mathrm{E}-12$ & 3.00 & - & - \\
\hline $\mathrm{Li}$ & $3.4 \mathrm{E}-10$ & $2.3 \mathrm{E}-10$ & $3.1 \mathrm{E}-09$ & $7.1 \mathrm{E}-10$ & $2.1 \mathrm{E}-10$ & $4.9 \mathrm{E}-09$ & $3.3 \mathrm{E}-10$ & 2.45 & 1.68 & 0.32 \\
\hline $\mathrm{C}$ & $3.5 \mathrm{E}-10$ & $2.6 \mathrm{E}-10$ & $5.7 \mathrm{E}-15$ & $2.8 \mathrm{E}-11$ & - & $1.0 \mathrm{E}-11$ & $8.3 \mathrm{E}-11$ & 3.76 & 2.77 & 0.45 \\
\hline $\mathrm{N}$ & - & - & - & - & - & $1.6 \mathrm{E}-12$ & $7.8 \mathrm{E}-12$ & - & - & - \\
\hline $\mathrm{O}$ & - & - & - & - & - & $1.4 \mathrm{E}-12$ & $6.2 \mathrm{E}-12$ & - & - & - \\
\hline $\mathrm{Na}$ & $1.4 \mathrm{E}-11$ & $9.1 \mathrm{E}-12$ & $1.3 \mathrm{E}-10$ & $1.5 \mathrm{E}-11$ & $1.7 \mathrm{E}-11$ & $1.4 \mathrm{E}-10$ & $1.5 \mathrm{E}-11$ & 2.62 & 1.81 & 0.37 \\
\hline $\mathrm{Mg}$ & $6.6 \mathrm{E}-11$ & $4.3 \mathrm{E}-11$ & $8.1 \mathrm{E}-12$ & $5.3 \mathrm{E}-11$ & $1.1 \mathrm{E}-11$ & $7.3 \mathrm{E}-12$ & $4.2 \mathrm{E}-11$ & 2.43 & 1.67 & 0.43 \\
\hline $\mathrm{Al}$ & $4.4 \mathrm{E}-09$ & $3.0 \mathrm{E}-09$ & $2.1 \mathrm{E}-08$ & $1.0 \mathrm{E}-08$ & $1.4 \mathrm{E}-09$ & $2.1 \mathrm{E}-08$ & $3.7 \mathrm{E}-09$ & 2.36 & 1.62 & 0.31 \\
\hline $\mathrm{Si}$ & $4.5 \mathrm{E}-09$ & $2.9 \mathrm{E}-09$ & $9.3 \mathrm{E}-11$ & $2.0 \mathrm{E}-09$ & $5.7 \mathrm{E}-09$ & $2.9 \mathrm{E}-10$ & $4.4 \mathrm{E}-09$ & 2.61 & 1.81 & 0.45 \\
\hline $\mathrm{P}$ & $1.9 \mathrm{E}-09$ & $1.4 \mathrm{E}-09$ & $1.4 \mathrm{E}-13$ & $2.1 \mathrm{E}-10$ & - & $3.3 \mathrm{E}-11$ & $3.6 \mathrm{E}-10$ & 3.45 & 2.51 & 0.45 \\
\hline $\mathrm{S}$ & $1.1 \mathrm{E}-09$ & $8.5 \mathrm{E}-10$ & $8.3 \mathrm{E}-14$ & $1.2 \mathrm{E}-10$ & $1.1 \mathrm{E}-16$ & $2.4 \mathrm{E}-11$ & $2.3 \mathrm{E}-10$ & 3.52 & 2.57 & 0.45 \\
\hline $\mathrm{Cl}$ & $4.7 \mathrm{E}-11$ & $5.5 \mathrm{E}-11$ & - & $2.5 \mathrm{E}-12$ & - & $1.1 \mathrm{E}-11$ & $4.9 \mathrm{E}-11$ & 4.30 & 3.21 & 0.45 \\
\hline $\mathrm{K}$ & $3.9 \mathrm{E}-11$ & $2.6 \mathrm{E}-11$ & $3.7 \mathrm{E}-10$ & $5.9 \mathrm{E}-11$ & $3.5 \mathrm{E}-11$ & $4.2 \mathrm{E}-10$ & $4.0 \mathrm{E}-11$ & 2.48 & 1.70 & 0.35 \\
\hline $\mathrm{Ca}$ & $3.5 \mathrm{E}-10$ & $2.3 \mathrm{E}-10$ & $8.0 \mathrm{E}-10$ & $6.2 \mathrm{E}-10$ & $1.4 \mathrm{E}-10$ & $7.9 \mathrm{E}-10$ & $2.9 \mathrm{E}-10$ & 2.34 & 1.60 & 0.34 \\
\hline $\mathrm{Ti}$ & $2.4 \mathrm{E}-10$ & $1.6 \mathrm{E}-10$ & $9.8 \mathrm{E}-12$ & $7.7 \mathrm{E}-11$ & $4.2 \mathrm{E}-10$ & $2.6 \mathrm{E}-11$ & $2.9 \mathrm{E}-10$ & 2.81 & 1.96 & 0.44 \\
\hline $\mathrm{Cr}$ & $1.6 \mathrm{E}-09$ & $1.1 \mathrm{E}-09$ & $1.7 \mathrm{E}-09$ & $2.1 \mathrm{E}-09$ & $1.2 \mathrm{E}-09$ & $1.9 \mathrm{E}-09$ & $1.4 \mathrm{E}-09$ & 2.39 & 1.63 & 0.35 \\
\hline Mn & $3.3 \mathrm{E}-11$ & $2.2 \mathrm{E}-11$ & $6.8 \mathrm{E}-12$ & $3.4 \mathrm{E}-11$ & $7.0 \mathrm{E}-12$ & $5.8 \mathrm{E}-12$ & $2.6 \mathrm{E}-11$ & 2.35 & 1.61 & 0.41 \\
\hline $\mathrm{Fe}$ & $4.7 \mathrm{E}-10$ & $3.1 \mathrm{E}-10$ & $1.2 \mathrm{E}-11$ & $2.1 \mathrm{E}-10$ & $7.7 \mathrm{E}-10$ & $3.9 \mathrm{E}-11$ & $5.9 \mathrm{E}-10$ & 2.62 & 1.81 & 0.45 \\
\hline $\mathrm{Co}$ & $5.3 \mathrm{E}-11$ & $3.4 \mathrm{E}-11$ & $3.3 \mathrm{E}-12$ & $3.7 \mathrm{E}-11$ & $2.8 \mathrm{E}-11$ & $3.5 \mathrm{E}-12$ & $4.5 \mathrm{E}-11$ & 2.47 & 1.70 & 0.45 \\
\hline $\mathrm{Ni}$ & $9.8 \mathrm{E}-11$ & $6.3 \mathrm{E}-11$ & $9.5 \mathrm{E}-12$ & $7.5 \mathrm{E}-11$ & $4.9 \mathrm{E}-11$ & $9.5 \mathrm{E}-12$ & $7.7 \mathrm{E}-11$ & 2.43 & 1.67 & 0.44 \\
\hline $\mathrm{Zn}$ & $4.1 \mathrm{E}-10$ & $2.9 \mathrm{E}-10$ & $3.1 \mathrm{E}-14$ & $3.8 \mathrm{E}-11$ & $9.2 \mathrm{E}-12$ & $2.9 \mathrm{E}-12$ & $6.6 \mathrm{E}-11$ & 3.25 & 2.35 & 0.45 \\
\hline $\mathrm{Rb}$ & $2.7 \mathrm{E}-11$ & $1.8 \mathrm{E}-11$ & $1.6 \mathrm{E}-09$ & $4.8 \mathrm{E}-11$ & $2.3 \mathrm{E}-11$ & $2.2 \mathrm{E}-09$ & $3.0 \mathrm{E}-11$ & 2.33 & 1.54 & 0.34 \\
\hline $\mathrm{Ca}^{+}$ & $2.4 \mathrm{E}-12$ & $2.0 \mathrm{E}-12$ & - & $1.7 \mathrm{E}-13$ & - & $5.2 \mathrm{E}-14$ & $6.0 \mathrm{E}-13$ & 4.09 & 3.04 & 0.45 \\
\hline $\mathrm{H}^{-}$ & $1.5 \mathrm{E}-07$ & $1.6 \mathrm{E}-08$ & $1.6 \mathrm{E}-03$ & $2.5 \mathrm{E}-07$ & $7.5 \mathrm{E}-10$ & $1.4 \mathrm{E}-05$ & $2.3 \mathrm{E}-06$ & 1.24 & 0.74 & 0.22 \\
\hline $\mathrm{H}_{2}$ & - & - & - & - & - & $4.9 \mathrm{E}-13$ & $3.2 \mathrm{E}-12$ & - & - & - \\
\hline $\mathrm{CH}$ & $7.6 \mathrm{E}-10$ & $5.6 \mathrm{E}-10$ & $2.3 \mathrm{E}-14$ & $6.8 \mathrm{E}-11$ & - & $9.6 \mathrm{E}-12$ & $1.4 \mathrm{E}-10$ & 3.67 & 2.70 & 0.45 \\
\hline $\mathrm{CH}_{3}$ & $3.3 \mathrm{E}-10$ & $2.3 \mathrm{E}-10$ & $8.6 \mathrm{E}-14$ & $4.5 \mathrm{E}-11$ & $8.4 \mathrm{E}-10$ & $3.6 \mathrm{E}-11$ & $5.5 \mathrm{E}-10$ & 3.26 & 2.36 & 0.45 \\
\hline $\mathrm{CH}_{4}$ & $1.0 \mathrm{E}-11$ & $1.2 \mathrm{E}-11$ & - & $5.5 \mathrm{E}-13$ & - & $9.3 \mathrm{E}-12$ & $3.8 \mathrm{E}-11$ & 4.31 & 3.21 & 0.45 \\
\hline $\mathrm{C}_{2}$ & $4.1 \mathrm{E}-10$ & $3.4 \mathrm{E}-10$ & $1.4 \mathrm{E}-15$ & $2.7 \mathrm{E}-11$ & - & $6.7 \mathrm{E}-12$ & $8.7 \mathrm{E}-11$ & 4.19 & 3.12 & 0.45 \\
\hline $\mathrm{C}_{2} \mathrm{H}_{2}$ & $5.3 \mathrm{E}-10$ & $4.1 \mathrm{E}-10$ & $5.2 \mathrm{E}-15$ & $4.0 \mathrm{E}-11$ & - & $2.4 \mathrm{E}-11$ & $1.7 \mathrm{E}-10$ & 3.92 & 2.91 & 0.45 \\
\hline $\mathrm{C}_{2} \mathrm{H}_{4}^{2}$ & $4.1 \mathrm{E}-10$ & $3.2 \mathrm{E}-10$ & $1.7 \mathrm{E}-14$ & $3.7 \mathrm{E}-11$ & - & $6.2 \mathrm{E}-12$ & $8.1 \mathrm{E}-11$ & 3.63 & 2.67 & 0.45 \\
\hline $\mathrm{C}_{2} \mathrm{H}_{6}$ & $2.3 \mathrm{E}-10$ & $2.0 \mathrm{E}-10$ & $7.9 \mathrm{E}-16$ & $1.5 \mathrm{E}-11$ & - & $2.3 \mathrm{E}-11$ & $1.2 \mathrm{E}-10$ & 4.17 & 3.10 & 0.45 \\
\hline $\mathrm{C}_{3}$ & $1.4 \mathrm{E}-10$ & $1.1 \mathrm{E}-10$ & $9.0 \mathrm{E}-16$ & $9.7 \mathrm{E}-12$ & - & $3.5 \mathrm{E}-12$ & $3.3 \mathrm{E}-11$ & 4.03 & 3.00 & 0.45 \\
\hline $\mathrm{H}_{2} \mathrm{O}$ & $2.7 \mathrm{E}-11$ & $2.6 \mathrm{E}-11$ & - & $1.7 \mathrm{E}-12$ & - & $4.2 \mathrm{E}-12$ & $2.2 \mathrm{E}-11$ & 4.27 & 3.18 & 0.45 \\
\hline $\mathrm{O}_{2}$ & $5.1 \mathrm{E}-11$ & $4.5 \mathrm{E}-11$ & $1.3 \mathrm{E}-16$ & $3.4 \mathrm{E}-12$ & - & $3.4 \mathrm{E}-12$ & $2.2 \mathrm{E}-11$ & 4.22 & 3.14 & 0.45 \\
\hline $\mathrm{H}_{2} \mathrm{O}_{2}$ & $2.5 \mathrm{E}-10$ & $2.0 \mathrm{E}-10$ & $3.2 \mathrm{E}-15$ & $1.9 \mathrm{E}-11$ & - & $1.2 \mathrm{E}-11$ & $7.7 \mathrm{E}-11$ & 3.88 & 2.88 & 0.45 \\
\hline $\mathrm{O}_{3}{ }^{2}$ & $3.3 \mathrm{E}-11$ & $3.4 \mathrm{E}-11$ & - & $2.1 \mathrm{E}-12$ & - & $7.5 \mathrm{E}-12$ & $3.5 \mathrm{E}-11$ & 4.28 & 3.19 & 0.45 \\
\hline $\mathrm{CO}$ & - & - & - & - & - & $6.5 \mathrm{E}-12$ & $2.5 \mathrm{E}-11$ & - & - & - \\
\hline $\mathrm{CO}_{2}$ & - & - & - & - & - & $5.2 \mathrm{E}-12$ & $2.2 \mathrm{E}-11$ & - & - & - \\
\hline $\mathrm{H}_{2} \mathrm{CO}$ & $4.0 \mathrm{E}-10$ & $3.1 \mathrm{E}-10$ & $1.1 \mathrm{E}-14$ & $3.5 \mathrm{E}-11$ & - & $1.3 \mathrm{E}-11$ & $1.1 \mathrm{E}-10$ & 3.66 & 2.69 & 0.45 \\
\hline NH & $1.9 \mathrm{E}-12$ & $3.0 \mathrm{E}-12$ & - & - & - & $2.8 \mathrm{E}-12$ & $1.0 \mathrm{E}-11$ & 4.34 & 3.24 & 0.45 \\
\hline $\mathrm{NH}_{2}$ & $1.9 \mathrm{E}-10$ & $1.5 \mathrm{E}-10$ & $1.7 \mathrm{E}-15$ & $1.4 \mathrm{E}-11$ & - & $8.7 \mathrm{E}-12$ & $5.8 \mathrm{E}-11$ & 3.97 & 2.94 & 0.45 \\
\hline $\mathrm{NH}_{3}^{2}$ & $2.7 \mathrm{E}-10$ & $2.0 \mathrm{E}-10$ & $1.7 \mathrm{E}-14$ & $2.8 \mathrm{E}-11$ & $4.8 \mathrm{E}-11$ & $9.6 \mathrm{E}-12$ & $9.0 \mathrm{E}-11$ & 3.49 & 2.54 & 0.45 \\
\hline $\mathrm{N}_{2}^{3}$ & - & - & - & - & - & $1.3 \mathrm{E}-12$ & $8.8 \mathrm{E}-12$ & - & - & - \\
\hline $\mathrm{NO}$ & $2.6 \mathrm{E}-10$ & $1.9 \mathrm{E}-10$ & $7.4 \mathrm{E}-14$ & $3.1 \mathrm{E}-11$ & $3.3 \mathrm{E}-10$ & $1.9 \mathrm{E}-11$ & $2.6 \mathrm{E}-10$ & 3.38 & 2.46 & 0.45 \\
\hline $\mathrm{NO}_{2}$ & $1.5 \mathrm{E}-10$ & $1.2 \mathrm{E}-10$ & $4.2 \mathrm{E}-15$ & $1.3 \mathrm{E}-11$ & $1.7 \mathrm{E}-11$ & $3.1 \mathrm{E}-12$ & $4.1 \mathrm{E}-11$ & 3.75 & 2.77 & 0.45 \\
\hline $\mathrm{N}_{2} \mathrm{O}$ & $1.7 \mathrm{E}-10$ & $1.9 \mathrm{E}-10$ & $1.8 \mathrm{E}-16$ & $1.0 \mathrm{E}-11$ & - & $3.8 \mathrm{E}-12$ & $3.7 \mathrm{E}-11$ & 4.30 & 3.20 & 0.45 \\
\hline $\mathrm{CN}$ & - & - & - & - & - & $2.0 \mathrm{E}-12$ & $5.9 \mathrm{E}-12$ & - & - & - \\
\hline $\mathrm{HCN}$ & $4.4 \mathrm{E}-13$ & $7.0 \mathrm{E}-13$ & - & $4.2 \mathrm{E}-15$ & - & $7.5 \mathrm{E}-12$ & $2.8 \mathrm{E}-11$ & 4.34 & 3.24 & 0.45 \\
\hline $\mathrm{HC}_{3} \mathrm{~N}$ & $2.3 \mathrm{E}-10$ & $1.8 \mathrm{E}-10$ & $1.3 \mathrm{E}-15$ & $1.6 \mathrm{E}-11$ & - & $1.2 \mathrm{E}-11$ & $7.7 \mathrm{E}-11$ & 4.07 & 3.02 & 0.45 \\
\hline
\end{tabular}

Notes. In units of $\mathrm{s}^{-1}$. These rates are for unshielded atoms and molecules exposed to the full three-dimensional interstellar radiation field, with various wavelength dependences described in Sect. 2. Dust shielding functions, $\theta$, for an infinite-slab interstellar cloud are fit to functions of the visual extinction, $A_{\mathrm{V}}$, according to two formulae: $\theta\left(A_{\mathrm{V}}\right)=\exp \left(-\gamma_{\exp } A_{\mathrm{V}}\right)$ and $\theta\left(A_{\mathrm{V}}\right)=E_{2}\left(\gamma_{\mathrm{E}_{2}} A_{\mathrm{V}}\right)$ (where $E_{2}$ is the 2nd-order exponential integral), both assuming incident radiation at the cloud edge with the wavelength dependence of our standard ISRF radiation field (Eqs. (12) and (13) in Sect. 6.2). Values for $\gamma_{\mathrm{E}_{2}}$ are given assuming an interstellar dust size distribution (ISM), and following the growth of dust grains in a protoplanetary disk (growth), as described in Sect. 8.1. 
Table 19. continued.

\begin{tabular}{ccccccccccc}
\hline \hline & & & & & & \multicolumn{4}{c}{ ISRF dust shielding } \\
Species & ISRF & Mathis '83 & $4000 \mathrm{~K}$ & $10000 \mathrm{~K}$ & Lyman- $\alpha$ & Solar & TW-Hydra & $\gamma_{\text {exp,ISM }}$ & $\gamma_{\mathrm{E}_{2} \text {,ISM }}$ & $\gamma_{\mathrm{E}_{2}, \text { growth }}$ \\
\hline $\mathrm{CH}_{3} \mathrm{OH}$ & $3.1 \mathrm{E}-10$ & $2.5 \mathrm{E}-10$ & $5.5 \mathrm{E}-15$ & $2.5 \mathrm{E}-11$ & - & $1.6 \mathrm{E}-11$ & $1.1 \mathrm{E}-10$ & 3.78 & 2.80 & 0.45 \\
$\mathrm{CH}_{3} \mathrm{CN}$ & $1.2 \mathrm{E}-10$ & $1.1 \mathrm{E}-10$ & $3.8 \mathrm{E}-16$ & $7.8 \mathrm{E}-12$ & - & $1.6 \mathrm{E}-11$ & $7.7 \mathrm{E}-11$ & 4.19 & 3.12 & 0.45 \\
$\mathrm{CH}_{3} \mathrm{SH}$ & $1.9 \mathrm{E}-09$ & $1.3 \mathrm{E}-09$ & $8.1 \mathrm{E}-13$ & $2.9 \mathrm{E}-10$ & $3.7 \mathrm{E}-09$ & $1.6 \mathrm{E}-10$ & $2.5 \mathrm{E}-09$ & 3.18 & 2.29 & 0.45 \\
$\mathrm{CH}_{3} \mathrm{CHO}$ & $8.3 \mathrm{E}-10$ & $6.0 \mathrm{E}-10$ & $8.3 \mathrm{E}-14$ & $9.4 \mathrm{E}-11$ & $1.9 \mathrm{E}-10$ & $2.4 \mathrm{E}-11$ & $2.8 \mathrm{E}-10$ & 3.42 & 2.49 & 0.45 \\
$\mathrm{CH}_{3} \mathrm{NH} \mathrm{H}_{2}$ & $1.6 \mathrm{E}-09$ & $1.2 \mathrm{E}-09$ & $6.9 \mathrm{E}-13$ & $2.3 \mathrm{E}-10$ & $3.8 \mathrm{E}-09$ & $1.7 \mathrm{E}-10$ & $2.5 \mathrm{E}-09$ & 3.21 & 2.31 & 0.45 \\
$\mathrm{NH}_{2} \mathrm{CHO}$ & $5.2 \mathrm{E}-10$ & $4.0 \mathrm{E}-10$ & $2.8 \mathrm{E}-14$ & $4.9 \mathrm{E}-11$ & $3.0 \mathrm{E}-11$ & $2.0 \mathrm{E}-11$ & $1.5 \mathrm{E}-10$ & 3.57 & 2.62 & 0.45 \\
$\mathrm{C}_{2} \mathrm{H}_{5} \mathrm{OH}$ & $4.9 \mathrm{E}-10$ & $3.9 \mathrm{E}-10$ & $1.4 \mathrm{E}-14$ & $4.1 \mathrm{E}-11$ & - & $2.8 \mathrm{E}-11$ & $1.8 \mathrm{E}-10$ & 3.72 & 2.75 & 0.45 \\
$\mathrm{C}_{3} \mathrm{H}_{7} \mathrm{OH}$ & $7.7 \mathrm{E}-10$ & $6.0 \mathrm{E}-10$ & $2.7 \mathrm{E}-14$ & $6.6 \mathrm{E}-11$ & $4.4 \mathrm{E}-11$ & $4.3 \mathrm{E}-11$ & $2.9 \mathrm{E}-10$ & 3.71 & 2.74 & 0.45 \\
$\mathrm{SH}$ & $5.0 \mathrm{E}-11$ & $3.7 \mathrm{E}-11$ & $5.3 \mathrm{E}-16$ & $3.9 \mathrm{E}-12$ & - & $8.9 \mathrm{E}-13$ & $1.1 \mathrm{E}-11$ & 3.92 & 2.90 & 0.45 \\
$\mathrm{H}_{2} \mathrm{~S}$ & $7.8 \mathrm{E}-10$ & $5.9 \mathrm{E}-10$ & $4.6 \mathrm{E}-14$ & $7.8 \mathrm{E}-11$ & - & $2.6 \mathrm{E}-11$ & $2.0 \mathrm{E}-10$ & 3.53 & 2.58 & 0.45 \\
$\mathrm{CS}$ & $2.6 \mathrm{E}-11$ & $1.8 \mathrm{E}-11$ & $7.3 \mathrm{E}-16$ & $2.4 \mathrm{E}-12$ & - & $1.9 \mathrm{E}-13$ & $3.6 \mathrm{E}-12$ & 3.59 & 2.63 & 0.45 \\
$\mathrm{CS}$ & $3.6 \mathrm{E}-10$ & $2.6 \mathrm{E}-10$ & $2.0 \mathrm{E}-14$ & $3.4 \mathrm{E}-11$ & $1.6 \mathrm{E}-10$ & $2.1 \mathrm{E}-11$ & $2.0 \mathrm{E}-10$ & 3.57 & 2.62 & 0.45 \\
$\mathrm{OCS}_{2}$ & $7.7 \mathrm{E}-10$ & $6.1 \mathrm{E}-10$ & $1.1 \mathrm{E}-14$ & $6.1 \mathrm{E}-11$ & - & $3.0 \mathrm{E}-11$ & $2.2 \mathrm{E}-10$ & 3.85 & 2.86 & 0.45 \\
$\mathrm{~S}$ & $1.3 \mathrm{E}-10$ & $9.2 \mathrm{E}-11$ & $6.5 \mathrm{E}-14$ & $1.8 \mathrm{E}-11$ & $2.8 \mathrm{E}-10$ & $1.3 \mathrm{E}-11$ & $2.0 \mathrm{E}-10$ & 3.27 & 2.36 & 0.45 \\
$\mathrm{SO}$ & $5.3 \mathrm{E}-10$ & $3.7 \mathrm{E}-10$ & $3.2 \mathrm{E}-14$ & $5.7 \mathrm{E}-11$ & - & $6.1 \mathrm{E}-12$ & $9.8 \mathrm{E}-11$ & 3.46 & 2.52 & 0.45 \\
$\mathrm{SO}$ & $1.3 \mathrm{E}-10$ & $1.2 \mathrm{E}-10$ & $2.7 \mathrm{E}-16$ & $8.4 \mathrm{E}-12$ & - & $1.7 \mathrm{E}-11$ & $8.4 \mathrm{E}-11$ & 4.25 & 3.17 & 0.45 \\
$\mathrm{HCl}$ & $4.5 \mathrm{E}-11$ & $4.3 \mathrm{E}-11$ & - & $2.8 \mathrm{E}-12$ & - & $1.2 \mathrm{E}-11$ & $5.1 \mathrm{E}-11$ & 4.27 & 3.18 & 0.45 \\
$\mathrm{AlH}$ & $1.5 \mathrm{E}-10$ & $9.9 \mathrm{E}-11$ & $1.2 \mathrm{E}-12$ & $5.6 \mathrm{E}-11$ & $3.4 \mathrm{E}-10$ & $1.4 \mathrm{E}-11$ & $2.3 \mathrm{E}-10$ & 2.67 & 1.86 & 0.45 \\
\hline
\end{tabular}

short-wavelength limit to the radiation field in most cases. Conversely, the PHIDRATES cross sections are intended for studying photochemistry in the solar system, including highdensity environments like planetary atmospheres and cometary comae. Then, partial cross sections for many more dissociation and dissociative-ionisation fragments are considered, including metastable species, and with a stronger emphasis on the solar radiation field, favouring lower-lying states.

\section{Shielding functions}

\subsection{General formulation}

Substantial molecular abundances cannot exist in the unshielded interstellar medium because of their short dissociation lifetimes. Instead, observed molecules are found embedded inside interstellar clouds, protoplanetary disks, or similar objects that are at least partially shielded from radiation. The unattenuated photodissociation and photoionisation rates given in Sect. 5 must then be recalculated taking into account the intervening material, according to, for example,

$$
\begin{aligned}
k= & \int \sigma^{\mathrm{pd}}(\lambda) \exp \left[-\tau_{\text {dust }}\left(\lambda, N_{\mathrm{H}+2 \mathrm{H}_{2}}\right)\right. \\
& \left.-\sum_{X=\mathrm{H}, \mathrm{H}_{2}, \text { self }} N_{X} \sigma_{X}^{\mathrm{abs}}(\lambda)\right] I(\lambda) \mathrm{d} \lambda .
\end{aligned}
$$

The first exponentiated term in Eq. (9) models the attenuation of ultraviolet radiation due to dust as a function of wavelength and the column density of hydrogen nuclei (assuming this is proportional to the dust column). The non-absorbing scattering of UV photons by dust significantly alters their radiative transfer in a shielded region so that $\tau_{\text {dust }}\left(\lambda, N_{\mathrm{H}+2 \mathrm{H}_{2}}\right)$ is not simply proportional to column density. Additionally, the form of this term is dependent on the dust-cloud shape and nature of the incident radiation: isotropic, normal, or otherwise.

The summation term in Eq. (9) considers shielding by atomic and molecular species, where the most important cases are photoabsorption by $\mathrm{H}$ and $\mathrm{H}_{2}$, and self shielding for a few abundant species. The column density of species $X$ is represented by $N_{X}$.

The rate reduction due to dust, molecules, and atoms is characterised by a shielding function:

$\theta=\frac{k}{k_{0}}$

where, $k_{0}$ is the photoprocess rate at the irradiated edge of the shielded region. For an infinite-slab interstellar cloud in a region of space conforming to a standard isotropic ISRF, $k_{0}$ will be slightly greater than half of the rates in Tables 18 and 19, due to the restriction of incident radiation to $2 \pi$ sr and the occurrence of back-scattered radiation from the shielded region.

We calculated shielding functions according to Eqs. (9) and (10) for the photodissociation and ionisation of all atomic and molecular species in our database. These results are discussed below and are also available from the Leiden database ${ }^{15}$ in tabulated form. These tables contain values of $\theta$ as a function of column density for each bracketed term in Eq. (9) treated independently.

A demonstration of the relative importance of shielding terms in Eq. (9) is given by Fig. 25, which simulates the transmittance of ultraviolet radiation to a depth of $A_{\mathrm{V}}=1$ into an interstellar cloud. The column densities of intervening atomic and molecular species are the result of a particular diffuse-cloud chemical model run by Heays et al. (2014b), but their magnitudes are typical in an interstellar PDR. For wavelengths shorter than about $130 \mathrm{~nm}$ multiple sources contribute to ultraviolet shielding in this model. The combined shielding in this case is approximately a product of these, e.g., $\theta^{\text {total }}=\theta^{\text {dust }} \cdot \theta^{\mathrm{H}_{2}} \cdot \theta^{\mathrm{H}} \ldots$ This factorisation is somewhat inaccurate for multiple shielding species with overlapping and line-dominated cross sections. Additionally, the shielding functions calculated here are appropriate for infinite-slab geometries only. For more sophisticated astrochemical models the explicit simulation of ultraviolet radiative transfer using our database of cross sections may be required.

\footnotetext{
15 www.strw. leidenuniv.nl/ ewine/photo
} 

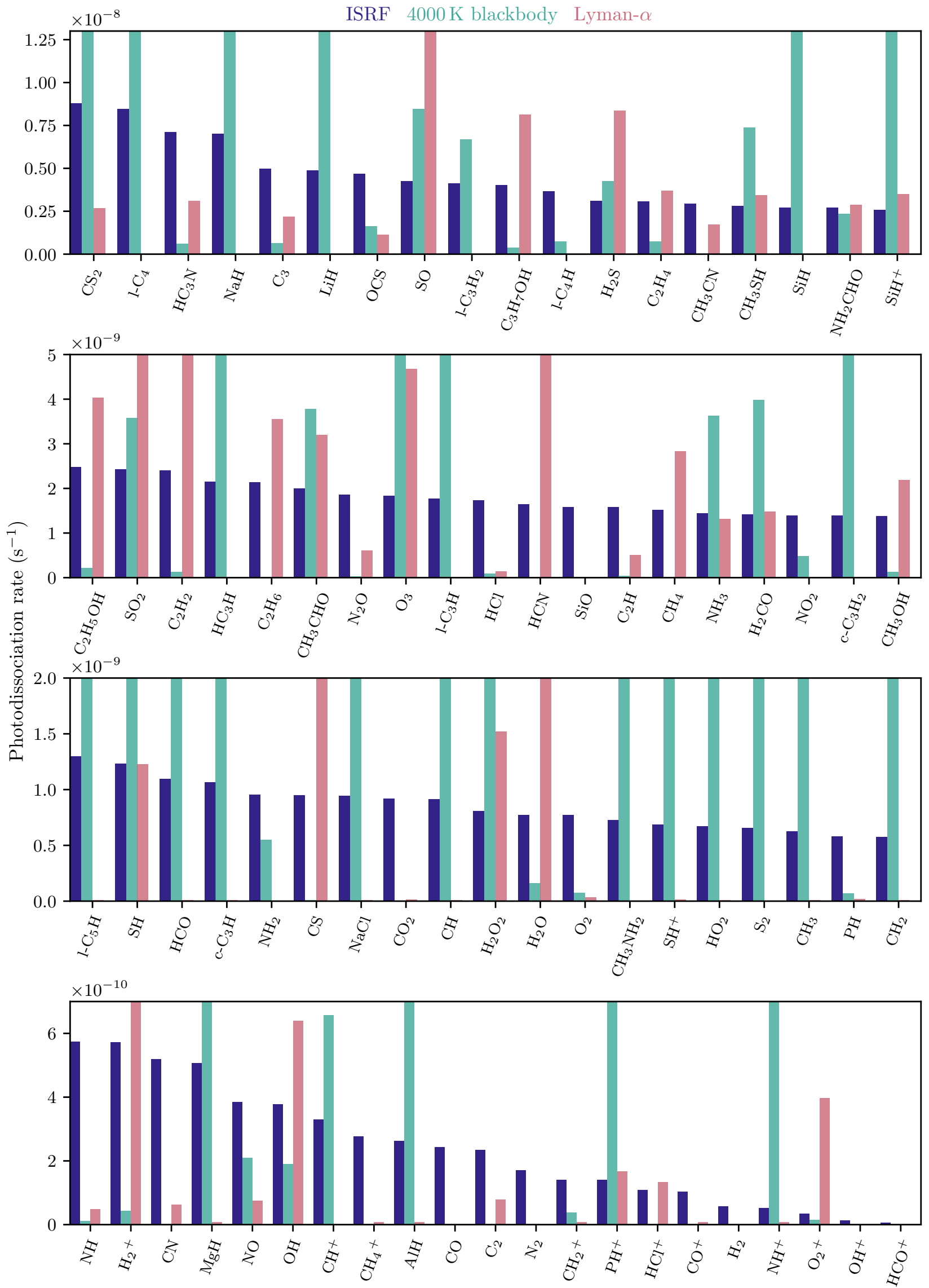

Fig. 19. Unshielded photodissociation rates of molecules. Rates are shown assuming three different radiation fields with ultraviolet intensity matching the standard of Draine (1978). 


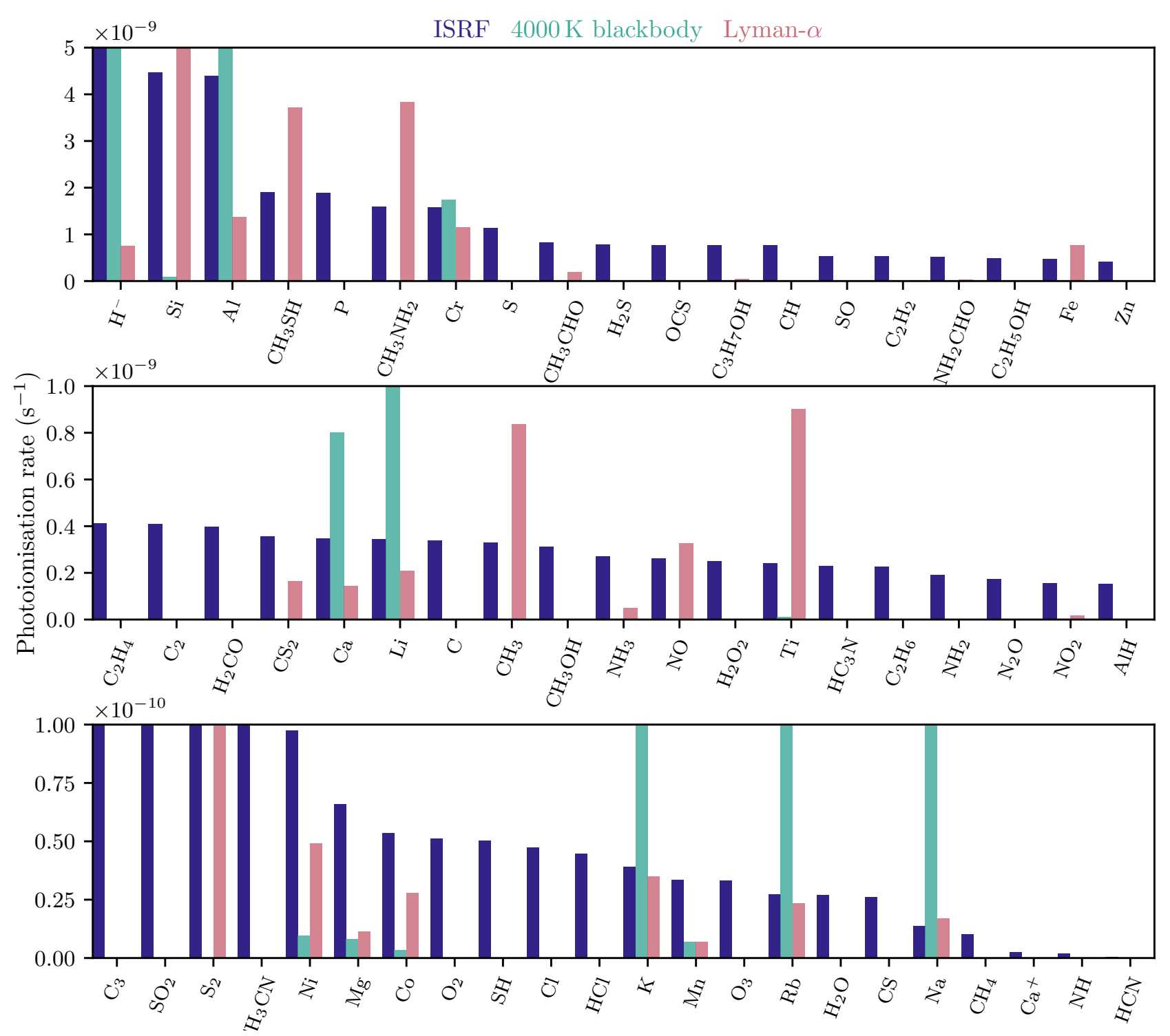

Fig. 20. Unshielded photoionisation rates of molecules and atoms. Rates are shown assuming different radiation fields with ultraviolet intensity matching the standard of Draine (1978).

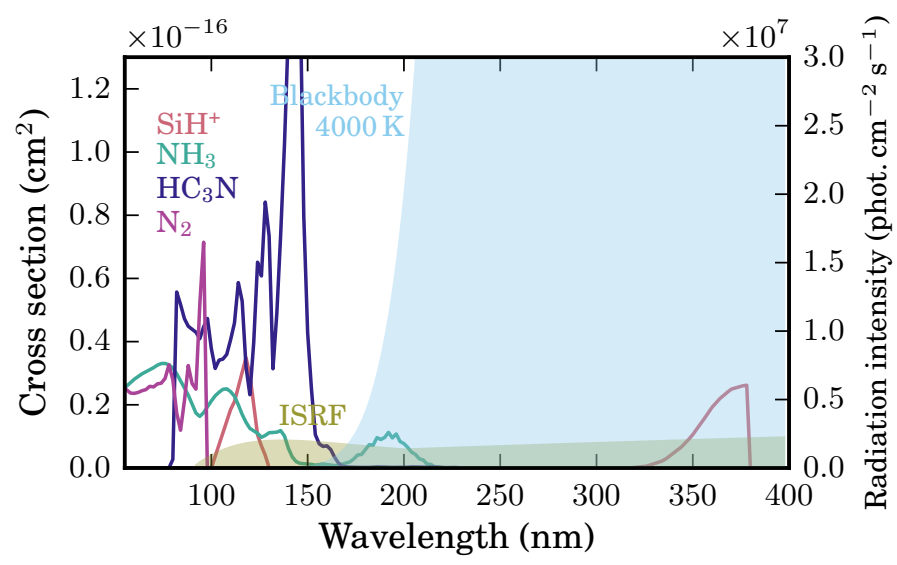

Fig. 21. Photodissociation cross sections of four molecules. These are averaged into $2 \mathrm{~nm}$ intervals for clarity and compared with the wavelength-dependence of two radiation fields from Sect. 2.

\subsection{Shielding by dust}

We calculated dust-shielding-dependent photodissociation and ionisation rates using the optical properties of a dust population assuming one particular composition and size distribution. These were taken from the mixed grain-size and composition model developed by Draine et al. (Draine \& Lee 1984; Li \& Draine 2001; Weingartner \& Draine 2001; Draine 2003a,b,c). We used their " $R_{\mathrm{V}}=3.1 "$ model published online ${ }^{16}$, with important optical properties plotted in Fig. 26. We also adopt a (gas mass)/(dust mass) ratio of 124 in line with the Draine et al. dust grain model. The interstellar variation of these parameters and their possible effects on shielding functions is discussed in Sect. 8.1.

The normal observationally-relevant extinction cross section is larger than the absorption cross section shown in Fig. 26 by a factor of $1 /(1-\omega)$, where $\omega$ is the grain albedo, to account for photons scattered out of the line of sight. These photons are however still available for photodestruction and their radiative transfer through an interstellar cloud must be considered.

\footnotetext{
16 www . astro.princeton. edu/ draine/dust/dustmix.html
} 


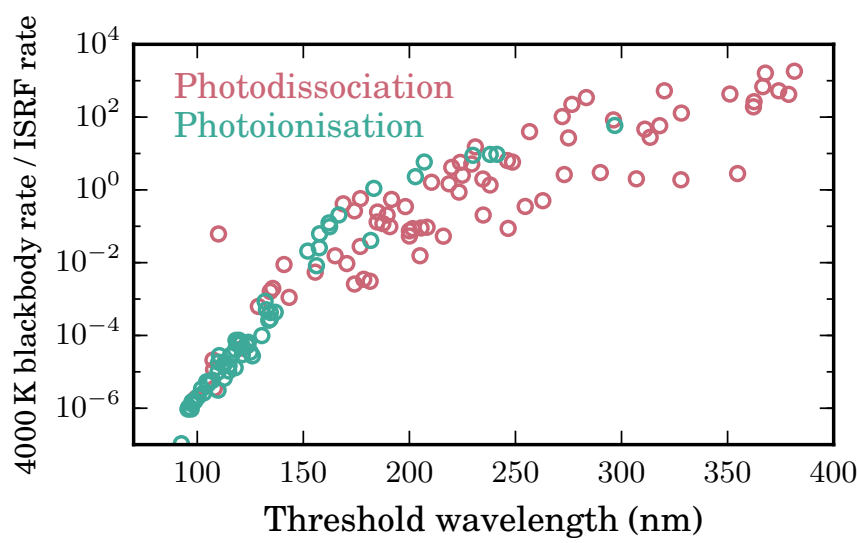

Fig. 22. Ratio of photodissociation and photoionisation rates. Calculated assuming two radiation fields from Sect. 2 as a function of their dissociation or ionisation thresholds.

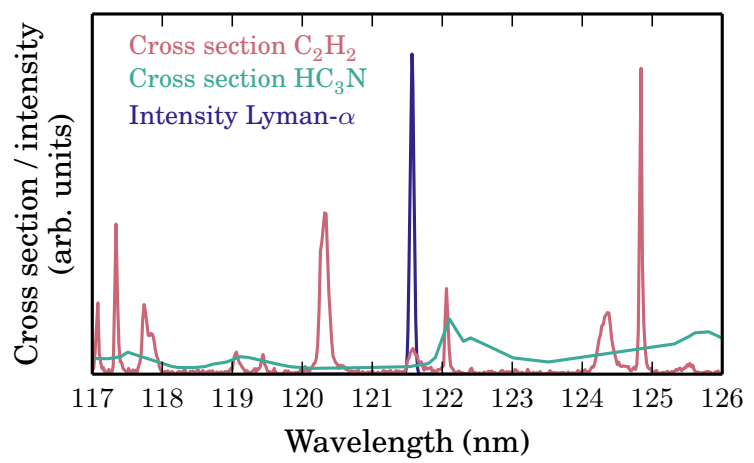

Fig. 23. Comparing the wavelength dependence of the photodissociation cross sections of $\mathrm{C}_{2} \mathrm{H}_{2}$ and $\mathrm{HC}_{3} \mathrm{~N}$ between 117 and $126 \mathrm{~nm}$ with Lyman- $\alpha$ radiation.

We did this for the case of an interstellar cloud with infiniteslab geometry and extending to large enough $A_{\mathrm{V}}$ that it is effectively illuminated from one side only, and assume this illumination is incident isotropically. The radiative transfer equations were solved according to the method of Roberge et al. (1991) and van Dishoeck et al. (2006) that takes into account the dust absorption cross section, dust albedo, and the averaged fraction of forward-scattered photons. All of these properties are significantly wavelength dependent in the ultraviolet spectral region, as shown in Fig. 26. The transmission through a dust thickness corresponding to $1 A_{\mathrm{V}}$, including scattered photons is shown in Fig. 25 and is largely wavelength independent longwards of about $120 \mathrm{~nm}$. At shorter-wavelength dust absorption rapidly becomes more effective.

The calculated wavelength-dependent penetration of ultraviolet photons was used to calculate depth-dependent photodestruction rates for each molecule and atom in our database. These are summarised as shielding functions in Fig. 27. The alternative $A_{\mathrm{V}}$ and H-nuclei column density scales are related by the standard proportionality (Savage et al. 1977)

$A_{\mathrm{V}}=N_{\mathrm{H}+2 \mathrm{H}_{2}} /\left(1.6 \times 10^{21} \mathrm{~cm}^{-2}\right)$

and assuming a gas-mass to dust-mass ratio of 124 . The differences between curves arise from the varying wavelength dependencies of atomic and molecular cross sections and the dust grain optical properties. All of the plotted shielding functions lie below the visual extinction curve, $\exp \left(-A_{\mathrm{V}}\right)$, despite the inclusion of forward scattering, which acts to increase the penetration depth. This is because of the larger dust absorption cross section at shorter wavelengths, which also explains the faster shielding of photoionisation than photodissociation, due to the shorterwavelength thresholds of ionisation.

Curves like those in Fig. 27 are sometimes approximated as simple functions of $A_{\mathrm{V}}$ for easier utilisation. Either, as oneparameter exponential curves,

$\theta\left(A_{\mathrm{V}}\right)=\exp \left(-\gamma_{\exp } A_{\mathrm{V}}\right)$

(e.g., van Dishoeck et al. 2006) or 2nd-order exponential integrals,

$\theta\left(A_{\mathrm{V}}\right)=\mathrm{E}_{2}\left(\gamma_{\mathrm{E}_{2}} A_{\mathrm{V}}\right)$

(e.g., Neufeld \& Wolfire 2009; Roueff et al. 2014), or biexponential functions (e.g. Roberge et al. 1991). We calculated values of $\gamma_{\exp }$ and $\gamma_{\mathrm{E}_{2}}$ in Eqs. (12) and (13) that best fit the results of our radiative transfer model, with an example fit for the shielded photodissociation of $\mathrm{CH}_{4}$ shown in Fig. 28. Calibrated exponential-integral functions were found to reproduce the shielding effects of dust absorption within $25 \%$ over the range of $1 \geq \theta>10^{-4}$, while the exponential function deviate by up to a factor of 3 over the same range. Given the superior parameterisation of exponential-integral functions, we listed $\gamma_{\mathrm{E}_{2}}$ coefficients for all species in our database in Tables 18 and 19, along with updated values of the normal-exponential $\gamma_{\text {exp }}$ parameters that are adopted in previous iterations of the Leiden database (van Dishoeck et al. 2006).

A further reduction of shielding functions is demonstrated in Fig. 28, whereby, an exponential-decay parameter $\gamma_{\mathrm{fit}}$ is adopted along with an effective unshielded rate, $k_{0}^{\text {fit }}$, so that the depthdependent photodestruction rates are:

$k=k_{0}^{\mathrm{fit}} \exp \left(\gamma_{\mathrm{fit}} A_{\mathrm{V}}\right)$

leading to shielding functions

$\theta=\frac{k_{0}^{\mathrm{fit}}}{k_{0}} \exp \left(\gamma_{\mathrm{fit}} A_{\mathrm{V}}\right)$

The values of these parameters were selected to best fit the radiative-transfer calculation between $A_{\mathrm{V}}=0.1$ and 3 (a range of extinction where the details of ISRF photodestruction has most influence on PDR chemistry), and provide a better approximation of this range than Eq. (12), but a poorer fit in general than Eq. (13). Fitted parameters of this modified exponential form are provided in the Leiden database for all atoms and molecules and may be useful for astrochemical codes where exponential-form dust shielding is required, but with improved accuracy over an intermediate range of $A_{\mathrm{V}}$.

The quantitative shielding discussion in this section is appropriate for use in single-sided isotropically-irradiated infiniteslab interstellar cloud models. The modelling of dust-shielding effects in other geometries would require specific radiativetransfer calculations.

Previously, van Dishoeck et al. (2006) compared dustshielding effects ad $\gamma_{\text {exp }}$ parameters for a range of radiation field types and dust grain properties, a point that is discussed further in Sect. 8.1. 

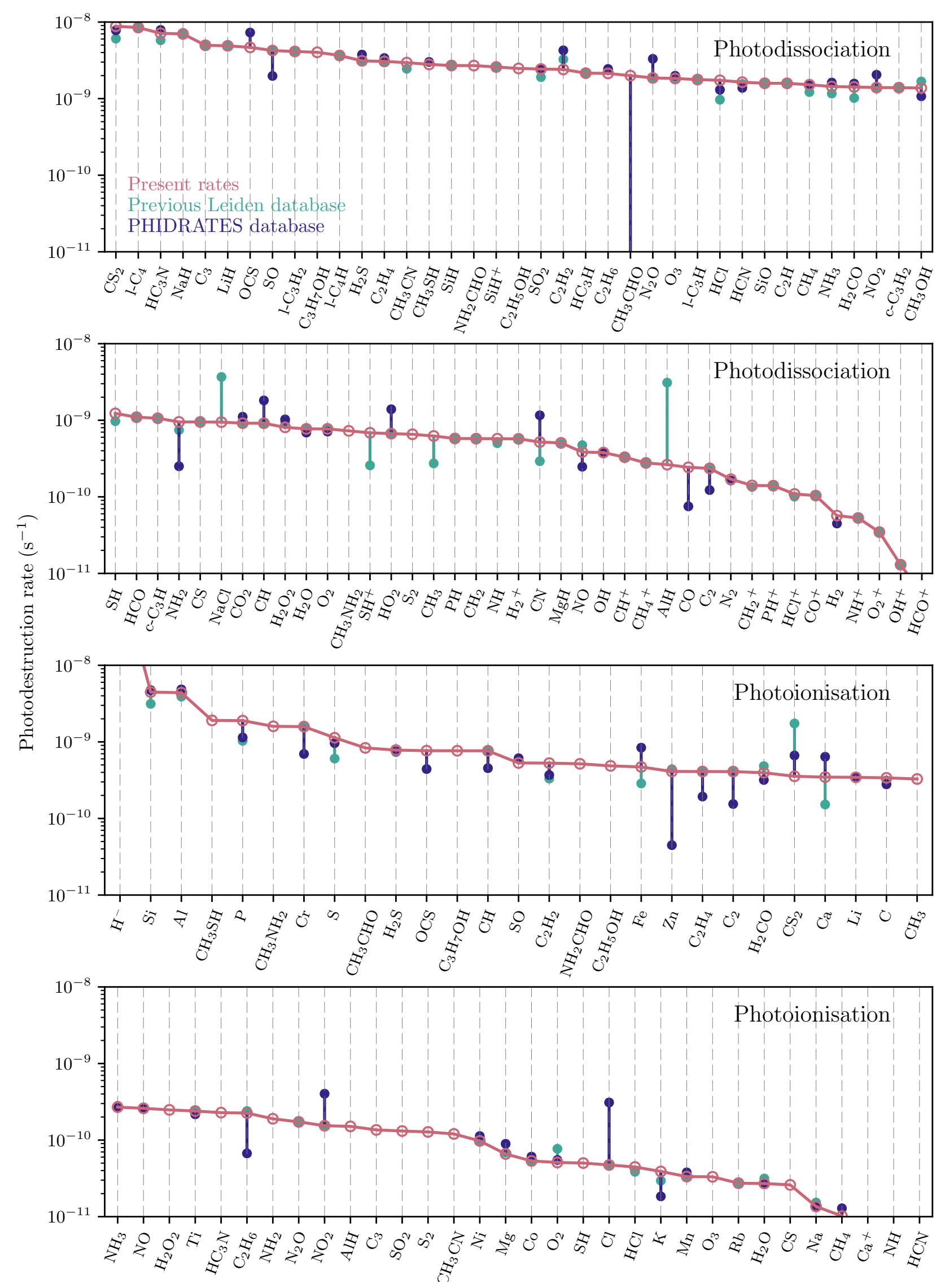

Fig. 24. ISRF photodissociation and ionisation rates for atoms and molecules. The present calculations are compared with the PHIDRATES database (Huebner et al. 1992; Huebner \& Mukherjee 2015) and the previous version of the Leiden database (van Dishoeck 1988; van Dishoeck et al. 2006). 
A. N. Heays et al.: Photodissociation and photoionisation of atoms and molecules of astrophysical interest

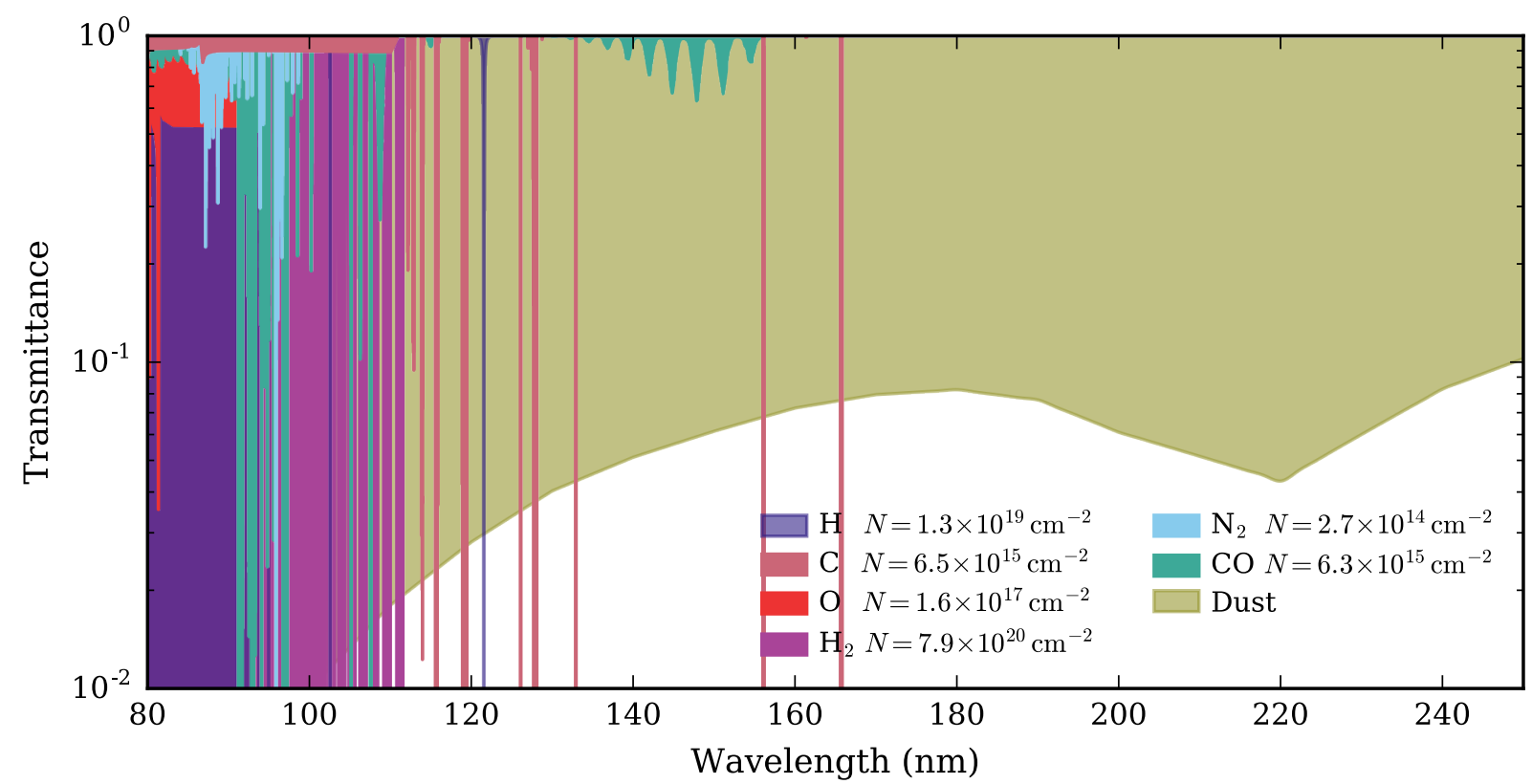

Fig. 25. Possible wavelength-dependent transmittance (shaded region edges) of the highest-column density $(N)$ species at $A_{V}=1$ depth into a semi-infinite one-sided interstellar cloud.

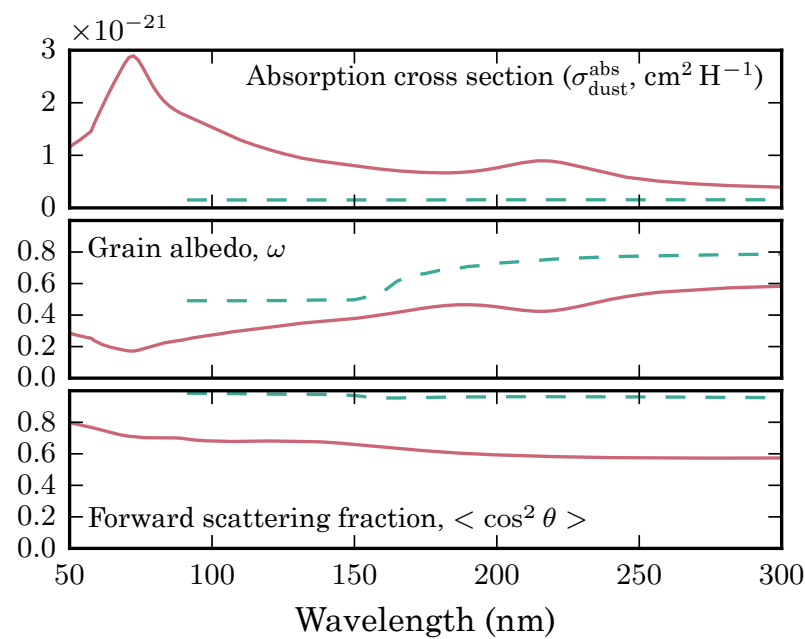

Fig. 26. Solid lines: optical properties of interstellar dust grains according to Draine et al. (Draine \& Lee 1984; Draine 2003b) ${ }^{17}$. Dashed lines: optical properties simulating grain growth in a protoplanetary disk. $U p$ per: photoabsorption and photoextinction cross sections. Middle: grain albedo. Lower: probability of forward scattering.

\subsection{Shielding by $\mathrm{H}_{2}, \mathrm{H}$, and $\mathrm{C}$}

The shielding of most molecules found in PDRs is dominated by dust extinction, assuming a standard amount of gas and dust. In some cases, additional terms in Eq. (9) must be considered.

The shielding effect on our database of molecules was calculated for a one-dimensional column of $\mathrm{H}_{2}$ while neglecting scattering in the molecular lines. Shielding functions calculated according by means of Eqs. (9) and (10) are summarised in Fig. 29 for two cases: the ISRF and TW-Hydra radiation fields. For these calculations, the $\mathrm{H}_{2}$ photoabsorption cross section was composed from a list of individual line parameters, as described

\footnotetext{
${ }^{17}$ Model $R_{\mathrm{V}}=3.1^{\prime}$ from www.astro.princeton.edu/ draine/
} dust/dustmix.html

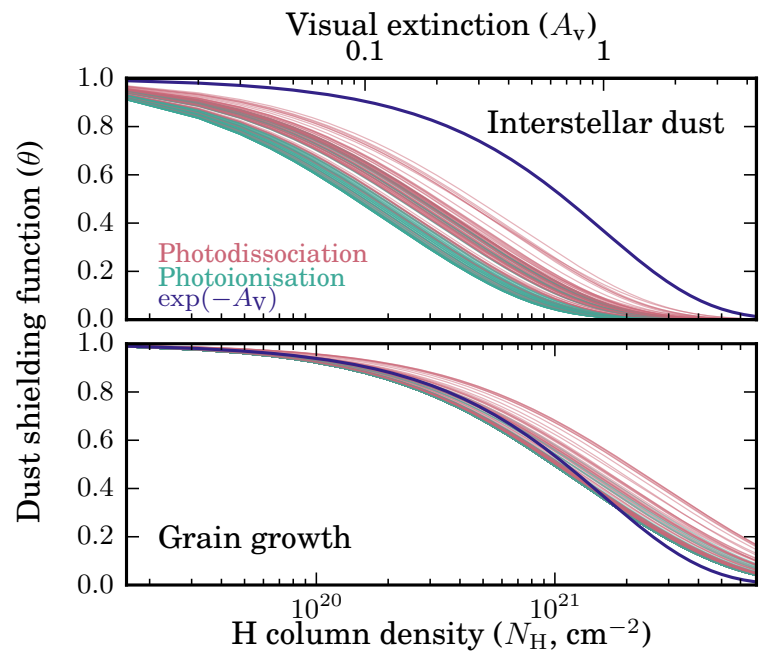

Fig. 27. Shielding of photodissociation (red) and photoionisation (green) in the ISRF. For all molecules in our database by an amount of dust parameterised by H-nucleus column density. Cases are shown for interstellar dust and a population of larger dust grains (see text). An additional curve traces $\exp \left(-A_{\mathrm{v}}\right)$, the visual extinction (blue).

in Sect. 4.3.15, and assumed an excitation temperature of $100 \mathrm{~K}$ and Gaussian Doppler broadening width $b=3 \mathrm{~km} \mathrm{~s}^{-1}$.

Significant $\mathrm{H}_{2}$ shielding only occurs in the ISRF for column densities of more than about $10^{20} \mathrm{~cm}^{-2}$. Larger columns are required for some molecules that photoabsorb at wavelengths greater than $110 \mathrm{~nm}$, outside the range of $\mathrm{H}_{2}$ line absorption. The shaded region in Fig. 29 shows a typical ultraviolet dustshielding curve for comparison. The influence of $\mathrm{H}_{2}$-shielding in the ISRF is overshadowed by dust extinction in many cases, assuming a standard gas and dust mass ratio, and can safely be neglected. In a few cases it is of comparable importance. For instance, $\mathrm{CO}$ and $\mathrm{N}_{2}$, investigated in detail by Visser et al. (2009) and $\mathrm{Li}$ et al. (2013). The extreme case of $\mathrm{H}_{2}$ shielding itself is discussed in the following section. 


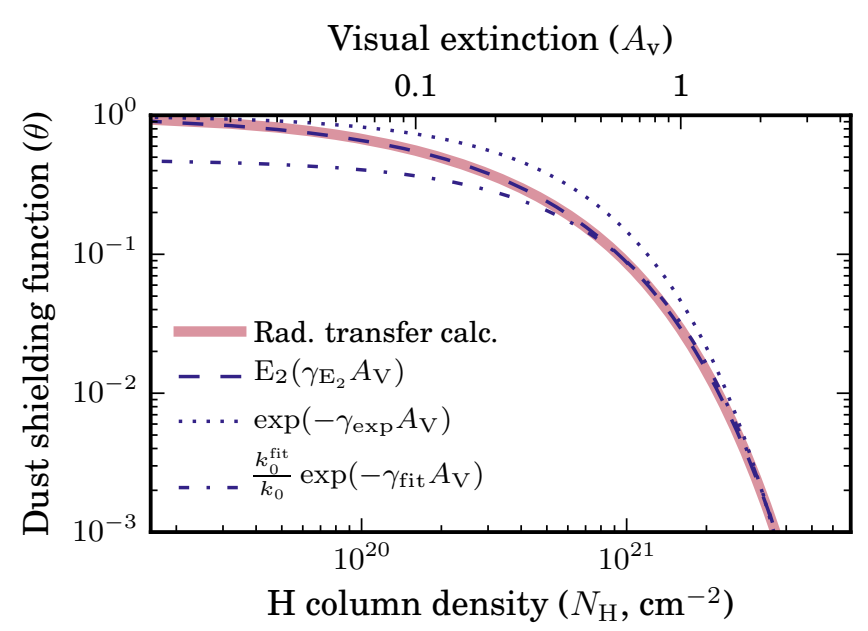

Fig. 28. Dust shielding function for $\mathrm{CH}_{4}$ photodissociation (solid red curve) in the ISRF. Calculated by means of an infinite-slab radiative transfer model. Also shown are some simple parameterisations of this curve according to three formulations.

\section{Visual extinction $\left(A_{\mathrm{v}}\right)$}

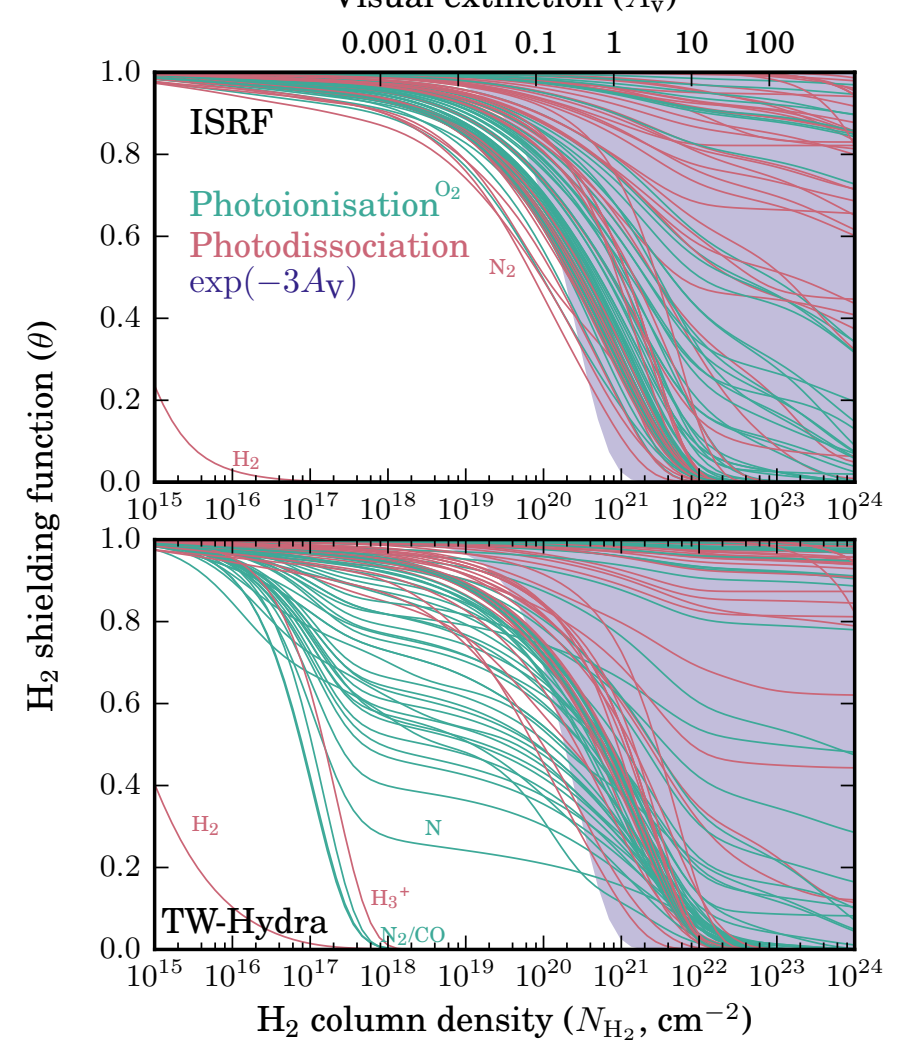

Fig. 29. Shielding functions for photodissociation (red curves) and photoionisation (blue curves) by a column of $\mathrm{H}_{2}$. Assumes an impinging ISRF or simulation of the TW-Hydra circumstellar radiation. A few extreme cases have their identity labelled. The shaded-region edge describes a typical ultraviolet-extinction curve due to interstellar dust, according to $\exp -3 A_{\mathrm{V}}$.

Figure 29 also shows $\mathrm{H}_{2}$ shielding functions assuming a radiation field simulating the TW-Hydra emission spectrum. The resulting changes with respect to the ISRF follow from the inclusion of radiation shorter than $91.2 \mathrm{~nm}$, so that the continuum absorption of $\mathrm{H}_{2}$ can rapidly reduce the photoionisation rate of some species. The shielding of longer-wavelength

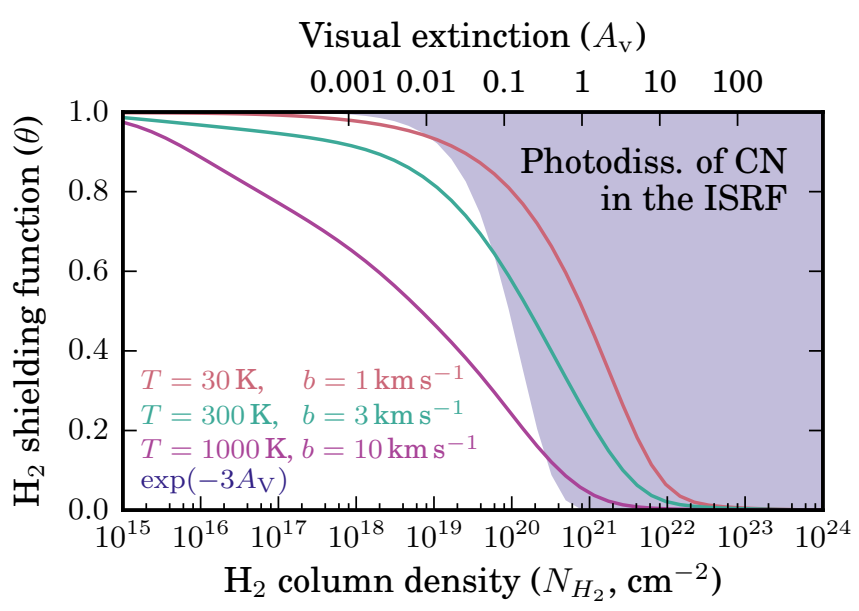

Fig. 30. Shielding of $\mathrm{CN}$ photodissociation in the ISRF by $\mathrm{H}_{2}$ assuming various values for the $\mathrm{H}_{2}$ rotational excitation temperature, $T$, and Doppler broadening, $b$. Also shown is a typical shielding function due to interstellar dust, $\exp \left(-3 A_{\mathrm{V}}\right)$ (shaded region edge).

photodissociation remains dust dominated apart from the case of $\mathrm{H}_{3}^{+}$, whose cross section occurs entirely at short wavelengths (Kulander \& Bottcher 1978).

An increase in the rotational temperature and turbulent broadening of $\mathrm{H}_{2}$ increases its shielding effectiveness, due to the larger filling factor of its photoabsorption spectrum when more and broader rotational lines are included. This effect is quite small, for molecules and atoms that primarily absorb longwards of $110 \mathrm{~nm}$. In some extreme cases the effect can be significant, as illustrated for $\mathrm{CN}$ photodissociation in Fig. 30. Here, increasing the temperature or Doppler width over astrophysically relevant ranges, from 30 to $300 \mathrm{~K}$ or 1 to $3 \mathrm{~km} \mathrm{~s}^{-1}$, respectively, leads to about twice the $\mathrm{H}_{2}$ shielding between 0.1 and $3 A_{\mathrm{V}}$. Increasing these further to $1000 \mathrm{~K}$ and $10 \mathrm{~km} \mathrm{~s}^{-1}$ (conditions perhaps still relevant to some interstellar shocks or in some atmospheres) results in an order of magnitude increase in $\mathrm{H}_{2}$ shielding effectiveness. The effect of $\mathrm{H}_{2}$ temperature and broadening are studied by Visser et al. (2009) and Li et al. (2013) for the cases of CO and $\mathrm{N}_{2}$, respectively.

Shielding by the atomic $\mathrm{H}$ photoionisation continuum is implicit in calculations with radiation fields having a $91.2 \mathrm{~nm}$ Lyman-limit cut-off. In principle, column-density-dependent $\mathrm{H}$-shielding occurs where UV radiation includes shorter wavelengths, although this detail is often negligible in view of the greater shielding effect of $\mathrm{H}_{2}$ and dust. Atomic-H shielding functions are discussed in Appendix A and may be important in dustdepleted environments near the edge of a PDR. Another species that potentially contributes to the exponential term in Eq. (9) is atomic C (Rollins \& Rawlings 2012), present near the boundary layer of a PDR, and we computed shielding functions for this species also, discussed further in Appendix A.

We did not evaluate the scattering of photons by $\mathrm{H}_{2}$ and $\mathrm{H}$, whereby photoabsorption into electronicallyexcited levels is followed by resonant photoemission (e.g., Black \& van Dishoeck 1987). This is shown to significantly modify the UV spectrum in embedded regions (Le Petit et al. 2006), as well as for Lyman- $\alpha$ photons scattered by atomic$\mathrm{H}$ (Neufeld 1991; Bethell \& Bergin 2011). For $\mathrm{H}_{2}$, more than $80 \%$ of absorbed UV photons are re-emitted, mostly to ground state levels with $v>0$ and at longer wavelengths. Ultimately, all photons will be absorbed by dust grains, but determining the 


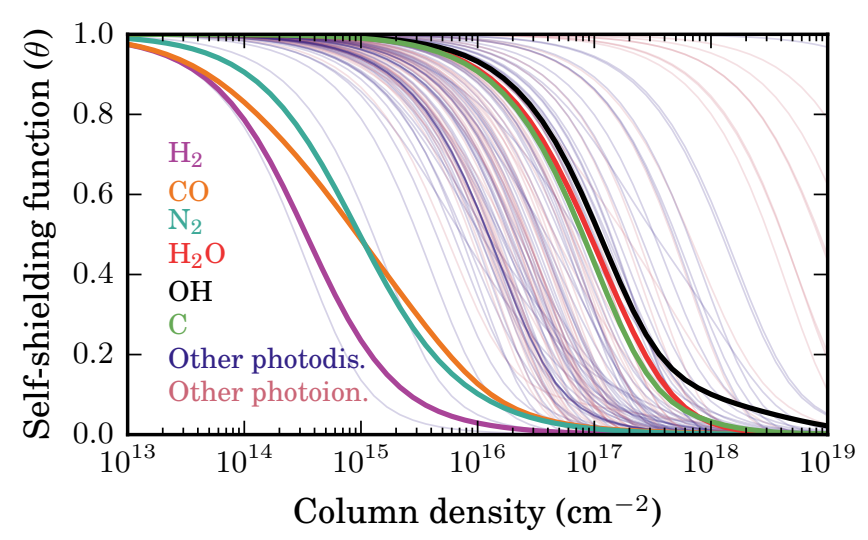

Fig. 31. Self-shielding of photodissociation in the ISRF.

influence of $\mathrm{H}_{2}$ resonant scattering on the photodestruction rates calculated here would be a worthwhile future project.

\subsection{Self-shielding of molecules}

Self-shielding is also important for some photodissociating species. Shielding functions describing this phenomenon independently of other mechanisms are plotted in Fig. 31. The three prominent cases where self-shielding is effective at relatively low column densities are $\mathrm{H}_{2}, \mathrm{CO}$, and $\mathrm{N}_{2}$. The most recent treatments of these three molecules are given in Sternberg et al. (2014), Visser et al. (2009), and Li et al. (2013), Heays et al. (2014b); respectively. The common cause is the line-like photoabsorption cross sections of all three molecules, leading to almost complete attenuation of ultraviolet radiation at their line centres for modest column densities, about $10^{15} \mathrm{~cm}^{-2}$. The column density of $\mathrm{CO}$ and $\mathrm{N}_{2}$ relative to $\mathrm{H}$ in the molecule-forming region of a PDR is typically high enough that their self-shielding competes with the simultaneous effect of dust extinction.

Atoms and molecules that absorb predominantly through continua may still be susceptible to self-shielding if their column densities are large enough. Three candidates for this phenomenon are highlighted in Fig. $31, \mathrm{H}_{2} \mathrm{O}, \mathrm{OH}$, and $\mathrm{C}$, where the minimum column density for effective self-shielding is approximately $10^{17} \mathrm{~cm}^{-2}$ in all cases. Such large columns of atomic $\mathrm{C}$ are actually found as discussed in the previous section, and some models of the inner regions of protoplanetary disks find sufficient $\mathrm{H}_{2} \mathrm{O}$ and $\mathrm{OH}$ columns (Bethell \& Bergin 2009; Ádámkovics et al. 2014). These phenomena may be particularly relevant in shocked media where densities are abnormally high, for example, $\mathrm{C}$ in supernova remnants and (White 1994) protoplanetary disks (Tsukagoshi et al. 2015), or $\mathrm{H}_{2} \mathrm{O}$ in protostellar outflows (Mottram et al. 2014). All other molecular species have too low abundance or insufficiently peaked cross sections to effectively self-shield in space.

\section{Photodestruction due to cosmic rays}

\subsection{Cosmic ray induced UV spectrum}

Cosmic rays penetrate deeper into an interstellar cloud, protostellar envelope, protoplanetary disk or planetary atmosphere than ultraviolet photons and ionise $\mathrm{H}_{2}$ there. This primary process and resultant cascade of re-scattered electrons proceeds to excite further $\mathrm{H}_{2}$ and generate excited $\mathrm{H}$ atoms (Cravens \& Dalgarno 1978; Gredel \& Dalgarno 1995),

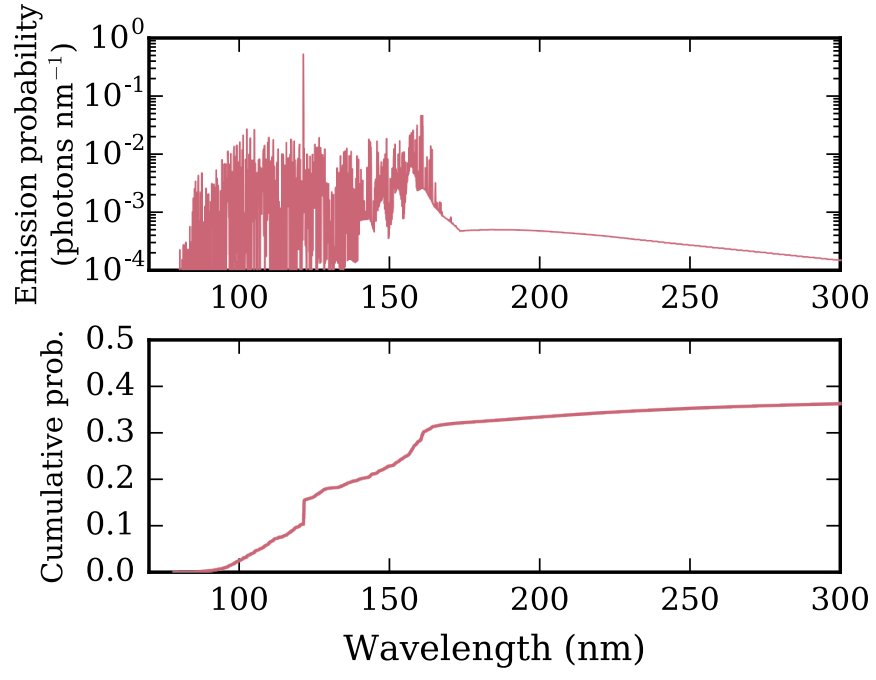

Fig. 32. The cosmic-ray induced radiation field. Upper: the spectral density of photons generated per primary cosmic-ray ionisation event. Lower: a cumulative integration of this distribution.

whose radiative decay generates a ultraviolet flux with a line-dominated structure (Prasad \& Tarafdar 1983; Gredel et al. 1987; Cecchi-Pestellini \& Aiello 1992). The photolysis of molecules due to this flux is quantified several times previously (Sternberg et al. 1987; Gredel et al. 1987, 1989; Heays et al. 2014 b) but the last major summary of rates dates back to Gredel et al. (1989). Here all rates are recomputed with updated cross sections. Also, the effects of grain growth, such as appropriate for protoplanetary disks, are considered.

The cosmic-ray induced ultraviolet flux is modelled here as a rate of photons generated per unit spectral density per hydrogen nucleus:

$R(\lambda)=\zeta_{\mathrm{H}_{2}} x_{\mathrm{H}_{2}} P(\lambda)$

Here, and below, $x_{\mathrm{X}}=n(\mathrm{X}) /\left[n(\mathrm{H})+2 n\left(\mathrm{H}_{2}\right)\right]$ is the relative abundance of species $\mathrm{X}$ with respect to total hydrogen nuclei and $\zeta_{\mathrm{H}_{2}}$ is the rate at which an $\mathrm{H}_{2}$ molecule is ionised by cosmic ray collisions. In the context of diffuse interstellar clouds (e.g., Oka 2013; Indriolo et al. 2015) it is atomic-H that is being ionised, and the appropriate $\zeta$ will then be per $\mathrm{H}$ atom.

We used the wavelength-dependent probability distribution of generated photons, $P(\lambda)$, of Gredel et al. (1989). This distribution is plotted in Fig. 32 and consists of many $\mathrm{H}_{2}$ and $\mathrm{H}$ emission lines between 80 and $170 \mathrm{~nm}$ as well as some continuum emission between 122 and $300 \mathrm{~nm}$. The modelled photoemission mostly occurs between 90 and $170 \mathrm{~nm}$, as shown by the rapid increase of the cumulative distribution in Fig. 32 over this range, with a single step at $121.6 \mathrm{~nm}$ constituting $15 \%$ of the integrated flux due to Lyman- $\alpha$ emission. The cumulative distribution attains a total value of only 0.36 because not every $\mathrm{H}_{2}$ ionisation event results in an ultraviolet photon being generated.

The value of $\zeta_{\mathrm{H}_{2}}$ is uncertain due to the unknown origin and flux of cosmic rays and its energy spectrum, as well as the attenuation due to matter and magnetic fields (Dalgarno 2006; Grenier et al. 2015). Earlier values are indirectly deduced from observations of the $\mathrm{HD}$ and $\mathrm{OH}$ abundances (e.g., Hartquist et al. 1978; van Dishoeck \& Black 1986) and $\mathrm{H}_{3}^{+}$abundances (e.g., van der Tak \& van Dishoeck 2000; Hezareh et al. 2008) in diffuse and dense interstellar clouds, and favoured a value of about $3 \times 10^{-17} \mathrm{~s}^{-1} \mathrm{H}_{2}-1$, although rates up to $2 \times 10^{-16} \mathrm{~s}^{-1}$ are inferred 
for some diffuse clouds. Subsequent observations towards more lines of sight and of other tracers like $\mathrm{OH}^{+}$and $\mathrm{H}_{2} \mathrm{O}^{+}$are interpreted with updated $\mathrm{H}_{3}^{+}$dissociative recombination rate coefficients generally and require a higher rate in diffuse clouds, 1 to $4 \times 10^{-15} \mathrm{~s}^{-1} \mathrm{H}_{2}^{-1}$ (Indriolo \& McCall 2012; Rimmer et al. 2012; Vaupré et al. 2014; Indriolo et al. 2015), and higher still in the Galactic centre. A reduction of the ionisation rate likely occurs in dark clouds and protoplanetary disks because of the shielding effects of the surrounding material (Padovani et al. 2009) or stellar winds and magnetic fields (Cleeves et al. 2013, 2015).

We adopt a primary ionisation rate for Eq. (16) of $\zeta_{\mathrm{H}_{2}}=$ $10^{-16} \mathrm{~s}^{-1} \mathrm{H}_{2}{ }^{-1}$. All molecular photodissociation rates can be adapted to an alternative $\zeta_{\mathrm{H}_{2}}$ by simple scaling.

Most cosmic-ray generated photons are eventually eliminated through absorption by dust grains inside an interstellar cloud but some excite atoms and molecules. The fraction of photons that lead to the photodissociation or ionisation of an atom or molecule, $\mathrm{X}$, is given by

$$
p_{\mathrm{X}}(\lambda)=\frac{x_{\mathrm{X}} \sigma_{\mathrm{X}}^{\mathrm{diss} / \mathrm{ion}}(\lambda)}{x_{\mathrm{dust}} \sigma_{\mathrm{dust}}^{\mathrm{abs}}(\lambda)+\sum_{j} x_{j} \sigma_{j}^{\mathrm{abs}}(\lambda)} .
$$

Here, $\sigma_{\mathrm{X}}^{\mathrm{diss} / i \mathrm{in}}$ is the photodissociation or ionisation cross section of species $\mathrm{X}$, and the denominator sums the photoabsorption cross section of all dust and gas species. The probability of a cosmic-ray generated ultraviolet photon being absorbed by a dust grain, $\mathrm{H}_{2}$ molecule, or some other gas-phase species depends on its wavelength through the various cross sections in Eq. (17).

The rate of a particular photodestruction process for species $\mathrm{X}$ (per $\mathrm{X}$ ) due to cosmic-ray-induced photons is then

$k_{\mathrm{X}}=\frac{1}{x_{\mathrm{X}}} \int R(\lambda) p_{\mathrm{X}}(\lambda) \mathrm{d} \lambda$.

The photolysis rates calculated by Gredel et al. (1989) and McElroy et al. (2013) are presented as efficiencies with the $\mathrm{H}_{2}$ ionisation rate and grain albedo factored out from Eq. (18). This is not possible if the summation terms in Eq. (17) are significant (Gredel et al. 1987; Heays et al. 2014b), and for the sake of generality we did not make this reduction. Our calculated rates divided by a factor of $2 \times 10^{16}$ are approximately comparable with the efficiencies given in Gredel et al. (1989), and a factor of $10^{16}$ is required when comparing with McElroy et al. (2013).

The spectrum shown in Fig. 32 and used in our rate calculations assumes an ortho- $\mathrm{H}_{2}$ :para- $\mathrm{H}_{2}$ ratio of $0: 1$, that is, with $\mathrm{H}_{2}$ in its $J=0$ rotational state. This is appropriate for excitation temperatures corresponding to the low temperatures of a molecular cloud because only $10 \%$ of equilibrated $\mathrm{H}_{2}$ is excited above $J=0$ at, for example, $50 \mathrm{~K}$. Even for cases where significant quantities of super-thermally excited $\mathrm{H}_{2}$ are inferred in interstellar clouds their influence on cosmic-ray induced ultraviolet photodissociation is unlikely to be large. This is because an altered distribution of emission lines is largely washed out in rate calculation by the integration in Eq. (18). Indeed, after testing $J=0: J=1$ populations with the two ratios 1:0 and $1: 3$ we find calculated-rate differences of less than $20 \%$ for most species in our database, in line with previous work (Gredel et al. 1989). Larger differences are found for the case of CO and $\mathrm{N}_{2}$, which are studied in more detail previously (Gredel et al. 1987; Heays et al. 2014b).

Gredel et al. (1989) considered the possibility of $\mathrm{H}_{2}$ bound levels absorbing the cosmic-ray induced ultraviolet flux and subsequently re-emitting photons of the same wavelength or longer (following emission into excited ground state vibrational levels). Their calculated rates are altered by up to $35 \%$ by considering this phenomenon, with most species being altered by less than $5 \%$, and this effects is neglected here.

For the case of dust absorption, the wavelength-dependent mixed-grain absorption cross section of Draine et al. (Draine \& Lee 1984; Li \& Draine 2001; Weingartner \& Draine 2001; Draine 2003b,c) were used, as discussed in Sect. 5 and plotted in Fig. 26. This absorption cross section is somewhat different from the wavelength-independent dust properties adopted previously (Gredel et al. 1987; Heays et al. 2014b) that converted an observationally-estimated dust extinction cross section, $2 \times 10^{-21} \mathrm{~cm}^{-1} \mathrm{H}^{-1}$, to an absorption cross section by assuming an effective grain albedo between 0 and 0.8 . It is clear from Fig. 26 that consideration of the wavelength dependence of dust absorption can affect the deduced shielding of molecular photodestruction by, at most, a factor of about two, depending on the wavelength-dependence of the molecular photoabsorption cross section.

\subsection{Results}

Photodissociation and ionisation rates in a cosmic-ray induced ultraviolet field were calculated for all molecules in our database after adopting the following set of parameters: Cosmic-ray ionisation rate $\left(\zeta_{\mathrm{H}_{2}}\right): 10^{-16} \mathrm{~s}^{-1} \mathrm{H}_{2}^{-1}$;

Doppler broadening $(b)$ : $\quad 1 \mathrm{~km} \mathrm{~s}^{-1}$; ortho- $\mathrm{H}_{2}$ :para- $\mathrm{H}_{2}$ : $x(\mathrm{H})$ :

$x_{\mathrm{N}_{2}}$ and $x_{\mathrm{CO}}$ :

$0: 1$;

$10^{-4}$

$10^{-5}$

where $x$ is the abundance relative to H-nuclei.

The integration in Eq. (18) was performed on a wavelength grid with $0.001 \mathrm{~nm}$ (or finer) resolution, in order to capture full details of the cross section structure of absorbing species. The calculated photodissociation and photoionisation rates are presented in Table 20. The ISRF-weighted uncertainties listed in Table 1 provide a reasonable uncertainty estimate for these rates.

A comparison of rates for molecules common to our database and that of Gredel et al. (1989) is plotted in Fig. 33. Also shown are rates calculated while neglecting the shielding of radiation by $\mathrm{H}_{2}$ and $\mathrm{H}$, and assuming a constant dust absorption cross section, $10^{-21} \mathrm{~cm}^{2} \mathrm{H}^{-1}$. These changes alter the rates in our database by factors between 0.5 and 5 , the increase occurring for species absorbing mostly shortwards of $110 \mathrm{~nm}$ where the $\mathrm{H}_{2}$ photoabsorption is greatest and the wavelength-dependent dust absorption cross section is also greater than its average. These "simple dust" rates in Fig. 33 are in line with the assumption adopted by Gredel et al. (1989), assuming a dust grain albedo of 0.5. Then, any further differences between rates is due to the change in our photodissociation cross sections relative to the previous work.

Overall, agreement is within a factor of two, with some exceptions. The largest rate we find is for the dissociation of $\mathrm{C}_{3}$, with a 5-fold increase relative to Gredel et al. (1989), due to the addition of a previously-unknown and strong $\mathrm{C}_{3}$ absorption channel at $160 \mathrm{~nm}$ as computed by van Hemert \& van Dishoeck (2008) and seen in the laboratory by Monninger et al. (2002). The new $\mathrm{CH}_{3} \mathrm{OH}$ dissociation and ionisations rates are 2.5 times smaller than that calculated by Gredel et al. (1989), who employed very similar cross sectional data (Harrison et al. 1959; Salahub \& Sandorfy 1971) over the range 120 to $215 \mathrm{~nm}$. Additional information described in Sect. 4.3.52 has permitted us to extend this cross section shortwards to $66 \mathrm{~nm}$, without which 
Table 20. Cosmic ray photodestruction rates $\left(\times 10^{-16} \mathrm{~s}^{-1}\right)$.

\begin{tabular}{|c|c|c|c|c|c|c|c|c|}
\hline Species & Diss. $^{a}$ & Ion. $^{a}$ & Species & Diss. & Ion. & Species & Diss. & Ion. \\
\hline $\mathrm{H}$ & - & - & $\mathrm{C}_{2}$ & 180 & 250 & $\mathrm{NO}_{2}$ & 1000 & 110 \\
\hline $\mathrm{Li}$ & - & 250 & $\mathrm{C}_{2} \mathrm{H}$ & 1100 & - & $\mathrm{N}_{2} \mathrm{O}$ & 1400 & 99 \\
\hline $\mathrm{C}$ & - & 260 & $\mathrm{C}_{2} \mathrm{H}_{2}$ & 3500 & 380 & $\mathrm{CN}$ & 450 & 8.3 \\
\hline $\mathrm{N}$ & - & - & $\mathrm{C}_{2}^{2} \mathrm{H}_{4}^{2}$ & 3500 & 280 & $\mathrm{HCN}$ & 2000 & 14 \\
\hline $\mathrm{O}$ & - & 2.7 & $\mathrm{C}_{2} \mathrm{H}_{6}$ & 2100 & 180 & $\mathrm{HC}_{3} \mathrm{~N}$ & 5900 & 160 \\
\hline $\mathrm{Na}$ & - & 13 & $\mathrm{C}_{3}$ & 6900 & 89 & $\mathrm{CH}_{3} \mathrm{OH}$ & 1600 & 240 \\
\hline $\mathrm{Mg}$ & - & 110 & $l-\mathrm{C} 3 \mathrm{H}$ & 3000 & - & $\mathrm{CH}_{3}^{3} \mathrm{CN}$ & 2600 & 97 \\
\hline $\mathrm{Al}$ & - & 2500 & $c-\mathrm{C} 3 \mathrm{H}$ & 480 & - & $\mathrm{CH}_{3} \mathrm{SH}$ & 2700 & 2000 \\
\hline $\mathrm{Si}$ & - & 4200 & $\mathrm{HC}_{3} \mathrm{H}$ & 1100 & - & $\mathrm{CH}_{3} \mathrm{CHO}$ & 2200 & 690 \\
\hline $\mathrm{P}$ & - & 1500 & $l-\mathrm{C}_{3} \mathrm{H}_{2}$ & 3400 & - & $\mathrm{CH}_{3} \mathrm{NH}_{2}$ & 450 & 1800 \\
\hline$S$ & - & 800 & $c-\mathrm{C}_{3} \mathrm{H}_{2}$ & 690 & - & $\mathrm{NH}_{2} \mathrm{CHO}$ & 2900 & 410 \\
\hline $\mathrm{Cl}$ & - & 47 & $l-\mathrm{C}_{4}$ & 1800 & - & $\mathrm{C}_{2} \mathrm{H}_{5} \mathrm{OH}$ & 2600 & 380 \\
\hline $\mathrm{K}$ & - & 34 & $l-\mathrm{C}_{4} \mathrm{H}$ & 6100 & - & $\mathrm{C}_{3} \mathrm{H}_{7} \mathrm{OH}$ & 4600 & 590 \\
\hline $\mathrm{Ca}$ & - & 270 & $l-\mathrm{C}_{5}^{4} \mathrm{H}$ & 170 & - & $\mathrm{SH}$ & 1100 & 34 \\
\hline $\mathrm{Ti}$ & - & 230 & $\mathrm{OH}$ & 470 & - & $\mathrm{SH}^{+}$ & 460 & - \\
\hline $\mathrm{Cr}$ & - & 1200 & $\mathrm{OH}^{+}$ & 8.6 & - & $\mathrm{H}_{2} \mathrm{~S}$ & 3400 & 620 \\
\hline Mn & - & 49 & $\mathrm{H}_{2} \mathrm{O}$ & 1000 & 23 & $\mathrm{CS}$ & 1900 & 20 \\
\hline $\mathrm{Fe}$ & - & 480 & $\mathrm{O}_{2}$ & 780 & 28 & $\mathrm{CS}_{2}$ & 5500 & 310 \\
\hline $\mathrm{Co}$ & - & 60 & $\mathrm{O}_{2}^{+}$ & 70 & - & OCS & 5200 & 560 \\
\hline $\mathrm{Ni}$ & - & 140 & $\mathrm{HO}_{2}$ & 190 & - & $\mathrm{S}_{2}$ & 88 & 140 \\
\hline $\mathrm{Zn}$ & - & 180 & $\mathrm{H}_{2} \mathrm{O}_{2}$ & 830 & 180 & SO & 5500 & 450 \\
\hline $\mathrm{Rb}$ & - & 23 & $\mathrm{O}_{3}$ & 1500 & 32 & $\mathrm{SO}_{2}$ & 2700 & 110 \\
\hline $\mathrm{Ca}^{+}$ & - & 1.5 & $\mathrm{CO}$ & $46^{b}$ & 14 & $\mathrm{SiH}$ & 620 & - \\
\hline $\mathrm{H}^{-}$ & - & 1300 & $\mathrm{CO}^{+}$ & 77 & - & $\mathrm{SiH}^{+}$ & 1200 & - \\
\hline $\mathrm{H}_{2}$ & - & - & $\mathrm{CO}_{2}$ & 600 & 8.2 & $\mathrm{SiO}$ & 890 & - \\
\hline $\mathrm{H}_{2}^{+}$ & 610 & - & $\mathrm{HCO}$ & 530 & - & $\mathrm{HCl}$ & 1500 & 46 \\
\hline $\mathrm{H}_{3}^{+}$ & - & - & $\mathrm{HCO}^{+}$ & 3.3 & - & $\mathrm{HCl}^{+}$ & 97 & - \\
\hline $\mathrm{CH}$ & 1100 & 580 & $\mathrm{H}_{2} \mathrm{CO}$ & 1300 & 290 & $\mathrm{NaCl}$ & 180 & - \\
\hline $\mathrm{CH}^{+}$ & 220 & - & $\mathrm{NH}$ & 370 & 7.1 & $\mathrm{PH}$ & 720 & - \\
\hline $\mathrm{CH}_{2}$ & 290 & - & $\mathrm{NH}^{+}$ & 22 & - & $\mathrm{PH}^{+}$ & 90 & - \\
\hline $\mathrm{CH}_{2}^{+}$ & 89 & - & $\mathrm{NH}_{2}$ & 720 & 140 & $\mathrm{AlH}$ & 54 & 150 \\
\hline $\mathrm{CH}_{3}$ & 280 & 380 & $\mathrm{NH}_{3}$ & 1100 & 220 & $\mathrm{LiH}$ & 620 & - \\
\hline $\mathrm{CH}_{4}^{3}$ & 1500 & 22 & $\mathrm{~N}_{2}{ }^{3}$ & $39^{c}$ & - & $\mathrm{MgH}$ & 250 & - \\
\hline $\mathrm{CH}_{4}^{+}$ & 270 & - & $\mathrm{NO}$ & 300 & 240 & $\mathrm{NaH}$ & 930 & - \\
\hline
\end{tabular}

Notes. ${ }^{(a)}$ Assumes a cosmic ray ionisation rate of $\zeta_{\mathrm{H}_{2}}=10^{-16} \mathrm{~s}^{-1} \mathrm{H}_{2}{ }^{-1}$. A simple scaling recovers photodestruction rates for other values of $\zeta_{\mathrm{H}_{2}} \cdot{ }^{(b)}$ Assumes an abundance relative to H-nuclei of $x_{\mathrm{CO}}=10^{-5}$ and significant self-shielding. Rate neglecting self-shielding: $9.9 \times 10^{-15} \mathrm{~s}^{-1}$. (c) Assumes an abundance relative to H-nuclei of $x_{\mathrm{N}_{2}}=10^{-5}$ and significant self-shielding. Rate neglecting self-shielding: $1.2 \times 10^{-14} \mathrm{~s}^{-1}$.

our cosmic-ray photodestruction rates would actually be substantially smaller. The difference between our calculation and Gredel et al. is therefore unresolved. The new $\mathrm{HC}_{3} \mathrm{~N}$ rate is 3 times larger than that calculated by Gredel et al. employing the cross section of Connors et al. (1974), which is nonetheless very similar to the data collected here, which we also are unable to immediately explain.

The three-times-increased $\mathrm{HCl}$ rate is due to the addition of higher-lying photodissociating transitions (described in Sect. 4.3.70) than included in the calculation of van Dishoeck et al. (1982), adopted previously. A similar explanation leads to the large change in the estimated $\mathrm{NH}_{2}$ dissociation and ionisation rates. Finally, the $\mathrm{CN}$ photodissociation rate was reduced by more than 10 times relative to Gredel et al., who adopted a earlier theoretical cross section (Lavendy et al. 1984) for this molecule that has since been updated (see Sect. 4.3.49 and Lavendy et al. 1987).

Similar to the conclusions of Sect. 6, self-shielding of the cosmic-ray flux was only found to be important for species with highly-structured cross sections and high abundance, that is,
$\mathrm{N}_{2}$ and CO (Heays et al. 2014b; Gredel et al. 1987). The photodissociation rates of these species are reduced through selfshielding by about $50 \%$ after assuming abundances relative to $\mathrm{H}$-nuclei of $10^{-5}$, as is typical for diffuse and dense interstellar clouds and prestellar cores (Tielens 2013), as long as the dust temperature is sufficiently high to prevent condensation of $\mathrm{CO}$ and $\mathrm{N}_{2}$ onto dust grains, that is greater than about 25 and $20 \mathrm{~K}$, respectively.

A relative abundance of about $10^{-7}$ is predicted for $\mathrm{H}_{2} \mathrm{O}, \mathrm{OH}$, $\mathrm{CO}_{2}, \mathrm{NH}_{3}$, and $\mathrm{CH}_{4}$ in some specific models of prestellar cores and dense clouds, for example, Tielens (2013), with the abundance all other species being $10^{-8}$ or below. No species in our database achieves a self-shielding effect of more than $4 \%$ at the $10^{-7}$ abundance level.

The assumed $\mathrm{H}, \mathrm{CO}$, and $\mathrm{N}_{2}$ abundances contribute to line-shielding of the cosmic ray induced UV flux and subsequent reduction of the photoabsorption rates of other species. These abundances are, however, dependent on the cosmic-ray ionisation rate itself through the induced chemistry and other dynamical factors like temperature and evolutionary age. The 

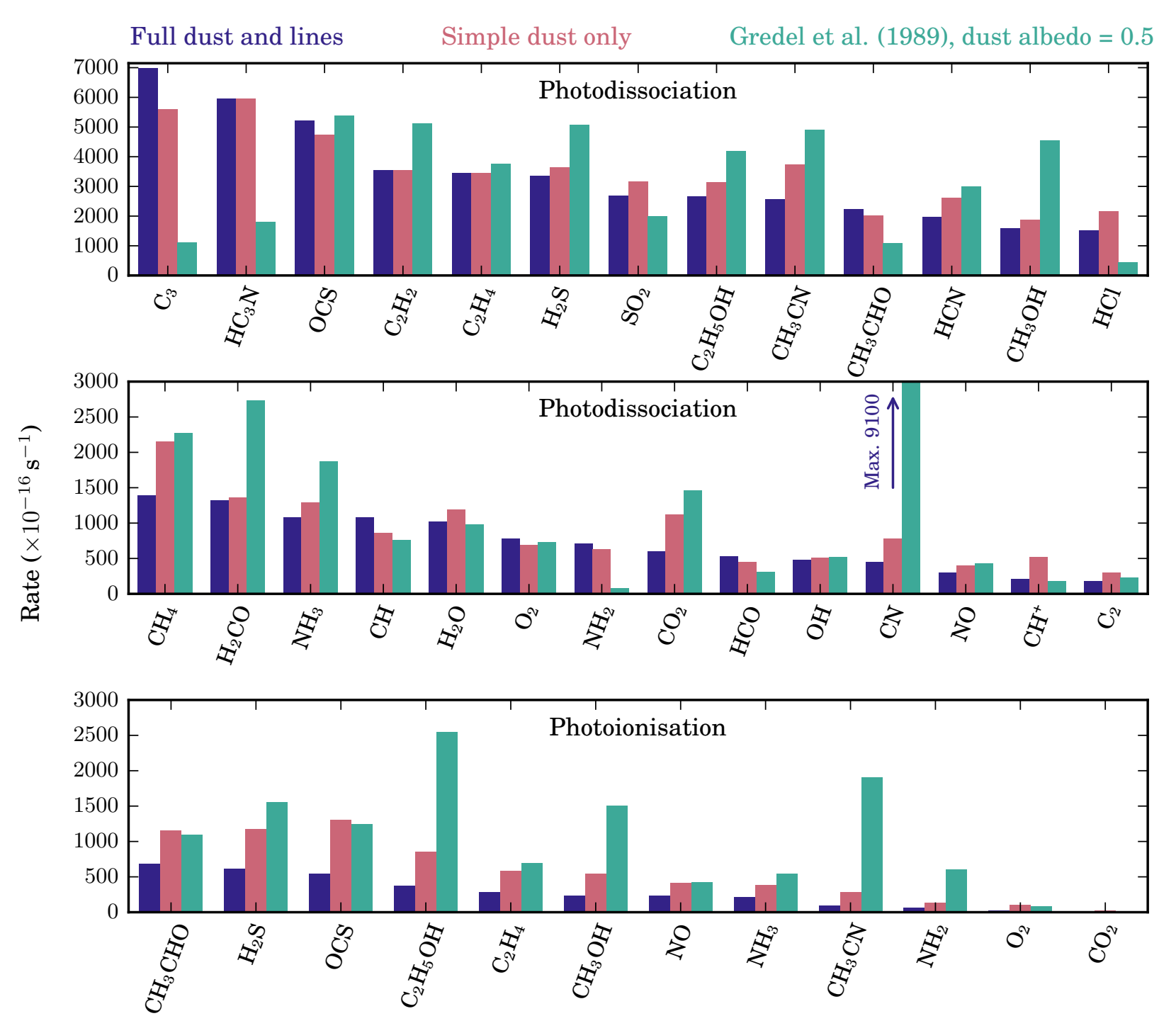

Fig. 33. Molecular photodissociation and ionisation rates due to cosmic-ray-induced radiation. Shown for all species common to this data base and Gredel et al. (1989). Full dust and lines: including all available shielding cross sections. Simple dust only: no molecular or atomic shielding, wavelength independent dust extinction cross section $2 \times 10^{-21} \mathrm{~cm}^{2} \mathrm{H}^{-1}$ and an albedo of 0.5 . Gredel et al:: rates computed from the efficiencies of Gredel et al. (1989; Table 1, column "a”, ignoring reabsorption) following multiplication by $10^{-16}$.

rates calculated here should then be considered conditional on the assumed abundances. To test the severity of this assumption, neglecting line shielding entirely in favour of pure dust absorption increased the calculated photodissociation and ionisation rates by less than a factor of 2, apart from the self-shielding cases of $\mathrm{N}_{2}$ and $\mathrm{CO}$ that are treated in detail elsewhere (Gredel et al. 1987; Heays et al. 2014b).

\section{Further discussion}

\subsection{Effect of dust-grain properties on ultraviolet shielding}

The dominant absorber of ultraviolet radiation from interstellar, stellar, or cosmic-ray sources is dust. More radiation will be available for gas-phase photodestruction if the dust mass is reduced. The ratio of gas-mass to dust-mass adopted in our calculations, 124, is taken in line with the Milky Way dust model of Draine et al. ${ }^{17}$ (Draine 2003a), and is somewhat larger than the frequently-used value of 100 . This parameter is also estimated from observations of local group Galaxies, and found to vary between about 50 and 500 (Draine et al. 2007; Leroy et al. 2011), and on small scales within the Milky Way, in one case falling to 88 and rising above 200 within one star-forming region (Liseau et al. 2015), although any such determinations have large inherent uncertainties. Finally, low "metallicity" (using astronomical terminology) galaxies such as the Magellanic Clouds have higher gas-to-dust ratios by up to an order of magnitude (Roman-Duval et al. 2014).

The ultraviolet opacity of dust is also reduced by its coagulation into larger sizes (Mathis et al. 1977; Draine \& Lee 1984; van Dishoeck et al. 2006). This phenomena is observed (e.g., Li \& Lunine 2003; Kessler-Silacci et al. 2006; Testi et al. 2014) and predicted (e.g., Birnstiel et al. 2010) to occur in protoplanetary disks, at least up to $\mathrm{cm}$-sized grains, with

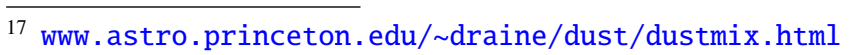


the larger grains settling to the midplane and drifting towards the central star. Disks also show additional complex spatial structures seen in small and large grains being influenced by planetary bodies or dynamical instabilities (e.g., Johansen et al. 2007; Andrews et al. 2011; Muto et al. 2012; van der Marel et al. 2013; Pinilla et al. 2015).

The dust shielding functions plotted in Figs. 27 are shifted to higher gas column densities when their ultraviolet opacity is reduced by grain growth. The scaling between $550 \mathrm{~nm}$ visual extinction, $A_{\mathrm{V}}$, and ultraviolet extinction also changes due to the wavelength-dependent variation of all dust grain optical properties when their size distribution and composition is modified (Mathis et al. 1977). van Dishoeck et al. (2006) simulated the dust optical properties of a large-grain populated protoplanetary disk, designed to best reproduce observations of the dust disk around HD 141569A (Li \& Lunine 2003; Jonkheid et al. 2006; van Dishoeck et al. 2006). This required the presence of dust grains as large as a few $\mu \mathrm{m}$ in diameter. Figure 26 compares the optical properties of this large-grain population with standard interstellar grains, showing a significantly-reduced ultraviolet absorption cross section (which matches interstellar grains at $550 \mathrm{~nm}$ ) and a somewhat increased albedo and forward scattering probability. These changes all act to increase the penetration of photodestructive radiation through the disk. We computed alternative shielding functions assuming a HD 141569A dust population and compare these with the interstellar dust case in Fig. 27. The larger grains require nearly an order-of-magnitude greater column of dust mass before shielding effectively. This leads to correspondingly smaller $\gamma_{\mathrm{E}_{2}}$-factors in Eq. (13), about 0.5 for most molecules, as listed in Tables 18 and 19, as opposed to values of about 1.5 assuming interstellar type dust.

Grain growth will also increase photodissociation and ionisation rates in a cosmic-ray induced UV field. This may be significant in the midplane of a protoplanetary disk due to aggregation of dust in the high-density environment and the gravitational settling of large grains (D'Alessio et al. 2001). The reduced competitiveness of dust grains in the absorption of UV photons will then affect the chemistry of gas-phase molecules (Chaparro Molano \& Kamp 2012).

\subsection{Effect of temperature on molecular cross sections}

Most laboratory measurements are recorded at room temperature, whereas many theoretical calculations do not include any ground state excitation whatsoever, simulating a low temperature of $<10 \mathrm{~K}$ with $v=0$ and $J=0$. Fortunately, the difference between cross sections appropriate for interstellar or atmospheric excitation temperatures $(10$ to about $1000 \mathrm{~K})$ and the available measurements and calculations is largely obliterated by the wavelength integration of Eq. (1). At temperatures up to a few hundred $\mathrm{K}$, multiple rotational levels besides $J=0$ will be excited, in most cases broadening the cross section slightly without changing its integrated value (e.g., Wu et al. 2000; Miyake et al. 2011; Li et al. 2013). At higher temperatures, such as encountered in protoplanetary disks and exoplanet atmospheres, vibrational levels of the ground electronic state start to be excited but as long as $v \leq 2$ this again only results in a small wavelength redistribution of the integrated cross section. Such a change in cross section shape is unlikely to affect ISRF photodestruction rates but could significantly alter rates in a cool stellar radiation field if the cross section shifts in or out of the maximum of the stellar flux. This effect is particularly prominent for the case of $\mathrm{CO}_{2}$ at long wavelengths, as demonstrated by Venot et al. (2013).

Li et al. (2013) find the $1000 \mathrm{~K}$ ISRF photodissociation rate of $\mathrm{N}_{2}$ to be only $15 \%$ larger than at $10 \mathrm{~K}$ but a large temperature dependence for its self-shielding and shielding by $\mathrm{H}_{2}$, with factor-of-10 decreases of shielding effectiveness between 10 and $1000 \mathrm{~K}$. A factor-of-two temperature variability of the $\mathrm{N}_{2}$ cosmic-ray induced photodissociation rate was also noted (Heays et al. 2014b) between 10 and $300 \mathrm{~K}$. The temperature sensitivity of $\mathrm{N}_{2}$, and also $\mathrm{CO}$ (Visser et al. 2009) and $\mathrm{H}_{2}$ (Sternberg et al. 2014), arises from their rotational-line dominated spectra where linewidths are set mostly by the Doppler broadening. A higher kinetic temperature leads to more Doppler broadening and a lesser cross section at the line centres, reducing the effectiveness of self-shielding. And, a higher excitation temperature spreads the cross section over a greater number of rotational lines, also impeding self-shielding. A more subtle effect occurs for some predissociating molecules where the dissociation efficiency, $\eta^{\mathrm{d}}$, increases with excitation temperature (this can happen because of increased centrifugal mixing of excited states for faster rotation molecules, e.g., Lewis et al. 2005; Eidelsberg et al. 2014).

\subsection{Uncertainty of calculated line positions and widths}

As noted in Sect. 3.3, theoretical cross sections are sometimes calculated as vertical excitations from the ground to one or more excited states, with each electronic transition summarised by a single line (e.g., van Hemert \& van Dishoeck 2008). Then the assumption of a linewidth is required. The ISRF photodestruction rates calculated in Sect. 5 are not sensitive to the precise choice of width, however, the rates of cosmic-ray induced processes in Sect. 7 as well as rates for highly structured radiation fields such as Lyman- $\alpha$ are somewhat sensitive. For the cosmicray induced case, experimentation with synthetic lines suggests that calculated rates averaged over 10 lines and assuming $1 \mathrm{~nm}$ linewidths will be accurate to within about a factor of 2 should the real linewidths fall in the range 0.1 to $10 \mathrm{~nm}$.

Another test was performed to determine the sensitivity of cosmic-ray induced photodissociation rates to uncertain vertical excitation energies. Rates were calculated for all molecules in our database that include vertical transitions (primarily those calculated by van Hemert \& van Dishoeck 2008) in a Monte Carlo simulation. For this, the wavelength positions of all transitions were repeatedly and independently adjusted by an amount falling within a uniform probability distribution bounded by the assumed vertical-transition energy uncertainty $\pm 0.2 \mathrm{eV}( \pm 3.6 \mathrm{~nm}$ at $150 \mathrm{~nm}$ ). Each line has an assumed intrinsic FWHM of $1 \mathrm{~nm}$. In this way, the possible range of overlap between absorption features and cosmic-ray induced emission lines was sampled. The resultant distribution of photodissociation rates for three molecules showing quite different sensitivities is shown in Fig. 34. Line-position uncertainty does not affect the photodissociation rate for $l-\mathrm{C}_{4}$ because the calculated absorption for this molecule occurs longwards of $170 \mathrm{~nm}$ and in the continuum part of the cosmic-ray induced spectrum. Whereas, the photodissociation of $l-\mathrm{C}_{3} \mathrm{H}$ is sensitive to the overlap of several of its transitions near to the strong $\mathrm{H}_{2}$ emission lines around $160 \mathrm{~nm}$. The proximity of $\mathrm{C}_{2} \mathrm{H}$ vertical transition to the Lyman- $\alpha$ wavelength means a small uncertainty in this transition energy leads to a large variation in photodissociation rate. All other molecules tested have distributions with widths falling within the range of Fig. 34, and these were considered when assigning the rate uncertainties listed in Table 20. 

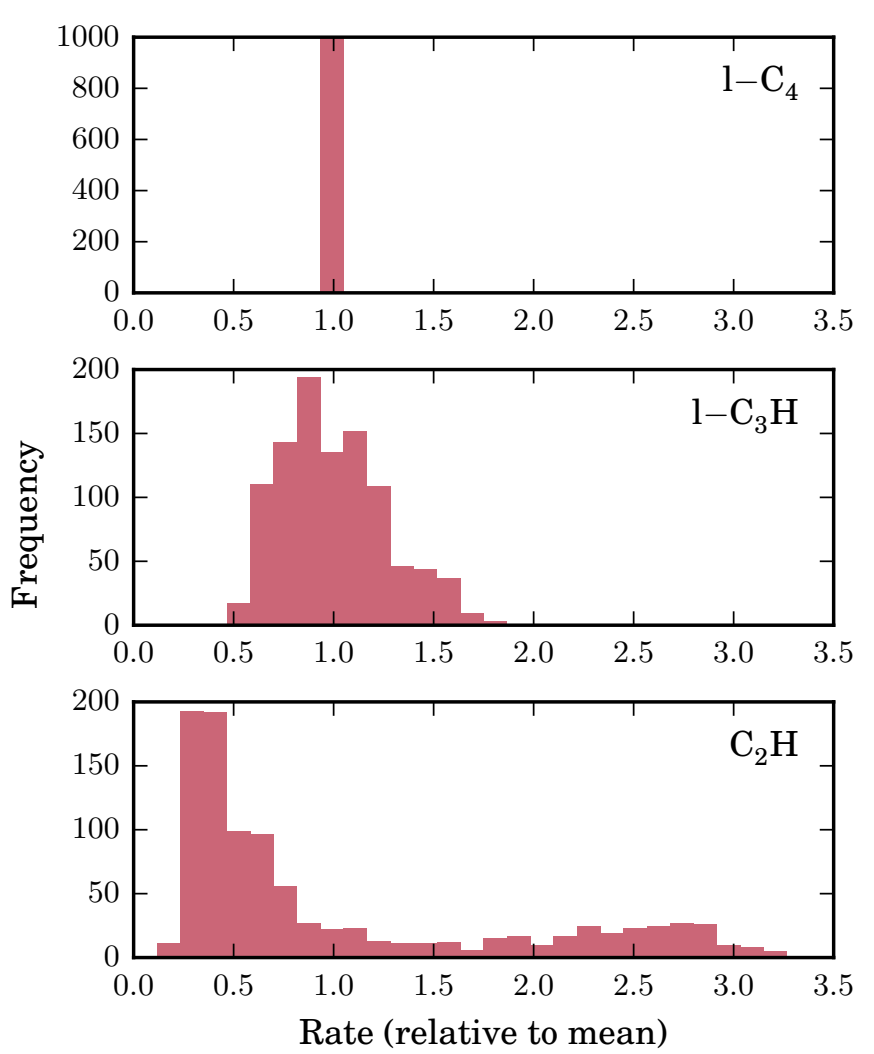

Fig. 34. Distribution of Monte Carlo simulated cosmic-ray-induced photodissociation rates of three molecules. Normalised to the mean of the distribution.

\subsection{Effect of unresolved structure on molecular cross sections}

The spectral resolution of laboratory equipment used to record photoabsorption cross sections is sometimes insufficient to completely resolve molecular vibrational-rotational structure. For example, our assembly of $\mathrm{H}_{2} \mathrm{O}$ data incorporates very highresolution measurements of Fillion et al. (2003), Fillion et al. (2004) and taken from the CfA VUV database ${ }^{18}$, with others that do not resolve the rotational spectrum (Chan et al. 1993b; Mota et al. 2005).

A comparison of $\mathrm{H}_{2} \mathrm{O}$ spectra is plotted in Fig. 35, all recorded at room temperature. The apparent changes between spectra are significant despite their integrated cross sections being essentially the same. For example, the apparent peak cross section of the vibrational band near $111.5 \mathrm{~nm}$, whose assignment is discussed in Fillion et al. (2004), varies by an order of magnitude. This peak-value difference is still a factor of three when comparing the two highest resolution cross sections available, CfA spectra with FWHM $0.0015 \mathrm{~nm}$ and those recorded by Fillion et al. (2004) with resolution 0.0025 to $0.005 \mathrm{~nm}$ FWHM.

In Table 21 we list photodissociation rates for $\mathrm{H}_{2} \mathrm{O}$ calculated using the highest-resolution data and neglecting this in favour of less-resolved cross sections (which still cover the entire wavelength range). In most cases, any difference is negated by the smoothing effect of wavelength integration and because the continuum part of the $\mathrm{H}_{2} \mathrm{O}$ cross section dominates the combined cross section of lines, despite their large maxima. The photodissociation rate due to Lyman- $\alpha$ dominated radiation is sharply

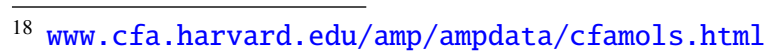

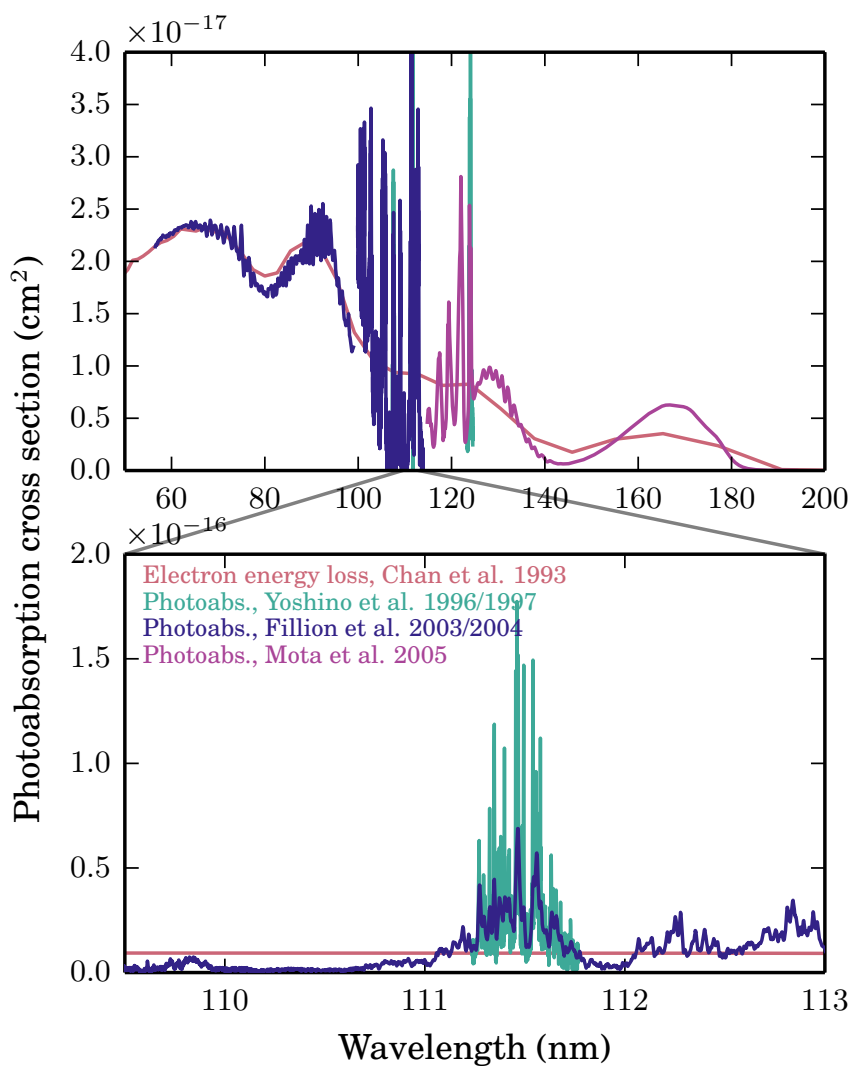

Fig. 35. $\mathrm{H}_{2} \mathrm{O}$ photoabsorption cross section measured by low-resolution electron energy-loss spectroscopy and by direct higher-resolution photoabsorption measurements.

reduced, however, when only the lowest resolution data set is used, because of the under resolution of a vibrational band near the Lyman- $\alpha$ wavelength.

The photodissociation cross sections of $\mathrm{CO}, \mathrm{N}_{2}$, and $\mathrm{H}_{2}$ have no continuum absorption at all and will be sensitive to insufficient experimental resolving power. For this reason, these molecules are treated here with models that recreate each absorption line and its lineshape from experimental data, but without the influence of experimental instrumental broadening (Visser et al. 2009; Li et al. 2013; Abgrall et al. 1993c). We can then directly test their sensitivity to the resolution-dependent phenomenon of self-shielding by broadening their cross sections though convolution with Gaussian functions of increasingly greater width.

The results of such a test are shown in Fig. 36, the shielding effect decreases significantly (shielding function approaches unity) when absorption lines of $\mathrm{N}_{2}, \mathrm{CO}$, and $\mathrm{H}_{2}$ are artificially broadened. From this figure, even the use of a $0.005 \mathrm{~nm}$ resolution cross section may incompletely-resolves and underestimate the self-shielding effect for a line-dominated molecular cross section spectrum.

Most species in our database are sufficiently continuumdominated that any under-resolution will not influence their calculated photodestruction rates. For example, we also systematically broaden the highest-resolution experimental spectrum of $\mathrm{H}_{2} \mathrm{O}$ and find a negligible effect in Fig. 36. The experimentallydetermined cross section of NO may benefit from the measurement of a higher resolution cross section between 130 to $165 \mathrm{~nm}$, particular if this molecule can attain a high column density in 
Table 21. Photodissociation rate of $\mathrm{H}_{2} \mathrm{O}$.

\begin{tabular}{|c|c|c|c|c|}
\hline $\begin{array}{c}\text { Data sources } \\
\text { (cf. Sect. 4.3.33 and Fig. 35) }\end{array}$ & $\begin{array}{l}\text { Max. resolution }^{a} \\
(\mathrm{~nm} \text { FWHM })\end{array}$ & $\begin{array}{l}\text { ISRF rate }^{b} \\
\left(\mathrm{~s}^{-1}\right)\end{array}$ & $\begin{array}{l}\text { Lyman- } \alpha \text { rate } \\
\qquad\left(\mathrm{s}^{-1}\right)\end{array}$ & $\begin{array}{c}\text { Cosmic-ray induced } \\
\text { rate }^{c}\left(\mathrm{~s}^{-1}\right)\end{array}$ \\
\hline All data & 0.0015 & $7.7 \times 10^{-10}$ & $2.4 \times 10^{-09}$ & $9.8 \times 10^{-14}$ \\
\hline Excluding CfA molecular database data & 0.0025 & $7.7 \times 10^{-10}$ & $2.4 \times 10^{-09}$ & $9.8 \times 10^{-14}$ \\
\hline Also excluding Fillion et al. & 0.075 & $8.3 \times 10^{-10}$ & $2.4 \times 10^{-09}$ & $10.0 \times 10^{-14}$ \\
\hline Also excluding Mota et al. (2005) & 10 & $8.4 \times 10^{-10}$ & $1.3 \times 10^{-09}$ & $8.4 \times 10^{-14}$ \\
\hline
\end{tabular}

Notes. These are calculated from subsets of the available experimental photodissociation cross sections. ${ }^{(a)}$ The maximum resolution within the combined data set. ${ }^{(b)}$ Calculated as in Sect. 5. ${ }^{(c)}$ Calculated as in Sect. 7.

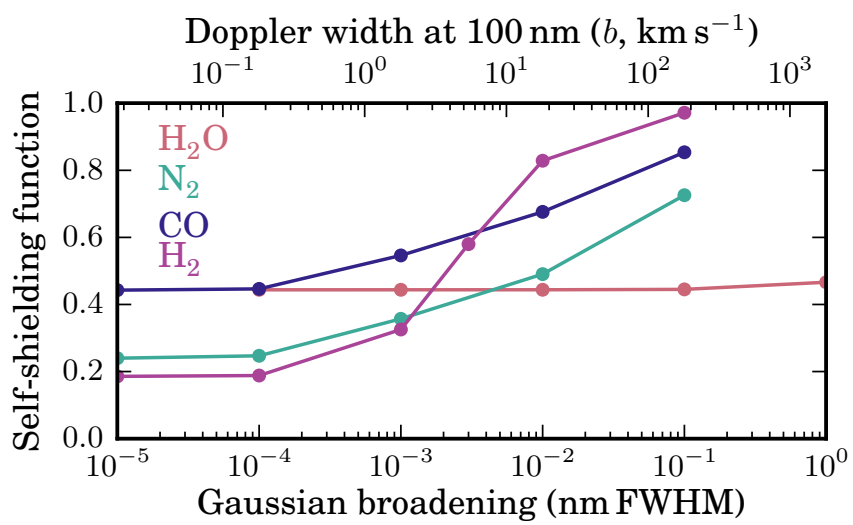

Fig. 36. ISRF self-shielding factors. Shown for four molecules after convolution of their photodissociation cross sections by Gaussian functions of increasing width (equivalent Doppler widths are also shown). For these calculations, a column density of $10^{15} \mathrm{~cm}^{-2}$ was assumed for $\mathrm{N}_{2}, \mathrm{CO}$ and $\mathrm{H}_{2}$; and $10^{17} \mathrm{~cm}^{-2}$ for $\mathrm{H}_{2} \mathrm{O}$.

an atmosphere. Some radical species, for example, $\mathrm{NH}_{2}$ or $\mathrm{C}_{2}$, likely also have unquantified sharply resonant features.

Turbulent Doppler broadening will actually reduce selfshielding in the way that experimental under-resolution mimics. This is shown in Fig. 36 where a Doppler width of $10 \mathrm{kms}^{-1}$ is significant at least for the case of $\mathrm{H}_{2}$. Larger Doppler widths are unlikely to occur in astrochemical environments except in locally shocked regions.

\subsection{Isotopic effects}

Isotopic substitutions of one or more atoms within a molecule alter its rotational inertia and the reduced masses of its vibrational modes (e.g., Herzberg 1989). The resultant shifts in rotationalvibrational energy levels may be different for ground and excited states so that the wavelengths of lines in its photoabsorption spectrum also shift.

For diatomic molecules, the largest shifts are expected when deuterating $\mathrm{H}_{2}$ to form $\mathrm{HD}$, a reduced mass (in atomic units) increase from $(1 \times 1) /(1+1)$ to $(1 \times 2) /(1+2)$. The resulting vibrational-level shifts for the astrophysically-important $B^{1} \Sigma_{u}^{+}$ and $C^{1} \Pi_{u}$ states of $\mathrm{H}_{2}$ can be as large as $1300 \mathrm{~cm}^{-1}$, equivalent to a $0.8 \mathrm{~nm}$ difference between the absorption line wavelengths of $\mathrm{H}_{2}$ and $\mathrm{HD}$. This shift is large enough that $\mathrm{H}_{2}$ no longer shields $\mathrm{HD}$, so that the photodissociation rate of HD with depth into a cloud is much larger than that of $\mathrm{H}_{2}$ (Spitzer et al. 1973; Black \& Dalgarno 1977). Isotopic substitution of a heavier element leads to smaller shifts. For example, the substitution of the minor isotope ${ }^{15} \mathrm{~N}$ into molecular nitrogen, $\mathrm{N}_{2}$, leads

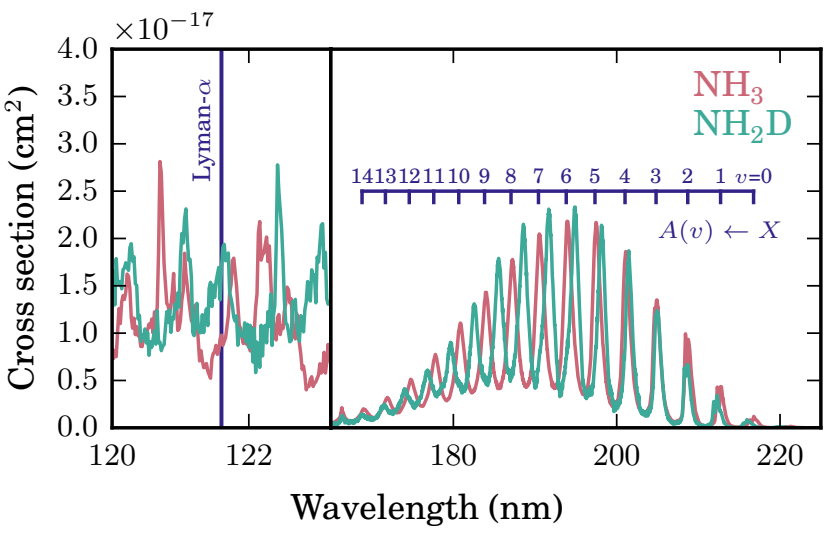

Fig. 37. Long-wavelength and near-Lyman- $\alpha$ parts of the $\mathrm{NH}_{3}$ and $\mathrm{NH}_{2} \mathrm{D}$ photoabsorption cross sections. The source of the $\mathrm{NH}_{3}$ cross section is discussed in Sect. 4.3.46 and the $\mathrm{NH}_{2} \mathrm{D}$ data are taken from two sources (Wu et al. 2007; Cheng et al. 2006). Also indicated is the wavelength of Lyman- $\alpha$ emission and the vibrational progression of absorption into the excited $\tilde{A}$ electronic state of $\mathrm{NH}_{3}$.

to changes in energy levels and absorption line wavelengths of at most $200 \mathrm{~cm}^{-1}$ and $0.15 \mathrm{~nm}$, respectively. This is still more than enough to maintain the self-shielding phenomenon for $\mathrm{N}_{2}$, but reduce the mutual shielding of ${ }^{14} \mathrm{~N}^{15} \mathrm{~N}$ by ${ }^{14} \mathrm{~N}_{2}$ (Heays et al. 2014b).

The deuteration of $\mathrm{NH}_{3}$ to $\mathrm{NH}_{2} \mathrm{D}$ leads to wavelength shifts of its $\tilde{A} \leftarrow \tilde{X}$ absorption bands, shown in Fig. 37, with larger shifts for higher vibrational levels of the $\tilde{A}$ state. Most important is the isotope-induced shift of higher-energy excited states into resonance with the Lyman- $\alpha$ emission line. The nearly two-times larger cross section of $\mathrm{NH}_{2} \mathrm{D}$ at $121.6 \mathrm{~nm}$ leads directly to an increased photodissociation rate of this species in a Lyman- $\alpha$ dominated radiation field.

The Lyman- $\alpha$ resonance of $\mathrm{C}_{2} \mathrm{H}_{2}$ is even more critical than for $\mathrm{NH}_{3}$, as shown in Fig. 23. A small isotopic shift following deuteration will lower the dissociation rate of $\mathrm{C}_{2} \mathrm{HD}$ in a Lyman$\alpha$ dominated radiation field by a factor of 10 . This reduction is in fact indicated from laboratory action spectroscopy of $\mathrm{C}_{2} \mathrm{H}_{2}$ and $\mathrm{C}_{2} \mathrm{HD}$ (Löffler et al. 1996; Wang et al. 1997).

The photodissociation efficiency or branching may also be sensitive to isotopic substitution. For example, the deuteration of water to form HDO introduces the possibility of preferential branching to form $\mathrm{OH}$ or OD photodissociation fragments and imposing a significant influence on the ratios of $\mathrm{H}_{2} \mathrm{O}$, $\mathrm{HDO}$, and $\mathrm{D}_{2} \mathrm{O}$ found in interstellar space, comets, and terrestrially (Caselli \& Ceccarelli 2012; van Dishoeck et al. 2013). The propensity for H-OD fission is determined in laboratory 
Table 22. Photodissociation branching of $\mathrm{H}_{2} \mathrm{O}$ and $\mathrm{NH}_{3}$.

\begin{tabular}{|c|c|c|c|c|c|c|}
\hline Radiation field $^{a}$ & Rate $^{b}$ & Frac. $^{c}$ & Unc. $^{d}$ & Rate & Frac. & Unc. \\
\hline & \multicolumn{3}{|c|}{$\mathrm{H}_{2} \mathrm{O} \rightarrow \mathrm{OH}+\mathrm{H}$} & \multicolumn{3}{|c|}{$\mathrm{H}_{2} \mathrm{O} \rightarrow \mathrm{O}+2 \mathrm{H} / \mathrm{H}_{2}$} \\
\hline ISRF & $5.9 \times 10^{-10}$ & 0.77 & B & $1.8 \times 10^{-10}$ & 0.23 & B \\
\hline Mathis' 83 & $4.0 \times 10^{-10}$ & 0.75 & $\mathrm{~B}$ & $1.3 \times 10^{-10}$ & 0.25 & B \\
\hline Black Body $4000 \mathrm{~K}$ & $1.6 \times 10^{-10}$ & 0.99 & $\mathrm{~A}$ & $2.3 \times 10^{-12}$ & 0.01 & $\mathrm{C}$ \\
\hline Black Body $10000 \mathrm{~K}$ & $4.4 \times 10^{-10}$ & 0.93 & $\mathrm{~A}$ & $3.3 \times 10^{-11}$ & 0.07 & B \\
\hline Lyman- $\alpha$ & $1.8 \times 10^{-09}$ & 0.74 & A & $6.2 \times 10^{-10}$ & 0.26 & A \\
\hline Solar & $2.0 \times 10^{-10}$ & 0.88 & A & $2.7 \times 10^{-11}$ & 0.12 & B \\
\hline TW-Hydra & $1.2 \times 10^{-09}$ & 0.76 & A & $4.0 \times 10^{-10}$ & 0.24 & A \\
\hline \multirow[t]{2}{*}{ Cosmic-ray induced } & $7.6 \times 10^{-14}$ & 0.77 & $\mathrm{~B}$ & $2.2 \times 10^{-14}$ & 0.23 & $\mathrm{~B}$ \\
\hline & \multicolumn{3}{|c|}{$\mathrm{NH}_{3} \rightarrow \mathrm{NH}_{2}+\mathrm{H}$} & \multicolumn{3}{|c|}{$\mathrm{NH}_{3} \rightarrow \mathrm{NH}+2 \mathrm{H} / \mathrm{H}_{2}$} \\
\hline ISRF & $8.3 \times 10^{-10}$ & 0.58 & B & $6.1 \times 10^{-10}$ & 0.42 & B \\
\hline Mathis'83 & $5.6 \times 10^{-10}$ & 0.57 & B & $4.3 \times 10^{-10}$ & 0.43 & B \\
\hline Black Body $4000 \mathrm{~K}$ & $3.6 \times 10^{-09}$ & 1.00 & A & $2.3 \times 10^{-13}$ & 0.00 & $\mathrm{C}$ \\
\hline Black Body $10000 \mathrm{~K}$ & $1.6 \times 10^{-09}$ & 0.95 & A & $9.0 \times 10^{-11}$ & 0.05 & B \\
\hline Lyman- $\alpha$ & $6.6 \times 10^{-11}$ & 0.05 & B & $1.3 \times 10^{-09}$ & 0.95 & A \\
\hline Solar & $4.0 \times 10^{-09}$ & 0.99 & $\mathrm{~A}$ & $5.6 \times 10^{-11}$ & 0.01 & $\mathrm{C}$ \\
\hline TW-Hydra & $4.9 \times 10^{-10}$ & 0.35 & A & $9.1 \times 10^{-10}$ & 0.65 & A \\
\hline Cosmic-ray induced & $3.9 \times 10^{-14}$ & 0.39 & B & $6.2 \times 10^{-14}$ & 0.61 & B \\
\hline
\end{tabular}

Notes. ${ }^{(a)}$ Radiation fields are defined and normalised as for Tables 18 and 20. ${ }^{(b)}$ In units of $\mathrm{s}^{-1}$. $^{(c)}$ Estimated branching fraction between all channels producing $\mathrm{OH}$ and $\mathrm{O}$ (or $\mathrm{NH}_{2}$ and $\mathrm{NH}$ ) regardless of excitation state or the chemical co-fragment. ${ }^{(d)}$ Estimated rate uncertainties: accurate to within $30 \%(\mathrm{~A})$, a factor of 2 (B), a factor of $10(\mathrm{C})$.

measurements at a few UV wavelengths (e.g., Shafer et al. 1989; Plusquellic et al. 1998) and theoretical calculations (e.g., Engel \& Schinke 1988; Zhang et al. 1989; Zhou \& Xie 2015), together indicating a wavelength and temperature dependent ratio of OD or $\mathrm{OH}$ fragments between 2 and 16 (or higher).

\subsection{Photodissociation branching of $\mathrm{H}_{2} \mathrm{O}$ and $\mathrm{NH}_{3}$}

We estimated the wavelength dependent branching ratios of $\mathrm{H}_{2} \mathrm{O}$ and $\mathrm{NH}_{3}$ into their main astrochemically-significant photodissociation products $\mathrm{OH}$ and $\mathrm{O}$, and $\mathrm{NH}_{2}$ and $\mathrm{NH}$, respectively. A discussion of these cross sections is given in Sects. 4.3.33 and 4.3.46, and the wavelength dependent partial cross sections shown in Figs. 16 and 17. We calculated the partial photodissociation rates generating these products when exposed to various kinds of interstellar and cosmic-ray-generated radiation fields, with results given in Table 22 . The uncertainty estimates provided in this table are a combination of the overall cross section uncertainties of Table 1 and an estimate of the branchingratio accuracy. Consideration was also made that minor branching channels will have larger fractional uncertainties than major ones.

The product branching ratios of both $\mathrm{H}_{2} \mathrm{O}$ and $\mathrm{NH}_{3}$ are well known at the Lyman- $\alpha$ wavelength, reasonably constrained at longer wavelengths, but poorly known shortwards of $121.6 \mathrm{~nm}$. Then, their ISRF and cosmic-ray-induced photodissociation rates are assigned a larger uncertainty than for other stellar radiation fields because these are approximately $40 \%$ controlled by radiation shorter than Lyman- $\alpha$. All other stellar rates are controlled by product branching ratios at Lyman- $\alpha$ or longwards.

The partial rates calculated here are in line with the previous version of the Leiden database for $\mathrm{NH}_{3}$ but lead to significantly greater production of atomic-O following the dissociation of $\mathrm{H}_{2} \mathrm{O}$ in the ISRF, $23 \%$ versus $6 \%$. This is due to the product branching calculations of (van Harrevelt \& van Hemert 2008) prompting the estimation of a higher $\mathrm{O}$ branching for wavelengths shorter than Lyman- $\alpha$. This assumption is however quite uncertain. The ratio of partial rates of $\mathrm{NH}_{3}$ products due to cosmic ray photodissociation is $\mathrm{NH}_{2} / \mathrm{NH}=0.6$ and has decreased relative to the calculation of Gredel et al. (1989), finding a ratio of 2.4

\section{Updated astrochemical reaction network}

The significance of the new and updated rates listed in Tables 18-20 are investigated by trialling a set of astrochemical models. These single-point time-dependent gas-phase models use the integration program of Walsh et al. (2009, 2010) to trace the chemical evolution of a range of atoms and molecules assuming a constant temperature, density, and visual extinction; and a set of initial abundances. For simplicity, these models are restricted to pure gas-phase chemistry, apart from the inclusion of grain-surface-mediated $\mathrm{H}_{2}$ formation, and neglect the selfshielding of $\mathrm{CO}$ and $\mathrm{N}_{2}$.

We adopt the RATE12 reaction network (McElroy et al. 2013) in a fiducial model, and then modify it by substituting or adding our newly-calculated photodissociation and photoionisation rates. This required the updating of 111 ISRF photodissociation and ionisation rates, and 82 cosmic-ray induced rates; and adding 40 new rates for species in the network that lacked one or more photodestruction processes. Alternative networks were also constructed that replace the ISRF rates with those appropriate for black-body fields of various temperatures, and the simulated flux from TW-Hydra, described in Sect. 2.

Two kinds of models were run, with their important parameters listed in Table 23. The translucent cloud model is useful for evaluating the effect of the updated ISRF photodestruction rates, and the dark cloud model for isolating the effects of the new 


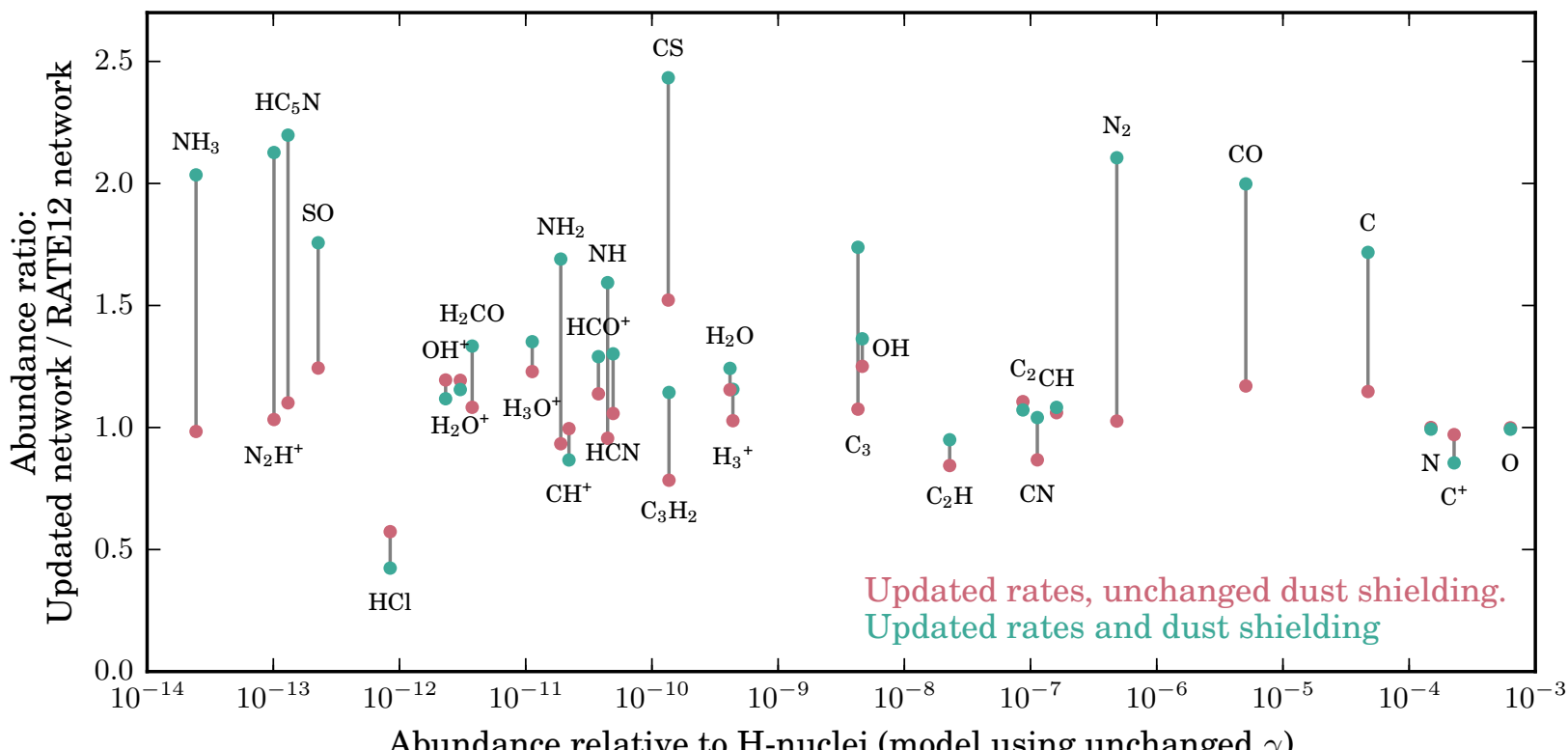

Fig. 38. Fractional change of chemical abundances in a translucent cloud model when substituting photodestruction rates of the RATE12 network with the newly-calculated rates. A comparison is made with updated models including the same $\gamma_{\exp }$ parameterisation as RATE12 for dust shielding, and with dust shielding calculated explicitly for each molecule. The plotted species are those listed in Tielens (2013, Table II).

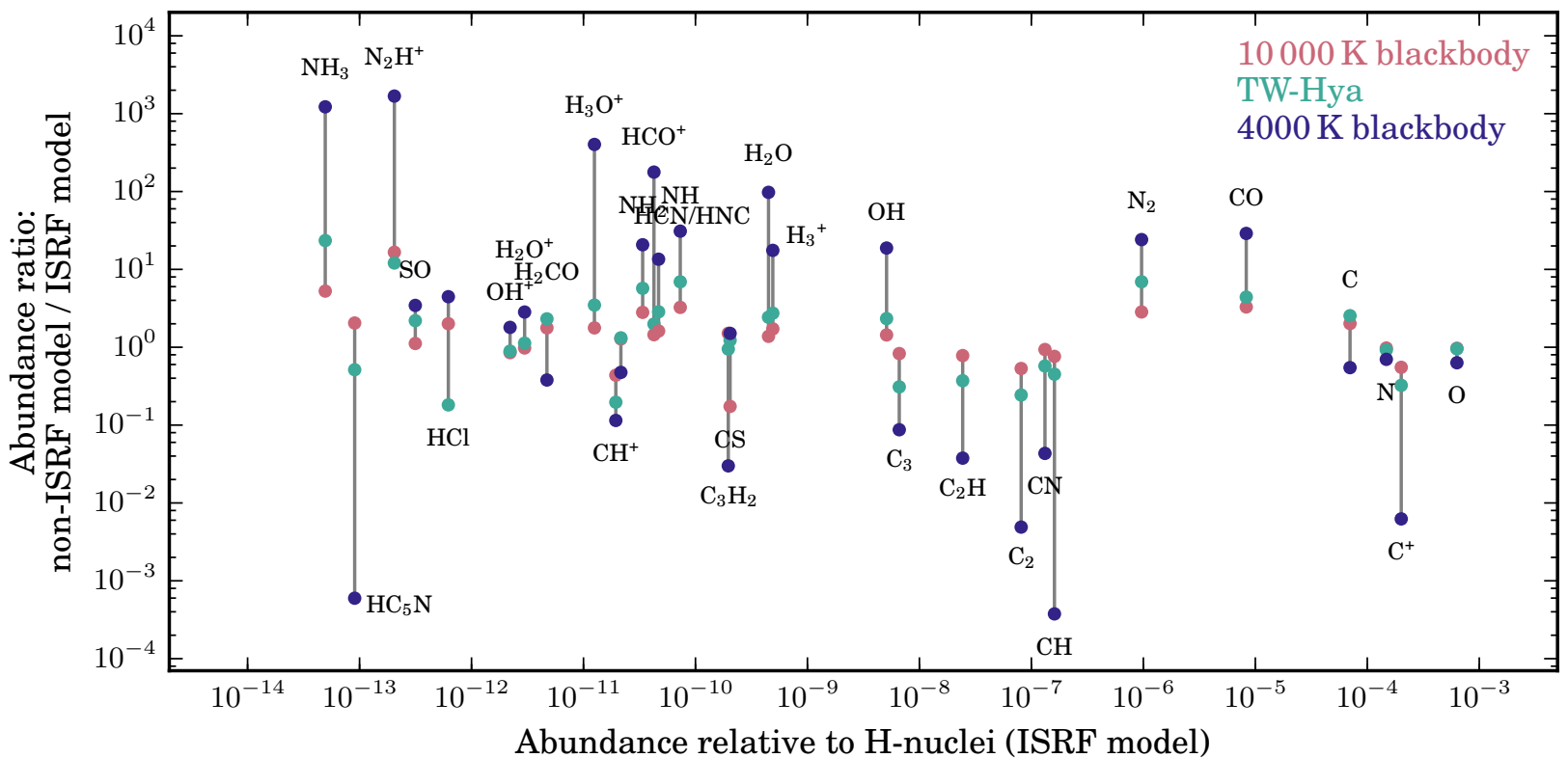

Fig. 39. Comparing the chemical abundances in a translucent cloud model assuming non-ISRF radiation fields. The plotted species are those listed in Tielens (2013, Table II).

cosmic-ray induced rates. We integrated the models until reaching chemical equilibrium, requiring about $10^{6}$ years of model time.

The effects of the updated rates on model abundances at $A_{\mathrm{V}}=1$ in a translucent cloud exposed to the ISRF are modest, with no abundance changes relative to the RATE12 model exceeding a factor of two. Trialling additional pairs of models after making order-of-magnitude variations of the temperature, density, and $A_{\mathrm{V}}$ in the translucent cloud model resulted in similar differences.
An explicit radiative transfer (or improved parameterisation) of dust shielding is as important as the new photodestruction rates in altering the chemical model output. The RATE12 network incorporates the $k_{0} \exp \left(-\gamma_{\exp } A_{\mathrm{V}}\right)$ depth-dependent photodestruction rates of van Dishoeck et al. (2006). The effect on model abundances of updating all photodestruction rates while retaining the RATE12 $\gamma_{\text {exp }}$ parameters is demonstrated in Fig. 38 for a selection of atoms and molecules with $A_{\mathrm{V}}=1$. Also shown is a model with updated rates and an explicit radiative-transfer calculation simulating their reduction at a depth of $1 A_{\mathrm{V}}$ (as in 


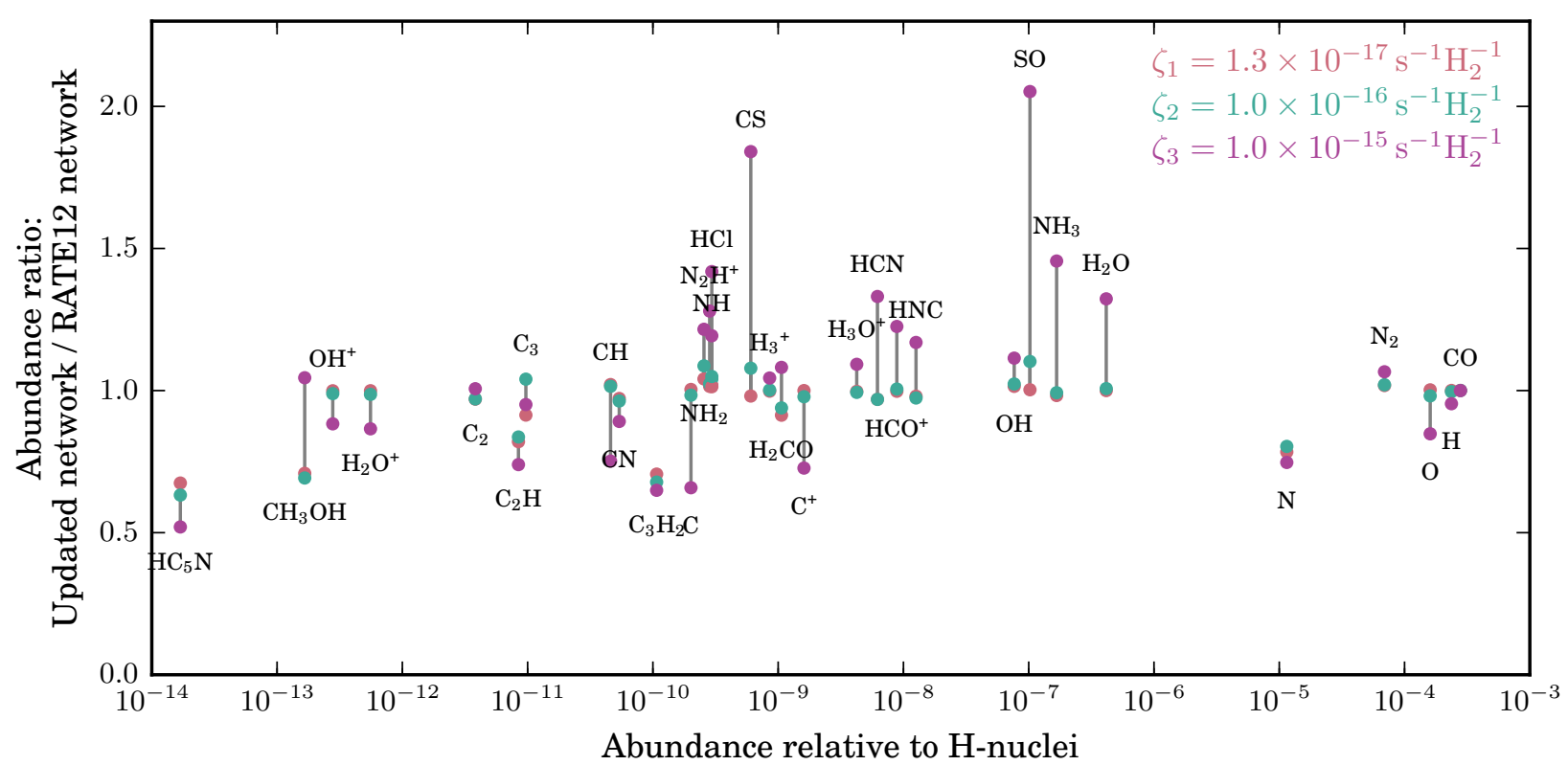

Fig. 40. Fractional change of chemical abundances in the dark-cloud model when substituting photodestruction rates of the RATE12 network with the newly-calculated rates. A comparison is made for models assuming increasing ionisation rate, $\zeta$. The plotted species are those listed in Tielens (2013, Table II).

Table 23. Physical parameters of interstellar cloud point models.

\begin{tabular}{ccc}
\hline \hline & Translucent & Dark \\
\hline Temperature $(\mathrm{K})$ & 100 & 10 \\
Density $\left(\mathrm{cm}^{-3}\right)$ & $10^{3}$ & $10^{4}$ \\
$A_{\mathrm{V}}\left(\mathrm{mag}^{3}\right)$ & 1 & 100 \\
$\zeta_{\mathrm{H}_{2}}\left(10^{-17} \mathrm{~s}^{-1} \mathrm{H}_{2}^{-1}\right)$ & 1.3 & $1.3-100$ \\
\hline
\end{tabular}

Sect. 6). The addition of updated dust shielding leads to an increased abundance for most species by up to a factor of two after chemical equilibrium is reached.

The $\gamma_{\exp }$ parameters used in RATE12 generally underestimate the shielding effect of a semi-infinite slab of dust at an extinction of 1 , and overestimate it at higher extinction, as exampled in Fig. 28. The explicit radiative transfer calculation then results in lower photodestruction rates and generally-higher abundances of molecules and neutral atoms at $1 A_{\mathrm{V}}$. The disadvantages of the $\gamma_{\exp }$ parameterisation can be largely avoided while retaining its computational efficiency by adopting the more realistic exponential-integral formulation (with $\gamma_{\mathrm{E}_{2}}$ parameters in Sect. 6.2).

Some species shown in Fig. 38 are affected by the updated rates and dust shielding but are not included in our current database of wavelength-dependent cross sections, for example, $\mathrm{N}_{2} \mathrm{H}^{+}$or $\mathrm{HC}_{5} \mathrm{~N}$. The modelled changes are then due to chemical formation and destruction routes involving species that we updated. The sensitivity of a chemical network to its input rates and the propagating influence of specific rates to unrelated model species is studied in the context of interstellar and atmospheric chemistry (e.g., Wakelam et al. 2010; Loison et al. 2015).

The wavelength dependence of our collected cross sections significantly alters the model output for non-ISRF ultraviolet radiation fields. Figure 39 summarises the equilibrium abundances calculated for 31 species known to compose interstellar clouds
(Tielens 2013, Table II). The variation of these abundances with radiation field is plotted relative to the abundances calculated in the ISRF. Substitution with a cooler $10000 \mathrm{~K}$ black-body radiation field increases the abundance of $\mathrm{CO}$ and $\mathrm{N}_{2}$ by factors of 4 and 20, respectively, because these molecules photodissociate at relatively short wavelengths only. The reduced occurrence of reactive $\mathrm{C}$-atoms then lowers the abundance of small carbon-containing molecules by a similar factor. On the other hand, nitrogen-containing species, for example, $\mathrm{NH}_{2}$ and $\mathrm{N}_{2} \mathrm{H}^{+}$, have increased abundances. This is due to their increased lifetime in the cooler ultraviolet flux and a formation route reliant on ion-molecule reactions with $\mathrm{N}_{2}$ and not its photodissociation (Walsh et al. 2015). Similar effects with somewhat larger magnitude follow from assuming the TW-Hydra radiation field, and with multiple-order-of-magnitude changes when assuming the extremely long-wavelength biased $4000 \mathrm{~K}$ black-body field. An extreme example is the increased abundance of $\mathrm{H}_{3} \mathrm{O}^{+}$, even though we did not include a direct photodestruction mechanism for this molecule in our model. The modelled increase is due to the decreased photoionisation of other species in the black-body radiation field, and a lowered electron abundance slowing the rate of $\mathrm{H}_{3} \mathrm{O}^{+}$dissociative recombination.

These differences are strongly-dependent on the total integrated flux of each radiation field, which we artificially normalised as is described in Sect. 2. However, an alternative scaling of the integrated flux will result in similarly divergent chemical abundances as pictured in Fig. 39 whenever photodissociation dominates molecular destruction, due to the wavelength-dependence of atomic and molecular cross sections: the abundances of small carbon-bearing molecules are reduced in cool radiation fields whereas nitrogen-containing species are enhanced.

Re-running the model while assuming weaker shielding from larger dust grains (with optical properties shown in Fig. 26) leads to significantly more photodestruction and a reduced population of stable molecules, for example, the abundance of $\mathrm{CO}$ is 
reduced by a factor of 30 , and $\mathrm{CN}$ by a factor of 10 . A very similar effect is achieved in our single-point steady-state model by retaining an interstellar dust opacity but reducing the model $A_{\mathrm{V}}$ from 1 to 0.2 . More sophisticated 1 - or multi-dimensional astrochemical models would likely distinguish between these effects, and could include the effects of $\mathrm{H}_{2}$-shielding and self-shielding, necessary for computing a self-consistent PDR model.

The dark cloud model was used to assess the effect of the new rates on cosmic-ray induced photodissociation and ionisation. After comparing models using the RATE12 network and updated cosmic-ray induced photodestruction rates, and assuming a primary ionisation rate of $\zeta=1.3 \times 10^{-17} \mathrm{~s}^{-1} \mathrm{H}_{2}^{-1}$, no change greater than a factor of 2 was found for any species with abundance greater than $10^{-14}$ relative to $\mathrm{H}_{2}$. Alternative models were run with the comic-ray induced ionisation rates of $\mathrm{H}_{2}$ and $\mathrm{H}$ and ultraviolet flux increased by a common factor. Model results corresponding to $\zeta=10^{-16}$ and $10^{-15} \mathrm{~s}^{-1} \mathrm{H}_{2}^{-1}$ are also shown in Fig. 40. Under the extreme model of cosmic-ray influence the effects of the updated rates calculated here are somewhat increased for some species, particularly $\mathrm{CH}_{3} \mathrm{OH}, \mathrm{CS}, \mathrm{SO}$, and $\mathrm{NH}_{3}$. Even in this case other sources of uncertainty in dark cloud chemical models may overshadow the abundance changes engendered by our updated rates.

\section{Summary}

A new collection of photodissociation and photoionisation cross sections was assembled for atoms and molecules of astrochemical interest, with uncertainty estimates. These data are used to calculate photodissociation and photoionisation rates in the ISRF and other radiation fields, including Lyman- $\alpha$ dominated radiation and a cosmic-ray induced ultraviolet flux. The majority of photodissociation and ionisation rates agree within 30\% when compared with other recent compilations (van Dishoeck et al. 2006; Huebner \& Mukherjee 2015; Gredel et al. 1989; where these include comparable cross sections), with some important differences. The reduction of these rates in shielded regions was calculated as a function of the dust, molecular and atomic hydrogen, neutral C, and self-shielding columns. Dust opacity is generally the most important shielding effect but a comparable influence from other forms of shielding was found for some molecules, particularly if grain growth has reduced the ultraviolet absorption cross section of the dust population.

Various sensitivities of the calculated rates to the experimental and theoretical data they are derived from, or the astrophysical environments where they are applied, is given. For most molecules, the under-resolution of resonant photoabsorption lines in experimental cross sections, small errors in the excitation electronic energies and linewidths in theoretical calculations, changes in excitation temperature, or isotopic substitution will not dramatically affect their astrochemical photodestruction. Exceptions occur for some molecules that show a high degree of sensitivity to these details, particularly when their cross sections feature a maximum near the Lyman- $\alpha$ wavelength.

Some tests of the new rates in simple astrochemical models show sensitivity to the updated rates up to a factor of two for molecules important in translucent and dark interstellar clouds. Additional sensitivity was shown to an improved dust-shielding parameterisation scheme that better matches the attenuation of absorbed and scattered UV light through a slab-model interstellar cloud.

The intention is to provide, along with precomputed rates and shielding functions, as detailed as possible wavelengthdependent cross sections that are suitable for use in astrochemical models of interstellar and circumstellar material that require specific treatments of photodissociation and photoionisation. That is, due to spatial and time variance of the ultraviolet radiation flux, temperature, density, turbulence, and dust optical properties, which cannot be easily or comprehensively parameterised.

The cross sections and derived data for a total of 102 molecules and atoms are available from the Leiden database $^{19}$. These are provided in both a binary format, explicitly including all cross section features on a dense wavelength grid, and sparser text format that is more suitable for rapid calculation in a continuum radiation field like the ISRF. This new database extends its previous version by the addition of 9 new species and cross sections updates for 60 more.

Acknowledgements. Thanks to Roland Gredel for provision of his cosmic-ray induced UV spectrum, Catherine Walsh for the use of her single-point astrochemical model program, and Marc van Hemert for ab initio calculations of CN. Astrochemistry in Leiden is supported by the European Union A-ERC grant 291141 CHEMPLAN, by The Netherlands Research School for Astronomy (NOVA), by a Royal Netherlands Academy of Arts and Sciences (KNAW) professor prize, and the Dutch astrochemistry network (DAN) from The Netherlands Organisation for Scientific Research (NWO) under grant 648.000.002. Thanks also to the anonymous referee for many useful comments, particularly regarding the use of exponential-integral functions to represent UV-shielding by dust grains.

\section{References}

Abgrall, H., \& Roueff, E. 2006, A\&A, 445, 361

Abgrall, H., Roueff, E., Launay, F., Roncin, J. Y., \& Subtil, J. 1993a, A\&AS, 101,273

Abgrall, H., Roueff, E., Launay, F., Roncin, J. Y., \& Subtil, J. 1993b, A\&AS, 101,323

Abgrall, H., Roueff, E., Launay, F., Roncin, J. Y., \& Subtil, J. L. 1993c, J. Mol. Spectr., 157, 512

Abgrall, H., Roueff, E., Launay, F., \& Roncin, J.-Y. 1994, Can. J. Phys., 72, 856

Abgrall, H., Roueff, E., Liu, X., \& Shemansky, D. E. 1997, ApJ, 481, 557

Abgrall, H., Roueff, E., Liu, X., Shemansky, D. E., \& James, G. K. 1999, J. Phys. B, 32, 3813

Abgrall, H., Roueff, E., \& Drira, I. 2000, A\&AS, 141, 297

Ackerman, M. 1971, in Mesospheric models and related experiments, ed. G. Fiocco, Astrophys. Space Sci. Lib., 25, 149

Ádámkovics, M., Glassgold, A. E., \& Najita, J. R. 2014, ApJ, 786, 135

Alexander, M. H., Li, X., Liyanage, R., \& Gordon, R. J. 1998, Chem. Phys., 231, 331

Andrews, S. M., Wilner, D. J., Espaillat, C., et al. 2011, ApJ, 732, 42

Angel, G. C., \& Samson, J. A. R. 1988, Phys. Rev. A, 38, 5578

Archer, L., Stark, G., Smith, P., et al. 2013, J. Quant. Spectr. Radiat. Transf., 117, 88

Ardila, D. R., Basri, G., Walter, F. M., Valenti, J. A., \& Johns-Krull, C. M. 2002a, ApJ, 566, 1100

Ardila, D. R., Basri, G., Walter, F. M., Valenti, J. A., \& Johns-Krull, C. M. 2002b, ApJ, 567, 1013

Ashfold, M. N. R., Nahler, N. H., Orr-Ewing, A. J., et al. 2006, Phys. Chem. Chem. Phys., 8, 26

Au, J., Cooper, G., Burton, G., Olney, T., \& Brion, C. 1993, Chem. Phys., 173, 209

Babb, J. F. 2015, ApJS, 216, 21

Backx, C., \& Vanderwiel, M. 1975, J. Phys. B, 8, 3020

Backx, C., Wight, G. R., \& der Wiel, M. J. V. 1976, J. Phys. B, 9, 315

Bahou, M., Chung, C.-Y., Lee, Y.-P., et al. 2001, ApJ, 559, L179

Baltayan, P., \& Nedelec, O. 1979, J. Chem. Phys., 70, 2399

Barthel, M., Flesch, R., Rühl, E., \& McLaughlin, B. M. 2015, Phys. Rev. A, 91, 013406

Bates, D. R., \& Spitzer, Jr., L. 1951, ApJ, 113, 441

Bauschlicher, C. W., \& Langhoff, S. R. 1988, J. Chem. Phys., 89, 2116

Bauschlicher, C. W., \& Partridge, H. 1995, Chem. Phys. Lett., 239, 246

Bautista, M. 1997, A\&AS, 122, 167

Becker, S., Braatz, C., Lindner, J., \& Tiemann, E. 1995, Chem. Phys., 196, 275

Belau, L., Wheeler, S. E., Ticknor, B. W., et al. 2007, J. Am. Chem. Soc., 129, 10229

19 wWw.strw. leidenuniv.nl/ ewine/photo 
Benedikt, J., Eijkman, D., Vandamme, W., Agarwal, S., \& van de Sanden, M. 2005, Chem. Phys. Lett., 402, 37

Bénilan, Y., Bruston, P., Raulin, F., Cossart-Magos, C., \& Guillemin, J.-C. 1994, J. Geophys. Res. - Planet., 99, 17069

Bergin, E., Calvet, N., D’Alessio, P., \& Herczeg, G. J. 2003, ApJ, 591, L159

Berkowitz, J. 1962, J. Chem. Phys., 36, 2533

Berkowitz, J. 2008, Int. J. Mass Spectr., 271, 8

Berkowitz, J., \& Cho, H. 1989, J. Chem. Phys., 90,

Bethell, T., \& Bergin, E. 2009, Science, 326, 1675

Bethell, T. J., \& Bergin, E. A. 2011, ApJ, 739, 78

Biehl, H., Schönnenbeck, G., Stuhl, F., \& Staemmler, V. 1994, J. Chem. Phys., 101,3819

Birnstiel, T., Dullemond, C. P., \& Brauer, F. 2010, A\&A, 513, A79

Black, J. H., \& Dalgarno, A. 1977, ApJS, 34, 405

Black, J. H., \& van Dishoeck, E. F. 1987, ApJ, 322, 412

Black, J. H., Weisheit, J. C., \& Laviana, E. 1972, ApJ, 177, 567

Blackie, D., Blackwell-Whitehead, R., Stark, G., et al. 2011, J. Geophys. Res., 116, E12099

Blitz, M. A., \& Seakins, P. W. 2012, Chem. Soc. Rev., 41, 6318

Brint, P., Connerade, J.-P., Mayhew, C., \& Sommer, K. 1985, J. Chem. Soc Farad. T. 2, 81, 1643

Brion, C., Dyck, A., \& Cooper, G. 2005, J. Electron Spectr. Relat. Phenom., 144, 127

Brites, V., Hammoutène, D., \& Hochlaf, M. 2008, J. Phys. B, 41, 045101

Bruna, P. J., \& Hirsch, G. 1987, Mol. Phys., 61, 1359

Bruna, P., Kammer, W., \& Vasudevan, K. 1975, Chem. Phys., 9, 91

Bruna, P. J., Buenker, R. J., \& Peyerimhoff, S. D. 1976, J. Mol. Spectr., 32, 217

Bruna, P. J., Hirsch, G., Peyerimhoff, S. D., \& Buenker, R. J. 1981, Mol. Phys., 42, 875

Brzozowski, J., Elander, N., \& Erman, P. 1974, Phys. Scr., 9, 99

Brzozowski, J., Erman, P., \& Lyyra, M. 1976, Phys. Scr., 14, 290

Burton, G. R., Chan, W. F., Cooper, G., \& Brion, C. 1992, Chem. Phys., 167, 349

Burton, G. R., Chan, W. F., Cooper, G., et al. 1994, Can. J. Chem., 72, 529

Cacciani, P., Brandi, F., Velchev, I., et al. 2001, Eur. J. Phys. D, 15, 47

Calvert, J., \& Pitts, J. 1966, in Photochemistry (Wiley)

Cameron, M., Sivakumaran, V., Dillon, T. J., \& Crowley, J. N. 2002, Phys. Chem. Chem. Phys., 4, 3628

Cantu, A. M., \& Parkinson, W. H. 1988, Phys. Scr., 37, 336

Carmona-Novillo, E., Moccia, R., \& Spizzo, P. 1996, Chem. Phys., 210, 435

Carnovale, F., White, M., \& Brion, C. 1981, J. Electron Spectr. Relat. Phenom., 24,63

Carrington, A., \& Kennedy, R. A. 1984, J. Chem. Phys., 81, 91

Carroll, P. K., \& Collins, C. P. 1969, Can. J. Phys., 47, 563

Carter, V. L., Hudson, R. D., \& Breig, E. L. 1971, Phys. Rev. A, 4, 821

Caselli, P., \& Ceccarelli, C. 2012, A\&ARv, 20, 56

Cecchi-Pestellini, C., \& Aiello, S. 1992, MNRAS, 258, 125

Chan, W., Cooper, G., \& Brion, C. 1992, Chem. Phys., 168, 375

Chan, W., Cooper, G., \& Brion, C. 1993a, Chem. Phys., 170, 123

Chan, W., Cooper, G., \& Brion, C. 1993b, Chem. Phys., 178, 387

Chan, W. F., Cooper, G., \& Brion, C. E. 1993c, Chem. Phys., 170, 111

Chan, W. F., Cooper, G., Sodhi, R., \& Brion, C. E. 1993d, Chem. Phys., 170, 81

Chaparro Molano, G., \& Kamp, I. 2012, A\&A, 537, A138

Chen, F., \& Wu, C. 2004, J. Quant. Spectr. Rad. Transf., 85, 195

Cheng, B.-M., Chew, E. P., Hung, W.-C., Eberhard, J., \& Lee, Y.-P. 1998, J. Synchrotron Radiat., 5, 1041

Cheng, B.-M., Bahou, M., Lee, Y.-P., \& Lee, L. C. 2002, J. Geophys. Res., 107 A8

Cheng, B.-M., Lu, H.-C., Chen, H.-K., et al. 2006, ApJ, 647, 1535

Cheng, B.-M., Chen, H.-F., Lu, H.-C., et al. 2011, ApJS, 196, 1

Choi, M., Tatematsu, K., \& Kang, M. 2010, ApJ, 723, L34

Chupka, W. A., \& Berkowitz, J. 1967, J. Chem. Phys., 47, 2921

Clauberg, H., Minsek, D. W., \& Chen, P. 1992, J. Am. Chem. Soc., 114, 99

Cleeves, L. I., Adams, F. C., \& Bergin, E. A. 2013, ApJ, 772, 5

Cleeves, L. I., Bergin, E. A., Qi, C., Adams, F. C., \& Öberg, K. I. 2015, ApJ, 799, 204

Clement, S. G., Ashfold, M. N. R., Western, C. M., Johnson, R. D., \& Hudgens, J. W. 1992, J. Chem. Phys., 97, 7064

Condon, E. U. 1928, Phys. Rev., 32, 858

Connors, R. E., Roebber, J. L., \& Weiss, K. 1974, J. Chem. Phys., 60, 5011

Cook, G. R., \& Ogawa, M. 1969, J. Chem. Phys., 51, 2419

Cool, T. A., Wang, J., Nakajima, K., Taatjes, C. A., \& Mcllroy, A. 2005, Int. J. Mass Spectrom., 247, 18

Cooper, G., Burton, G. R., \& Brion, C. 1995, J. Electron Spectr. Relat. Phenom., 73, 139

Cooper, G., Anderson, J. E., \& Brion, C. 1996, Chem. Phys., 209, 61

Coppens, P., \& Drowart, J. 1995, Chem. Phys. Lett., 243, 108

Cosby, P., \& Helm, H. 1988, Chem. Phys. Lett., 152, 71

Cravens, T. E., \& Dalgarno, A. 1978, ApJ, 219, 750
Crovisier, J., Leech, K., Bockelee-Morvan, D., et al. 1997, Science, 275, 1904 Curdt, W., Brekke, P., Feldman, U., et al. 2001, A\&A, 375, 591

D'Alessio, P., Calvet, N., \& Hartmann, L. 2001, ApJ, 553, 321

Dalgarno, A. 2006, Pro. Nat. Acad. Sci., 103, 12269

Dalgarno, A., Herzberg, G., \& Stephens, T. L. 1970, ApJ, 162, L49

Daviel, S., Iida, Y., Carnovale, F., \& Brion, C. 1984, Chem. Phys., 83, 391

Davis, D. D., \& Okabe, H. 1968, J. Chem. Phys., 49, 5526

Day, R. L., Suto, M., \& Lee, L. C. 1982, J. Phys. B, 15, 4403

de Almeida, A. A., \& Singh, P. D. 1986, Earth Moon and Planets, 36, 117

de Beer, E., Born, M., de Lange, C., \& Westwood, N. 1991, Chem. Phys. Lett., 186,40

de Oliveira, N., Roudjane, M., Joyeux, D., et al. 2011, Nature Photonics, 5, 149

Dehmer, P. M., \& Chupka, W. A. 1976, J. Chem. Phys., 65, 2243

Dehmer, P. M., Berkowitz, J., \& Chupka, W. A. 1974, J. Chem. Phys., 60, 2676

Dent, W. R. F., Wyatt, M. C., Roberge, A., et al. 2014, Science, 343, 1490

Dibeler, V. H. 1967, J. Chem. Phys., 47, 2191

Dibeler, V. H., \& Liston, S. K. 1967, J. Chem. Phys., 47, 4548

Dibeler, V. H., \& Walker, J. A. 1967, J. Opt. Soc. Amer., 57, 1007

Dickenson, G. D., \& Ubachs, W. 2014, Mol. Phys., 112, 1089

Dodson, L. G., Shen, L., Savee, J. D., et al. 2015, J. Phys. Chem. A, 119, 1279

Donovan, R. J., Husain, D., \& Stevenson, C. D. 1970, Trans. Faraday Soc., 66, 1 Draine, B. T. 1978, ApJS, 36, 595

Draine, B. 2003a, ARA\&A, 41, 241

Draine, B. T. 2003b, ApJ, 598, 1017

Draine, B. T. 2003c, ApJ, 598, 1026

Draine, B. T., \& Lee, H. M. 1984, ApJ, 285, 89

Draine, B. T., Dale, D. A., Bendo, G., et al. 2007, ApJ, 663, 866

Dressler, K. 1969, Can. J. Phys., 47, 547

Dunn, G. H. 1968, Phys. Rev., 172, 1

Eddington, A. S. 1928, MNRAS, 88, 352

Eden, S., Limão-Vieira, P., Kendall, P., et al. 2003, Eur. J. Phys. D, 26, 201

Edvardsson, D., Baltzer, P., Karlsson, L., et al. 1999, J. Phys. B, 32, 2583

Eidelsberg, M., \& Rostas, F. 1990, A\&A, 235, 472

Eidelsberg, M., Benayoun, J., Viala, Y., et al. 1992, A\&A, 265, 839

Eidelsberg, M., Sheffer, Y., Federman, S. R., et al. 2006, ApJ, 647, 1543

Eidelsberg, M., Lemaire, J. L., Federman, S. R., et al. 2012, A\&A, 543, A69

Eidelsberg, M., Lemaire, J. L., Federman, S. R., et al. 2014, A\&A, 566, A96

el-Qadi, W. H., \& Stancil, P. C. 2013, ApJ, 779, 97

Endo, Y., Danielache, S. O., Ueno, Y., et al. 2015, J. Geophys. Res.: Atmos., 120, 2546

Engel, V., \& Schinke, R. 1988, J. Chem. Phys., 88, 6831

Engin, S., Sisourat, N., \& Carniato, S. 2012, J. Chem. Phys., 137

Ernest, C. T., Bauer, D., \& Hynes, A. J. 2012, J. Phys. Chem. A, 116, 5910

Federman, S. R., Fritts, M., Cheng, S., et al. 2001, ApJS, 134, 133

Feller, D., \& Sordo, J. A. 2000, J. Chem. Phys., 113, 485

Feng, R., \& Brion, C. 2002, Chem. Phys., 282, 419

Feng, R., Cooper, G., \& Brion, C. 1999a, Chem. Phys., 244, 127

Feng, R., Cooper, G., \& Brion, C. 1999b, Chem. Phys., 249, 223

Feng, R., Cooper, G., Burton, G. R., Brion, C., \& Avaldi, L. 1999c, Chem. Phys., 240, 371

Feng, R., Cooper, G., \& Brion, C. E. 2000a, Chem. Phys., 252, 359

Feng, R., Cooper, G., Sakai, Y., \& Brion, C. 2000b, Chem. Phys., 255, 353

Ferland, G. J. 2003, ARA\&A, 41, 517

Ferradaz, T., Bénilan, Y., Fray, N., et al. 2009, Planet. Space Sci., 57, 10

Field, G. B., Somerville, W. B., \& Dressler, K. 1966, ARA\&A, 4, 207

Fillion, J.-H., Dulieu, F., Baouche, S., et al. 2003, J. Phys. B, 36, 2767

Fillion, J.-H., Ruiz, J., Yang, X.-F., et al. 2004, J. Chem. Phys., 120, 6531

Fischer, I., Lochschmidt, A., Strobel, A., et al. 1993, Chem. Phys. Lett., 202, 542

France, K., Schindhelm, E., Bergin, E. A., Roueff, E., \& Abgrall, H. 2014, ApJ, 784, 127

Frederix, P. W. J. M., Yang, C.-H., Groenenboom, G. C., et al. 2009, J. Phys. Chem. A, 113, 14995

Frerking, M. A., Keene, J., Blake, G. A., \& Phillips, T. G. 1989, ApJ, 344, 311

Fridh, C., \& Asbrink, L. 1975, J. Electron Spectr. Relat. Phenom., 7, 119

Frohlich, H., \& Glass-Maujean, M. 1990, Phys. Rev. A, 42, 1396

Gallagher, J. W., Brion, C. E., Samson, J. A. R., \& Langhoff, P. W. 1988, J. Phys. Chem. Ref. Data, 17, 9

Gans, B., Mendes, L. A. V., Boyé-Péronne, S., et al. 2010, J. Phys. Chem. A, 114,3237

Gans, B., Boye-Peronne, S., Broquier, M., et al. 2011, Phys. Chem. Chem. Phys., 13,8140

Gao, Y., \& Gao, T. 2014, Spectrochim. Acta. A, 118, 308

Gao, H., Song, Y., Chang, Y.-C., et al. 2013, J. Phys. Chem. A, 117, 6185

Garcia, G. A., Tang, X., Gil, J.-F., et al. 2015, J. Chem. Phys., 142

Gardner, E. P., \& McNesby, J. R. 1982, J. Phys. Chem., 86, 2646

Gibson, S. T., \& Lewis, B. R. 1996, J. Electron Spectr. Relat. Phenom., 80, 9

Gibson, S. T., Greene, J. P., \& Berkowitz, J. 1985, J. Chem. Phys., 83, 4319 
A. N. Heays et al.: Photodissociation and photoionisation of atoms and molecules of astrophysical interest

Gibson, S. T., Greene, J. P., Ruscic, B., \& Berkowitz, J. 1986, J. Phys. B, 19, 2825

Gingell, J., Mason, N., Zhao, H., Walker, I., \& Siggel, M. 1997, Chem. Phys., 220,191

Ginter, D. S., \& Ginter, M. L. 1981, J. Mol. Spectr., 90, 177

Glass-Maujean, M., Jungen, C., Reichardt, G., et al. 2010, Phys. Rev. A, 82, 062511

Glass-Maujean, M., Jungen, C., Schmoranzer, H., et al. 2013a, J. Mol. Spectr., 293, 11

Glass-Maujean, M., Jungen, C., Schmoranzer, H., et al. 2013b, J. Mol. Spectr., 293, 19

Glass-Maujean, M., Jungen, C., Spielfiedel, A., et al. 2013c, J. Mol. Spectr., 293,

Glassgold, A. E. 1996, ARA\&A, 34, 241

Glover, S. C. O., \& Clark, P. C. 2012, MNRAS, 421, 116

Grebenshchikov, S. Y., Qu, Z.-W., Zhu, H., \& Schinke, R. 2007, Phys. Chem. Chem. Phys., 9, 2044

Gredel, R., \& Dalgarno, A. 1995, ApJ, 446, 852

Gredel, R., Lepp, S., \& Dalgarno, A. 1987, ApJ, 323, L137

Gredel, R., Lepp, S., Dalgarno, A., \& Herbst, E. 1989, ApJ, 347, 289

Green, D. S., Bickel, G. A., \& Wallace, S. C. 1991, J. Mol. Spectr., 150, 303

Grenier, I. A., Black, J. H., \& Strong, A. W. 2015, ARA\&A, 53, 199

Grosch, H., Fateev, A., \& Clausen, S. 2015, J. Quant. Spectr. Rad.. Transf., 154

Guberman, S. L. 1977, J. Chem. Phys., 67, 1125

Gustafsson, O., Larsson, M., \& Sigray, P. 1988, Z. Phys. D, 7, 373

Guyon, P. M., Chupka, W. A., \& Berkowitz, J. 1976, J. Chem. Phys., 64, 1419

Guzman, V. V., Pety, J., Gratier, P., et al. 2014, Faraday Discuss., 168, 103

Habing, H. J. 1968, Bull. Astron. I. Neth., 19, 421

Halpern, J., Miller, G., Okabe, H., \& Nottingham, W. 1988, J. Photoch. Photobio. A, 42,63

Hansen, J. E., Ziegenbein, B., Lincke, R., \& Kelly, H. P. 1977, J. Phys. B, 10, 37

Harrison, A. J., Cederholm, B. J., \& Terwilliger, M. A. 1959, J. Chem. Phys., 30 355

Harrison, H., Schoen, R., \& Cairns, R. 1969, J. Chem. Phys., 50, 3930

Hart, D. J., \& Hepburn, J. W. 1987, J. Chem. Phys., 86, 1733

Hartquist, T. W., Black, J. H., \& Dalgarno, A. 1978, MNRAS, 185, 643

Hasegawa, T. I., \& Kwok, S. 2003, ApJ, 585, 475

Haser, L. 1957, Bull. Soc. R. Sci. Liege, 43, 740

Hatano, Y. 1999, Phys. Rep., 313, 109

Heays, A. N. 2011, Ph.D. Thesis, The Australian National University

Heays, A. N., Dickenson, G. D., Salumbides, E. J., et al. 2011, J. Chem. Phys., 135,244301

Heays, A. N., Lewis, B. R., Gibson, S. T., et al. 2012, Phys. Rev. A, 85, 012705

Heays, A. N., Ajello, J. M., Aguilar, A., Lewis, B. R., \& Gibson, S. T. 2014a ApJS, 211, 28

Heays, A. N., Visser, R., Gredel, R., et al. 2014b, A\&A, 562, A61

Heazlewood, B. R., Rowling, S. J., Maccarone, A. T., Jordan, M. J. T., \& Kable, S. H. 2009, J. Chem. Phys., 130

Herzberg, G. 1966, Molecular spectra and molecular structure, Vol. 3, Electronic spectra of polyatomic molecules (New York: van Nostrand)

Herzberg, G. 1989, in Molecular Spectra and Molecular Structure I: Spectra of Diatomic Molecules, 2nd edn. (Krieger Publishing Company)

Herzberg, G., \& Innes, K. K. 1957, Can. J. Phys., 35, 842

Herzberg, G., \& Shoosmith, J. 1956, Can. J. Phys., 34, 523

Hezareh, T., Houde, M., McCoey, C., Vastel, C., \& Peng, R. 2008, ApJ, 684, 1221

Hikida, T., \& Mori, Y. 1978, J. Chem. Phys., 69, 346

Ho, P. T. P., \& Townes, C. H. 1983, ARA\&A, 21, 239

Holland, D. M. P., \& Shaw, D. A. 2014, J. Phys. B, 47, 025101

Holland, D., Shaw, D., McSweeney, S., et al. 1993, Chem. Phys., 173, 315

Holland, D., Shaw, D., \& Hayes, M. 1995, Chem. Phys., 201, 299

Hollenbach, D., \& Tielens, A. 1999, Rev. Mod. Phys., 71, 173

Hollenbach, D. J., Takahashi, T., \& Tielens, A. G. G. M. 1991, ApJ, 377, 192

Hollenstein, U., Reinhold, E., de Lange, C., \& Ubachs, W. 2006, J. Phys. B, 39, L195

Holt, R. B., McLane, C. K., \& Oldenberg, O. 1948, J. Chem. Phys., 16, 225

Honjou, N., Tanaka, K., Ohno, K., \& Taketa, H. 1978, Mol. Phys., 35, 1569

Hopkins, W. S., Loock, H.-P., Cronin, B., et al. 2007, J. Chem. Phys., 127, 064301

Hotop, H., Hübler, G., \& Kaufhold, L. 1975, Int. J. Mass Spectrom., 17, 163

Hsu, Y.-C., Smith, M. A., \& Wallace, S. C. 1984, Chem. Phys. Lett., 111, 219

Hsu, C., Baldwin, D. P., Liao, C., \& Ng, C. Y. 1994, J. Chem. Phys., 100, 8047

Hu, Z., Wang, Z., Kong, X., et al. 2002, Acta. Phys. Sin., 51, 235

Huang, Y., Barts, S. A., \& Halpern, J. B. 1992, J. Phys. Chem., 96, 425

Huber, K. P., \& Herzberg, G. 1979, in Molecular spectra and molecular structure IV: Constants of diatomic molecules (New York: Van Nostrand)
Hubin-Franskin, M.-J., Delwiche, J., Giuliani, A., et al. 2002, J. Chem. Phys., 116, 9261

Hudson, R. D., \& Carter, V. L. 1968, J. Opt. Soc. Amer., 58, 227

Huebner, W., \& Mukherjee, J. 2015, Planet. Space Sci., 106, 11

Huebner, W., Keady, J., \& Lyon, S. 1992, Astrophys. Space. Sci, 195, 1

Huestis, D. L., \& Berkowitz, J. 2010, in BAAS, 42, AAS/Division for Planetary Sciences Meeting Abstracts \#42, 972

Hurzeler, H., Inghram, M. G., \& Morrison, J. D. 1958, J. Chem. Phys., 28, 76

Iida, Y., Carnovale, F., Daviel, S., \& Brion, C. 1986, Chem. Phys., 105, 211

Indriolo, N., \& McCall, B. J. 2012, ApJ, 745, 91

Indriolo, N., Neufeld, D. A., Gerin, M., et al. 2015, ApJ, 800, 40

Inokuti, M. 1971, Rev. Mod. Phys., 43, 297

Jansen, D. J., van Dishoeck, E. F., Black, J. H., Spaans, M., \& Sosin, C. 1995, A\&A, 302, 223

Jansen, D. J., van Dishoeck, E. F., Keene, J., Boreiko, R. T., \& Betz, A. L. 1996, A\&A, 309, 899

Jochims, H. W., Ruhl, E., Baumgärtel, H., Tobita, S., \& Leach, S. 1994, ApJ, 420,307

Johansen, A., Oishi, J. S., Mac Low, M.-M., Klahr, H., \& Henning, T. 2007, Nature, 448, 1022

Johns, J. W. C., \& Ramsay, D. A. 1961, Can. J. Phys., 39, 210

Jonas, D. M., Zhao, X., Yamanouchi, K., et al. 1990, J. Chem. Phys., 92, 3988

Jonin, C., Liu, X., Ajello, J., James, G., \& Abgrall, H. 2000, ApJS, 129, 247

Jonkheid, B., Kamp, I., Augereau, J.-C., \& van Dishoeck, E. F. 2006, A\&A, 453, 163

Kameta, K., Machida, S., Kitajima, M., et al. 1996, J. Electron Spectr. Relat. Phenom., 79, 391

Kameta, K., Kouchi, N., Ukai, M., \& Hatano, Y. 2002, J. Electron Spectr. Relat. Phenom., 123, 225

Katagiri, H., Sako, T., Hishikawa, A., et al. 1997, J. Mol. Struct., 413, 589

Kato, H., \& Baba, M. 1995, Chem. Rev., 95, 2311

Kelly, R. 1987a, J. Phys. Chem. Ref. Data, 16, 1

Kelly, R. 1987b, J. Phys. Chem. Ref. Data, 16, 651

Kessler-Silacci, J., Augereau, J.-C., Dullemond, C. P., et al. 2006, ApJ, 639, 275

Khamaganov, V., Karunanandan, R., Rodriguez, A., \& Crowley, J. N. 2007, Phys. Chem. Chem. Phys., 9, 4098

Kim, S. G., Lee, Y. H., Nordlander, P., \& Tománek, D. 1997, Chem. Phys. Lett., 264, 345

Kinghorn, D. B., \& Adamowicz, L. 1997, J. Chem. Phys., 106, 4589

Kirby, K., \& Dalgarno, A. 1978, ApJ, 224, 444

Kirby, K. P., \& Goldfield, E. M. 1991, J. Chem. Phys., 94, 1271

Kirby, K., \& van Dishoeck, E. 1988, Adv. At. Mol. Phys., 25, 437

Kirby, K., Saxon, R. P., \& Liu, B. 1979, ApJ, 231, 637

Kirby, K., Roberge, W. G., Saxon, R. P., \& Liu, B. 1980, ApJ, 239, 855

Klynning, L., \& Pages, P. 1982, Phys. Scr., 25, 543

Knowles, D. J., \& Nicholson, A. J. C. 1974, J. Chem. Phys., 60, 1180

Koch, A. 1997, J. Phys. Chem. A, 101, 1460

Koch, A., van Dishoeck, E. F., \& van Hemert, M. C. 1995a, Ber. Bunsen-Ges. Phys. Chem., 99, 393

Koch, A., van Hemert, M. C., \& van Dishoeck, E. F. 1995b, J. Chem. Phys., 103, 7006

Kohl, J. L., \& Parkinson, W. H. 1973, ApJ, 184, 641

Koizumi, H., Hironaka, K., Shinsaka, K., et al. 1986, J. Chem. Phys., 85, 4276

Kovács, T., Blitz, M. A., \& Seakins, P. W. 2010, J. Phys. Chem. A, 114, 4735

Kramers, H. A., \& Ter Haar, D. 1946, Bull. Astron. I. Neth., 10, 137

Kramida, A. 2010, At. Dat. Nucl. Tabl., 96, 586

Krishnamurty, G., \& Narasimham, N. 1969, J. Mol. Spectr., 29, 410

Kroes, G.-J., van Hemert, M. C., Billing, G., \& Neuhauser, D. 1997, Chem. Phys. Lett., 271, 311

Kulander, K. C., \& Bottcher, C. 1978, Chem. Phys., 29, 141

Kulik, H. J., Steeves, A. H., \& Field, R. W. 2009, J. Mol. Spectr., 258, 6

Kutina, R. E., Edwards, A. K., Goodman, G. L., \& Berkowitz, J. 1982, J. Chem. Phys., 77, 5508

Lammer, H., Güdel, M., Kulikov, Y., et al. 2012, Earth Planets Space, 64, 179

Langhoff, P. 1985, in Molecular astrophysics (Springer)

Larsson, M. 1983, A\&A, 128, 291

Läuter, A., Lee, K., Jung, K., et al. 2002, Chem. Phys. Lett., 358, 314

Lavendy, H., Gandara, G., \& Robbe, J. 1984, J. Mol. Spectr., 106, 395

Lavendy, H., Robbe, J. M., \& Gandara, G. 1987, J. Phys. B, 20, 3067

Lavendy, H., Robbe, J. M., \& Flament, J. P. 1993, Chem. Phys. Lett., 205, 456

Le Page, V., Snow, T. P., \& Bierbaum, V. M. 2003, ApJ, 584, 316

Le Petit, F., Nehmé, C., Le Bourlot, J., \& Roueff, E. 2006, ApJS, 164, 506

Leach, S., Jochims, H.-W., \& Baumgärtel, H. 2005, Phys. Chem. Chem. Phys., 7, 900

Leach, S., Jochims, H.-W., \& Baumgärtel, H. 2010, J. Phys. Chem. A, 114, 4847

Leach, S., Garcia, G. A., Mahjoub, A., et al. 2014, J. Chem. Phys., 140

Lee, L. C. 1980, J. Chem. Phys., 72, 6414

Lee, L. C. 1984, ApJ, 282, 172 
Lee, L. C., \& Chiang, C. C. 1982, J. Chem. Phys., 76, 4462

Lee, S.-H. 2009, J. Chem. Phys., 131, 11138

Lee, L. C., Wang, X., \& Suto, M. 1987, J. Chem. Phys., 86, 4353

Lee, H.-H., Herbst, E., Pineau des Forets, G., Roueff, E., \& Le Bourlot, J. 1996, A\&A, 311, 690

Lee, S., Sun, H., Kim, B., \& Freed, K. F. 2001, J. Chem. Phys., 114, 5537

Leger, A., D'Hendecourt, L., Boissel, P., \& Desert, F. X. 1989, A\&A, 213, 351

Leitherer, C., Otálvaro, P. A. O., Bresolin, F., et al. 2010, ApJS, 189, 309

Leroy, A. K., Bolatto, A., Gordon, K., et al. 2011, ApJ, 737, 12

Levin, J., Hechtfischer, U., Knoll, L., et al. 2000, Hyperfine Interactions, 127, 267

Lewerenz, M., Bruna, P. J., Peyerimhoff, S. D., \& Buenker, R. J. 1983, Mol. Phys., 49, 1

Lewis, B. R., Gibson, S. T., Torop, L. W., \& McCoy, D. 1998, Geophys. Res. Lett., 25, 2457

Lewis, B. R., England, J. P., Gibson, S. T., Brunger, M. J., \& Allan, M. 2001, Phys. Rev. A, 63, 022707

Lewis, B. R., Gibson, S. T., England, J. P., Stark, G., \& West, J. B. 2002, J. Chem Phys., 116, 3286

Lewis, B. R., Gibson, S. T., Sprengers, J. P., et al. 2005, J. Chem. Phys., 123, 236101

Lewis, B. R., Baldwin, K. G. H., Sprengers, J. P., et al. 2008, J. Chem. Phys., 129,164305

Li, A., \& Draine, B. 2001, ApJ, 554, 778

Li, A., \& Lunine, J. I. 2003, ApJ, 590, 368

Li, X., Heays, A. N., Visser, R., et al. 2013, A\&A, 555, A14

Li, X., Millar, T. J., Walsh, C., Heays, A. N., \& van Dishoeck, E. F. 2014, A\&A 568, A111

Liang, M.-C., Cheng, B.-M., Lu, H.-C., et al. 2007, ApJ, 657, L117

Liao, C. L., \& Ng, C. Y. 1986, J. Chem. Phys., 84, 778

Limão Vieira, P., Ferreira da Silva, F., Almeida, D., et al. 2015, J. Chem. Phys., 142

Lin, C. L., Rohatgi, N. K., \& DeMore, W. B. 1978, Geophys. Res. Lett., 5, 113

Liseau, R., Larsson, B., Lunttila, T., et al. 2015, A\&A, 578, A131

Litorja, M., \& Ruscic, B. 1998, J. Electron Spectr. Relat. Phenom., 97, 131

Löffler, P., Lacombe, D., Ross, A., et al. 1996, Chem. Phys. Lett., 252, 304

Loison, J., Hébrard, E., Dobrijevic, M., et al. 2015, Icarus, 247, 218

Lombardi, G. G., Smith, P. L., \& Parkinson, W. H. 1978, Phys. Rev. A, 18, 2131

Lu, H.-C., Chen, H.-K., Chen, H.-F., Cheng, B.-M., \& Ogilvie, J. F. 2010, A\&A, 520, A19

Luque, J., \& Crosley, D. 2000, J. Chem. Phys., 112, 9411

Manatt, S. L., \& Lane, A. L. 1993, J. Quant. Spectr. Rad. Transf., 50, 267

Marinelli, F., \& Allouche, A. 1982, Theor. Chim. Acta, 62, 175

Marr, G. V., \& Austin, J. M. 1969, J. Phys. B, 2, 107

Mason, N. J., Gingell, J. M., Davies, J. A., et al. 1996, J. Phys. B, 29, 3075

Mathews, B., Krivov, A., Wyatt, M., Bryden, G., \& Eiroa, C. 2014, in Protostars and planets VI (University of Arizona Press)

Mathis, J. S., Rumpl, W., \& Nordsieck, K. H. 1977, ApJ, 217, 425

Mathis, J. S., Mezger, P. G., \& Panagia, N. 1983, A\&A, 128, 212

Matos, J. M. O., Malmqvist, P.-A., \& Roos, B. O. 1987, J. Chem. Phys., 86, 5032

Matsunaga, F. M., \& Watanabe, K. 1967, Sci. Light, 16, 31

Mayer, E., \& Grant, E. R. 1995, J. Chem. Phys., 103, 10513

McAdam, K., Veyret, B., \& Lesclaux, R. 1987, Chem. Phys. Lett., 133, 39

McElroy, D., Walsh, C., Markwick, A. J., et al. 2013, A\&A, 550, A36

McGuire, E. J. 1968, Phys. Rev., 175, 20

McLaughlin, B. M. 2001, in Spectroscopic Challenges of Photoionized Plasmas, eds. G. Ferland \& D. W. Savin, ASP Conf. Ser., 247

McMillan, E. C., Shen, G., McCann, J. F., McLaughlin, B. M., \& Stancil, P. C. 2016, J. Phys. B, 49, 084001

Mebel, A. M., Jackson, W. M., Chang, A. H. H., \& Lin, S. H. 1998, J. Am. Chem. Soc., 120, 5751

Meller, R., \& Moortgat, G. K. 2000, J. Geophys. Res. - Atmos., 105, 7089

Mendoza, C. 1996, Phys. Scr., 1996, 198

Mendoza, C., \& Zeippen, C. J. 1988, J. Phys. B, 21, 259

Mentall, J. E., Gentieu, E. P., Krauss, M., \& Neumann, D. 1971, J. Chem. Phys., 55,5471

Metzger, P. H., \& Cook, G. R. 1964, J. Chem. Phys., 41, 642

Michael, J., \& Noyes, W. 1963, J. Am. Chem. Soc., 85, 1228

Miyake, S., Gay, C. D., \& Stancil, P. C. 2011, ApJ, 735, 21

Mocellin, A., Wiesner, K., Burmeister, F., Björneholm, O., \& Naves de Brito, A 2001, J. Chem. Phys., 115, 5041

Molina, L. T., Lamb, J. J., \& Molina, M. J. 1981, Geophys. Res. Lett., 8, 1008

Monninger, G., Forderer, M., Gurtler, P., et al. 2002, J. Phys. Chem. A, 106, 5779

Mordaunt, D. H., Ashfold, M. N. R., \& Dixon, R. N. 1994, J. Chem. Phys., 100, 7360

Morgan, R. A., Puyuelo, P., Howe, J. D., et al. 1995, J. Chem. Soc. Farad. Trans. 91,2715
Morley, G. P., Lambert, I. R., Ashfold, M. N. R., Rosser, K. N., \& Western, C. M. 1992, J. Chem. Phys., 97, 3157

Mota, R., Parafita, R., Giuliani, A., et al. 2005, Chem. Phys. Lett., 416, 152

Mottram, J. C., Kristensen, L. E., van Dishoeck, E. F., et al. 2014, A\&A, 572, A21

Muto, T., Grady, C. A., Hashimoto, J., et al. 2012, ApJ, 748, L22

Nahar, S. N., \& Pradhan, A. K. 1993, J. Phys. B, 26, 1109

Nahar, S. N., \& Pradhan, A. K. 1997, ApJS, 111, 339

Nee, J. B., \& Lee, L. C. 1986, J. Chem. Phys., 84, 5303

Nee, J., Suto, M., \& Lee, L. 1985, Chem. Phys., 98, 147

Nee, J. B., Suto, M., \& Lee, L. C. 1986, J. Chem. Phys., 85, 719

Neufeld, D. A. 1991, ApJ, 370, L85

Neufeld, D. A., \& Dalgarno, A. 1989, ApJ, 344, 251

Neufeld, D. A., \& Wolfire, M. G. 2009, ApJ, 706, 1594

Nicolas, C., Shu, J., Peterka, D., et al. 2006, J. Am. Chem. Soc., 128, 220

Niu, M. L., Salumbides, E. J., Dickenson, G. D., Eikema, K. S. E., \& Ubachs, W. 2014, J. Mol. Spectr., 300, 44

Nomura, H., \& Millar, T. J. 2005, A\&A, 438, 923

Norwood, K., \& Ng, C. 1989, Chem. Phys. Lett., 156, 145

Nuth, J. A., \& Glicker, S. 1982, J. Quant. Spectr. Rad. Transf. 28, 223

Ogawa, M., \& Cook, G. R. 1958, J. Chem. Phys., 28, 173

Ogawa, S., \& Ogawa, M. 1975, Can. J. Phys., 53, 1845

Oka, T. 2013, Chem. Rev., 113, 8738

Okabe, H. 1972, J. Chem. Phys., 56, 4381

Okabe, H. 1978, in Photochemistry of small molecules (New York: WileyInterscience)

Okabe, H. 1983, Can. J. Phys., 61, 850

Ortiz, J. V. 1993, J. Chem. Phys., 99, 6716

Osterbrock, D. E. 1979, ApJ, 84, 901

Padovani, M., Galli, D., \& Glassgold, A. E. 2009, A\&A, 501, 619

Page, R. H., Larkin, R. J., Shen, Y. R., \& Lee, Y. T. 1988, J. Chem. Phys., 88, 2249

Palenius, H., Kohl, J., \& Parkinson, W. 1976, Phys. Rev. A, 13, 1805

Parkinson, W., \& Yoshino, K. 2003, Chem. Phys., 294, 31

Parravano, A., Hollenbach, D. J., \& McKee, C. F. 2003, ApJ, 584, 797

Patel-Misra, D., Parlant, G., Sauder, D. G., Yarkony, D. R., \& Dagdigian, P. J. 1991, J. Chem. Phys., 94, 1913

Peebles, L. R., \& Marshall, P. 2002, J. Chem. Phys., 117, 3132

Petrank, A., Iraqi, M., Dotan, I., \& Lifshitz, C. 1992, Int. J. Mass Spectrom., 117, 223

Philip, J., Sprengers, J., Pielage, T., et al. 2004, Can. J. Chem., 82, 713

Phillips, L. F. 1981, J. Phys. Chem., 85, 3994

Pinilla, P., de Juan Ovelar, M., Ataiee, S., et al. 2015, A\&A, 573, A9

Plusquellic, D. F., Votava, O., \& Nesbitt, D. J. 1998, J. Chem. Phys., 109, 6631

Pope, F. D., Smith, C. A., Ashfold, M. N. R., \& Orr-Ewing, A. J. 2005, Phys. Chem. Chem. Phys., 7, 79

Pouilly, B., Robbe, J. M., Schamps, J., \& Roueff, E. 1983, J. Phys. B, 16, 437

Pradhan, A. D., Kirby, K. P., \& Dalgarno, A. 1991, J. Chem. Phys., 95, 9009

Prasad, S. S., \& Tarafdar, S. P. 1983, ApJ, 267, 603

Reddy, R., Ahammed, Y., Gopal, K., Azeem, P., \& Rao, T. 2000, J. Quant. Spectr. Rad. Transf., 66, 501

Reed, B., Lam, C.-S., Chang, Y.-C., et al. 2009, ApJ, 693, 940

Reilman, R. F., \& Manson, S. T. 1979, ApJS, 40, 815

Resende, S. M., \& Ornellas, F. R. 2001, J. Chem. Phys., 115, 2178

Rimmer, P. B., Herbst, E., Morata, O., \& Roueff, E. 2012, A\&A, 537, A7

Roberge, W. G., Jones, D., Lepp, S., \& Dalgarno, A. 1991, ApJS, 77, 287

Röllig, M., Szczerba, R., Ossenkopf, V., \& Glück, C. 2013, A\&A, 549, A85

Rollins, R. P., \& Rawlings, J. M. C. 2012, MNRAS, 427, 2328

Roman-Duval, J., Gordon, K. D., Meixner, M., et al. 2014, ApJ, 797, 86

Romanzin, C., Gazeau, M.-C., Bénilan, Y., et al. 2005, Adv. Space Res., 36, 258

Roncin, J. Y., Launay, F., \& Yoshino, K. 1989, J. Mol. Spectr., 134, 390

Roueff, E., Alekseyev, A. B., \& Le Bourlot, J. 2014, A\&A, 566, A30

Ruscic, B., \& Berkowitz, J. 1983, Phys. Rev. Lett., 50, 675

Salahub, D., \& Sandorfy, C. 1971, Chem. Phys. Lett., 8, 71

Samson, J. A. R., \& Angel, G. C. 1990, Phys. Rev. A, 42, 1307

Samson, J. A. R., \& Haddad, G. N. 1994, J. Opt. Soc. Am. B, 11, 277

Samson, J. A. R., Haddad, G. N., \& Kilcoyne, L. D. 1987, J. Chem. Phys., 87, 6416

Sandner, W., Gallagher, T. F., Safinya, K. A., \& Gounand, F. 1981, Phys. Rev. A, 23,2732

Sansonetti, J. E. 2006, J. Phys. Chem. Ref. Data, 35, 301

Savage, B. D., Bohlin, R. C., Drake, J. F., \& Budich, W. 1977, ApJ, 216, 291

Sawyer, D. T. 1989, J. Phys. Chem., 93, 7977

Saxon, R. P., \& Liu, B. 1986, J. Chem. Phys., 85, 2099

Saxon, R. P., Lengsfield, B. H., \& Liu, B. 1983, J. Chem. Phys., 78, 312

Schindhelm, E., France, K., Herczeg, G. J., et al. 2012, ApJ, 756, L23

Schulenburg, A. M., Alcaraz, C., Grassi, G., \& Merkt, F. 2006, J. Chem. Phys., 125, 104310 
Schwell, M., Jochims, H.-W., Baumgärtel, H., \& Leach, S. 2008, Chem. Phys., 344,164

Seaton, M. J. 1951, MNRAS, 111, 368

Seaton, M. J. 1985, J. Phys. B, 18, 2111

Seki, K., \& Okabe, H. 1993, J. Phys. Chem., 97, 5284

Seki, K., He, M., Liu, R., \& Okabe, H. 1996, J. Phys. Chem., 100, 5349

Serdyuchenko, A., Gorshelev, V., Weber, M., Chehade, W., \& Burrows, J. P. 2014, Atm. Meas. Tech., 7, 625

Shafer, N., Satyapal, S., \& Bersohn, R. 1989, J. Chem. Phys., 90, 6807

Shaw, D. A., Holland, D. M. P., MacDonald, M. A., et al. 1992, Chem. Phys., 166,379

Shaw, D., Holland, D., Hayes, M., et al. 1995, Chem. Phys., 198, 381

Shi, D., Li, W., Sun, J., \& Zhu, Z. 2011, J. Quant. Spectr. Rad. Transf., 112, 2335

Shiell, R. C., Hu, X. K., Hu, Q. J., \& Hepburn, J. W. 2000, J. Phys. Chem. A 104,4339

Shinnaka, Y., Kawakita, H., Kobayashi, H., et al. 2011, ApJ, 729, 81

Silver, J. A., Worsnop, D. R., Freedman, A., \& Kolb, C. E. 1986, J. Chem. Phys., 84,4378

Slanger, T. G., \& Black, G. 1982, J. Chem. Phys., 77, 2432

Smith, W., \& Liszt, H. 1971, J. Quant. Spectr. Rad. Transf., 11, 45

Smith, P. L., Yoshino, K., Griesinger, H. E., \& Black, J. H. 1981, ApJ, 250, 166

Sohl, J. E., Zhu, Y., \& Knight, R. D. 1990, J. Opt. Soc. Amer. B, 7, 9

Song, Y., Gao, H., Chang, Y. C., et al. 2014, Phys. Chem. Chem. Phys., 16, 563

Sorensen, S., Karawajczyk, A., Strömholm, C., \& Kirm, M. 1995, Chem. Phys. Lett., 232, 554

Spitzer, L., Drake, J. F., Jenkins, E. B., et al. 1973, ApJ, 181, L116

Sprengers, J. P., Ubachs, W., Baldwin, K. G. H., Lewis, B. R., \& Tchang-Brillet, W.-Ü. L. 2003, J. Chem. Phys., 119, 3160

Stahel, D., Leoni, M., \& Dressler, K. 1983, J. Chem. Phys., 79, 2541

Stark, G., Yoshino, K., \& Smith, P. 1987, J. Mol. Spectr., 124, 420

Stark, G., Lewis, B. R., Gibson, S. T., \& England, J. P. 1999, ApJ, 520, 732

Stark, G., Lewis, B. R., Heays, A. N., et al. 2008, J. Chem. Phys., 128, 114302

Stecher, T. P., \& Williams, D. A. 1967, ApJ, 149, L29

Stephens, T., \& Dalgarno, A. 1972, J. Quant. Spectr. Rad. Transf., 12, 569

Sternberg, A., \& Dalgarno, A. 1995, ApJS, 99, 565

Sternberg, A., Dalgarno, A., \& Lepp, S. 1987, ApJ, 320, 676

Sternberg, A., Petit, F. L., Roueff, E., \& Bourlot, J. L. 2014, ApJ, 790, 10

Stief, L. J., Payne, W. A., \& Klemm, R. B. 1975, J. Chem. Phys., 62, 4000

Suemitsu, H., \& Samson, J. A. R. 1983, Phys. Rev. A, 28, 2752

Sunanda, K., Shastri, A., Das, A. K., \& Sekhar, B. R. 2015, J. Quant. Spectr. Rad. Transf., 151, 76

Suto, M., \& Lee, L. 1983, Chem. Phys. Lett., 98, 152

Suto, M., Wang, X., \& Lee, L. C. 1986, J. Chem. Phys., 85, 4228

Szajna, W., \& Zachwieja, M. 2010, J. Mol. Spectr., 260, 130

Szajna, W., Zachwieja, M., Hakalla, R., \& Kepa, R. 2011, Acta Phys. Pol. A, 120,417

Taatjes, C. A., Klippenstein, S. J., Hansen, N., et al. 2005, Phys. Chem. Chem. Phys., 7, 806

Talbi, D., \& Saxon, R. P. 1988, J. Chem. Phys., 89, 2235

Tarroni, R., Palmieri, P., Mitrushenkov, A., Tosi, P., \& Bassi, D. 1997, J. Chem. Phys., 106, 10265

Tayal, S. S. 2004, J. Phys. B, 37, 3593

Tchang-Brillet, W.-Ü. L., Julienne, P., Robbe, J., Letzelter, C., \& Rostas, F. 1992, J. Chem. Phys., 96, 6735

Testi, L., Birnstiel, T., Ricci, L., et al. 2014, in Protostars \& Planets VI (Tucson: Univ. Arizona Press)

Teyssier, D., Fossé, D., Gerin, M., et al. 2004, A\&A, 417, 135

Theodorakopoulos, G., \& Petsalakis, I. 1991, J. Mol. Struct., 76, 205

Tielens, A. G. G. M. 2013, Rev. Mod. Phys., 85, 1021

Tielens, A. G. G. M., \& Hollenbach, D. 1985, ApJ, 291, 747

Tokue, I., Hiraya, A., \& Shobatake, K. 1987, Chem. Phys., 116, 449

Tondello, G. 1975, Mem. Soc. Astron. Ital., 46, 113

Tsukagoshi, T., Momose, M., Saito, M., et al. 2015, ApJ, 802, L7

Ubachs, W. 2005, J. Electron Spectr. Relat. Phenom., 144, 97

Ubachs, W., Eikema, K. S. E., Levelt, P. F., et al. 1994, ApJ, 427, L55

Ung, A.-M. 1974, Chem. Phys. Lett., 28, 603

Uzer, T., \& Dalgarno, A. 1978, Chem. Phys., 32, 301

Vaghjiani, G. L. 1993, J. Chem. Phys., 99, 5936

Valenti, J. A., Johns-Krull, C. M., \& Linsky, J. L. 2000, ApJS, 129, 399

van der Marel, N., van Dishoeck, E. F., Bruderer, S., et al. 2013, Science, 340, 1199

van der Tak, F. F. S., \& van Dishoeck, E. F. 2000, A\&A, 358, L79

van Dishoeck, E. F. 1987, J. Chem. Phys., 86, 196

van Dishoeck, E. F. 1988, in Rate coefficients in astrochemistry, eds. T. J. Millar,

\& D. A. Williams (Dordrecht: Kluwer Academic Publishers), 49

van Dishoeck, E. F. 2014, Faraday Discuss., 168, 9

van Dishoeck, E. F., \& Black, J. H. 1982, ApJ, 258, 533 van Dishoeck, E. F., \& Black, J. H. 1986, ApJS, 62, 109 van Dishoeck, E. F., \& Black, J. H. 1988, ApJ, 334, 771 van Dishoeck, E. F., \& Dalgarno, A. 1983, J. Chem. Phys., 79, 873 van Dishoeck, E. F., \& Dalgarno, A. 1984a, J. Chem. Phys., 277, 576 van Dishoeck, E. F., \& Dalgarno, A. 1984b, J. Chem. Phys., 277, 576 van Dishoeck, E. F., \& Visser, R. 2015, in Laboratory astrochemistry: From molecules through nanoparticles to grains (Weiheim: Wiley-VCH)

van Dishoeck, E. F., van der Hart, W. J., \& van Hemert, M. 1980, Chem. Phys., 50,45

van Dishoeck, E. F., van Hemert, M. C., \& Dalgarno, A. 1982, J. Chem. Phys., 77,3693

van Dishoeck, E. F., van Hemert, M. C., Allison, A. C., \& Dalgarno, A. 1984, J. Chem. Phys., 81, 5709

van Dishoeck, E. F., Beaerda, R. A., \& van Hemert, M. C. 1996, A\&A, 307, 645 van Dishoeck, E. F., Jonkheid, B., \& van Hemert, M. C. 2006, Faraday Discuss., 133,231

van Dishoeck, E. F., Herbst, E., \& Neufeld, D. A. 2013, Chem. Rev., 113, 9043 van Harrevelt, R., \& van Hemert, M. C. 2008, J. Phys. Chem. A, 112, 3002 van Hemert, M. C., \& van Dishoeck, E. F. 2008, Chem. Phys., 343, 292

van Mourik, T., Dunning, T. H., \& Peterson, K. A. 2000, J. Phys. Chem. A, 104, 2287

Vane, C. R., Bahati, E. M., Bannister, M. E., \& Thomas, R. D. 2007, Phys. Rev. A, 75, 052715

Vattulainen, J., Wallenius, L., Stenberg, J., Hernberg, R., \& Linna, V. 1997, App. Spectr., 51, 1311

Vaupré, S., Hily-Blant, P., Ceccarelli, C., et al. 2014, A\&A, 568, A50

Vega, M. V., Lavin, C., Velasco, A. M., \& Martin, I. 2010, Theor. Chem. Acc., 127,411

Venot, O., Fray, N., Bénilan, Y., et al. 2013, A\&A, 551, A131

Verner, D. A., Ferland, G. J., Korista, K. T., \& Yakovlev, D. G. 1996, ApJ, 465, 487

Vetter, R., Zülicke, L., Koch, A., van Dishoeck, E. F., \& Peyerimhoff, S. D. 1996, J. Chem. Phys., 104, 5558

Viala, Y. P., Letzelter, C., Eidelsberg, M., \& Rostas, F. 1988, A\&A, 193, 265

Visser, R., Geers, V. C., Dullemond, C. P., et al. 2007, A\&A, 466, 229

Visser, R., van Dishoeck, E. F., \& Black, J. H. 2009, A\&A, 503, 323

Wakelam, V., Herbst, E., Loison, J.-C., et al. 2012, ApJS, 199, 21

Wakelam, V., Smith, I. W. M., Herbst, E., et al. 2010, Space Sci. Rev., 156, 13

Walsh, C., Harada, N., Herbst, E., \& Millar, T. J. 2009, ApJ, 700, 752

Walsh, C., Millar, T. J., \& Nomura, H. 2010, ApJ, 722, 1607

Walsh, C., Millar, T. J., \& Nomura, H. 2013, ApJ, 766, L23

Walsh, C., Nomura, H., \& van Dishoeck, E. 2015, A\&A, 582, A88

Walter, C. W., Cosby, P. C., \& Helm, H. 1993, J. Chem. Phys., 99, 3553

Wang, L., Reutt, J., Lee, Y., \& Shirley, D. 1988, J. Electron Spectr. Relat. Phenom., 47, 167

Wang, J.-H., Hsu, Y.-T., \& Liu, K. 1997, J. Phys. Chem. A, 101, 6593

Wang, K., Stephens, J. A., \& McKoy, V. 1990, J. Chem. Phys., 93, 7874

Watanabe, K., Matsunaga, F. M., \& Sakai, H. 1967, Appl. Opt., 6, 391

Weingartner, J. C., \& Draine, B. T. 2001, ApJ, 548, 296

Weisheit, J. C. 1972, Phys. Rev. A, 5, 1621

Weiss, M. J., Lawrence, G. M., \& Young, R. A. 1970, J. Chem. Phys., 52, 2867

Werner, M. W. 1970, ApJ, 6, 81

West, G. A., \& Berry, M. J. 1974, J. Chem. Phys., 61, 4700

Wheeler, M. D., Newman, S. M., \& Orr-Ewing, A. J. 1998, J. Chem. Phys., 108, 6594

White, G. J. 1994, A\&A, 283, L25

Willitsch, S., Innocenti, F., Dyke, J. M., \& Merkt, F. 2005, J. Chem. Phys., 122, 024311

Willitsch, S., Merkt, F., Kállay, M., \& Gauss, J. 2006, Mol. Phys., 104, 1457

Wilson, S. H. S., Ashfold, M. N. R., \& Dixon, R. N. 1994, J. Chem. Phys., 101, 7538

Wishart, A. W. 1979, J. Phys. B, 12, 3511

Woitke, P., Kamp, I., \& Thi, W.-F. 2009, A\&A, 501, 383

Wolniewicz, L. 1995, J. Chem. Phys., 103, 1792

Woods, T. N., Prinz, D. K., Rottman, G. J., et al. 1996, J. Geophys. Res. - Atmos., 101,9541

Wu, C. Y. R., \& Judge, D. L. 1983, J. Chem. Phys., 78, 2180

Wu, C. Y. R., Chien, T. S., Liu, G. S., Judge, D. L., \& Caldwell, J. J. 1989, J. Chem. Phys., 91, 272

Wu, C. R., Yang, B., Chen, F., et al. 2000, Icarus, 145, 289

Wu, Y.-J., Lu, H.-C., Chen, H.-K., et al. 2007, J. Chem. Phys., 127,

Wu, Y.-J., Lin, M.-Y., Chou, S.-L., et al. 2010, ApJ, 721, 856

Xia, T., Chien, T., Wu, C., \& Judge, D. 1991, J. Quant. Spectr. Rad. Transf., 45, 77

Xing, W., Shi, D., Sun, J., Liu, H., \& Zhu, Z. 2013, Mol. Phys., 111, 673

Xu, H., \& Joens, J. A. 1993, Geophys. Res. Lett., 20, 1035

Yang, H., Herczeg, G. J., Linsky, J. L., et al. 2012, ApJ, 744, 121

Yih, T., Wu, H., Chu, C., et al. 1998, J. Korean. Phys. Soc., 32, 405 
Yoshino, K., Cheung, A.-C., Esmond, J., et al. 1988, Planet. Space Sci., 36, 1469 Yoshino, K., Esmond, J., Cheung, A.-C., Freeman, D., \& Parkinson, W. 1992, Planet. Space Sci., 40, 185

Yoshino, K., Esmond, J., Parkinson, W., Ito, K., \& Matsui, T. 1996, Chem. Phys., 211,387

Yoshino, K., Esmond, J., Parkinson, W., Ito, K., \& Matsui, T. 1997, Chem. Phys., 215, 429

Yoshino, K., Thorne, A. P., Murray, J. E., et al. 2006, J. Chem. Phys., 124, 054323

Yu, H. T., Sevin, A., Kassab, E., \& Evleth, E. M. 1984, J. Chem. Phys., 80, 2049

Zatsarinny, O., \& Bartschat, K. 2013, J. Phys. B, 46, 112001

Zatsarinny, O., \& Tayal, S. S. 2010, Phys. Rev. A, 81, 043423

Zeiri, Y., \& Balint-Kurti, G. 1983, J. Mol. Spectr., 99, 1

Zelikoff, M., \& Watanabe, K. 1953, J. Opt. Soc. Amer., 43, 756

Zhang, J., Imre, D. G., \& Frederick, J. H. 1989, J. Phys. Chem., 93, 1840

Zhao, Y., Cao, M., Li, Y., et al. 2014, J. Electron Spectr. Relat. Phenom., 196, 181, Advances in Vacuum Ultraviolet and X-ray Physics, 38th Int. Conf. Vacuum Ultraviolet and X-ray Physics (VUVX2013), University of Science and Technology of China

Zhen, J., Castellanos, P., Paardekooper, D. M., et al. 2015, ApJ, 804, L7

Zhen, J., Castillo, S. R., Joblin, C., et al. 2016, ApJ, 822, 113

Zhou, L., \& Xie, D. 2015, J. Phys. Chem. A, 119, 12062

\section{Appendix A: Shielding by atomic $\mathrm{H}$ and $\mathrm{C}$}

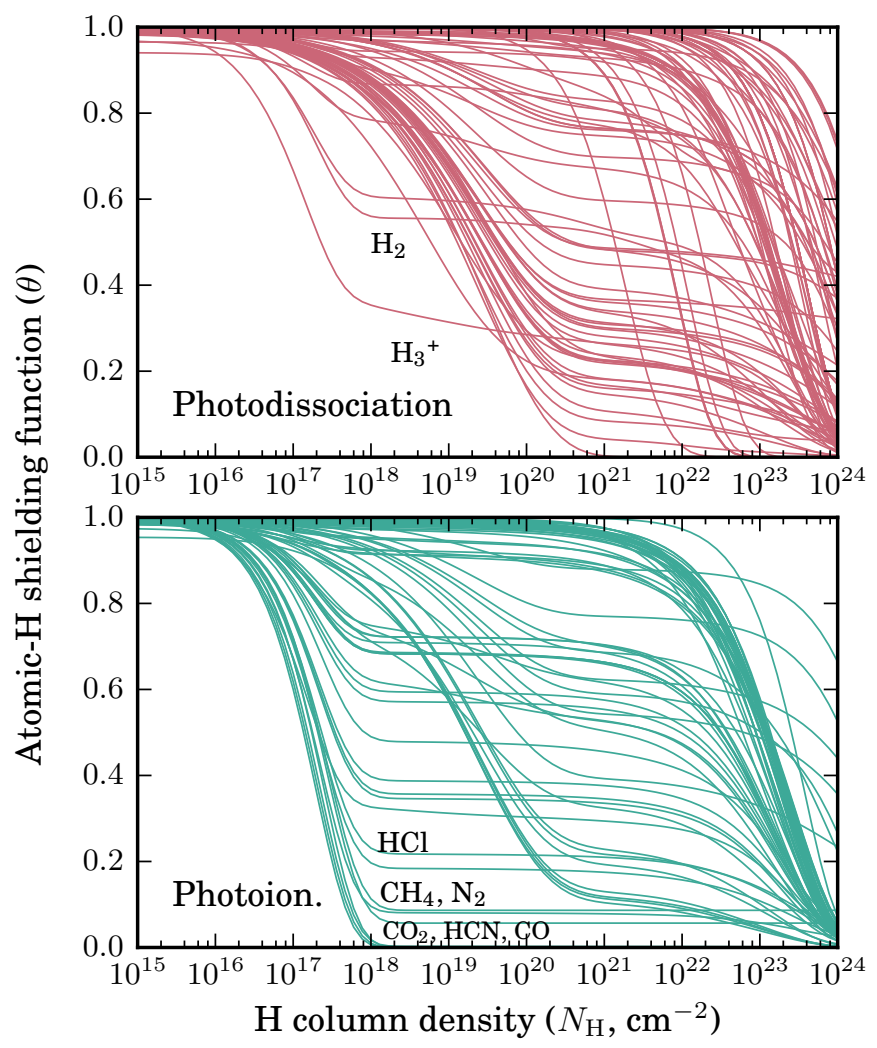

Fig. A.1. Shielding of photodissociation and photoionisation by atomic $\mathrm{H}$ in the simulated TW-Hydra radiation field.

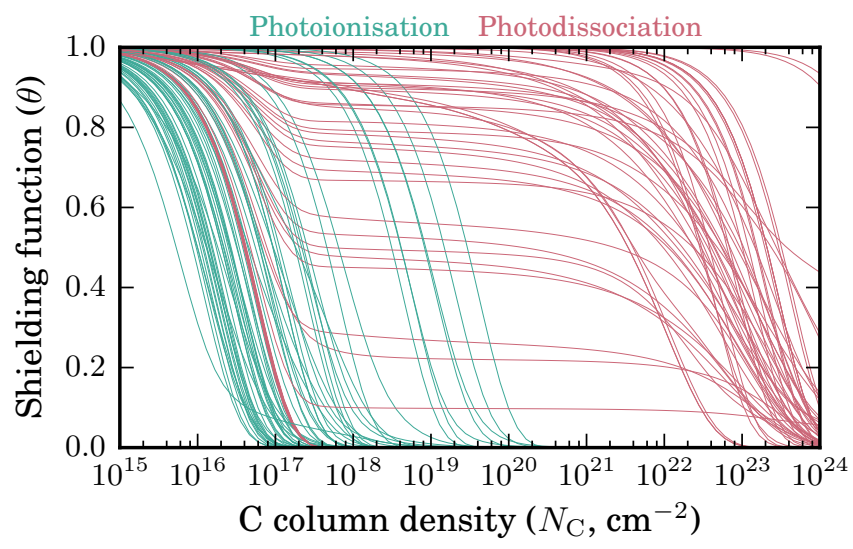

Fig. A.2. Shielding of photodissociation and ionisation in the ISRF by atomic $\mathrm{C}$.

Our simulated TW-Hydra radiation field, and other potential ultraviolet radiation environments, include shorter wavelengths than $91.2 \mathrm{~nm}$ and photoprocesses there may be sensitive to shielding by atomic $\mathrm{H}$. A collection of functions for $\mathrm{H}$-shielding in the TW-Hydra radiation field are shown in Fig. A.1. The photoionisation shielding by $\mathrm{H}$ for some molecular species (and photodissociation in the case of $\mathrm{H}_{3}^{+}$) is significant for $\mathrm{H}$ columns as low as $10^{17} \mathrm{~cm}^{-2}$.

Photoabsorption lines of $\mathrm{H}$ longwards of its ionisation limit were also included and contribute to the shielding of molecules that dissociate predominantly between $91.2 \mathrm{~nm}$ and the Lyman$\alpha$ wavelength, $121.6 \mathrm{~nm}$. Of the entire series of Lyman-lines converging on the $\mathrm{H}$ ionisation limit, some of which are shown in Fig. 25, the Lyman- $\alpha$ transition absorbs more photons than all others combined. It should be noted that the extinction of Lyman- $\alpha$ wavelength radiation is complicated by its re-radiation and forward scattering from dust grains (e.g., Bethell \& Bergin 2011).

The $110 \mathrm{~nm}$-threshold ionisation continuum of $\mathrm{C}$ will significantly reduce the photodissociation and ionisation rates of atomic and molecular species with cross sections biased to shorter wavelengths, assuming a column density of at least $10^{17} \mathrm{~cm}^{-2}$. Shielding functions for the ISRF are shown in Fig. A.2 as functions of C column density and clearly demonstrate this critical density. The requisite amount of $\mathrm{C}$ is observed and modelled to exist in some kinds of photodissociation regions (Werner 1970; Frerking et al. 1989; Hollenbach et al. 1991; Hasegawa \& Kwok 2003). For molecules with photodissociation cross sections predominantly longwards of $110 \mathrm{~nm}$ an extremely unlikely $\mathrm{C}$ column density of at least $10^{22} \mathrm{~cm}^{-2}$ is required for shielding. In this case, photoabsorption of nonionising photoabsorption into excited $\mathrm{C}$ levels eventually provides the necessary opacity. 